NUCLEAR ENERGY RESEARCH INITIATIVE PROGRAM (NERI)

U.S. Department of Energy NERI Project No. 2005-094

\title{
UTILIZATION OF MINOR ACTINIDES AS A FUEL COMPONENT FOR ULTRA-LONG LIFE VHTR CONFIGURATIONS: DESIGNS, ADVANTAGES AND LIMITATIONS
}

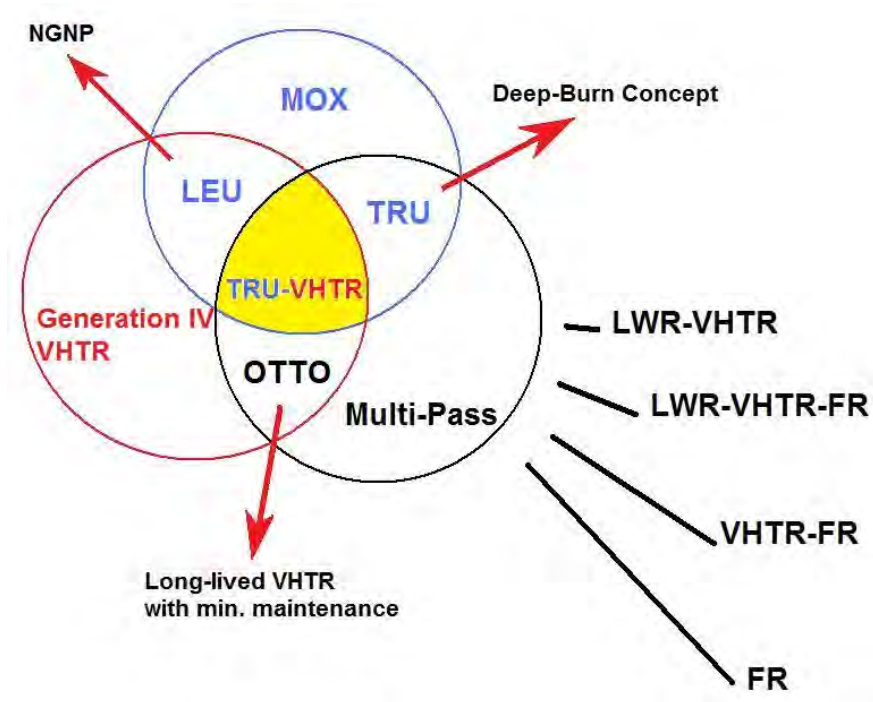

\section{FINAL \\ Scientific/Technical Report}

Dr. Pavel V. Tsvetkov

Department of Nuclear Engineering

Texas A\&M University

3133 TAMU, College Station, TX 77843-3133

Phone: (979) 845-4161, Fax: (979) 845-6443, E-mail: tsvetkov@tamu.edu 
NUCLEAR ENERGY RESEARCH INITIATIVE PROGRAM (NERI)

U.S. Department of Energy NERI Project No. 2005-094

\section{UTILIZATION OF MINOR ACTINIDES AS A FUEL COMPONENT FOR ULTRA-LONG LIFE VHTR CONFIGURATIONS: DESIGNS, ADVANTAGES AND LIMITATIONS}

March 14, 2005 - March 13, 2008

Award Number: DE-FC07-05ID14655

\section{FINAL \\ Scientific/Technical Report}

Texas Engineering Experiment Station (TEES)

Office of Sponsored Research

3000 TAMU, College Station, TX 77843-3000

Dr. Pavel V. Tsvetkov

Department of Nuclear Engineering

Texas A\&M University

3133 TAMU, College Station, TX 77843-3133

Phone: (979) 845-4161, Fax: (979) 845-6443, E-mail: tsvetkov@tamu.edu 
U.S. Department of Energy NERI Project No. 2005-094

Award Number: DE-FC07-05ID14655

\section{UTILIZATION OF MINOR ACTINIDES AS A FUEL COMPONENT FOR ULTRA-LONG LIFE VHTR CONFIGURATIONS: DESIGNS, ADVANTAGES AND LIMITATIONS}

March 14, 2005 - March 13, 2008

\section{FINAL Scientific/Technical Report}

Project Report, November 2008

\section{Recipient:}

Texas Engineering Experiment Station (TEES), Office of Sponsored Research 3000 TAMU, College Station, TX 77843-3000

\section{Project Director (Principal Investigator):}

Dr. Pavel V. Tsvetkov

Phone: (979) 845-4161, Fax: (979) 845-6443, E-mail: tsvetkov@tamu.edu

\section{Contributors:}

Celestino Abrego

Ayodeji B. Alajo

David E. Ames II

Avery Guild-Bingham

Tuan Huang

Tom Lewis

Brian Massingill

Michael Naramore

Megan L. Pritchard

Timothy Rogers

Christopher Van der Hoeven

Ni Zhen

Department of Nuclear Engineering, Texas A\&M University

3133 TAMU, College Station, TX 77843-3133 
Utilization of MAs as a Fuel Component for Ultra-Long Life

VHTR Configurations: Designs, Advantages and Limitations

Project 05-094

Final Scientific/Technical Report

\section{DISCLAIMER OF WARRANTIES AND LIMITATION OF LIABILITIES}

This report was prepared by the organization(s) named below as an account of work sponsored by the Department of Energy (DOE) of the United States Government. Neither DOE, the United States Government, its sub-contractors, the organization(s) below, nor any agency thereof, nor any of their employees, nor any person acting on behalf of any of them:

(A) Makes any warranty, express or implied, or assumes any legal liability or responsibility for the accuracy, completeness, or usefulness of any information, apparatus, product, or process disclosed, or represents that its use would not infringe privately owned rights.

(B) Reference herein to any specific commercial product, process, or service by trade name, trademark, manufacturer, or otherwise does not necessarily constitute or imply its endorsement, recommendation, or favoring by the United States Government or any agency thereof.

The views and opinions of authors expressed herein do not necessarily state or reflect those of the United States Government or any agency thereof.

\section{Organization (s) that prepared this document:}

Department of Nuclear Engineering

Texas A\&M University

3133 TAMU, College Station, TX 77843-3133

Texas Engineering Experiment Station (TEES)

Office of Sponsored Research

3000 TAMU, College Station, TX 77843-3000

This is a final scientific/technical report to the Department of Energy for the Nuclear Energy Research Initiative Program (NERI) work. 
Utilization of MAs as a Fuel Component for Ultra-Long Life

VHTR Configurations: Designs, Advantages and Limitations

Project 05-094

Final Scientific/Technical Report

\section{CITATIONS AND ACKNOWLEDGMENT OF FEDERAL SUPPORT}

This document was prepared by:

Department of Nuclear Engineering

Texas A\&M University

3133 TAMU,

College Station, TX 77843-3133

Texas Engineering Experiment Station (TEES)

Office of Sponsored Research

3000 TAMU, College Station, TX 77843-3000

Principal Investigator:

Dr. Pavel V. Tsvetkov

Phone: (979) 845-4161

Fax: (979) 845-6443

E-mail: tsvetkov@tamu.edu

This document is based upon work supported by the Department of Energy under Award Number DE-FC07-05ID14655 (05-094). 


\section{ABSTRACT}

This project assessed the advantages and limitations of using minor actinides as a fuel component to achieve ultra-long life Very High Temperature Reactor (VHTR) configurations. Researchers considered and compared the capabilities of pebble-bed and prismatic core designs with advanced actinide fuels to achieve ultra-long operation without refueling. Since both core designs permit flexibility in component configuration, fuel utilization, and fuel management, it is possible to improve fissile properties of minor actinides by neutron spectrum shifting through configuration adjustments. The project studied advanced actinide fuels, which could reduce the long-term radio-toxicity and heat load of high-level waste sent to a geologic repository and enable recovery of the energy contained in spent fuel. The ultra-long core life autonomous approach may reduce the technical need for additional repositories and is capable to improve marketability of the Generation IV VHTR by allowing worldwide deployment, including remote regions and regions with limited industrial resources. Utilization of minor actinides in nuclear reactors facilitates developments of new fuel cycles towards sustainable nuclear energy scenarios. 


\section{CONTENTS}

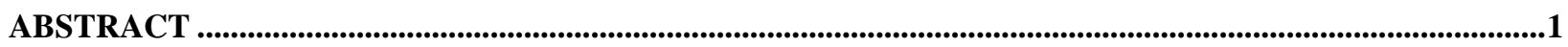

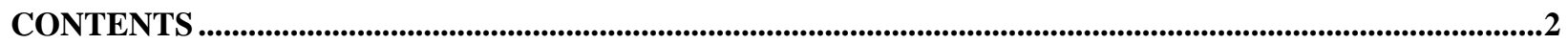

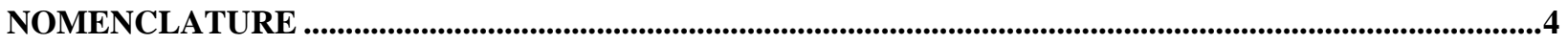

1. EXECUTIVE SUMMARY ..............................................................................................................................

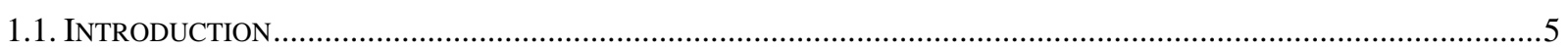

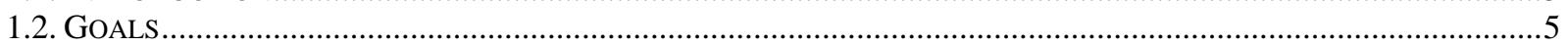

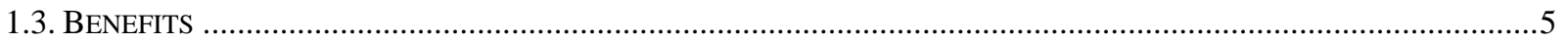

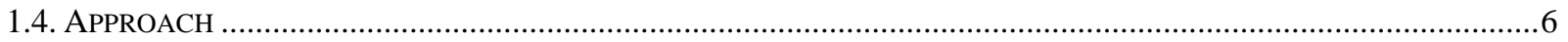

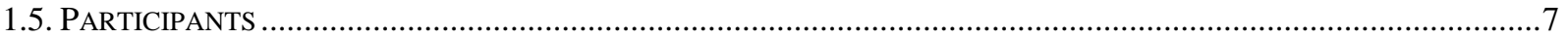

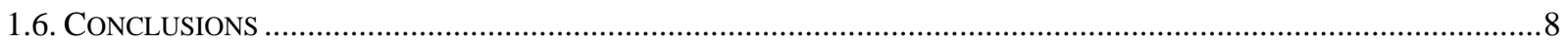

2. HIGH FIDELITY 3D WHOLE-CORE EXACT GEOMETRY MODELING APPROACH.............................10

3. BENCHMARK EFFORTS TO SUPPORT STUDIES OF ADVANCED VHTRS ...........................................18

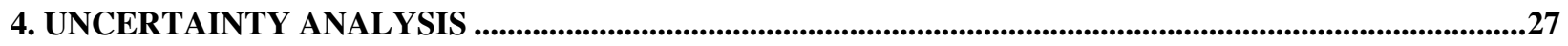

4.1. UNCERTAINTY EFFECTS DUE TO NUCLEAR DATA ……………...................................................................

4.2 SENSITIVITY OF THE VHTR CORE PERFORMANCE tO tHE NEUTRON-KERNEL INTERACTIONS ...........................54

5. CONFIGURATION ADJUSTMENT POTENTIAL OF VHTRS WITH ACTINIDE FUELS.........................56

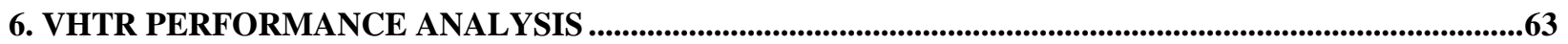

6.1 UTILIZATION OF TRUS AS A FUEL FOR VHTRs: COMPOSITIONS, NEUTRONICS IMPACT AND SAFETY ................73

6.2. ANALYSIS OF TRU-FUELED VHTR PRISMATIC CORE PERFORMANCE DOMAINS...............................................75

6.3. TRU-FuELED VHTRs: DESIGN, PERFORMANCE AND APPLICATIONS ........................................................77

6.4. TRU-FuELED VHTRS FOR APPLICATIONS REQUIRING AN EXTENDED OPERATION WITH MINIMIZED CONTROL

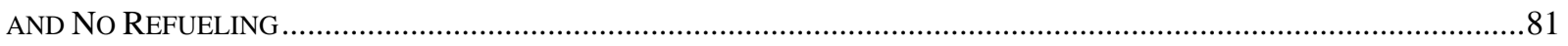

6.5. OUT-OF-CORE FUEL CYCLE CHARACTERISTICS OF VHTRS WITH NO ON-SITE REFUELING ..............................89

6.6. UTILIZATION OF TRUs in VHTRs - OpERATION IN A Single-BATCH MOdE: Front END, BACK END, AND

PERFORMANCE ..........................................................................................................................................

6.7. REACTOR Physics of VHTRs WITH $\mathrm{UO}_{2}$, UCO \& $\mathrm{UC}_{0.5} \mathrm{O}_{1.5}$ KERNELS OpERATING WITHOUT ONSITE

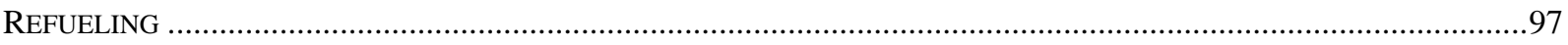

6.8. CORE LIFETIME AND FUEL UTILIZATION IN PRISMATIC VHTR CORES .....................................................99

6.9. VHTR-BASED SYSTEMS FOR AUTONOMOUS CO-GENERATION APPLICATIONS ...............................................105

6.10. Autonomous Control StRATEgIES FOR VHTR-BASEd SySTEMS FOR HydRogen PRODUCTION ..............114

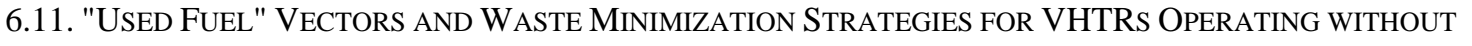

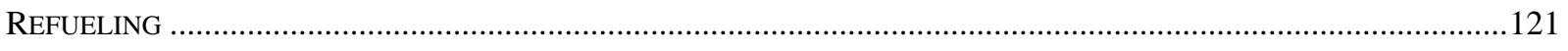

7. CONCLUSIONS AND FOLLOW-ON .............................................................................................................131

$\begin{array}{lll}\text { Department of Nuclear Engineering } & 2 & \text { Texas A\&M University }\end{array}$

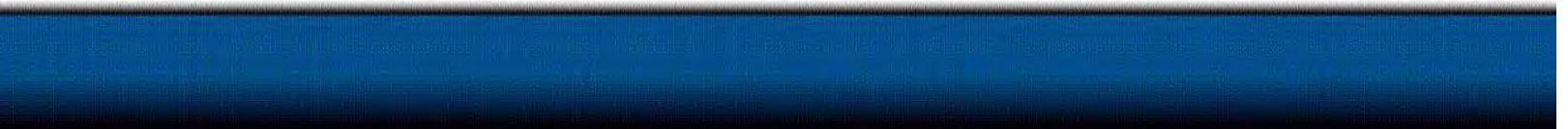


Utilization of MAs as a Fuel Component for Ultra-Long Life VHTR Configurations: Designs, Advantages and Limitations

Project 05-094

Final Scientific/Technical Report

8. BIBLIOGRAPHY 134

9. APPENDIX A. PROJECT PUBLICATIONS ...................................................................................................151

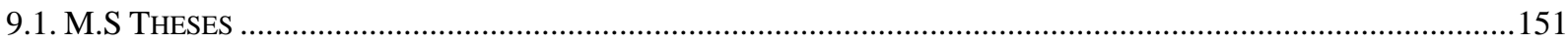

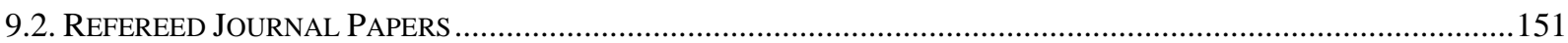

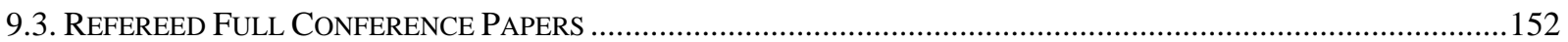

9.4. REFEREED CONFERENCE SUMMARIES (ANS TRANSACTIONS) ...............................................................153

10. APPENDIX B. PROTOTYPE SPECIFICATIONS OF SMALL-SCALE VHTRS......................................155

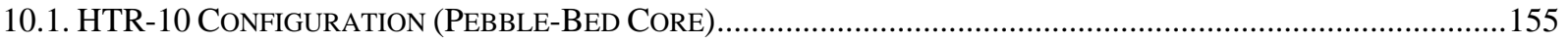

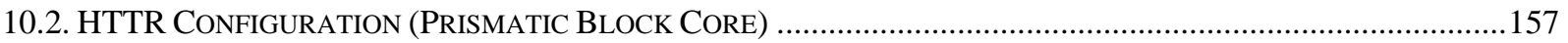

12.3 DOCUMENTED BENCHMARK PROBLEMS FOR HTTR AND HTR-10 CONFIGURATIONS ................................164

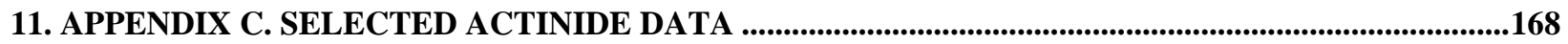

12. APPENDIX D. PROJECT PARTICIPANTS AND RESOURCES......................................................171

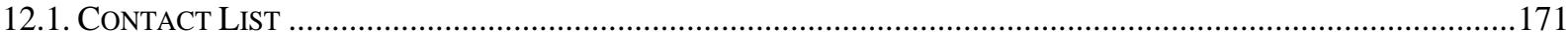

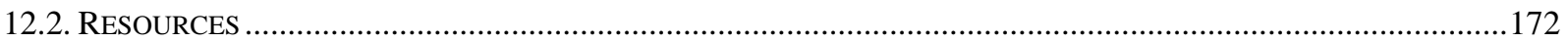

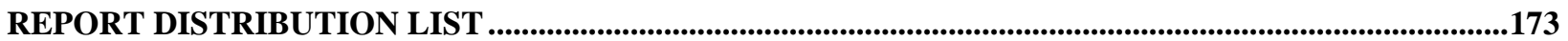


Utilization of MAs as a Fuel Component for Ultra-Long Life VHTR Configurations: Designs, Advantages and Limitations

\section{NOMENCLATURE}

$\begin{array}{ll}\text { AFCI } & \text { - Advanced Fuel Cycle Initiative } \\ \text { BCC } & \text { - Body-Centered Cubic lattice } \\ \text { BOL } & \text { - Beginning-Of-Life } \\ \text { C/HM } & \text { - Carbon-to-Heavy Metal Atom Ratio } \\ \text { DB } & \text { - Deep-Burn concept } \\ \text { DU } & \text { - Depleted Uranium } \\ \text { EOL } & \text { - End-Of-Life } \\ \text { FP } & \text { - Fission Products } \\ \text { HEU } & \text { - Highly Enriched Uranium } \\ \text { HLW } & \text { - High Level Wastes } \\ \text { HTGR } & \text { - High Temperature Gas-cooled Reactor } \\ \text { HTTR } & \text { - High Temperature Test Reactor } \\ \text { IMF } & \text { - Inert Matrix Fuel } \\ \text { JAERI } & \text { - Japan Atomic Energy Research Institute } \\ \text { LANL } & \text { - Los Alamos National Laboratory } \\ \text { LEU } & \text { - Low-Enriched Uranium } \\ \text { LWR } & \text { - Light Water Reactor } \\ \text { MA } & \text { - Minor Actinides } \\ \text { MO } & \text { - Multi-objective Optimization } \\ \text { MOP } & \text { - Multi-objective Optimization Problem } \\ \text { MOX } & \text { - Mixed Oxide } \\ \text { MTHM } & \text { - Metric Tons of Heavy Metal } \\ \text { NERI } & \text { - Nuclear Energy Research Initiative } \\ \text { NGNP } & \text { - Next Generation Nuclear Plant } \\ \text { ORNL } & \text { - Oak Ridge National Laboratory } \\ \text { OTTO } & \text { - Once-Through-Then-Out } \\ \text { P\&T } & \text { - Partitioning and Transmutation } \\ \text { PyC } & \text { - Pyrolytic Carbon } \\ \text { PWR } & \text { - Pressurized Water Reactor } \\ \text { RGPu } & \text { - Reactor Grade Plutonium } \\ \text { SNF } & \text { - Spent Nuclear Fuel } \\ \text { TF } & \text { - Transmutation Fuel } \\ \text { TRU } & \text { - Transuranium Nuclides } \\ \text { VHTR } & \text { - Very High Temperature Reactor } \\ \text { YSZ } & \text { - Yttria Stabilized Zirconia } \\ & \end{array}$




\section{EXECUTIVE SUMMARY}

\subsection{Introduction}

Partitioning and transmutation of minor actinides are expected to have a positive impact on the future of nuclear technology. Their deployment would lead to incineration of hazardous nuclides and could potentially provide additional fuel supply. The project assesses the possibility, advantages and limitations of involving minor actinides as a fuel component. The analysis takes into consideration and compares capabilities of actinide-fueled VHTRs with pebble-bed and prismatic cores to approach a reactor lifetime long operation without intermediate refueling.

A hybrid Monte Carlo-deterministic methodology has been adopted for coupled neutronicsthermal hydraulics design studies of VHTRs. Within the computational scheme, the key technical issues are being addressed and resolved by implementing efficient automated modeling procedures and sequences, combining Monte Carlo and deterministic approaches, developing and applying realistic 3D coupled neutronics-thermal-hydraulics models with multiheterogeneity treatments, developing and performing experimental/computational benchmarks for model verification and validation, analyzing uncertainty effects and error propagation.

This report describes the modeling approach, discusses benchmark results and the analysis of actinide-fueled VHTRs.

\subsection{Goals}

The goal of this project is to assess the possibility, advantages and limitations of achieving ultra-long life VHTR (Very High Temperature Reactor) configurations by utilizing minor actinides (MAs) as a fuel component. The analysis takes into consideration and compares capabilities of pebble-bed and prismatic core designs with advanced actinide fuels to approach the reactor lifetime long operation without intermediate refueling.

\subsection{Benefits}

The project investigates VHTRs with advanced actinide fuels, which could reduce the longterm radiotoxicity and heat load of high-level waste sent to a geologic repository and enable recovery of the energy contained in spent fuel. The ultra-long core life approach reduces the technical need for additional repositories per decade of reactor operation and should improve 
marketability of the Generation IV VHTR by allowing worldwide deployment including in developing countries. Utilization of MAs facilitates development of new fuel cycles and supports fuel supply sustainability. Thus, this research comprehensively addresses both intermediate and long-term issues associated with high-level nuclear wastes and should demonstrate the potential of VHTR-based solutions.

\subsection{Approach}

As supported by the studies within the project framework, the principal mechanism to achieve ultra-long life VHTR configurations is an enhanced involvement of self-generated fissile compositions based on a spent LWR fuel. Since pebble bed and prismatic core designs permit flexibility in component configuration, fuel utilization and management, it is possible to improve fissile properties of MAs by neutron spectrum shifting through configuration adjustments.

The ultra-long life VHTR systems are developed and analyzed focusing on control, dynamics, safety, and proliferation-resistance during reactor lifetime long autonomous operation. The developed coupling methodology and tools account for multi-heterogeneity of the VHTR configurations and allow whole-core/system 3D modeling of VHTRs.

To assure comprehensive, realistic assessment of the VHTR design and operation targeting passive safety confirmation, the adequacy of computational methods and models used to compute performance characteristics is supported by comparisons with experimental data covering an appropriate range of conditions. The VHTR model benchmark sets were compiled based on the LEU-HTR PROTEUS, HTTR, and HTR-10 experimental programs. Validation and verification of the VHTR models and the preliminary studies of the VHTRs with MAs are completed.

In addition to the experimental benchmark confirmation of the developed models and obtained results, the success of the project is determined by its ability to demonstrate possibility (or prove impractical), advantages (or show clear disadvantages), and limitations of achieving ultra-long life VHTR configurations by utilizing MAs as a fuel component. The results are in agreement with the available data and confirm the chosen approach. Studies of VHTRs with MAs suggest promising performance.

To support analysis of the VHTR configurations with advanced actinide fuels, extensive literature review studies were conducted. The performed worldwide literature survey is the basis of the developed actinide fuels and HTGR materials properties database. This database is used for realistic VHTR configurations with advanced materials. The code systems and tools for evaluations of uncertainty effects (nuclear and design/performance uncertainties) were developed 


\section{Utilization of MAs as a Fuel Component for Ultra-Long Life} VHTR Configurations: Designs, Advantages and Limitations

Project 05-094

Final Scientific/Technical Report

on the basis of existing software packages to support developed uncertainty analysis methodologies.

\subsection{Participants}

Over the duration of the project, 14 students at all levels (undergraduate, graduate M.S. and Ph.D.) participated in the studies and contributed their ideas, results, findings, and observations to this report. Table 1 provides the list of students who were major contributors. Each student represents specific areas of the project.

Table 1. Leading Project Participants

\begin{tabular}{|c|c|c|}
\hline Name & Position & Area of the Project \\
\hline \multirow[t]{5}{*}{ Ayodeji B. Alajo } & \multirow[t]{5}{*}{ Graduate } & Advanced Actinide Fuels \\
\hline & & VHTRs with MAs \\
\hline & & Uncertainty Due to Nuclear Data \\
\hline & & LWR Compositions and their Fluctuations \\
\hline & & LWR Spent Fuel in TRU-Fueled VHTRs \\
\hline \multirow[t]{5}{*}{ David E. Ames II } & \multirow[t]{5}{*}{ Graduate } & Core/System 3D Models \\
\hline & & V\&V VHTR Prismatic/Pebble- Bed Models \\
\hline & & HTTR-to-VHTR Design Development \\
\hline & & Uncertainty Due to Parameters \\
\hline & & VHTR Configurations with MAs \\
\hline \multirow[t]{2}{*}{ Avery Guild-Bingham } & \multirow[t]{2}{*}{ Graduate } & Advanced Actinide Fuels \\
\hline & & Monte Carlo modeling \\
\hline \multirow[t]{3}{*}{ Tom Lewis } & \multirow[t]{3}{*}{ Graduate } & Advanced Actinide Fuels \\
\hline & & Coupling Methodology and Tools \\
\hline & & $\begin{array}{l}\text { Analysis of TRU-Fueled VHTR Prismatic Core Performance } \\
\text { Domains }\end{array}$ \\
\hline \multirow[t]{3}{*}{ Megan L. Pritchard } & \multirow[t]{3}{*}{ Undergraduate } & V\&V VHTR Pebble-Bed Core Model \\
\hline & & Uncertainty Due to Parameters \\
\hline & & Neutronics Analysis of Pebble Bed Cores with TRUs \\
\hline \multirow[t]{2}{*}{ Timothy J. Rogers } & \multirow[t]{2}{*}{ Undergraduate } & Computer Support \\
\hline & & Website Support \\
\hline Ni Zhen & Undergraduate & VHTR Materials \\
\hline
\end{tabular}




\subsection{Conclusions}

Although indicating some technical limitations and challenges, studies of VHTRs with TRUs/MAs definitely suggest promising performance and possibility to utilize the core configurations with TRUs/MAs gaining prolonged operation and self-sustainability:

- There are significant differences between LEU- and TRUs-fueled VHTRs. These differences affect system performance characteristics during operation.

- A single batch-mode operation appears to be potentially possible. Core lifetimes up to 7.5 years have been observed in the present analysis.

- Fluence-related limitations (radiation damage) are the most significant constraints on achievable operation times. This constraint can be relaxed or even eliminated by reducing power density levels and using advanced radiation-tolerant materials for extended-life VHTR configurations. However, use of advanced materials will adversely impact economics characteristics.

- Acceptable safety characteristics have been observed for all configurations. However, low delayed neutron yields may result in design challenges.

Thus, the TRU-fueled VHTRs offer performance characteristics that would be difficult to achieve in analogous LEU-fueled systems - almost a decade-long batch mode operation without intermediate refueling, significant reductions of initial excess reactivity levels (smaller lifetime reactivity swings), and inherently higher achievable burnup levels.

At the same time, the results clearly illustrate that use of TRU vectors as a fuel inherently facilitates development of specially designed VHTRs with core materials withstanding performance conditions of systems optimized for fuel loading with TRUs. Limiting fast fluences lead to larger resulting radiation damage effects. There are significant core physics differences due to spectral shift effects towards harder neutron spectra with substantially reduced neutron populations at thermal energies.

The research work advanced according to the 3-years plan and has been completed in full. No scope-related changes were made from the DOE approved application. The project accomplishments are fully consistent with the project goals/objectives. Table 2 summarizes the milestones associated with the project work scope. The project results have been extensively reported at the ANS and ASME conferences as well as have been published in archival peerreviewed journals. 
Utilization of MAs as a Fuel Component for Ultra-Long Life VHTR Configurations: Designs, Advantages and Limitations

\section{Table 2. Milestones}

0. Research Kickoff and Planning

0.1. Software Survey/Upgrade

0.2. Announcement and Final Selection of Students

1. Development of Core/System 3D Models with Explicit Multi-Heterogeneity Treatments

1.1. VHTR Technology Literature Review

1.2. Explicit Multi-Heterogeneity Modeling

1.3. Coupling Methodology and Tools

1.4. Whole-Core/System 3D Models of VHTRs

2. Development of Benchmark Problems to Compare with Experimental Data

2.1. HTTR, HTR-10, PROTEUS Benchmarks

2.2. Compiled VHTR Model Benchmark Sets

\section{Validation \& Verification of the VHTR Model}

4. Analysis of Uncertainty Effects on VHTR Performance Characteristics

4.1. Advanced Actinide Fuels Literature Review

4.2. Actinide Fuels Properties Database

4.3. Preliminary Analysis of VHTRs with MAs

4.4. Uncertainty Analysis Methodology and Tools

4.5. Uncertainty Effects due to Nuclear Data

4.6. Uncertainty Effects due to VHTR Parameters

4.7. Reliability Analysis of the VHTR Modeling

5. Analysis of Configuration Variation Capabilities to Achieve Ultra-Long Operation without Refueling, Maximize Burn-Up and Minimize Reactivity Swings

5.1. Variation Analysis Methodology and Tools

5.2. Analysis of VHTR Geometry Variations

5.3. Analysis of VHTR Materials Variations

5.4. VHTR Optimization to Achieve Ultra-Long Life

5.5. Feasibility of Ultra-Long Life VHTRs with MAs

5.6. Ultra-Long Life VHTRs and Transmutation

6. Control, Dynamics, Safety, and Proliferation-Resistance Studies of Ultra-Long Life VHTRs with Advanced Actinide Fuels

6.1. Dynamics, Safety and Control of VHTRs with MAs

6.2. Fuel Cycle of Ultra-Long Life VHTRs with MAs

6.3. Proliferation-Resistance of Ultra-Long Life VHTRs

\section{Final Report}




\section{High Fidelity 3D Whole-Core Exact Geometry Modeling Approach}

Partitioning and transmutation of minor actinides are expected to have a positive impact on the future of nuclear technology. Their deployment would lead to incineration of hazardous nuclides and could potentially provide additional fuel supply. The U.S. DOE NERI Project assesses the possibility, advantages and limitations of involving minor actinides as a fuel component. The analysis takes into consideration and compares capabilities of actinide-fueled VHTRs with pebble-bed and prismatic cores to approach a reactor lifetime long operation without intermediate refueling. A hybrid Monte Carlo-deterministic methodology has been adopted for coupled neutronics-thermal hydraulics design studies of VHTRs. Within the computational scheme, the key technical issues are being addressed and resolved by implementing efficient automated modeling procedures and sequences, combining Monte Carlo and deterministic approaches, developing and applying realistic 3D coupled neutronics-thermalhydraulics models with multi-heterogeneity treatments, developing and performing experimental/computational benchmarks for model verification and validation, analyzing uncertainty effects and error propagation. This paper introduces the suggested modeling approach, discusses benchmark results and the preliminary analysis of actinide-fueled VHTRs. The presented up-to-date results are in agreement with the available experimental data. Studies of VHTRs with minor actinides suggest promising performance. 


\title{
Coupled Hybrid Monte Carlo - Deterministic Analysis of VHTR Configurations with Advanced Actinide Fuels
}

\author{
Pavel V. Tsvetkov. David E. Ames II, Ayodeji B. Alajo and Megan L. Pritchard \\ Department of Nuclear Engineering, Texas A\&M University \\ 129 Zachry Engineering Center, MS 3133 TAMU, College Station, TX, 77843-3133 \\ Tel: (979) 845-7078, Fax: (979) 845-6443, Email: Tsvetkov@tamu.edu
}

\begin{abstract}
Partitioning and transmutation of minor actinides are expected to have a positive impact on the future of nuclear technology. Their deployment would lead to incineration of hazardous nuclides and could potentially provide additional fuel supply. The U.S. DOE NERI Project assesses the possibility, advantages and limitations of involving minor actinides as a fuel component. The analysis takes into consideration and compares capabilities of actinide-fireled VHTRs with pebble-bed and prismatic cores to approach a reactor lifetime long operation without intermediate refueling. A hybrid Monte Carlo-deterministic methodology has been adopted for coupled neutronics-thermal hydraulics design studies of VHTRs. Within the computational scheme, the key technical issues are being addressed and resolved by implementing efficient automated modeling procedures and sequences, combining Monte Carlo and deterministic approaches, developing and applying realistic $3 D$ coupled neutronics-thermal-hydraulics models with multi-heterogeneity treatments, developing and performing experimental/computational benchmarks for model verification and validation, analyzing uncertainty effects and error propagation. This paper introduces the suggested modeling approach, discusses benchmark results and the preliminary analysis of actinide-fueled VHTRs. The presented up-to-date results are in agreement with the available experimental data. Studies of VHTRs with minor actinides suggest promising performance.
\end{abstract}

\section{INTRODUCTION}

Conventional fuel reprocessing technologies manage recovered minor actinides (MA) as high level wastes (HLW). Successful deployment of partitioning and transmutation (P\&T) of MAs would lead to incineration of these hazardous nuclides and could potentially provide additional fuel supply. ${ }^{1}$

The U.S. DOE NERI Project assesses the possibility, advantages and limitations of involving MAs as a fuel component. The analysis takes into consideration and compares capabilities of actinide-fueled VHTRs (Very High Temperature Reactors) with pebble-bed and prismatic cores to approach a reactor lifetime long operation without intermediate refueling.

The ultra-long life VHTR systems with MAs are developed and analyzed accounting for controllability, dynamics and safety margins, and proliferation-resistance during the entire reactor lifetime. The resulting VHTR configurations should have inherent capabilities for utilization of MAs from spent LWR fuel, reduction of spent fuel flows and handling per unit of produced energy, and potential for autonomous operation with minimized maintenance. Their broad deployment would allow reducing the long-term radiotoxicity and heat load of HLW sent to a geologic repository and enable recovery of the energy contained in spent fuel

Thus, the project evaluates potential linkage and application for Generation IV VHTRs in the AFCI (Advanced Fuel Cycle Initiative) Program. ${ }^{1,2}$ Utilization of MAs as a fuel component would facilitate development of new fuel cycles and support sustainability of a fuel source for nuclear energy assuring future operation of Generation IV nuclear energy systems. The ultra-long core life approach reduces the technical need for additional repositories per decade of reactor operation. Furthermore, the developed VHTR configurations should improve marketability of the Generation IV VHTRs intended for hydrogen production because their implementation allows worldwide deployment including developing countries.

Behavior of the advanced nuclear energy systems like Generation IV VHTRs is difficult to predict due to inherent 


\section{Utilization of MAs as a Fuel Component for Ultra-Long Life} VHTR Configurations: Designs, Advantages and Limitations

\section{Project 05-094}

\section{Final Scientific/Technical Report}

safety features resulting in tight coupling between energy production, transport and conversion, and system behavior during normal operation and off-normal situations. To create advanced nuclear energy systems it is desirable to have a modeling-based design development that relies on simulating features of the entire life cycle of the system before actual physical prototyping - from concept development to detailed design, prototyping, and safety analysis. This approach should provide consistency between neutronics and other performance aspects. The suggested integrated/hybrid Monte-Carlo-deterministic modeling approach offers the desirable analysis capabilities for the VHTR design studies. It was originally developed and successfully used for fission fragment magnetic collimator reactors. ${ }^{3}$

The use of Monte Carlo codes creates a potential for explicit 3D whole-core/reactor studies of the VHTR configurations addressing the key reactor physics areas such as the double and multi-level heterogeneity, neutron streaming in the low-density regions, and neutron spectrum transitions at the interfaces. Coupling approach is important for modeling of the dynamic behavior and assessment of inherent safety. Space and burnup dependent reactivity coefficients and feedback analysis have paramount importance for inherent safety confirmation.

\section{MULTI-HETEROGENEITY OF THE VHTR CORE}

In both design concepts, prismatic block and pebble bed, under consideration, the use of coated micro-particles creates double-heterogeneity that defines performance characteristics of the VHTR core. The first level of heterogeneity (micro-level) is formed by coated fuel micro-particles that are randomly distributed in the fuel region of the graphite fuel pebble (pebble-bed core) or in the graphite compact (prismatic graphite block core). The second level of heterogeneity (macro-level) is formed by the randomly-packed bed of pebbles or by the prismatic blocks with compacts that compose the VHTR core, respectively. For desirable performance characteristics, the pebble-bed core may also contain some number of dummy moderator pebbles to achieve the required moderator-tofuel ratio. The prismatic graphite block core has channels for coolant, instrumentation and handling operations. As a result, there are various neutron streaming passages formed by pebble-bed voids, coolant channels, instrumentation and handling channels, plenums, and etc. The proximity to outer and central reflector regions is also an important factor that influences the VHTR physics. Depending on a specific computational model, these features act and can be taken into account as multiple additional levels of heterogeneity up to the $3 \mathrm{D}$ finite core model that provides an appropriate comprehensive multi-heterogeneity treatment.
The resonance self-shielding effects are determined by the microscopic flux behavior in the fuel, which strongly depends on the geometry of the fuel configuration. As a result, the resonance absorption should be calculated on the basis of the microscopic lattice of coated fuel microparticles. The neutron mean free path in this lattice is so large compared to the dimensions of a pebble or a compact that it cannot be considered infinite. Therefore, other pebbles (pebble-bed core) or compacts (prismatic graphite block core) as well as the core features cannot be neglected. ${ }^{4,5}$

In the present study, recognizing reactor physics similarities of the prismatic block and pebble bed cores, the LEU-HTR PROTEUS pebble-bed configurations (LEUPRO 1 through 6) have been used to evaluate doubleheterogeneity effects at the lattice level and confirm their significance for proper modeling of the VHTR cores. ${ }^{6,7}$ The LEU-HTR PROTEUS benchmark specifications were chosen as an example due to their unique level of available technical details assuring realistic modeling as well as due to the availability of the LEU-HTR PROTEUS International Benchmark Program results.

TABLE I

Accounting for Double-Heterogeneity

(LEU-HTR PROTEUS Cores 1 - 6, Cumulative Analysis)

\begin{tabular}{|c|c|}
\hline Reaction Rate Ratio & Relative Effect (\%)* \\
\hline Epithermal-to-Thermal ${ }^{238} \mathrm{U}$ Capture & $-(30-50)$ \\
\hline Epithermal-to-Thermal ${ }^{235} \mathrm{U}$ Fission & $-(9-11)$ \\
\hline${ }^{238} \mathrm{U}$ Capture-to- ${ }^{235} \mathrm{U}$ Fission & $-(30-42)$ \\
\hline
\end{tabular}

*Effects of the exact modeling are computed relative to the simplified single-level macro-heterogeneity modeling.

Table I illustrates the double-heterogeneity effects and their significance. The provided relative effect ranges represent the summary over the LEUPRO cores 1 through 6. For each core configuration, the exact Monte Carlo calculations with MCNP were compared to the simplified single-level macro-heterogeneity approach. ${ }^{8}$ The ranges have been determined by selecting minimum and maximum contributions. For the illustration in this paper, Table I provides only the overall comparison for several spectral characteristics and does not detailize effects for each specific LEUPRO case.

It is apparent that the double-heterogeneity effects should be expected to play a defining role for all nuclides in the VHTR cores, especially those with resonance cross sections, including fuel nuclides like ${ }^{235} \mathrm{U}$ and ${ }^{238} \mathrm{U}$ and burnable absorbers like erbium. Furthermore, as compared to the exact geometry modeling using Monte Carlo codes, the simplified single-level macro-heterogeneity modeling could significantly over estimate fuel lifetimes in VHTRs.

III. MODELING APPROACH AND METHODOLOGY 


\section{Utilization of MAs as a Fuel Component for Ultra-Long Life} VHTR Configurations: Designs, Advantages and Limitations

Several technical challenges are associated with the analysis and development of ultra-long life VHTR configurations with MAs as a fuel component:

- whole core/system modeling with multiheterogeneity treatments,

- model benchmarking, uncertainty effects of nuclear data and design parameters including temperature dependence,

- error propagation during depletion calculations,

- large computational times affecting ability to consider many design configurations.

The computational scheme of the analysis of actinidefueled VHTRs is shown in Fig. 1. The VHTR modeling is based on and is supported by the following subject-specific external computer code systems:

- MCNP (3D Monte Carlo neutron transport calculations including multiplying systems);

in the present analysis, as described above, MCNP is used only for the lattice-level benchmark calculations of the LEU-HTR PROTEUS cores:

- SCALE (reactivity, criticality and fuel depletion calculations) $;^{9}$

in the present analysis, computational sequences of the SCALE code system are used for reactor physics calculations including static criticality and fuel depletion dynamics over the irradiation time;

- HeatWave (3D steady-state and dynamic thermal transport calculations with temperature-dependent materials $){ }^{10}$

in the present analysis, HeatWave is used to produce fine-scale temperature distributions at the whole core level up to temperature reconstructions in the fuel kernels;

- Several auxiliary computer codes for specialized data processing and modeling including calculations of Dancoff correction factors (Dancoff-MC, DENT 97, etc.) $)^{11,12}$

in the present analysis, Dancoff-MC and its modifications are used to generate Dancoff correction factors for the considered VHTR configurations; DENT 97 provides coupling interfaces between HeatWave and SCALE;

- General-purpose software packages and computer code systems for applied mathematics and graphics (MatLab, MathCAD, CAD systems, and etc.).

The scheme is followed as an overall design procedure for the VHTR system up to a power unit level. Fig. 1 emphasizes that neutronics and thermal hydraulics calculations are coupled only through temperature and power distributions. The whole-core power distributions define the heat source distributions for calculations with HeatWave. The resulting temperature distributions are used in calculations with SCALE.

The specific interfaces between the applied functional modules are also indicated in Fig. 1. The computational scheme combines the available capabilities of the SCALE Monte Carlo (whole-core analysis level) and deterministic functional modules (lattice level) with the HeatWave finite element solver.

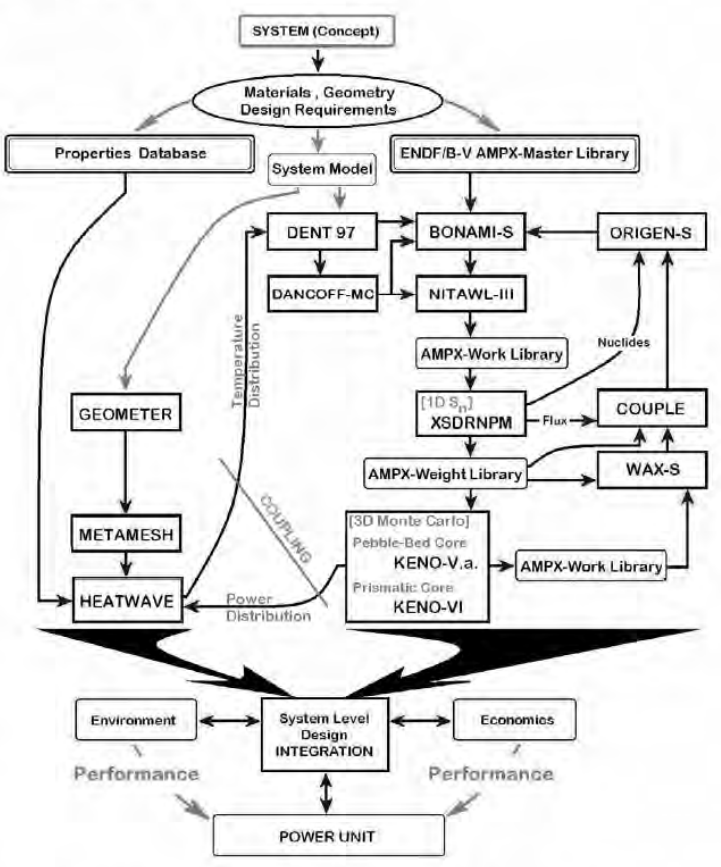

Fig.1. Computational modeling of the VHTR system.

As has already been noted, in the present analysis, at the lattice physics level, the resonance self-shielding calculations are performed accounting for double heterogeneity effects by so-called Dancoff correction factors. ${ }^{11}$ The Dancoff-based approach has successfully been implemented for and used in many parametric studies of VHTRs. ${ }^{4,11,13,14}$

The evaluated sensitivity of the VHTR configurations to Dancoff correction factors is shown in Fig. 2. The sensitivity is expressed in terms of the core reactivity levels as a function of Dancoff correction factor. To account for both VHTR core concepts, the analysis was performed using all LEU-HTR PROTEUS cores as well as the HTR-10 and the HTTR cores as the typical designs. ${ }^{6,7,15}$ This broad set of the considered existing core configurations allowed to determine the typical ranges of Dancoff correction factors for both core concepts and translate them to the core reactivity levels as illustrated in

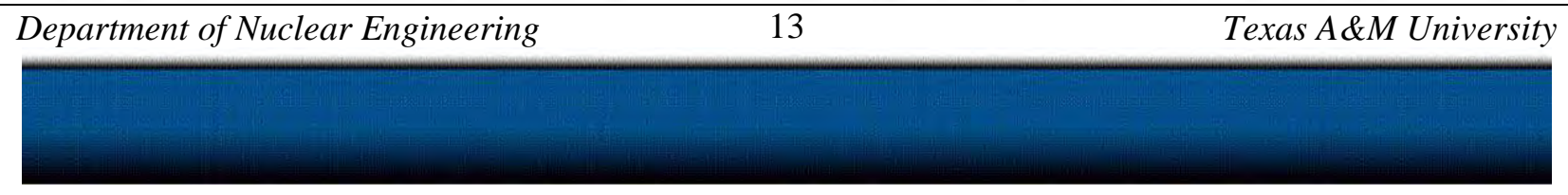




\section{Utilization of MAs as a Fuel Component for Ultra-Long Life} VHTR Configurations: Designs, Advantages and Limitations

\section{Project 05-094}

\section{Final Scientific/Technical Report}

Fig. 2 by the corresponding gray areas. Maximum and minimum sensitivity limits are found by varying Dancoff factors to their limits for each of the considered configurations. The existing isothermal benchmark cases allowed accounting for elevated temperatures.

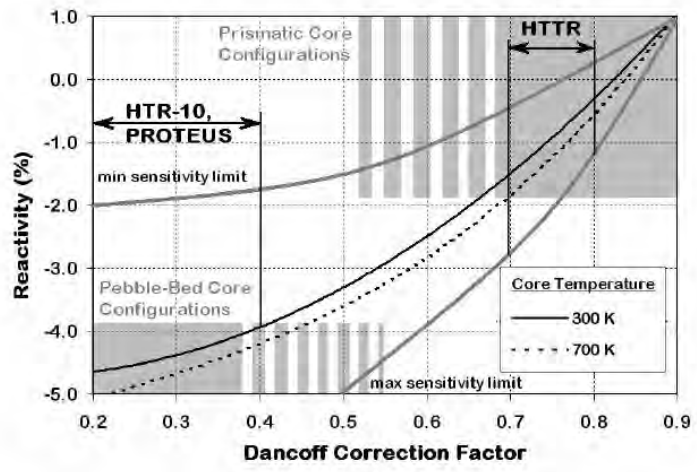

Fig. 2. VHTR reactivity as a function of Dancoff factor:

Figure 2 confirms that, although double heterogeneity effects are less pronounced in prismatic cores than in pebble-bed cores, their significance for both is clear. Taking into consideration both core design concepts, the overall accounted sensitivity span is $6 \%$ per factor of 3.5 in varying Dancoff correction factors.

Application of the coupled hybrid Monte Carlodeterministic methodology should facilitate and assure realistic performance evaluations. ${ }^{3,12}$ The key technical issues can be addressed and resolved by implementing computationally efficient automated modeling procedures and sequences, combining Monte Carlo and deterministic approaches, developing and applying realistic $3 \mathrm{D}$ coupled neutronics-thermal-hydraulics models with multiheterogeneity treatments, developing and performing experimental/computational benchmarks for model verification and validation, analyzing uncertainty effects and error propagation. A special effort should be made to verify that the computational modeling is consistent and realistic. Following the proposed computational modeling approach as shown in Fig. 1, the results describe performance of the entire VHTR power unit and allow conclusions regarding the configuration's feasibility, performance and possible directions for further analysis and development.

\section{VALIDATION AND VERIFICATION}

To assure comprehensive, realistic assessment of the VHTR designs and their performance characteristics targeting passive safety confirmation, the adequacy of the applied computational methods and models used to evaluate performance must be supported by comparisons with experimental data covering appropriate ranges of conditions. Validation data are available from power reactors as well as past critical experiments. The experimental data for validation of the high-fidelity coupled hybrid calculations are anticipated from the existing benchmark programs in the near future. ${ }^{15}$

As the basis for development of the benchmark test problems, the project uses the actual test results and developed benchmark problems of the LEU-HTR PROTEUS Program (pebble-bed criticality experiments), the HTTR Program (prismatic core design, reactor experiments), and the HTR-10 Program (pebble-bed core design, reactor experiments). ${ }^{6,7,15}$

Figure 3 illustrates the developed VHTR whole-core models describing the HTR-10 and HTTR cores. Besides modeling validation and verification studies, these models are used to represent small-scale VHTRs in studies confirming that the considered VHTR configurations exhibit realistic performance characteristics.

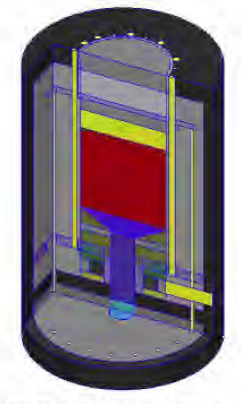

VHTR Pebble-Bed Model Benchmark Configuration (HTR-10)

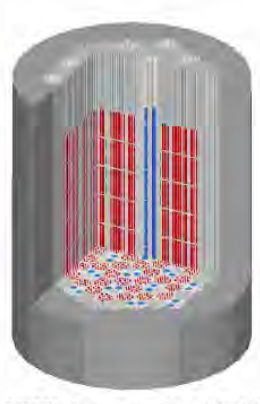

VHTR Prismatic Core Model Benchmark Configuration (HTTR)
Fig. 3. 3D whole-core models of HTR-10 and HTTR.

Table II summarizes the performed benchmark analysis. The provided data validate the developed $3 \mathrm{D}$ whole-core models and hybrid calculations using the SCALE code system. Up to date, there are no experimental data available to validate the coupled hybrid modeling approach involving the SCALE and HeatWave code systems simultaneously as shown in Fig. 1. It must be emphasized that the presented modeling results are obtained using general material properties and standard SCALE cross section libraries without any special tailoring to specific systems. The obtained calculated values are compared to the available experimental results from the corresponding International Benchmark Programs.

According to the program reports, the code-toexperiment discrepancy less than $10 \%$ is generally targeted as successful validation of a particular code applicability. ${ }^{15}$ Observed agreement with the available HTTR and HTR-10

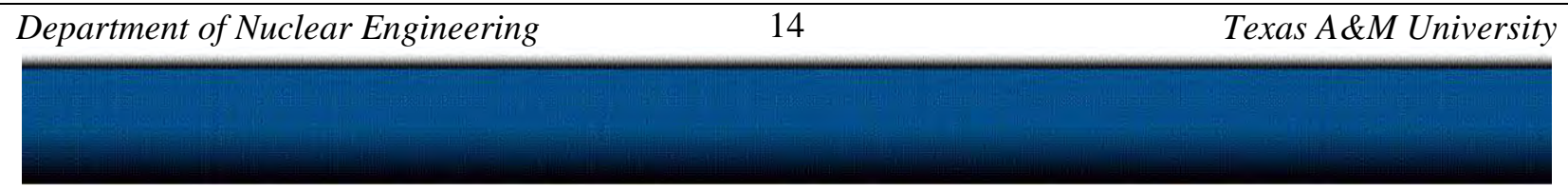




\section{Utilization of MAs as a Fuel Component for Ultra-Long Life} VHTR Configurations: Designs, Advantages and Limitations

\section{Project 05-094}

\section{Final Scientific/Technical Report}

data in the present analysis confirms adequate representation and applicability of the modeling approach. The larger deviation of computational results from experimental values for the HTTR cores with inserted control rods is due to limitations of the available information describing details of the specific control systems. The computed value of the isothermal temperature coefficient deviates by $13 \%$ from the corresponding experimental value. However, the experimental value is within the standard deviation limits of the computational result. Therefore, the discrepancy of $13 \%$ can be explained by insufficient accuracy of the performed modeling in this case. The larger discrepancies of the HTR-10 calculations are due to least amount of information available describing the system and its operation details.

TABLE II

VHTR Modeling Validation

(a) Prismatic Block Core Configuration

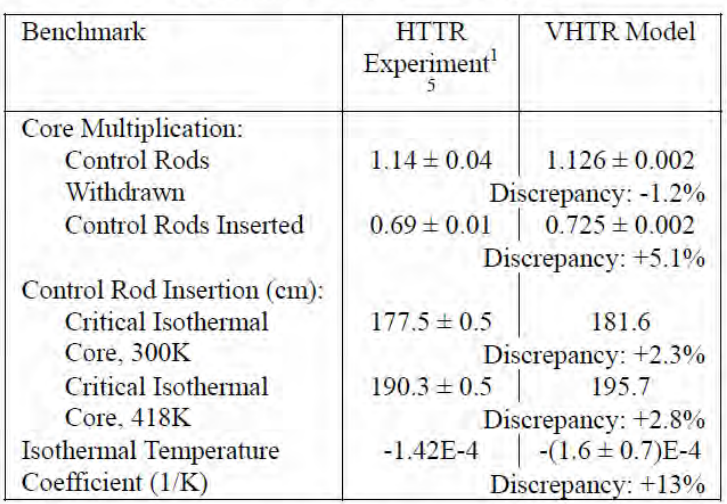

(b) Pebble Bed Core Configuration

\begin{tabular}{|l|c|c|}
\hline \multirow{2}{*}{$\begin{array}{l}\text { Isothermal Core } \\
\text { Temperature }\end{array}$} & \multicolumn{2}{|c|}{ Core Multiplication } \\
\cline { 2 - 3 } & $\begin{array}{c}\text { HTR-10 } \\
\text { China }^{15}\end{array}$ & VHTR Model \\
\hline $293 \mathrm{~K}$ & 1.1358 & $1.256 \pm 0.002$ \\
$393 \mathrm{~K}$ & \multicolumn{2}{|c|}{ Discrepancy: $+11 \%$} \\
$523 \mathrm{~K}$ & 1.1262 & $1.235 \pm 0.002$ \\
& \multicolumn{2}{|c|}{ Discrepancy: +9.7\% } \\
\hline
\end{tabular}

\section{ACTINIDE POTENTIAL IN THE VHTR}

Pebble-bed and prismatic core designs for VHTRs are characterized by significant flexibility in component configuration, fuel utilization and management. Consequently, it should be possible to enhance fissile properties of MA by neutron spectrum shifting through configuration adjustments.
Figure 4 compares properties of MAs and spectrum shifting capabilities of the VHTRs with pebble-bed cores. In this example, the spectrum shifting is achieved by varying moderator-to-fuel $(\mathrm{M} / \mathrm{F})$ pebble ratio in the reference core. Arrows show changes in dominant energies of neutrons inducing fission.

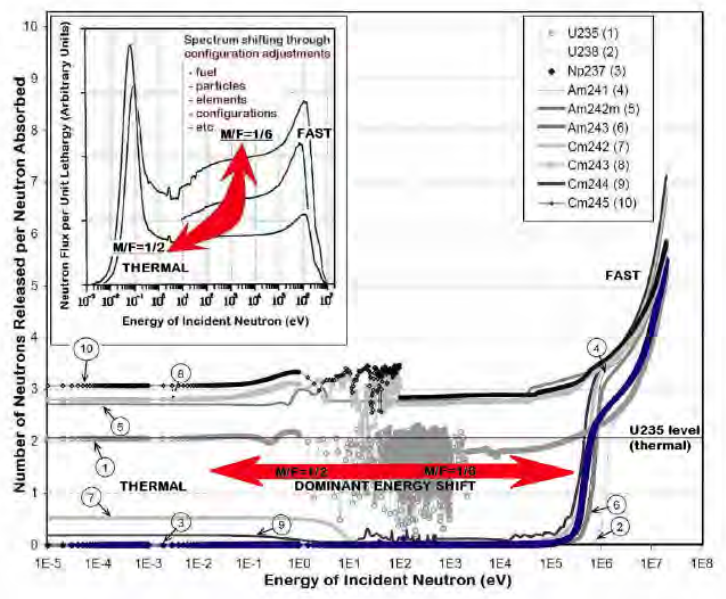

Fig. 4. Spectrum shifting in VHTRs and properties of MAs.

The principal mechanism being envisioned to achieve ultra-long life systems is an enhanced involvement of selfgenerated fissile compositions based on a spent LWR fuel to the energy generation process. As illustrated in Fig. 4, depending on neutron spectra in the considered core configurations (dominant neutron energies), neptunium, americium and curium may serve as burnable poisons or fuel materials contributing to small reactivity swings (selfstabilization) over prolonged irradiation periods.

\section{CONCLUSIONS}

The suggested coupled hybrid Monte Carlo deterministic approach offers consistent analysis of the advanced VHTR configurations taking into account their reactor physics features and performance characteristics. The developed whole-core/system 3D VHTR models passed the experimental benchmark testing for both core concepts at isothermal core temperatures. The presented results are obtained using general material properties and standard cross section libraries without any special tailoring to specific systems. Currently available experimental data do not allow validation of the complete coupled hybrid modeling approach.

Although to be further refined, studies of actinidefueled VHTR configurations indicate promising performance characteristics. They are being analyzed and compared taking into account the existing systems and the 


\section{Utilization of MAs as a Fuel Component for Ultra-Long Life} VHTR Configurations: Designs, Advantages and Limitations

\section{Project 05-094}

\section{Final Scientific/Technical Report}

Generation IV VHTR concepts with estimated characteristics.

The research effort enhances capabilities of the Generation IV VHTR and transforms it to a technology that can deliver electricity, hydrogen, and assist in spent fuel management while being inherently safe, environmentally friendly, and proliferation resistant. It is also going to be cost effective due to actinide recycle, high burn-up and reactor lifetime long autonomous operation expanding deployment to developing countries and remote areas.

\section{ACKNOWLEDGMENTS}

This paper is based upon work supported by the U.S Department of Energy under Award Number DE-FC0705ID14655 (05-094).

\section{DISCLAIMER}

This paper was prepared as an account of work sponsored by an agency of the United States Government. Neither the United States Government nor any agency thereof, nor any of their employees, makes any warranty, express or implied, or assumes any legal liability or responsibility for the accuracy, completeness, or usefulness of any information, apparatus, product, or process disclosed, or represents that its use would not infringe privately owned rights. Reference herein to any specific commercial product, process, or service by trade name, trademark, manufacturer, or otherwise does not necessarily constitute or imply its endorsement, recommendation, or favoring by the United States Government or any agency thereof. The views and opinions of authors expressed herein do not necessarily state or reflect those of the United States Government or any agency thereof.

\section{NOMENCLATURE}

$\begin{array}{ll}\text { AFCI } & \text { - Advanced Fuel Cycle Initiative } \\ \text { HLW } & \text { - High Level Wastes } \\ \text { MA } & \text { - Minor Actinides } \\ \text { NERI } & \text { - Nuclear Energy Research Initiative } \\ \text { P\&T } & \text { - Partitioning and Transmutation } \\ \text { VHTR } & \text { - Very High Temperature Reactor }\end{array}$

\section{REFERENCES}

1. "Report to Congress on Advanced Fuel Cycle Initiative: The Future Path for Advanced Spent Fuel Treatment and Transmutation Research", 03GA50439-06, January, 2003, Office of Nuclear Energy, Science, and Technology, U.S. DOE (2003).
2. "Generation IV Nuclear Energy Systems: Ten Year Program Plan", March 2005, Office of Advanced Nuclear Research, DOE Office of Nuclear Energy, Science, and Technology, U.S. DOE (2005).

3. P. V. TSVETKOV, R. R. HART, "Hybrid Monte Carlo-Deterministic Analysis of Fission Fragment Magnetic Collimator Reactors", Proc. Intern. Conf. on Monte Carlo Methods, Chattanooga, Tennessee, April 17-21, 2005, Amer. Nucl. Soc., Lagrange Park, IL. (2005).

4. P. V. TSVETKOV, "Basic Reactor Physics Parameter Study of the Pebble-Bed Nuclear Reactor System", IRI-131-97-002, Jan., 1997, TUDelft, the Netherlands (1997)

5. R. PLUKIENE, D. RIDIKAS, "Modeling of HTRs with Monte Carlo: from a Homogeneous to an Exact Heterogeneous Core with Microparticles", An. Nucl. En., 30, 1573, (2003).

6. R. CHAWLA, D. MATHEWS, "LEU-HTR PROTEUS Calculational Benchmark Specifications", TM-41-90-32. PSI, Villigen, Switzerland (1990).

7. "Critical Experiments and Reactor Physics Calculations for Low-Enriched HTGRs", IAEATECDOC-1249, International Atomic Energy Agency, Vienna, Austria (2001)

8. J. F. BRIESMEISTER, ed., "MCNP, A General Monte Carlo N-Particle Transport Code, Version 5, User's Manual", LA-UR-03-1987, Los Alamos National Laboratory (2003).

9. "SCALE: A Modular Code System for Performing Standardized Computer Analyses for Licensing Evaluation", ORNL/TM-2005/39, Ver. 5, April 2005, Oak Ridge National Laboratory (2005).

10. S. HUMPHRIES, Jr., Field Solutions on Computers, CRC Press, Boca Raton, Florida (1997).

11. J. VALKO, P. V. TSVETKOV, J. E. HOOGENBOOM, "Calculation of the Dancoff Factor for Pebble-Bed Reactors", Nucl. Sci. Eng., 135, 304 (2000).

12. P. V. TSVETKOV, A. N. CHMELEV, N. I. GERASKIN, "Complex Multi-Task Analysis of the Nuclear Reactor Systems with the DENT-97 Computer Code System", Proc. Intern. Conf. on the Phys. of Nucl. Sci. and Tech., Long Island, New York, October 5 - 8, 1998, 2, 851. Amer. Nucl. Soc. Lagrange Park, IL. (1998).

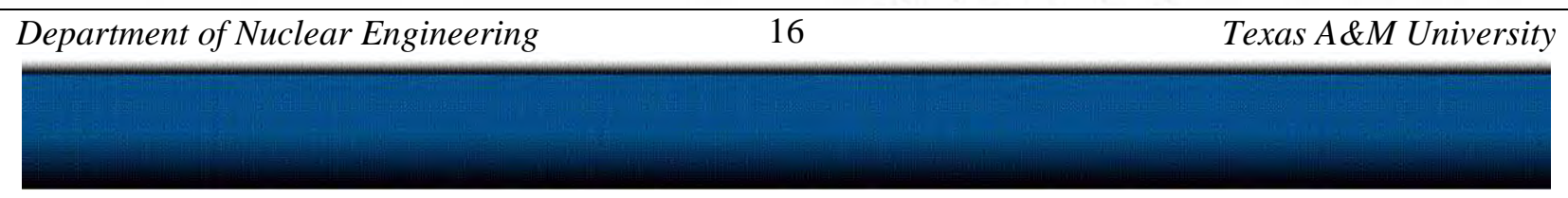




\section{Utilization of MAs as a Fuel Component for Ultra-Long Life} VHTR Configurations: Designs, Advantages and Limitations

Project 05-094

Final Scientific/Technical Report

13. P. V. TSVETKOV, J. E. HOOGENBOOM, "Reactivity Effects in a HTGR due to Variations of Basic PebbleBed Characteristics", Trans. Amer: Nucl. Soc. 91, 559 (2004).

14. A. M. OUGOUAG, H. D. GOUGAR. W. K. TERRY. R. MPHAHLELE, K. N. IVANOV, "Optimal Moderation in the Pebble-Bed Reactor for Enhanced Passive Safety and Improved Fuel Utilization", Proc. PHYSOR 2004, Chicago, Illinois, April 25-29, 2004, Amer. Nucl. Soc., Lagrange Park, IL. (2004),

15. "Evaluation of High Temperature Gas-cooled Reactor Performance: Benchmark Analysis Related to Initial Testing of the HTTR and HTR-10", IAEA-TECDOC1382, International Atomic Energy Agency. Vienna, Austria (2003). 


\section{BENCHMARK EFFORTS TO SUPPORT STUDIES OF ADVANCED VHTRs}

The Very High Temperature Reactor (VHTR) is the leading candidate for the reactor component of the Next Generation Nuclear Plant (NGNP). This is because the VHTR demonstrates great potential in improving safety characteristics, being economically competitive, providing a high degree of proliferation resistance, and producing high outlet temperatures for efficient electricity generation and/or other high temperature applications, most notably hydrogen production. In addition, different fuel types can be utilized by VHTRs, depending on operational goals. In this case, the recovery and utilization of the valuable energy left in LWR fuel in order to create ultra long life single batch cores by taking advantage of the properties of TRU fuels.

This paper documents the initial process in the study of TRU fueled VHTRs, which concentrates on the verification and validation of the developed whole-core 3D VHTR models. Many of the codes used for VHTR analysis were developed without a full appreciation of the importance of randomness in particle distribution.

With this in mind, the SCALE code system was chosen as the computational tool for the study. It provides the opportunity of utilizing SCALE versions 5.0 and 5.1, making it possible to compare and analyze different techniques accounting for the double heterogeneity effects associated with VHTRs.

Startup physics results for Japan's High Temperature Test Reactor (HTTR) were used for experiment-to-code benchmarking. MCNP calculations were employed for code-to-code benchmarking. Results and analysis are included in this paper. 


\title{
BENCHMARK EFFORTS TO SUPPORT STUDIES OF ADVANCED VHTRS
}

\author{
David E. Ames II, Pavel V. Tsvetkov \\ Department of Nuclear Engineering \\ Texas A\&M University \\ 129 Zachry Engineering Center, MS 3133 TAMU, \\ College Station, Texas 77843-3133, USA \\ Phone: $979 / 845-7078$ \\ Fax: 979/845-6443 \\ E-mail: Tsvetkov@tamu.edu
}

\begin{abstract}
The Very High Temperature Reactor (VHTR) is the leading candidate for the reactor component of the Next Generation Nuclear Plant (NGNP), This is because the VHTR demonstrates great potential in improving safety characteristics. being economically competitive, providing a high degree of proliferation resistance, and producing high outlet temperatures for efficient electricity generation and/or other high temperature applications, most notably hydrogen production. In addition, different fuel types can be utilized by VHTRs. depending on operational goals. In this case, the recovery and utilization of the valuable energy left in LWR fuel in order to create ultra long life single batch cores by taking advantage of the properties of TRU fuels.

This paper documents the initial process in the study of TRU fueled VHTRs, which concentrates on the verification and validation of the developed whole-core 3D VHTR models Many of the codes used for VHTR analysis were developed without a full appreciation of the importance of randomness in particle distribution.

With this in mind, the SCALE code system was chosen as the computational tool for the study. It provides the opportunity of utilizing SCALE versions 5.0 and 5.1, making it possible to compare and analyze different techniques accounting for the double heterogeneity effects associated with VHTRs.

Startup physics results for Japan's High Temperature Test Reactor (HTTR) were used for experiment-to-code benchmarking. MCNP calculations were employed for code-tocode benchmarking. Results and analysis are included in this paper.
\end{abstract}

NOMENCLATURE

HTGR - High Temperature Gas-cooled Reactor

HTIR - High Temperature Test Reactor

JAERI - Japan Atomic Energy Research Institute

LWR - Light Water Reactor

NERI - Nuclear Energy Research Initiative

NGNP - Next Generation Nuclear Plant

TRU - Transuranium Nuclides

VHTR - Very High Temperature Reactor

\section{INTRODUCTION}

The HTTR of the Japan Atomic Energy Research Institute (JAERI) is a graphite-moderated and helium gas-cooled reactor with $30 \mathrm{MW}$ in thermal output and outlet coolant temperature of $850 \mathrm{C}$ for rated operation and $950 \mathrm{C}$ for high temperature test operation [1].

The HTTR was selected for the benchmark study because of the reference material available. The reference material provided: general design features, reactor layout, core configuration, material properties, and other relevant information to develop the computational model. Also supplied were actual experimental test results to be utilized for experiment-to-code benchmarks and several analytical code results for code-to-code benchmark tests [1]. Taking advantage of the performed benchmark studies, the HTIR configurations are also used as initial prototype designs representing smallscale VHTR concepts.

\section{VHTR PRISMATIC CORE MODEL}

The VHTR model is a nearly explicit representation of the existing HTTR core configuration. The model was created in the SCALE [2] code system ntilizing the CSAS6/KENO-VI module, which allows flexible geometry representations. The 


\section{Utilization of MAs as a Fuel Component for Ultra-Long Life VHTR Configurations: Designs, Advantages and Limitations}

\section{Project 05-094}

Final Scientific/Technical Report

geometry package in KENO-VI is capable of modeling any volume that can be constructed by quadratic equations (second order surfaces).

In addition to the model description provided here, complete details of material compositions and geometry specifications can be found by Ames [3] and Iyoku [4].

\subsection{Fuel Assembly Block}

The fuel assembly block consists of 4 main components: hexagonal graphite block, fuel rods, burnable poison $\left(\mathrm{C}, \mathrm{B}_{4} \mathrm{C}\right)$ rods, and helium coolant channels. The core contains 150 fuel assembly blocks, which construct 30 columns ( 5 fuel blocks stacked vertically) within the core. Although these fuel blocks share the same general characteristics, there are subtle differences between them. For instance, individual fuel blocks can have different fuel enrichments (12 enrichments: $3.3 \%-$ $9.8 \%$ ), one of two types of burnable poison rods (H-I or H-II), and either 31 or 33 annular fuel rods. This translates to 48 different types of fuel assembly blocks in the core, which can be identified by fuel enrichment, number of fuel compacts, and type of burnable poison $\left(\mathrm{C}, \mathrm{B}_{4} \mathrm{C}\right)$ rod.

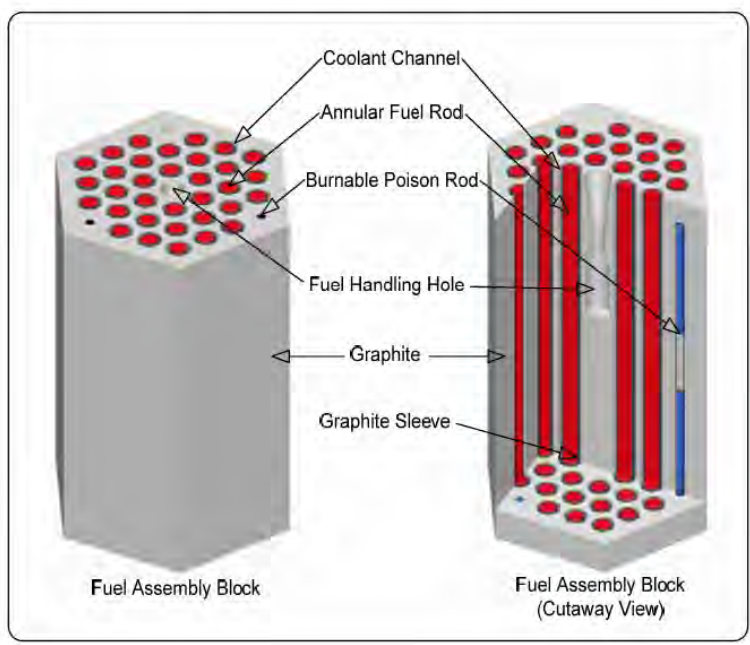

Figure 1. Fuel assembly block (KENO 3D).

The general parameters shared by the fuel blocks are fuel rods, coolant channels, and two burnable poison $\left(\mathrm{C}, \mathrm{B}_{4} \mathrm{C}\right)$ rods, which are assembled in a hexagonal graphite block to create a pin-in-block type assembly. The fuel graphite block is $36 \mathrm{~cm}$ in width across the flats and $58 \mathrm{~cm}$ in height. The block has either 31 or 33 vertical borings (depending on number of fuel rods) with a diameter of $4.1 \mathrm{~cm}$ for placement of the annular fuel rods. In addition, each fuel graphite block has three burnable poison $\left(\mathrm{C}, \mathrm{B}_{4} \mathrm{C}\right)$ insertion holes measuring $50 \mathrm{~cm}$ in height and $1.5 \mathrm{~cm}$ in diameter. Two are loaded with burnable poison $\left(\mathrm{C}, \mathrm{B}_{4} \mathrm{C}\right)$ rods, while the third is left empty. In the center of each block is a fuel handling hole.
The annular fuel rod consists of a fuel compact with an outer graphite sleeve. The graphite sleeve has an inner diameter of $2.6 \mathrm{~cm}$, outer diameter of $3.4 \mathrm{~cm}$, and height of 58 $\mathrm{cm}$. The compact has an inner diameter of $1.0 \mathrm{~cm}$, outer diameter of $2.6 \mathrm{~cm}$, height of $54.6 \mathrm{~cm}$, and is composed of fuel particles imbedded in a graphite matrix. The rods are inserted into the vertical holes of the graphite fuel block. Helium gas coolant flows through the gaps that are created between the holes and the rods.

The burnable poison rod is $1.4 \mathrm{~cm}$ in diameter and $50 \mathrm{~cm}$ in height. It is made up of two neutron absorber sections (20 $\mathrm{cm}$ in height) separated by a graphite section (10 cm in height). Fig. 1 illustrates an example of the fuel assembly block that was used in the computational model.

\subsection{Replaceable Reflector Blocks}

The replaceable reflector block has the same external form as the fuel assembly block, $36 \mathrm{~cm}$ in width across the flats and $58 \mathrm{~cm}$ in height with a handling hole in the center of the block. There are two types of reflector blocks; one being a solid graphite block, and the other having helium coolant channels.

The reflector blocks with the coolant channels are stacked directly above and below the fuel assembly blocks. This creates a fuel column, being composed of 2 replaceable reflector blocks on top, 5 fuel assembly blocks in the middle, and 2 replaceable reflector blocks on the bottom (total of 9 blocks). The blocks have the same dimensions as the fue] graphite block within the same column, with the exception of not having the three burnable poison insertion holes. This allows the helium gas to flow into the core, through the fuel assemble blocks, and then exit the core. Examples of the replaceable reflector blocks are provided in Fig. 2.

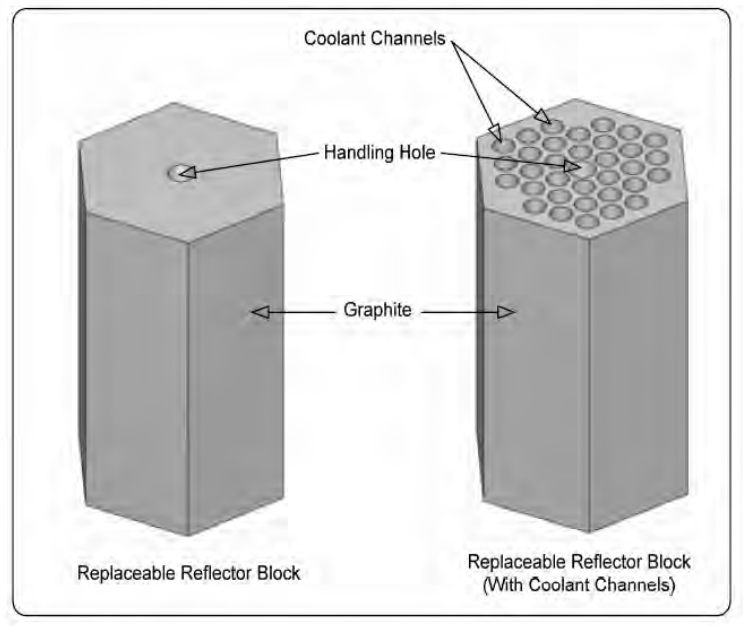

Figure 2. Replaceable reflector blocks (KENO 3D).

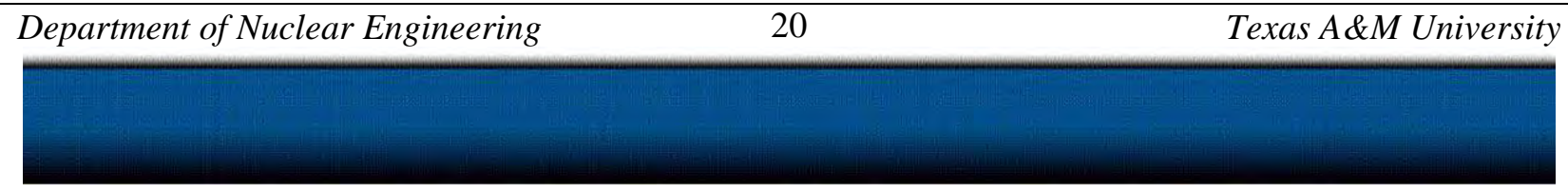




\section{Utilization of MAs as a Fuel Component for Ultra-Long Life VHTR Configurations: Designs, Advantages and Limitations}

\section{Project 05-094}

Final Scientific/Technical Report

\subsection{Control Rod Guide Block}

There are 144 control rod guide blocks in the core. They form 16 control rod guide columns in the core $(9$ blocks stacked vertically). The block consists of a hexagonal graphite block with three large vertical borings. Just as the fuel block, it is $58 \mathrm{~cm}$ in height and $36 \mathrm{~cm}$ in width across the flats

The holes created by the borings have a $12.3 \mathrm{~cm}$ diameter and extend through the entire length of the block. Two of the holes are used for the control rods to pass through, while the third is left empty to serve as the reserve shutdown system. There is a fuel handling bole in the center of each block.

There are 16 pairs of control rods, 7 in the active core and 9 in the replaceable reflector region. A pair of control tods is inserted into the holes of a control rod guide column. Each control rod consists of 10 annular neutron absorber section $(\mathrm{B} 4 \mathrm{C} / \mathrm{C}$ ) having an inner diameter of $6.5 \mathrm{~cm}$, outer diameter of $10.5 \mathrm{~cm}$, and height of $29 \mathrm{~cm}$. Each section is separated by 2.2 $\mathrm{cm}$, which translates to an overall rod height of $310 \mathrm{~cm}$.

The annular absorber sections are incased by a sleeve constructed of Alloy $800 \mathrm{H}$ with a thickness of $0.35 \mathrm{~cm}$, Fig. 3 illustrates an example of the control rod guide block with control rods inserted. The figures come directly from the computational model.

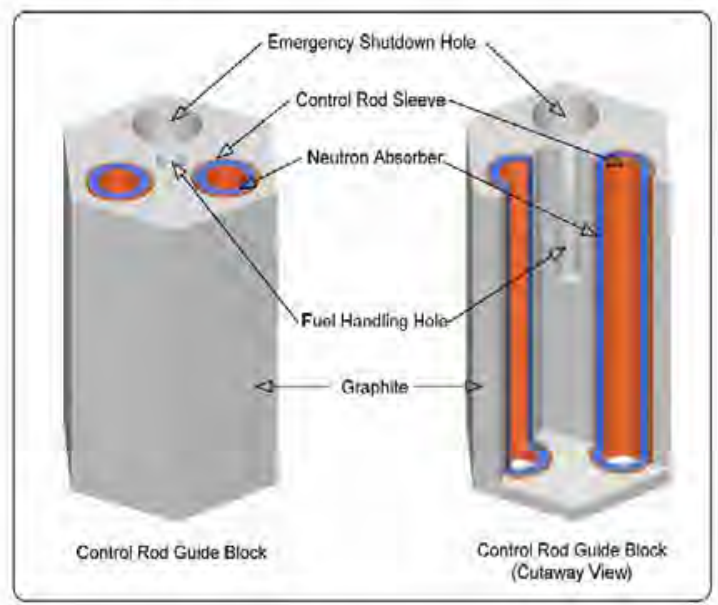

Figure 3. Control rod guide block (KENO 3D).

\subsection{Irradiation Block}

The irradiation block is identical to the control rod guide block, but the holes in the irradiation block are only used for nuclear instrumentation. There are 27 irradiation blocks that makeup three irradiation columns ( 9 blocks vertically stacked per column). In the case of the model the holes in the blocks were left empty.

\subsection{Building the Model}

The four types of prismatic hexagonal blocks just described are stacked vertically to make a column. The columns are classified by the type of block, making four types of columus:

1) firel assembly block column,

2) control rod block column,

3) replaceable reflector block column, and

4) irradiation block columm.

Each column consists of nine blocks, resulting in a height of $522 \mathrm{~cm}$. The columns are then arranged side-to-side in a hexagonal anray to construct the model. A horizontal crosssection of the model showing the arrangement of the columns is provided in Fig 4 .

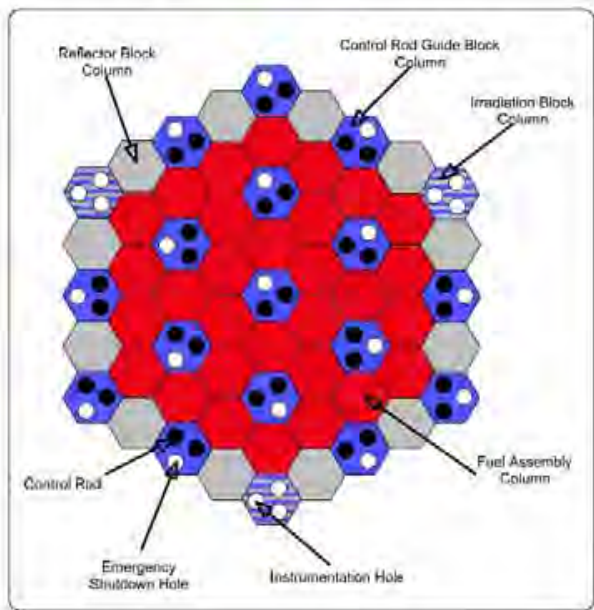

Figure 4. Hexagoual anray of prismatic coltumns.

The core components (array of prismatic hexagonal columns) are horizontally surrounded by a permanent reflector having a diameter of $430 \mathrm{~cm}$. The structures outside of the permanent reflector region were neglected in the calculation models. A cross-sectional view of the model is shown in Fig. 5.

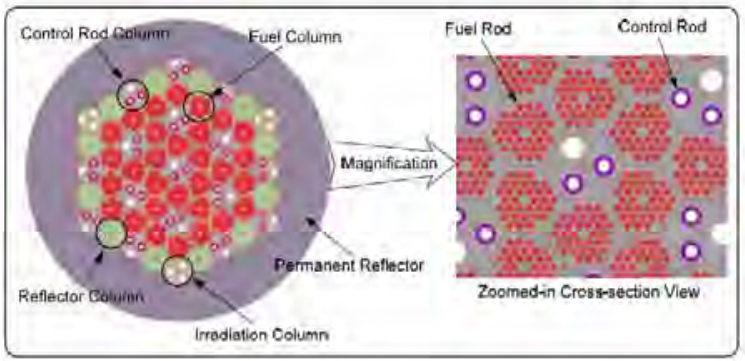

Figure 5. Horizontal cross section of the VHTR (KENO 3D).

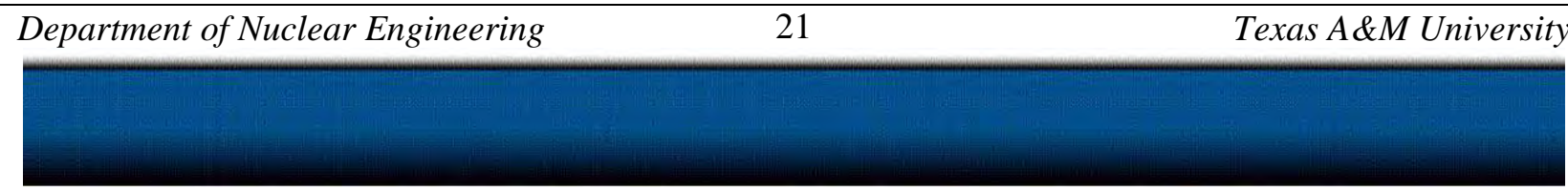




\section{Utilization of MAs as a Fuel Component for Ultra-Long Life VHTR Configurations: Designs, Advantages and Limitations}

The complete VHTR model has an overall height of 522 $\mathrm{cm}$ and a diameter of $430 \mathrm{~cm}$. The fueled region or active corc is $290 \mathrm{~cm}$ in height and $230 \mathrm{~cm}$ in elfective diameter. Fig. 6 is a $3 D$ cutaway view of the VHTR model. which shows the active core. In this particular figure the control rods are fully inserted in the core

\subsection{Homogenized Fuel Region}

The computational model is a very detailed model of the HTTR in which practically all components are modeled explicitly. with the exception of the fuel compacts. The fuel compact consists of TRISO coated fuel particles imbedded within a graphite matrix in the form of an annular rod. Each compact contains about 182,000 coated fiel particles, which consist of a spherical fuel kernel of low enriched UO2 with TRISO coating. The coating is made up of a low-density. porous pyrolytic cabon ( $\mathrm{PyC}$ ) buffer layer adjacent to the fuel kemel, followed by lngh density isotropic PyC layer. a silicon carbide banrier layer and a limal outer PyC layer. Table 1 lists the properties of the TRISO fuel particles.

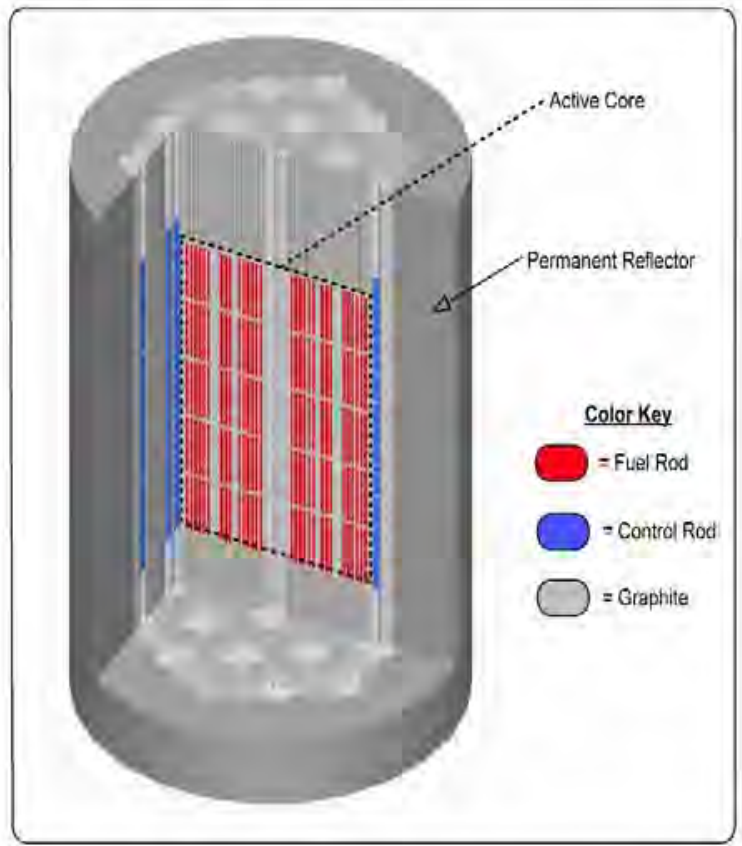

Figure 6. Three dimensional cutaway view (KENO 3D)

In the computational model the TRISO fuel particles are homogenized with the graphite matrix of the fuel compacts. An illustration showing an example of the TRISO fuel particle, the fiel compact (with section removed to show imbedded fiel particles), and the homogenized fuel compact is provided in Fig. 7.

Table 1 TRISO Fuel Particles
\begin{tabular}{|l|c|c|c|}
\hline TRISO & Material & Density (g/cc) & Radlus $(\mathbf{c m})$ \\
\hline Fuel kernel & UO2 & 10.41 & 0.0300 \\
\hline 1st coating & PyC & 1.14 & 0.0359 \\
\hline 2nd coating & PyC & 1.89 & 0.0390 \\
\hline 3rd coating & SiC & 3.20 & 0.0419 \\
\hline 4th coating & PyC & 1.87 & 0.0465 \\
\hline
\end{tabular}

The VHTR model must account for the double heterogeneity affects that accompany the system. The annular fuel compacts, which are composed of helerogeneous fuel particles randomly dispersed in a moderator matrix, make up the first level of heterogeneity. The fuel compacts themselves are also heterogeneous with fuel and moderator or reflector regions, forming the second level of heterogeneity.

Past research [5] has shown that effectively accounting for heterogeneity effects in VHTR systems is very important and must be accounted for accordingly. Newly available in SCALE version 5.1 is the capability to account for this phenomenon using the DOUBLEHEI unt cell treatment. Alternatively, the DOUBLEHET leature can be bypassed and instead a Dancoff correction factor can be manually entered, providing an alternative option to account for the double heterogeneity effects, This procedure was performed to provide a comparison to the DOUBLEHET feature of SCALE 5.1. To accomplish this, the Dancoff factor was independently detenmined by using the code system DANCOFF-MC [6]

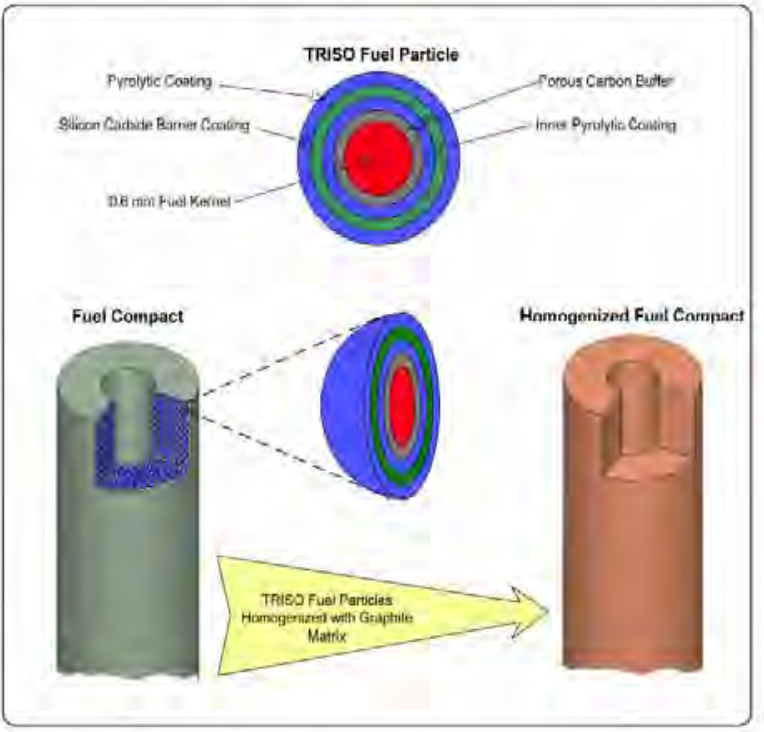

Figure 7. TRISO fuel particle and fuel compact.

Table 2 provides a comparison of the results for the two different treatments accounting for the heterogeneity effects 


\section{Utilization of MAs as a Fuel Component for Ultra-Long Life} VHTR Configurations: Designs, Advantages and Limitations

As seen, a higher degree of accuracy was accomplished with the DOUBLEHET model, therefore it was chosen as the VHTR model.

Table 2 HTTR Criticality Calculations Using Externally Determined Dancoff Factor (Dancoff-MC) and SCALE 5.1 DOUBLEHET Treatment

\begin{tabular}{|l|l|l|}
\hline VHTR Model & k-eff & Error (\%) \\
\hline Experimental (HTTR) & 1.1363 & - \\
\hline SCALE 5.0 (with DANCOFF-MC) & 1.1122 & $2.12 \%$ \\
\hline SCALE 5.1 (with DOUBLEHET) & 1.1368 & $0.04 \%$ \\
\hline
\end{tabular}

\subsection{Map of the VHTR Core}

Being that the fuel assembly blocks can have a number of different arrangements: 31 or 33 fuel rods, 12 different uranium fuel enrichments, and two types of burnable poison rods (H-I and $\mathrm{H}-\mathrm{II}$ ); a system to identify their position in the core was developed. It separates the fuel columns into four fuel zones and assigns a number to the rows that construct the columns.

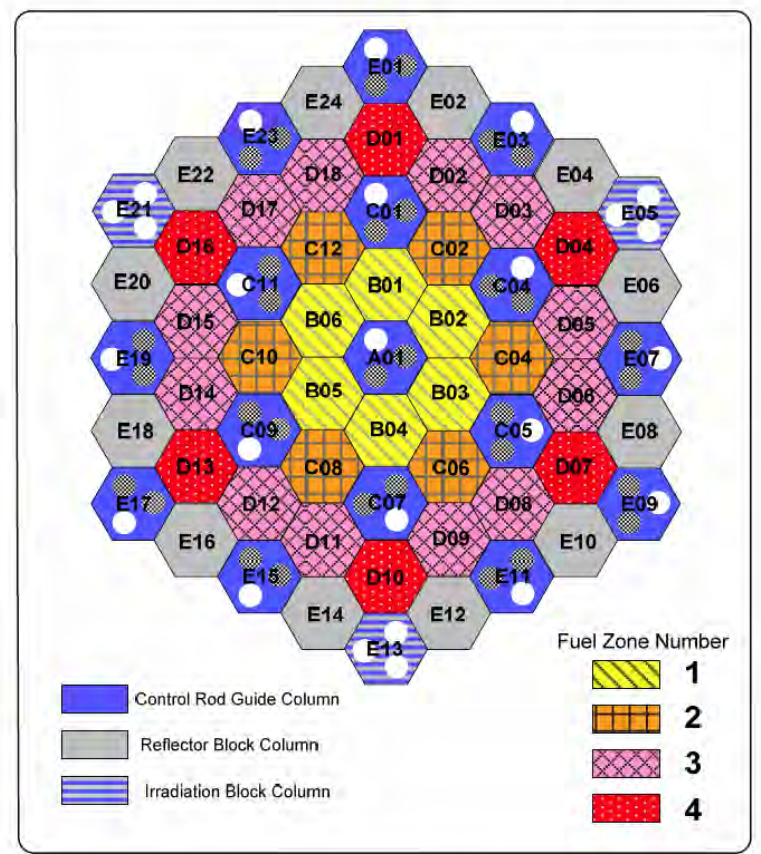

Figure 8. Map of the HTTR core.

There are five rows (representing the five fuel assembly blocks in the fuel column), the first being the top row and the fifth being the bottom row. In addition, it is important to know the radial positions in the core. This is accomplished by naming each hexagonal block. A map of the core is provided in
Fig. 8. Table 3 identifies were the fuel assembly blocks are placed.

\section{EXPERIMENT-TO-CODE BENCHMARK}

The benchmark problems are related to start-up core physics tests and include the analysis of the effective multiplication factor of fully loaded core with control rods fully withdrawn and fully inserted, control rod position at criticality, and isothermal temperature coefficient of reactivity. All calculations were performed for a core temperature of $300 \mathrm{~K}$ unless otherwise specified

The effective multiplication factor was evaluated with the control rods fully withdrawn and inserted. When the control rods are fully withdrawn, their upper limit is the upper face of the top reflector block of the core $(116 \mathrm{~cm}$ above the active core) with the exception of the six pair of control rods located on the perimeter of the core. The control rods of these six columns have an upper limit of $72.5 \mathrm{~cm}$, which translates to $43.5 \mathrm{~cm}$ above the active core. When the control rods are fully inserted, the lower ends of all the control rods are on the same plane with the bottom face of the active core.

Table 3 Core Arrangement

\begin{tabular}{|c|c|c|c|c|c|}
\hline \multirow{2}{*}{$\begin{array}{c}\text { Layer from } \\
\text { top fuel } \\
\text { block }\end{array}$} & \multirow{2}{*}{ Items } & \multicolumn{4}{|c|}{ Fuel zone number } \\
\hline & & 1 & 2 & 3 & 4 \\
\hline \multirow{3}{*}{1} & Uranium enrichment (wht. \%) & 6.681 & 7.82 & 9.358 & 9.81 \\
\hline & Number of fuel rods in graphite block & 33 & 33 & 31 & 31 \\
\hline & Type of burnable poisons & $\mathrm{H}-\mathrm{I}$ & $\mathrm{H}-\mathrm{I}$ & $\mathrm{H}-\mathrm{I}$ & $\mathrm{H}-\mathrm{I}$ \\
\hline \multirow{3}{*}{2} & Uranium enrichment (wht. \%) & 5.162 & 6.254 & 7.189 & 7.82 \\
\hline & Number of fuel rods in graphite block & 33 & 33 & 31 & 31 \\
\hline & Type of burnable poisons & $\mathrm{H}-\|$ & $\mathrm{H}-\|$ & $\mathrm{H}-\|$ & $\mathrm{H}-\|$ \\
\hline \multirow{3}{*}{3} & Uranium enrichment (wt. \%) & 4.29 & 5.162 & 5.914 & 6.254 \\
\hline & Number of fuel rods in graphite block & 33 & 33 & 31 & 31 \\
\hline & Type of burnable poisons & $\mathrm{H}-\| \mathrm{I}$ & $\mathrm{H}-\|$ & $\mathrm{H}-\|$ & $\mathrm{H}-\|$ \\
\hline \multirow{3}{*}{4} & Uranium enrichment (wot. \%) & 3.301 & 3.864 & 4.29 & 4.794 \\
\hline & Number of fuel rods in graphite block & 33 & 33 & 31 & 31 \\
\hline & Type of burnable poisons & $\mathrm{H}-\mathrm{I}$ & $\mathrm{H}-\mathrm{I}$ & $\mathrm{H}-\mathrm{I}$ & $\mathrm{H}-\mathrm{I}$ \\
\hline \multirow{3}{*}{5} & Uranium enrichment (wt. \%) & 3.301 & 3.864 & 4.29 & 4.794 \\
\hline & Number of fuel rods in graphite block & 33 & 33 & 31 & 31 \\
\hline & Type of burnable poisons & $\mathrm{H}-\mathrm{I}$ & $\mathrm{H}-\mathrm{I}$ & $\mathrm{H}-\mathrm{I}$ & $\mathrm{H}-\mathrm{I}$ \\
\hline
\end{tabular}

The control rod insertion depths were evaluated at the critical condition for the fully loaded core. All control rod insertion levels are adjusted on the same level except three pairs of control rods that are located at columns E03, E09, E17 of the most outer region in the side reflectors, as shown in Fig. 8. These three pairs of control rods are fully withdrawn for the calculation. When fully withdrawn, the upper limit is the upper face of the first replaceable reflector block $(116 \mathrm{~cm}$ above the active core). The control rod critical positions are measured from the bottom of the active core. Control rod insertion depths were calculated for core temperatures of $300 \mathrm{~K}, 418 \mathrm{~K}$ and $480 \mathrm{~K}$.

Isothermal temperature coefficients for the fully-loaded core were evaluated using the effective multiplication factors according to the following relationship: 


$$
\rho_{n}=\frac{k_{n+1}-k_{n}}{k_{n+1} \cdot k_{n}} \cdot \frac{1}{T_{n+1}-T_{n}},
$$

where:

$\rho_{n}$ : Temperature coefficient between $T_{n}$ and $T_{n+1}(\Delta k / k / K)$

$T_{n}$ : Core temperature at $n^{\text {th }}$ measurement $(K)$

$T_{n+1}$ : Core temperature at $n+1^{\text {th }}$ measurement $(K)$

$k_{n}$ : Effective multiplication factor at $T_{n}$

$k_{n+1}$ : Effective multiplication factor at $T_{n+1}$

The critical control rod positions are changed with temperature elevation in the real reactor operation. However, the control rod position is not changed in the calculation to obtain the reactivity difference.

\subsection{Results}

Following the established international benchmark program practices, in the present analysis $10 \%$ discrepancy between computed values and the available experimental values was considered as the model acceptability threshold. The results of the analysis showing comparison to experimental data are summarized in Table 4. Experimental data for prismatic core configurations are available from the HTTR program [1].

Table 4 Experiment-to-Code Benchmark Results

\begin{tabular}{|l|c|c|c|c|}
\hline \multicolumn{2}{|c|}{ Benchumark } & $\begin{array}{c}\text { VHTR model } \\
\text { (calculated) }\end{array}$ & $\begin{array}{c}\text { HTTR } \\
\text { (experimental) }\end{array}$ & $\begin{array}{c}\text { Error } \\
(\%)\end{array}$ \\
\hline Control Rods Fully Withdrawn & $\mathrm{k}$-eff & $1.1368 \pm 0.0023$ & $1.1363 \pm 0.041$ & 0.044 \\
\hline Control Rods Fully Inserted & $\mathrm{k}$-eff & $0.6858 \pm 0.0019$ & $0.685 \pm 0.010$ & 0.117 \\
\hline $\begin{array}{l}\text { Critical Insertion Depth } \\
\text { (core temperatuse } 300 \mathrm{~K})\end{array}$ & $\mathrm{cm}$ & 177.1 & $177.5 \pm 0.5$ & 0.225 \\
\hline $\begin{array}{l}\text { Critical Insertion Depth } \\
\text { (core temperatuse } 418 \mathrm{~K} \text { ) }\end{array}$ & $\mathrm{cm}$ & 189.9 & $190.3 \pm 0.5$ & 0.210 \\
\hline Temperature Coefficient & $\mathrm{dkk} / \mathrm{k} / \mathrm{K} /$ & $-1.45 \mathrm{E}-04$ & $-1.42 \mathrm{E}-04$ & 2.113 \\
\hline
\end{tabular}

Aside from the temperature coefficient, each of the benchmark cases is within $0.25 \%$ of the experimental values and fall within experimental error. The computed value of the isothermal temperature coefficient deviates by about $2 \%$ from the corresponding experimental value. However, the experimental value is within the standard deviation limits of the computational result. It is expected that increasing the sample size of the model would result in reducing the discrepancy to within the range of the other benchmark tests. However, for the benchmark calculations a maximum computational run time was set and higher accuracy results were not obtained in the present analysis.

The HTTR configuration with fully withdrawn control rods was chosen as a prototype VHTR configuration. The best agreement with experimental data was observed for that case. Table 5 summarizes basic reactor physics characteristics obtained for the prototype VHTR configuration
Table 5 Basic Reactor Physics (Withdrawn Control Rods)

\begin{tabular}{|c|c|c|c|}
\hline k-eff & $\begin{array}{c}\text { Fission-Inducing } \\
\text { Energy (eV) }\end{array}$ & $\begin{array}{c}\text { System Mean Free } \\
\text { Path (cm) }\end{array}$ & $\begin{array}{c}\text { Fission Neutron } \\
\text { Yield }\end{array}$ \\
\hline $1.1368 \pm 0.0023$ & $0.0814041 \pm 0.0002014$ & $2.94445 \pm 0.00121$ & $2.43872 \pm 0.00001$ \\
\hline
\end{tabular}

It is proposed that through configuration adjustments, spectrum shifting can be achieved, with the end result of improving fissile properties of TRU fuels. With that concept in mind, a preliminary analysis of the energy-dependent neutron flux in the core was performed for the prototype VHTR configuration. The average energy dependent neutron flux obtained for the fuel compacts located in the central region of the core is provided in Fig 9. The flux profile matches expectations for the VHTR system.

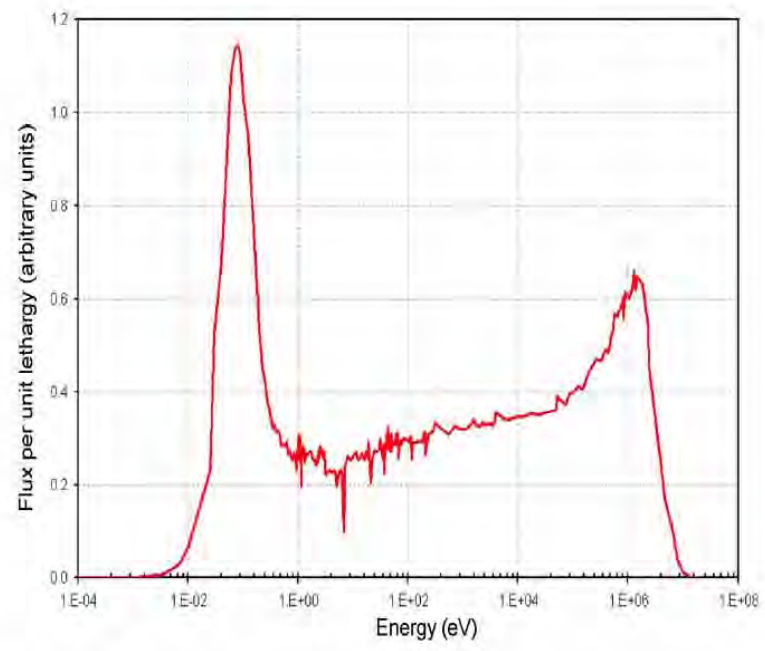

Figure 9. Energy dependent neutron flux in the central fuel compacts.

The VHTR model contains 12 fuel enrichments in the core. Differences in the neutron fluxes are expected in each case. An example is given in Fig. 10, where the averaged neutron fluxes obtained for the fuel compacts containing the lower $(3.3 \%)$ and higher $(9.8 \%)$ enrichments are given. Also noted is the placement of the compacts in the core. The $3.3 \%$ enrichment is located in the central-lower region of the core, and the $9.8 \%$ enrichment being in the upper-outer region of the core. For exact location refer to Table 3 and Fig. 8. 


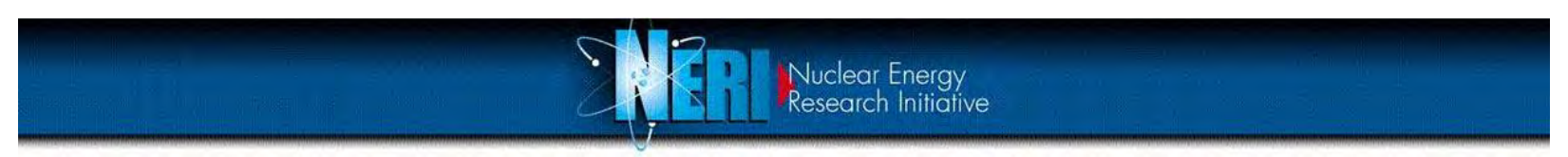

\section{Utilization of MAs as a Fuel Component for Ultra-Long Life VHTR Configurations: Designs, Advantages and Limitations}

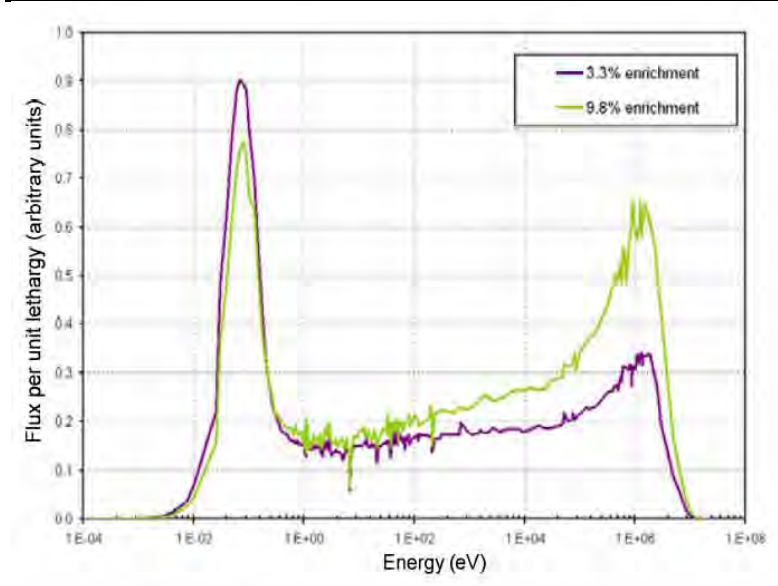

Figure 10. Energy dependent neutron flux in the fuel compacts with different fuel enrichments.

\section{CODE-TO-CODE BENCHMARK}

The HTTR is currently the only operating VHTR prismatic core design, making it a focal point of VHTR related research. The HTTR was designed according to established objectives, which categorize it as a small-scale VHTR. The core size of the HTTR corresponds to about half of that of the future VHTRs [7]. These future reactors will most likely consist of annular core designs, whereas the HTTR is a cylindrical core design.
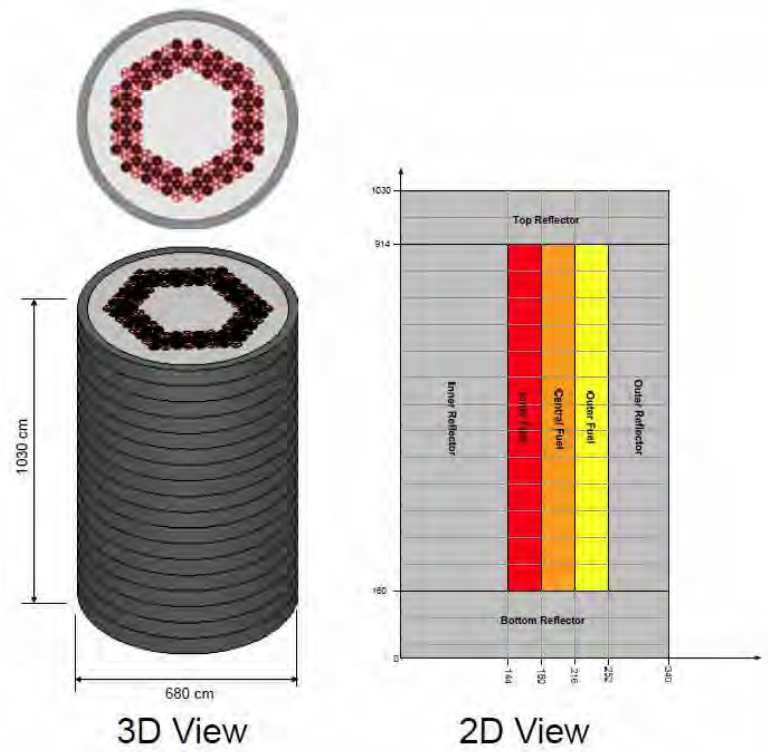

2D View
An annular core is one of the promising core types for the future VHTRs because of its high inherent safety characteristics following a loss of coolant accident. The decay heat removal is enhanced by introduction of the annular core because the heat transfer path will be shortened due to the relatively thin active core region. As a result, the fuel temperature in a loss of coolant accident can be maintained at less than the fuel temperature limit of $1600{ }^{\circ} \mathrm{C}$ [8].

The prismatic whole-core $3 \mathrm{D}$ model was adjusted from the original cylindrical core of the HTTR to that of a larger annular power core $(600 \mathrm{MWt})$. Figure 11 illustrates the geometry changes made to the HTTR in order to construct the larger annular core.

To maintain the consistency of the annular VHTR model an exact model was built in MCNP to perform a code-to-code benchmark for the new configuration. A comparison of the SCALE and MCNP models is provided in Table 6.

Table 6 MCNP vs. SCALE

\begin{tabular}{|l|c|c|}
\hline \multicolumn{1}{|c|}{ Code System } & k-eff & \% difference \\
\hline MCNP & 1.26737 & \multirow{2}{*}{0.124} \\
\hline SCALE & 1.26580 & \\
\hline
\end{tabular}

In addition to the multiplication factor, the average flux within the fuel compacts was also evaluated for the models. As shown in Fig. 12, the flux profiles obtained using both code systems are consistent.

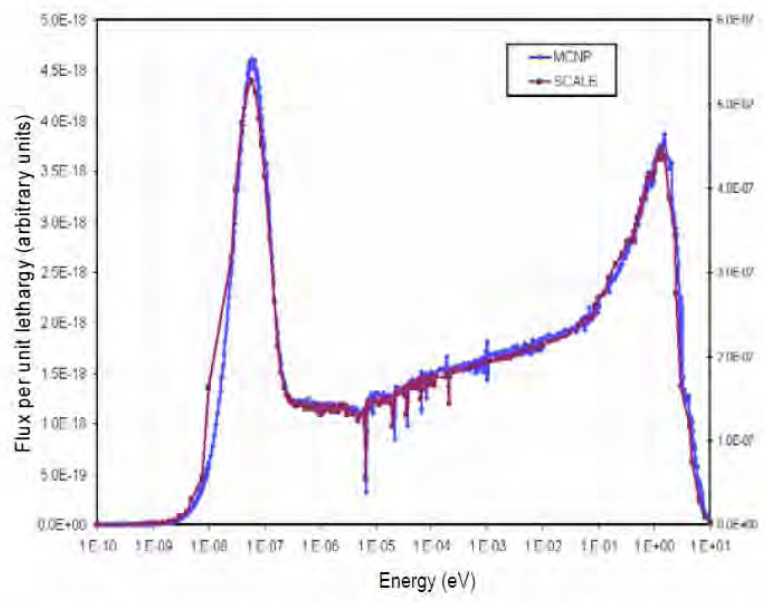

Figure. 12 Energy dependent neutron flux in the fuel compacts - calculations for annular VHTR models using MCNP and SCALE code systems.

Figure 11. VHTR annular power core model configuration. 


\section{Utilization of MAs as a Fuel Component for Ultra-Long Life} VHTR Configurations: Designs, Advantages and Limitations

\section{CONCLUSIONS AND OUTLOOK}

Detailed 3D full-core VHTR models were developed. As an inherent part of the process, the models were validated and verified by performing experiment-to-code and code-to-code benchmarking procedures, which provided substantiation for obtained data and results.

Configuration adjustments were performed on the VHTR cylindrical core benchmark model to create an annular core design. The annular core is a more relevant core layout; to be used in future VHTR systems because it provides superior safety features.

The success of the performed research has provided a seamless transition to upcoming research efforts. The final objective is to assess the possibility, advantages and limitations of achieving ultra-long life VHTRs fueled with TRUs.

The main advantages resulting from TRU fueled VHTRs are their inherent capabilities for utilization of minor actinides from spent LWR fuel, reduction of spent fuel flows, handling per unit of produced energy, and their potential for autonomous operation with minimized maintenance. Consequently, if widely deployed, the developed designs would allow reducing the long-term radiotoxicity and heat load of high-level waste sent to a geologic repository and enable recovery of the energy contained in spent fuel.

This research effort enhances capabilities of the Generation IV VHTR and transforms it to a technology that can deliver electricity, hydrogen, and assist in spent fuel treatment while being inherently safe, environmentally friendly, and proliferation resistant. The technology is also going to be costeffective because of actinide recycling, high burn-up, and reactor lifetime long autonomous operation expanding deployment to developing countries and remote areas.

\section{ACKNOWLEDGMENTS}

This paper is based upon work supported by the U.S Department of Energy under Award Number DE-FC0705ID14655 (05-094).

\section{DISCLAIMER}

This paper was prepared as an account of work sponsored by an agency of the United States Government. Neither the United States Government nor any agency thereof, nor any of their employees, makes any warranty, express or implied, or assumes any legal liability or responsibility for the accuracy, completeness, or usefulness of any information, apparatus. product, or process disclosed, or represents that its use would not infringe privately owned rights.

Reference herein to any specific commercial product process, or service by trade name, trademark, manufacturer, or otherwise does not necessarily constitute or imply its endorsement, recommendation, or favoring by the United States Government or any agency thereof.

The views and opinions of authors expressed herein do not necessarily state or reflect those of the United States Government or any agency thereof.

\section{REFERENCES}

1. "Evaluation of High Temperature Gas-cooled Reactor Performance: Benchmark Analysis Related to Initial Testing of the HTTR and HTR-10", IAEA-TECDOC1382, International Atomic Energy Agency, Vienna, Austria (2003).

2. "SCALE: A Modular Code System for Performing Standardized Computer Analyses for Licensing Evaluation", ORNL/TM-2005/39,Ver.5.1, November 3, 2006, Oak Ridge National Laboratory, Tennessee (2006).

3. David E. Ames II, "Configuration Adjustment Potential of the VHTR Prismatic Cores with Advanced Actinide Fuels", Thesis, Master of Science, August 2006, Nuclear Engineering, Texas A\&M University (2006).

4. Iyoku T., Ueta S., Sumita J., Umeda M., Ishihara M, "Design of Core Components", Nucl. Eng. Des. 233 71-79 (2004).

5. E. E. BENDE, A. H. HOGENBIRK, J. L. KLOOSTERMAN and H. VAN DAM, "Analytical Calculation of the Average Dancoff Factor for a Fuel Kernel in a Pebble-Bed High-Temperature Reactor," Nuc. Sci. Eng. 133, 147-162 (1999).

6. S. FEHER and P.F.A. DE LEEGE, "DANCOFF-MC: A Computer Program for Monte Carlo Calculation of Dancoff Factors in Irregular Geometries," IRI-13 I-95-003 (June 1997).

7. KUNITOMI, K., "Development of New Type of HTGR", (Proc. 73rd JSME Fall Annual Meeting), JSME, Japan, 1995.

8. T. A. TAIWO, T. K. KIM, W. S. YANG, and H. S. KHALIL, "Evaluation of High Temperature Gas-Cooled Reactor Physics Experiments as VHTR Benchmark Problems", ANL-GenIV-059, Argonne National Laboratory, September 2005 


\section{Utilization of MAs as a Fuel Component for Ultra-Long Life}

VHTR Configurations: Designs, Advantages and Limitations

Project 05-094

Final Scientific/Technical Report

\section{UNCERTAINTY ANALYSIS}

A nuclear energy system can be studied as a system that consists of a nuclear reactor (power unit), its associated fuel cycle and other auxiliary infrastructures. Let's assume that performance of this system can fully be described by a generally non-linear vector functional, $\vec{F}_{p}$, of performance characteristics, $\vec{p}$, and design parameters, $\vec{d}$ :

$$
\vec{p}=\vec{F}_{p}(\vec{p}, \vec{d}) .
$$

Evidently, $\vec{p}=\left\{p_{i}, i=1, \ldots, I\right\}$ denotes the set of dependent variables (such as $k_{\text {eff }}, \alpha_{T}, \beta, \alpha, \eta$, other reaction rates and reaction rate ratios of interest and etc.) whereas $\vec{d}=\left\{d_{j}, j=1, \ldots, J\right\}$ denotes the set of independent variables (such as user-defined/selected material properties, operational conditions, system geometry, and etc.). In the present analysis, the non-linear vector functional $\vec{F}_{p}$ is formed by the components of the coupled hybrid Monte Carlo - deterministic model.

For a design parameter $d_{n}, d_{n} \in \vec{d}$, the corresponding rate of change, $\frac{d p_{m}}{d d_{n}}$, in the performance characteristic $p_{m}, p_{m} \in \vec{p}$, due to $d_{n}$ is given by:

$$
\begin{gathered}
\frac{d p_{m}}{d d_{n}}=\sum_{i=1}^{I} \frac{\partial F_{p}^{(m)}}{\partial p_{i}} \frac{d p_{i}}{d d_{n}}+\sum_{j=1}^{J} \frac{\partial F_{p}^{(m)}}{\partial d_{j}} \frac{d d_{j}}{d d_{n}}, \\
\frac{d p_{m}}{d d_{n}}-\left[\frac{\partial F_{p}^{(m)}}{\partial p_{m}} \frac{d p_{m}}{d d_{n}}+\sum_{\substack{i=1 \\
i \neq m}}^{I} \frac{\partial F_{p}^{(m)}}{\partial p_{i}} \frac{d p_{i}}{d d_{n}}\right]=\sum_{j=1}^{J} \frac{\partial F_{p}^{(m)}}{\partial d_{j}} \frac{d d_{j}}{d d_{n}}, \\
\left(1-\frac{\partial F_{p}^{(m)}}{\partial p_{m}}\right) \frac{d p_{m}}{d d_{n}}-\sum_{\substack{i=1 \\
i \neq m}}^{I} \frac{\partial F_{p}^{(m)}}{\partial p_{i}} \frac{d p_{i}}{d d_{n}}=\sum_{j=1}^{J} \frac{\partial F_{p}^{(m)}}{\partial d_{j}} \frac{d d_{j}}{d d_{n}}, \\
\frac{d p_{m}}{d d_{n}}=\frac{1}{1-\frac{\partial F_{p}^{(m)}}{\partial p_{m}}}\left[\sum_{\substack{i=1 \\
i \neq m}}^{I} \frac{\partial F_{p}^{(m)}}{\partial p_{i}} \frac{d p_{i}}{d d_{n}}+\sum_{j=1}^{J} \frac{\partial F_{p}^{(m)}}{\partial d_{j}} \frac{d d_{j}}{d d_{n}}\right] .
\end{gathered}
$$




\section{Utilization of MAs as a Fuel Component for Ultra-Long Life}

VHTR Configurations: Designs, Advantages and Limitations

Project 05-094

Final Scientific/Technical Report

A dimensionless sensitivity indicator, $S_{m, n}$, is often defined as the relative perturbation in the performance characteristic $p_{m}$ due to the specific relative perturbation in the selected design parameter $d_{n}$ of the model. If the finite perturbations of $p_{m}$ and $d_{n}$ are denoted as $\delta_{p}^{(m)}$ and $\delta_{d}^{(n)}$, respectively, then the sensitivity indicator $S_{m, n}$ can be expressed as following:

$$
S_{m, n} \cong \frac{\left(\frac{\delta_{p}^{(m)}}{p_{m}}\right)}{\left(\frac{\delta_{d}^{(n)}}{d_{n}}\right)}=\frac{d_{n}}{p_{m}}\left(\frac{\delta_{p}^{(m)}}{\delta_{d}^{(n)}}\right) .
$$

Assuming small perturbations of $p_{m}$ and $d_{n}$, the finite values of $\delta_{p}^{(m)}$ and $\delta_{d}^{(n)}$ can be replaced by differentials:

$$
\begin{gathered}
\delta_{p}^{(m)} \rightarrow \partial p_{m}, \\
\delta_{d}^{(n)} \rightarrow \partial d_{n},
\end{gathered}
$$

$$
S_{m, n}=\left.\frac{d_{n}}{p_{m}} \frac{\partial p_{m}}{\partial d_{n}}\right|_{p_{m}, d_{n}} .
$$

Therefore, the dimensionless sensitivity indicator, $S_{m, n}$, can be calculated using the specific values of $p_{m}$ and $d_{n}$ and the corresponding partial derivative of $p_{m}$ with respect to $d_{n}$ at point $d_{n}$. Using the above-derived expression for $\frac{d p_{m}}{d d_{n}}$ instead of $\frac{\partial p_{m}}{\partial d_{n}}, S_{m, n}$ becomes:

$$
S_{m, n}=\frac{d_{n}}{p_{m}} \frac{1}{1-\frac{\partial F_{p}^{(m)}}{\partial p_{m}}}\left[\sum_{\substack{i=1 \\ i \neq m}}^{I} \frac{\partial F_{p}^{(m)}}{\partial p_{i}} \frac{d p_{i}}{d d_{n}}+\sum_{j=1}^{J} \frac{\partial F_{p}^{(m)}}{\partial d_{j}} \frac{d d_{j}}{d d_{n}}\right] .
$$

Accounting for the complete sets of performance characteristics, $\left\{p_{m}, m=1, \ldots, M\right\}$, and design parameters, $\left\{d_{n}, n=1, \ldots, N\right\}, S_{m, n}$ form a $M \times N$ dimensionless sensitivity matrix $\mathbb{S}$. Analysis of the matrix $\mathbb{S}$ yields evaluations of an overall modeling reliability with identification of a computational design envelop expected for the VHTR configurations with MAs. If further generalized, this methodology will be suitable for multi-parametric design optimization. For the considered HTTR and HTR-10 configurations, Table 3 summarizes the dimensionless sensitivity 
indicators , $S_{m, n}=S_{k, T}$, for variations of $k_{\text {eff }}$ due to variations of the isothermal core temperature, $T_{\text {core }}$ :

$$
S_{m, n}=S_{k, T} \cong \frac{T_{\text {core }}}{k_{\text {eff }}}\left(\frac{\delta_{k}}{\delta_{T}}\right)
$$

Table 3. Sensitivity of the VHTR Criticality to Isothermal Core Temperature

\begin{tabular}{|c|c|c|c|c|c|c|c|c|c|}
\hline \multicolumn{5}{|c|}{$\begin{array}{c}\text { HTTR } \\
\text { (Control Rods at Critical Position Determined at 300K) }\end{array}$} & \multicolumn{5}{|c|}{$\begin{array}{c}\text { HTR-10 } \\
\text { (Control Rods Withdrawn, Homogeneous Core) }\end{array}$} \\
\hline $\begin{array}{l}T_{\text {core }} \\
\text { (K) }\end{array}$ & $k_{e f f}$ & $\delta_{k}$ & $\delta_{T}$ & $\begin{array}{l}S_{k, T} \\
(\%)\end{array}$ & $\begin{array}{l}T_{\text {core }} \\
\text { (K) }\end{array}$ & $k_{e f f}$ & $\delta_{k}$ & $\delta_{T}$ & $\begin{array}{l}S_{k, T} \\
(\%)\end{array}$ \\
\hline 300.0 & 1.001 & -0.0042 & 40.0 & -3.1 & 300.0 & 1.153 & -0.0180 & 93.0 & -5.0 \\
\hline 340.0 & 0.997 & -0.0028 & 40.0 & -2.4 & 393.0 & 1.135 & -0.0100 & 80.0 & -4.3 \\
\hline 380.0 & 0.994 & -0.0121 & 40.0 & $\begin{array}{l}-11.6 \\
\end{array}$ & \multirow[t]{3}{*}{473.0} & \multirow[t]{3}{*}{1.125} & \multirow[t]{3}{*}{-0.0107} & \multirow[t]{3}{*}{50.0} & \multirow[t]{3}{*}{-9.0} \\
\hline 420.0 & 0.982 & -0.0068 & 40.0 & $\begin{array}{l}-7.3 \\
\end{array}$ & & & & & \\
\hline 460.0 & 0.975 & -0.0027 & 20.0 & -6.4 & & & & & \\
\hline
\end{tabular}

The developed formalism provides the basis for the chosen uncertainty analysis methodology and for the selection of appropriate tools. The uncertainty evaluation is divided into two sequential parts:

1) Sensitivity analysis,

2) Uncertainty analysis.

The first part, sensitivity analysis, is approached as a way to determine the importance of different parameters and components of the model (design parameters, $\left\{d_{n}, n=1, \ldots, N\right\}$ ) on the output of the model (performance characteristics, $\left\{p_{m}, m=1, \ldots, M\right\}$ ). The second part, uncertainty analysis, is viewed as a direct follow-up based on the results of the first task. The variance of $\left\{p_{m}, m=1, \ldots, M\right\}$ is assumed to be the measure of uncertainty and is treated as an output variable that is related to the input variables through the model. It describes how the parameter estimate would vary in repeated sampling.

In the present analysis, the strategy consists of several tasks that result in the following multi-step approach: 
- Preliminary analysis of the VHTRs with MAs;

- Identification of the relevant sets of performance characteristics, $\left\{p_{m}, m=1, \ldots, M\right\}$, and design parameters, $\left\{d_{n}, n=1, \ldots, N\right\}$;

- Sensitivity analysis on $\left\{p_{m}, m=1, \ldots, M\right\}$ with respect to $\left\{d_{n}, n=1, \ldots, N\right\}$;

- Analysis of uncertainty effects due to $\left\{d_{n}, n=1, \ldots, N\right\}$;

- Reliability analysis of the VHTR modeling with identification of the computational design envelop expected for the VHTR configurations with MAs.

As emphasized by the last step, the uncertainty (sensitivity) analysis is used to determine the choice of modeling reliability requirements:

- Specification of the required accuracies for the VHTR characteristics including properties to be calculated using the nuclear data;

- Uncertainty partitioning between the contributing nuclear data;

- Uncertainty partitioning between the VHTR parameters;

- Overall modeling reliability evaluations with identification of the computational design envelop expected for the VHTR configurations with MAs.

Accuracy requirement is assumed to be inversely proportional to the sensitivity due to the existing uncertainty effects.

There are existing computer code systems for dealing with uncertainties that are critical to the design of nuclear energy systems. New deterministic and stochastic methods are available for simulation, optimization, and synthesis of advanced nuclear energy systems. Many of these methods have originally been developed for applications outside nuclear technology. Uncertainty analysis, as applied in the ecological, medical, and general risk analysis areas, covers a wide range of techniques. They range from simple descriptive procedures to quantitative estimation of uncertainty, to more formal decision-based procedures. In particular, in designing complex engineering systems, multidisciplinary design optimization has become a systematic approach to optimization of complex, coupled engineering systems, where "multidisciplinary" refers to the different aspects that must be included in designing a system that involves multiple interacting disciplines.

The chosen uncertainty analysis methodology links the applied suite of neutronics and thermal hydraulics codes and the generalized codes for universal sensitivity analysis, calibration and uncertainty evaluations in a framework. Because this approach requires parameter distributions and special sampling techniques to be applied for calculations and analysis of 
performance characteristics, the sensitivity/uncertainty codes form a computational shell over the neutronics/thermal-hydraulics codes.

As demonstrated by the preceding discussion, there are obvious discrepancies among ENDF/B-6.8, JENDL-3.3 and JEF-2.2. These discrepancies contribute to the existing uncertainties and limit reliability of the analysis by causing differences in reactor physics characteristics. Uncertainty effects due to nuclear data are taken into consideration and are analyzed using the SCALE 5.0/TSUNAMI sensitivity and uncertainty analysis methodology. Dimensionless sensitivity indicators (or sensitivity coefficients as defined in the SCALE manual), $S_{m, n}=S_{k, \Sigma(\vec{r})}$, produced by the TSUNAMI computational sequences predict the relative changes in $k_{\text {eff }}$ due to relative changes (small perturbations) in the neutron macroscopic crosssection, $\Sigma(\vec{r})$, of the transport operator at some point $\vec{r}$ in phase space:

$$
\begin{gathered}
S_{m, n}=S_{k, \Sigma(\vec{r})} \cong \frac{\Sigma(\vec{r})}{k_{e f f}}\left(\frac{\delta_{k}}{\delta_{\Sigma(\vec{r})}}\right), \\
S_{k, \Sigma(\vec{r})}=\frac{\Sigma(\vec{r})}{k_{\text {eff }}} \frac{\partial k_{e f f}}{\partial \Sigma(\vec{r})} .
\end{gathered}
$$

In the present analysis, the generalized codes for universal sensitivity analysis, calibration and uncertainty evaluations are represented by UCODE-2005 code system and the associated six post-processors. These codes are used with the applied suite of neutronics and thermal hydraulics codes to perform sensitivity analysis; data needs assessment, calibration, prediction, and uncertainty analysis. Statistics are calculated to quantify the comparison of observations and simulated equivalents, including a weighted least-squares objective function. Parameters are estimated using nonlinear regression: a weighted least-squares objective function is minimized with respect to the parameter values using a modified Gauss-Newton method or a double-dogleg technique.

\subsection{Uncertainty Effects due to Nuclear Data}

\section{Minor Actinides}

The long-term potential radiotoxicity of spent nuclear fuel is associated mainly with the actinide elements particularly the transuranium nuclides (TRU $=\mathrm{Pu}, \mathrm{Np}, \mathrm{Am}, \mathrm{Cm}, \ldots$ ). As emphasized above, currently some of the recovered plutonium is recycled in the form of uranium-plutonium mixed-oxide (MOX) fuel in LWRs, the remaining mix of minor actinides 
(MA: neptunium (Np), americium (Am), curium (Cm) and higher-Z actinides) and fission products is conditioned for final waste disposal.

Over a very long time period (hundred thousand years), these constitute a significant radiological source term within a spent fuel repository. With increasing burnup, the generation of MAs becomes more and more important.

Although the intrinsic insolubility of actinides in deep geological formations contributes to the effective isolation of TRUs, if the public and/or political acceptance of very long term disposal of HLW could not be obtained, the removal of MAs from HLW would be a technical solution which might reduce the residual radiotoxicity of the HLW.

The closed fuel cycle with partitioning and transmutation (P\&T) is the most comprehensive approach. The absence of the fission products and the MAs in spent fuel will reduce the stowaway time to about 1000 years for the fuel waste to attain the emission level of natural uranium.

Reactor designs with the MA-bearing fuel compounds require more accurate nuclear data for TRUs. The data of main interest are fission and capture cross-sections, fission neutron yields, fission neutron spectra and scattering cross sections.

Due to current research interests and needs of many national programs including U.S. DOE AFCI and Generation IV programs, the status of nuclear data for MAs has been extensively evaluated in many studies including this project. In many independent assessments it was concluded that the currently available experimental data are insufficient to produce the accurate evaluated data for MAs and new measurements are needed. The experimental post-irradiation benchmark studies indicated that the current JENDL, ENDF/B-VI and JEF yield significant discrepancies in the predicted amounts of MAs and multiplication factors.

\section{Analysis of the Available Nuclear Data for MAs}

In the present study, the evaluated data given in ENDF/B-6.8, JENDL-3.3, and JEF-2.2 for ${ }^{235} \mathrm{U},{ }^{238} \mathrm{U},{ }^{237} \mathrm{~Np},{ }^{241} \mathrm{Am},{ }^{242 \mathrm{~m}} \mathrm{Am},{ }^{243} \mathrm{Am},{ }^{242} \mathrm{Cm},{ }^{243} \mathrm{Cm},{ }^{244} \mathrm{Cm}$, and ${ }^{245} \mathrm{Cm}$ are compared. Uranium isotopes, ${ }^{235} \mathrm{U}$ and ${ }^{238} \mathrm{U}$, are included as reference nuclides. The pointwise interpolated data have been directly reconstructed using the evaluated data files without spectral weighting. For all nuclides, the fission $\left(\sigma_{f}, \mathrm{MT}=18\right)$, capture $\left(\sigma_{c}, \mathrm{MT}=102\right)$, total neutron yield per fission $\left(v_{f}, \mathrm{MT}=452\right)$ and the corresponding delayed neutron yield per fission ( $\left.v_{d}, \mathrm{MT}=455\right)$ are taken into consideration. The available data were analyzed for neutron energies between $1.0 \mathrm{E}-5 \mathrm{eV}$ and $20 \mathrm{MeV}$ assuming a linear interpolation.

Not all these data are available in all libraries considered. Table 4 shows data availability for MAs. Grid cells marked with " $x$ ” represent available data. 


\section{S 1 mp \\ Research Initiative}

Utilization of MAs as a Fuel Component for Ultra-Long Life VHTR Configurations: Designs, Advantages and Limitations

Project 05-094

Final Scientific/Technical Report

Table 4. Availability of the MA Nuclear Data in ENDF/B-6.8, JENDL-3.3, and JEF-2.2

\begin{tabular}{|c|c|c|c|c|c|c|c|c|c|c|}
\hline File and Data Set & $\begin{array}{c}\text { Reference } \\
\text { Nuclides }\end{array}$ & \multicolumn{10}{c|}{ Minor Actinides } \\
\cline { 2 - 9 } & ${ }^{235} \mathrm{U}$ & ${ }^{238} \mathrm{U}$ & ${ }^{237} \mathrm{~Np}$ & ${ }^{241} \mathrm{Am}$ & ${ }^{242 m} \mathrm{Am}$ & ${ }^{243} \mathrm{Am}$ & ${ }^{242} \mathrm{Cm}$ & ${ }^{243} \mathrm{Cm}$ & ${ }^{244} \mathrm{Cm}$ & ${ }^{245} \mathrm{Cm}$ \\
\hline
\end{tabular}

ENDF/B-6.8

\begin{tabular}{|c|c|c|c|c|c|c|c|c|c|c|}
\hline МТ18 & $\mathrm{X}$ & $\mathrm{X}$ & $\mathrm{X}$ & $\mathrm{X}$ & $\mathrm{X}$ & $\mathrm{X}$ & $\mathrm{X}$ & $\mathrm{X}$ & $\mathrm{X}$ & $\mathrm{X}$ \\
\hline MT102 & $\mathrm{X}$ & $\mathrm{X}$ & $\mathrm{X}$ & $\mathrm{X}$ & $\mathrm{X}$ & $\mathrm{X}$ & $\mathrm{X}$ & $\mathrm{X}$ & $\mathrm{X}$ & $\mathrm{X}$ \\
\hline MT452 & $\mathrm{X}$ & $\mathrm{X}$ & $\mathrm{X}$ & $\mathrm{X}$ & $\mathrm{X}$ & $\mathrm{X}$ & $\mathrm{X}$ & $\mathrm{X}$ & $\mathrm{X}$ & $\mathrm{X}$ \\
\hline MT455 & $\mathrm{X}$ & $\mathrm{X}$ & $\mathrm{X}$ & $\mathrm{X}$ & $\mathrm{X}$ & $\mathrm{X}$ & $\mathrm{X}$ & $\mathrm{X}$ & $\mathrm{X}$ & $\mathrm{X}$ \\
\hline
\end{tabular}

JENDL-3.3

\begin{tabular}{|c|c|c|c|c|c|c|c|c|c|c|}
\hline MT18 & $X$ & $\mathrm{X}$ & $X$ & $\mathrm{X}$ & $\mathrm{X}$ & $X$ & $\mathrm{X}$ & $\mathrm{X}$ & $\mathrm{X}$ & $\mathrm{X}$ \\
\hline MT102 & $X$ & $X$ & $X$ & $X$ & $X$ & $X$ & $X$ & $X$ & $X$ & $X$ \\
\hline MT452 & $X$ & $X$ & $X$ & $\mathrm{X}$ & $\mathrm{X}$ & $X$ & $X$ & $X$ & $X$ & $X$ \\
\hline MT455 & $\mathrm{X}$ & $\mathrm{X}$ & $\mathrm{X}$ & $\mathrm{X}$ & $X$ & $X$ & $X$ & $\mathrm{X}$ & - & $\mathrm{X}$ \\
\hline
\end{tabular}

JEF-2.2

\begin{tabular}{|l|c|c|c|c|c|c|c|c|c|c|}
\hline MT18 & $\mathrm{x}$ & $\mathrm{x}$ & $\mathrm{x}$ & $\mathrm{x}$ & $\mathrm{x}$ & $\mathrm{x}$ & $\mathrm{x}$ & $\mathrm{x}$ & $\mathrm{x}$ & $\mathrm{x}$ \\
\hline MT102 & $\mathrm{x}$ & $\mathrm{x}$ & $\mathrm{x}$ & $\mathrm{x}$ & $\mathrm{x}$ & $\mathrm{x}$ & $\mathrm{x}$ & $\mathrm{x}$ & $\mathrm{x}$ & $\mathrm{x}$ \\
\hline MT452 & $\mathrm{x}$ & $\mathrm{x}$ & $\mathrm{x}$ & - & - & $\mathrm{x}$ & - & - & - & - \\
\hline MT455 & $\mathrm{x}$ & $\mathrm{x}$ & $\mathrm{x}$ & - & - & - & - & - & - & - \\
\hline
\end{tabular}

Figure 1 shows microscopic fission cross sections of MAs. Pointwise energy dependence is reconstructed using the nuclear data derived from ENDF/B-6.8, JENDL-3.3, and JEF-2.2, respectively. It can be seen that ${ }^{242 \mathrm{~m}} \mathrm{Am}$ and ${ }^{245} \mathrm{Cm}$ have larger fission cross-sections than ${ }^{235} \mathrm{U}$ in the thermal energy region up till neutron energies of about $0.2 \mathrm{eV}$. 


\section{STn \\ Research Initiative}

Utilization of MAs as a Fuel Component for Ultra-Long Life

VHTR Configurations: Designs, Advantages and Limitations

Project 05-094

Final Scientific/Technical Report

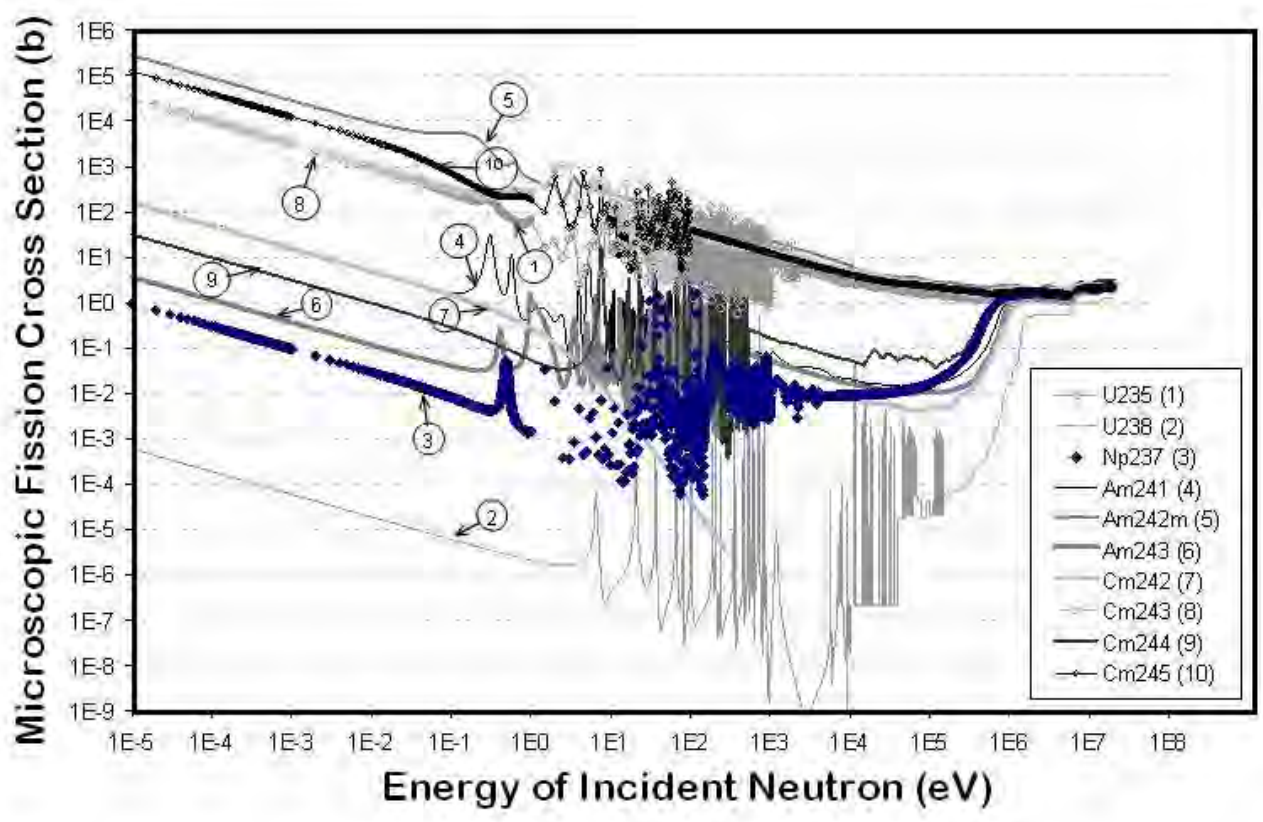

(a) ENDF/B-6.8.

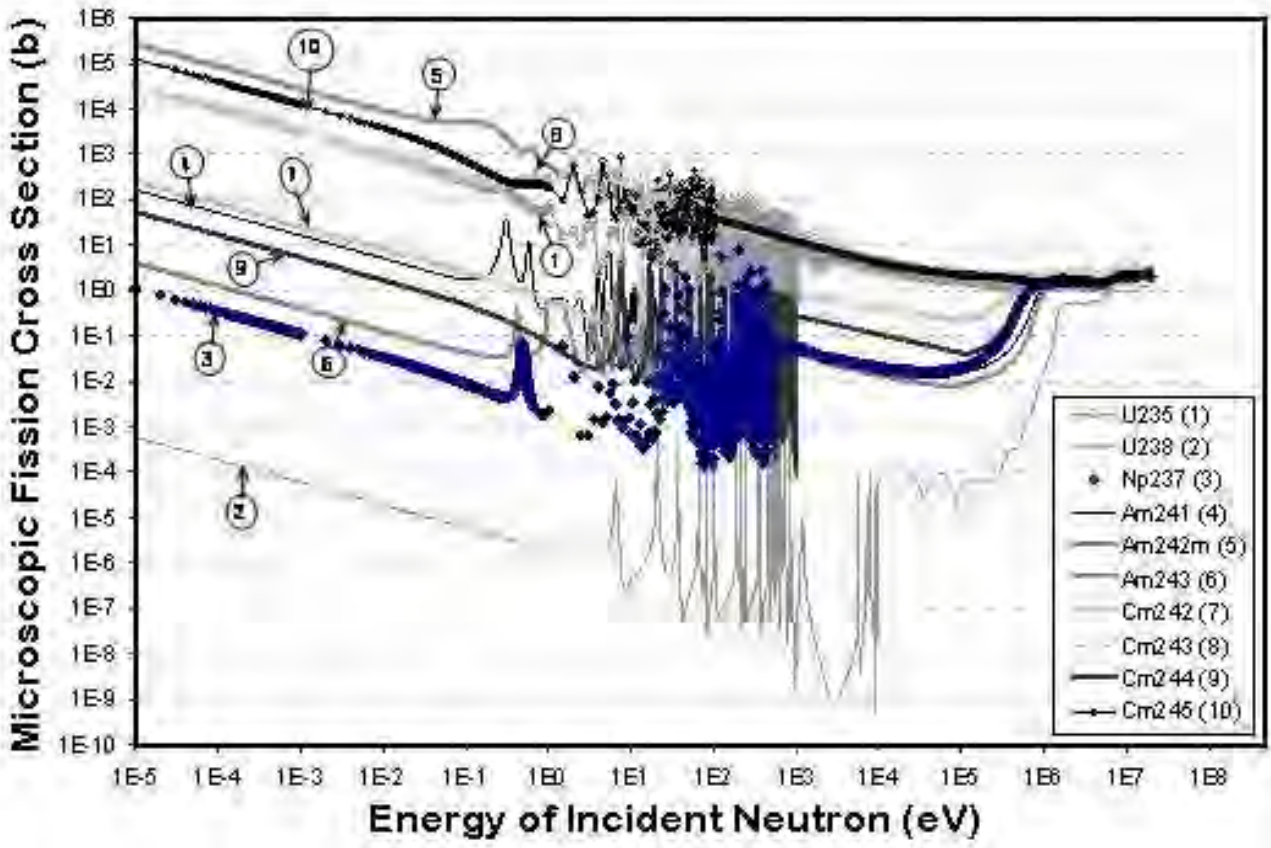

(b) JENDL-3.3. 


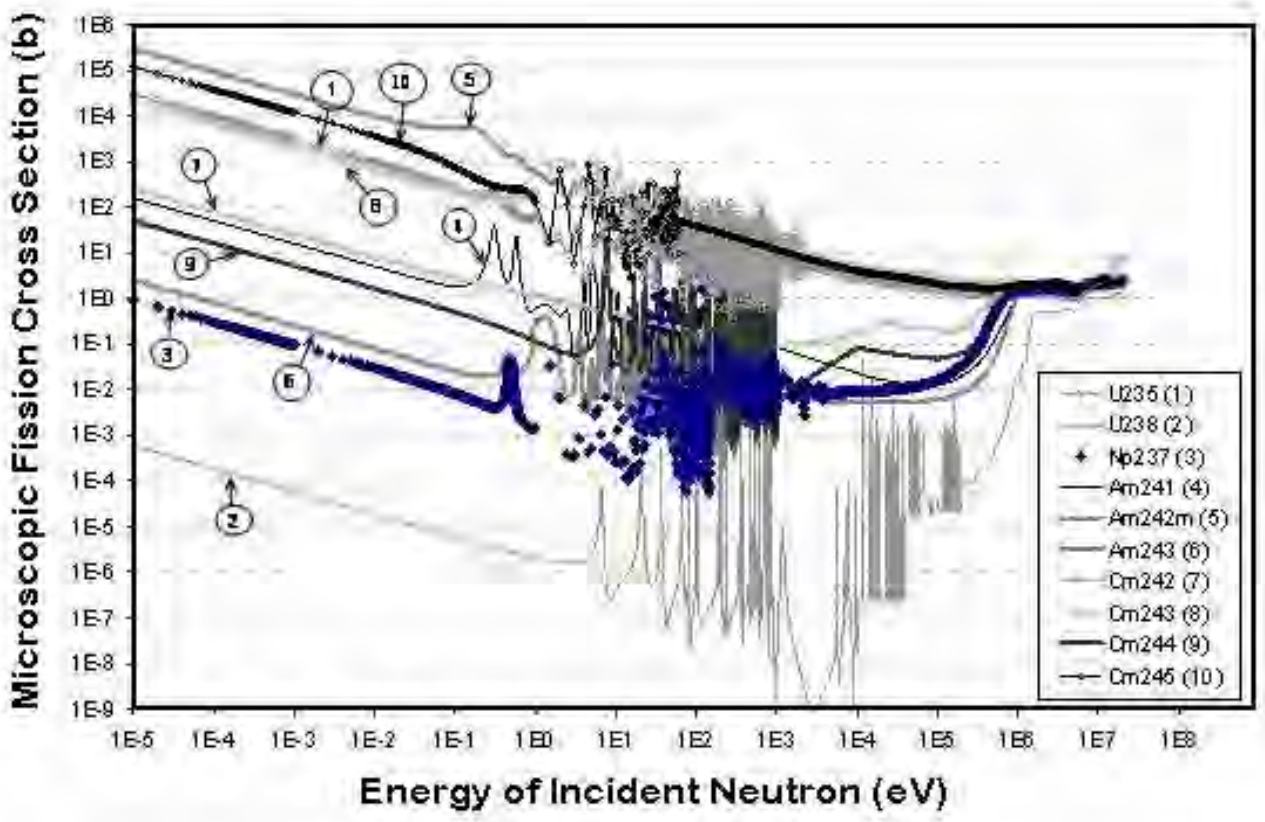

(c) JEF-2.2.

Figure 1. Microscopic fission cross sections of MAs in ENDF/B-6.8, JENDL-3.3, JEF-2.2.

According to Figure 1, fission cross sections of ${ }^{235} \mathrm{U}$ and ${ }^{243} \mathrm{Cm}$ are also similar within the thermal energy region up till neutron energies of about $0.2 \mathrm{eV}$. Furthermore, all MAs generally have higher fission cross-section than ${ }^{238} \mathrm{U}$.

Table 5 shows the ranking of the microscopic fission cross sections based on the order of magnitude. Using the magnitude ranking as an assessment metric, it can be seen that the overall sequence of the magnitudes of fission cross-sections is consistent between the nuclear data files. Observed consistency between the evaluated data files doesn't mean that the data are accurate. Most probably, the same experimental data have been used as the basis for cross sections in different files.

Table 5. Ranking of the Microscopic Fission Cross Sections up to $0.2 \mathrm{eV}^{*}$

\begin{tabular}{|l|c|c|c|c|c|c|c|c|c|c|}
\hline File & ${ }^{235} \mathbf{U}$ & ${ }^{238} \mathbf{U}$ & ${ }^{237} \mathbf{N p}$ & ${ }^{241} \mathbf{A m}$ & ${ }^{242 m} \mathbf{A m}$ & ${ }^{243} \mathbf{A m}$ & ${ }^{242} \mathbf{C m}$ & ${ }^{243} \mathbf{C m}$ & ${ }^{244} \mathbf{C m}$ & ${ }^{245} \mathbf{C m}$ \\
\hline ENDF/B-6.8 & 3 & 10 & 9 & 6 & 1 & 8 & 5 & 4 & 7 & 2 \\
\hline JENDL-3.3 & 3 & 10 & 9 & 6 & 1 & 8 & 5 & 4 & 7 & 2 \\
\hline JEF-2.2 & 3 & 10 & 9 & 6 & 1 & 8 & 5 & 4 & 7 & 2 \\
\hline
\end{tabular}

*Cross section ranking basis: 1 - largest cross section value, 10 - smallest cross section value. 


\section{T/2 \\ Research Initiative}

Utilization of MAs as a Fuel Component for Ultra-Long Life

VHTR Configurations: Designs, Advantages and Limitations

Project 05-094

Final Scientific/Technical Report

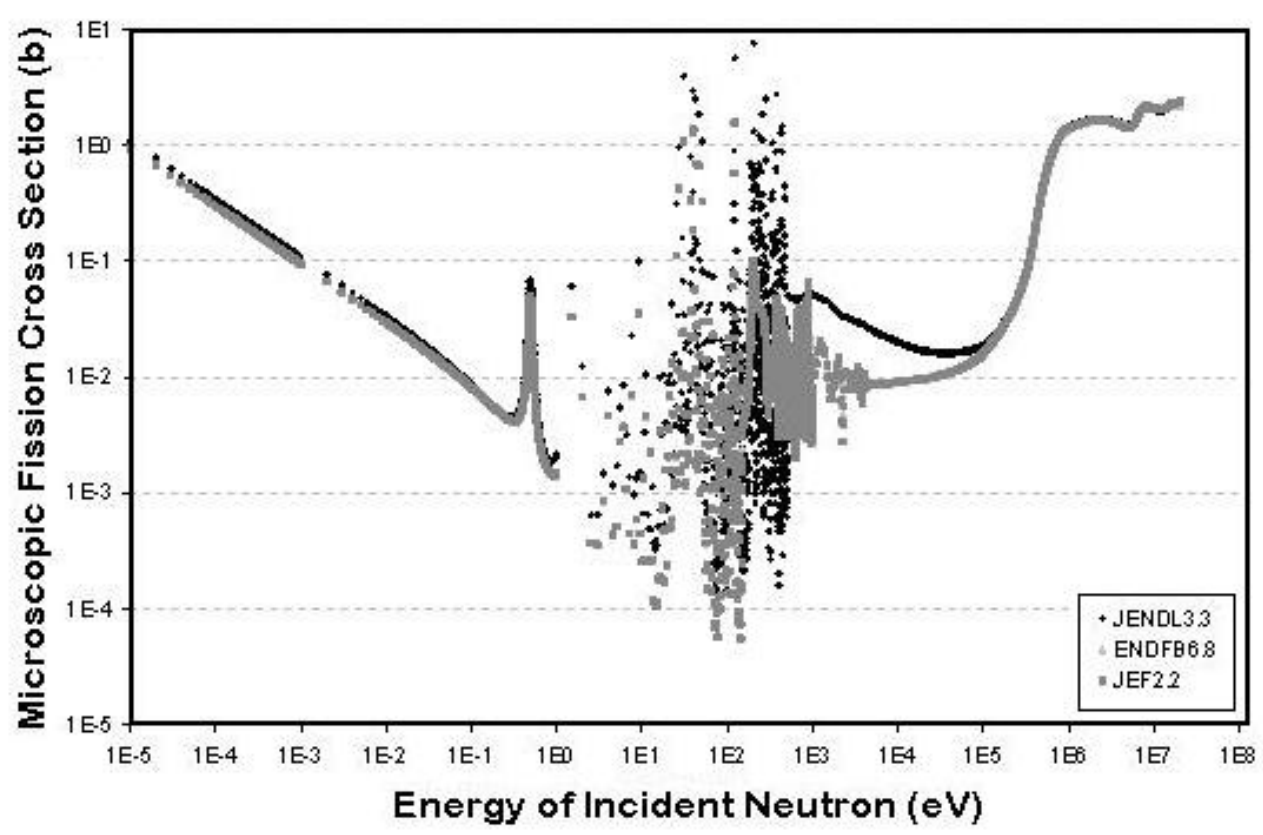

(a) ${ }^{237} \mathrm{~Np}$.

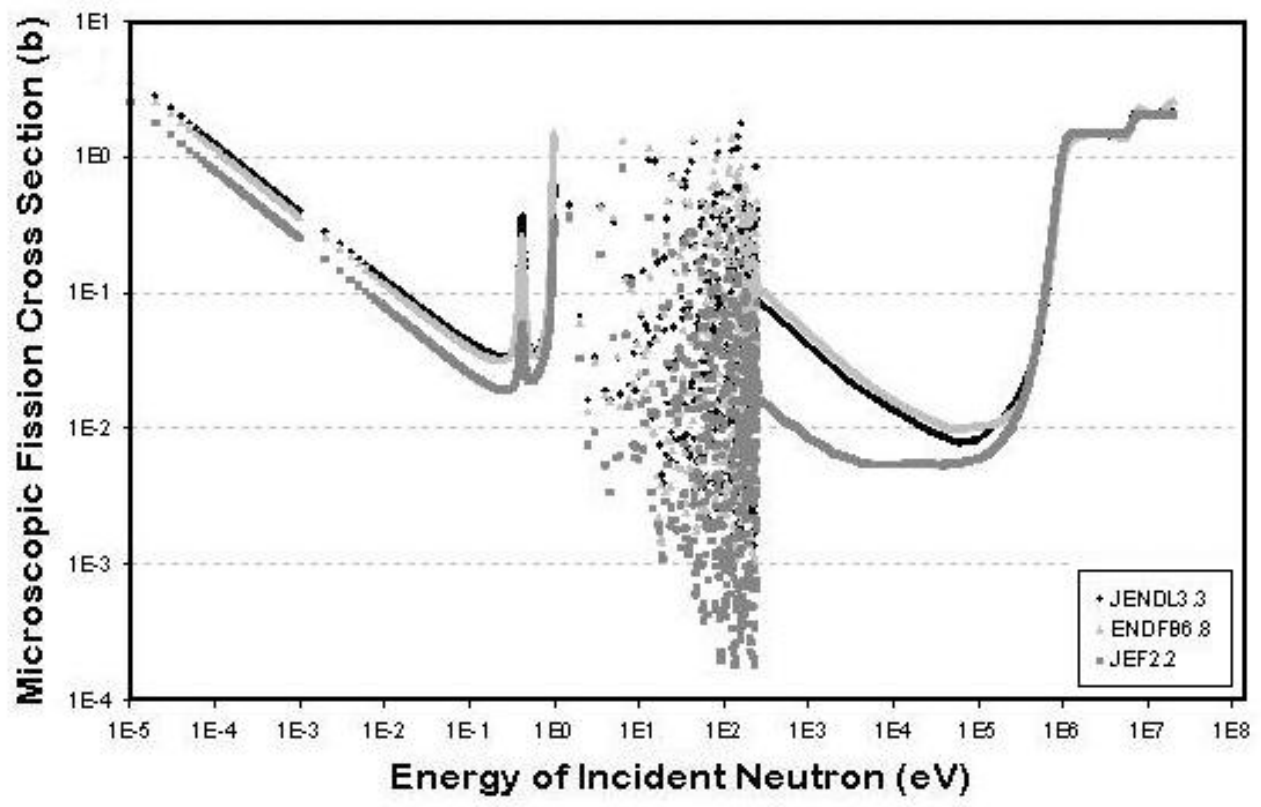

(b) ${ }^{243} \mathrm{Am}$. 
Utilization of MAs as a Fuel Component for Ultra-Long Life

VHTR Configurations: Designs, Advantages and Limitations

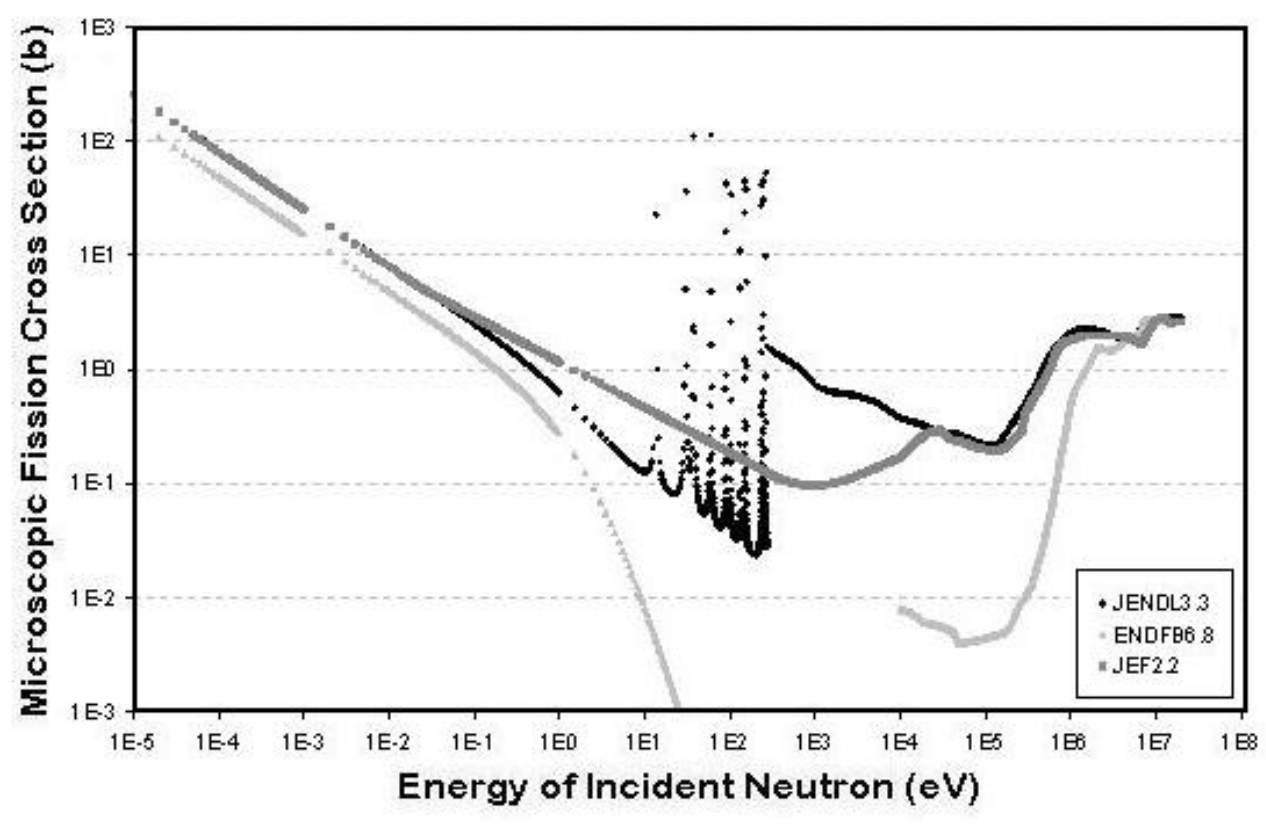

(c) ${ }^{242} \mathrm{Cm}$.

Figure 2. Microscopic fission cross sections of ${ }^{237} \mathrm{~Np},{ }^{243} \mathrm{Am}$ and ${ }^{242} \mathrm{Cm}$.

To illustrate differences between the evaluated nuclear data in ENDF/B-6.8, JENDL-3.3 and JEF-2.2, Figure 2 shows the evaluated microscopic fission cross sections of ${ }^{237} \mathrm{~Np}$, ${ }^{243} \mathrm{Am}$, and ${ }^{242} \mathrm{Cm}$. Despite the common trends observed in Figure 1, Figure 2 shows that the data for particular nuclides may vary significantly.

For example, as shown in Figure 2, the evaluated fission cross-section data of ${ }^{242} \mathrm{Cm}$ in ENDF/B-6.8 have a discontinuity between $275 \mathrm{eV}$ and 1.0E+4 eV. On the other hand, the fission cross-section data of ${ }^{242} \mathrm{Cm}$ in JEF 2.2 appear to be a continuous function. Conversely, the fission cross-section data of ${ }^{242} \mathrm{Cm}$ in JENDL-3.3 have a well-defined resolved resonance structure within the same energy interval between $275 \mathrm{eV}$ and $1.0 \mathrm{E}+4 \mathrm{eV}$. Overall, the nuclear data of ENDF/B-6.8 are largely underestimated whereas JENDL-3.3 and JEF-2.2 reproduce well experimental data.

Similar to fission cross sections, Figure 3 shows microscopic capture cross sections of MAs. Pointwise energy dependence is reconstructed using the nuclear data derived from ENDF/B-6.8, JENDL-3.3, and JEF-2.2, respectively. It can be seen that ${ }^{237} \mathrm{~Np},{ }^{241} \mathrm{Am},{ }^{242 \mathrm{~m}} \mathrm{Am}$ and ${ }^{245} \mathrm{Cm}$ have larger capture cross sections than ${ }^{235} \mathrm{U}$ in the thermal energy region up till neutron energies of about $0.1 \mathrm{eV}$. Same as for fission cross sections of MAs, Figure 3 demonstrates that all MAs generally have higher capture cross-section than ${ }^{238} \mathrm{U}$. 


\section{ST:

Utilization of MAs as a Fuel Component for Ultra-Long Life

VHTR Configurations: Designs, Advantages and Limitations

Project 05-094

Final Scientific/Technical Report

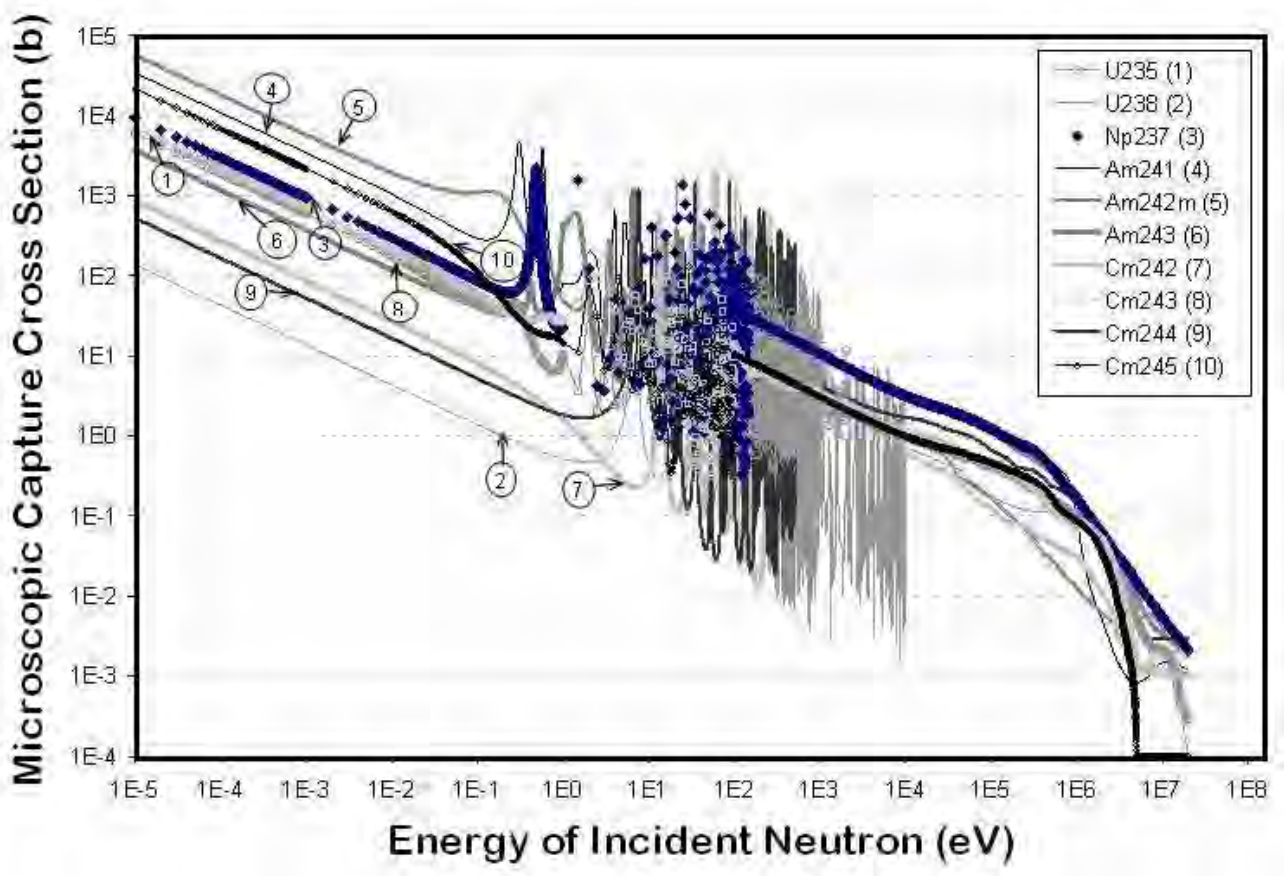

(a) ENDF/B-6.8.

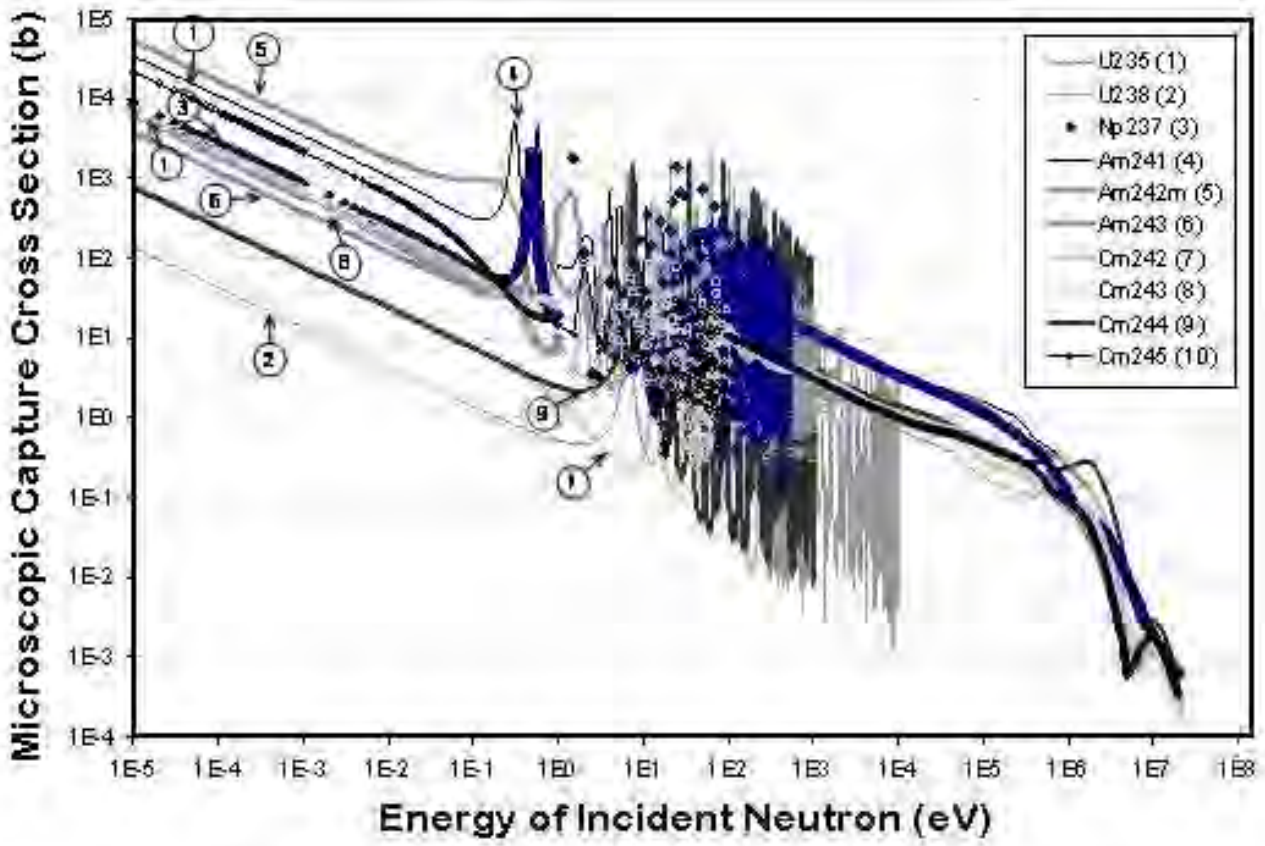

(b) JENDL-3.3. 


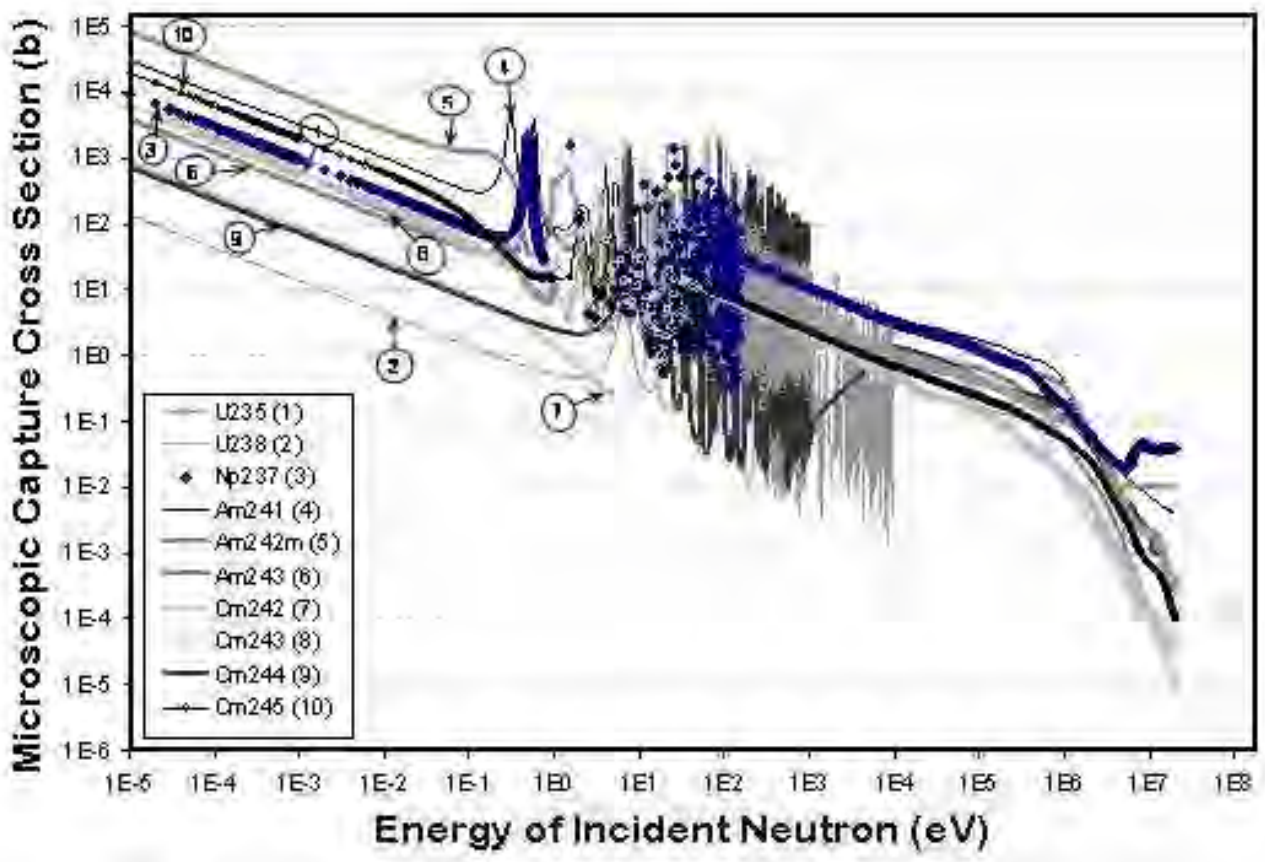

(c) JEF-2.2.

Figure 3. Microscopic capture cross sections of MAs in ENDF/B-6.8, JENDL-3.3, JEF-2.2.

Following the same approach as for Table 5, Table 6 shows the ranking of the microscopic capture cross sections based on the order of magnitude. Using the magnitude ranking as an assessment metric, it can be seen that the overall sequence of the magnitudes of capture crosssections is consistent between nuclear data files. As before, this consistency doesn't mean, of course, that the data are accurate. The same experimental data could have been used as the basis for cross-sections in different files.

Table 6. Ranking of the Microscopic Capture Cross Sections up to $0.1 \mathrm{eV}^{*}$

\begin{tabular}{|l|c|c|c|c|c|c|c|c|c|c|}
\hline File & ${ }^{235} \mathbf{U}$ & ${ }^{238} \mathbf{U}$ & ${ }^{237} \mathbf{N p}$ & ${ }^{241} \mathbf{A m}$ & ${ }^{242 m} \mathbf{A m}$ & ${ }^{243} \mathbf{A m}$ & ${ }^{242} \mathbf{C m}$ & ${ }^{243} \mathbf{C m}$ & ${ }^{24} \mathbf{C m}$ & ${ }^{245} \mathbf{C m}$ \\
\hline ENDF/B-6.8 & 6 & 10 & 4 & 2 & 1 & 7 & 8 & 5 & 9 & 3 \\
\hline JENDL-3.3 & 6 & 10 & 4 & 2 & 1 & 7 & 8 & 5 & 9 & 3 \\
\hline JEF-2.2 & 6 & 10 & 4 & 2 & 1 & 7 & 8 & 5 & 9 & 3 \\
\hline
\end{tabular}

*Cross section ranking basis: 1 - largest cross section value, 10 - smallest cross section value. 
Figure 4 illustrates the existing differences between the evaluated capture cross section data in ENDF/B-6.8, JENDL-3.3, and JEF-2.2. The microscopic capture cross section of ${ }^{242} \mathrm{Cm}$ is chosen as an example. As can be seen, the capture cross section data of ${ }^{242} \mathrm{Cm}$ in these files are consistent for thermal and resonance energy ranges. However, there are significant differences in the available nuclear data for fast energy range. The JENDL-3.3 fast energy data disagree with the data in ENDF/B-6.8 and JEF-2.2, which have lesser variations.

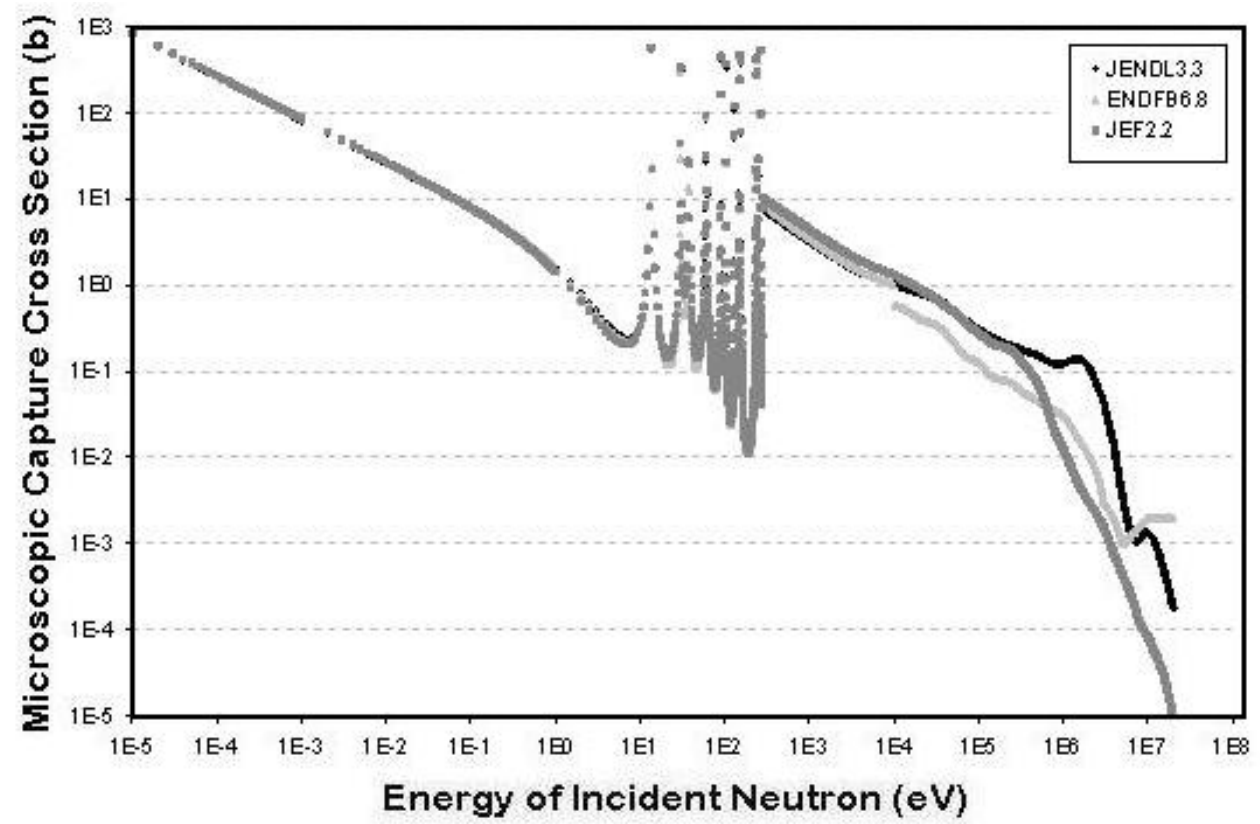

Figure 4. Microscopic capture cross section of ${ }^{242} \mathrm{Cm}$.

Figure 5 shows total neutron yields per fission of MAs. It can be noted that MAs have larger neutron yields than uranium isotopes.

Unlike the previously examined microscopic fission and capture cross sections, there is no consistency between nuclear data files. Using ${ }^{237} \mathrm{~Np}$ and ${ }^{243} \mathrm{Am}$ as examples, the existing differences between the total neutron yield data in ENDF/B-6.8 and JENDL-3.3 are illustrated in Figure 6.

To improve data representation and to facilitate enhanced fitting to experimental values, JENDL-3.3 adopted a fitting curve. However, the measured data points stored in EXFOR are not reproduced with the fitting curve because they were not available at the time of evaluation. 
Utilization of MAs as a Fuel Component for Ultra-Long Life

VHTR Configurations: Designs, Advantages and Limitations

Final Scientific/Technical Report

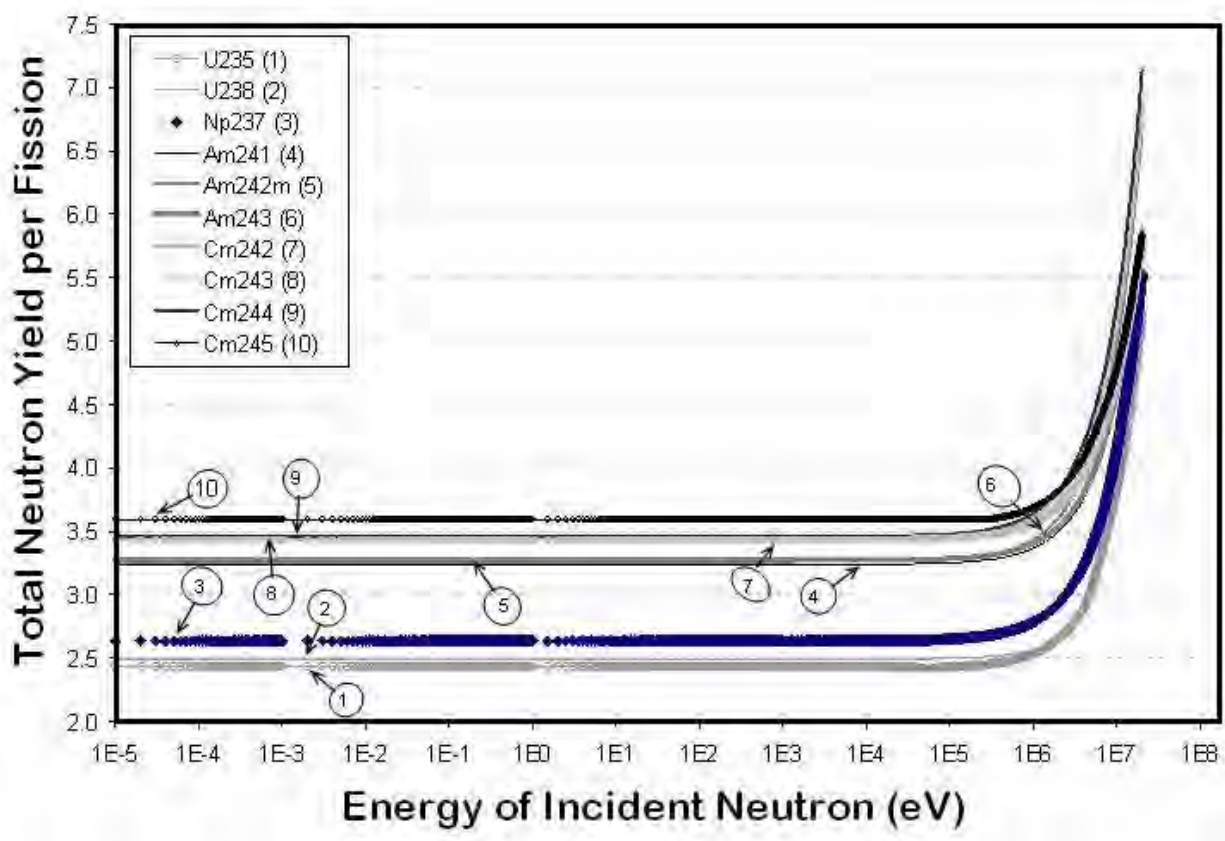

(a) ENDF/B-6.8.

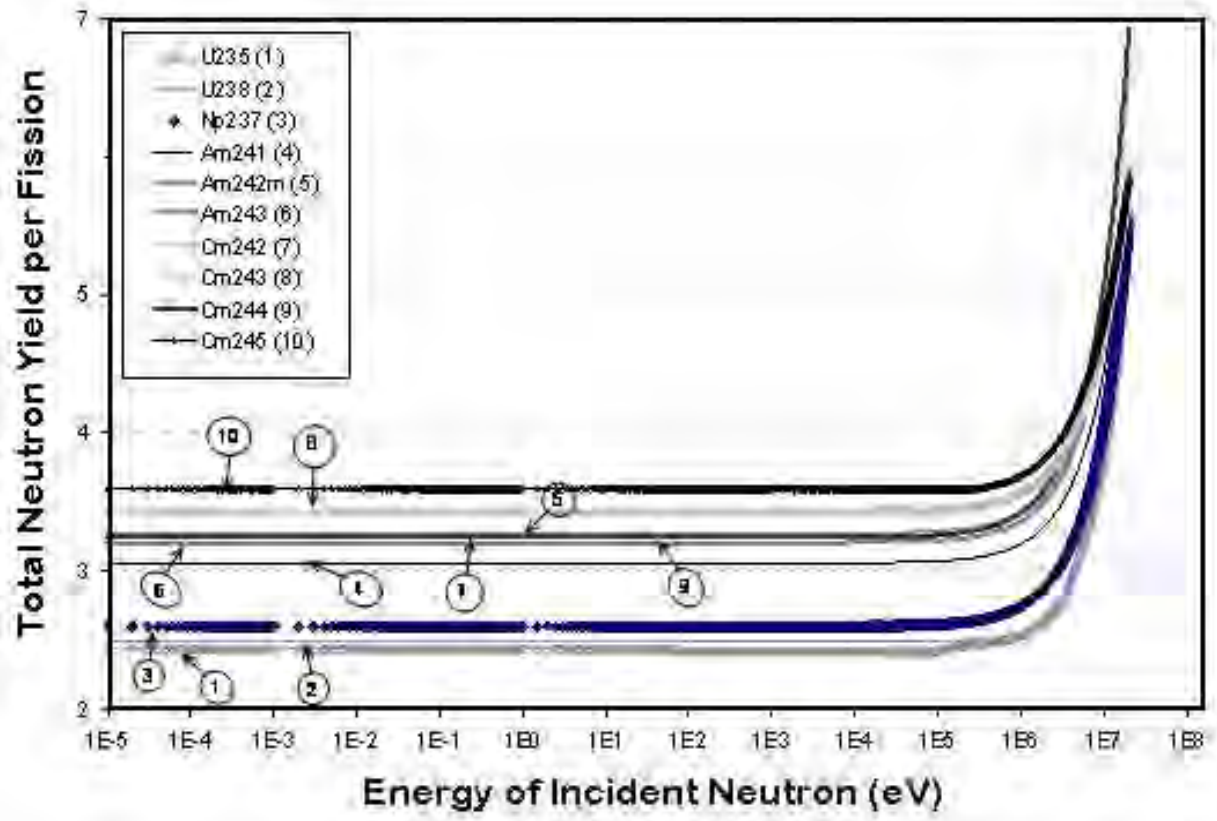

(b) JENDL-3.3.

Figure 5. Total neutron yields per fission of MAs in ENDF/B-6.8, JENDL-3.3. 
Utilization of MAs as a Fuel Component for Ultra-Long Life

VHTR Configurations: Designs, Advantages and Limitations

Final Scientific/Technical Report

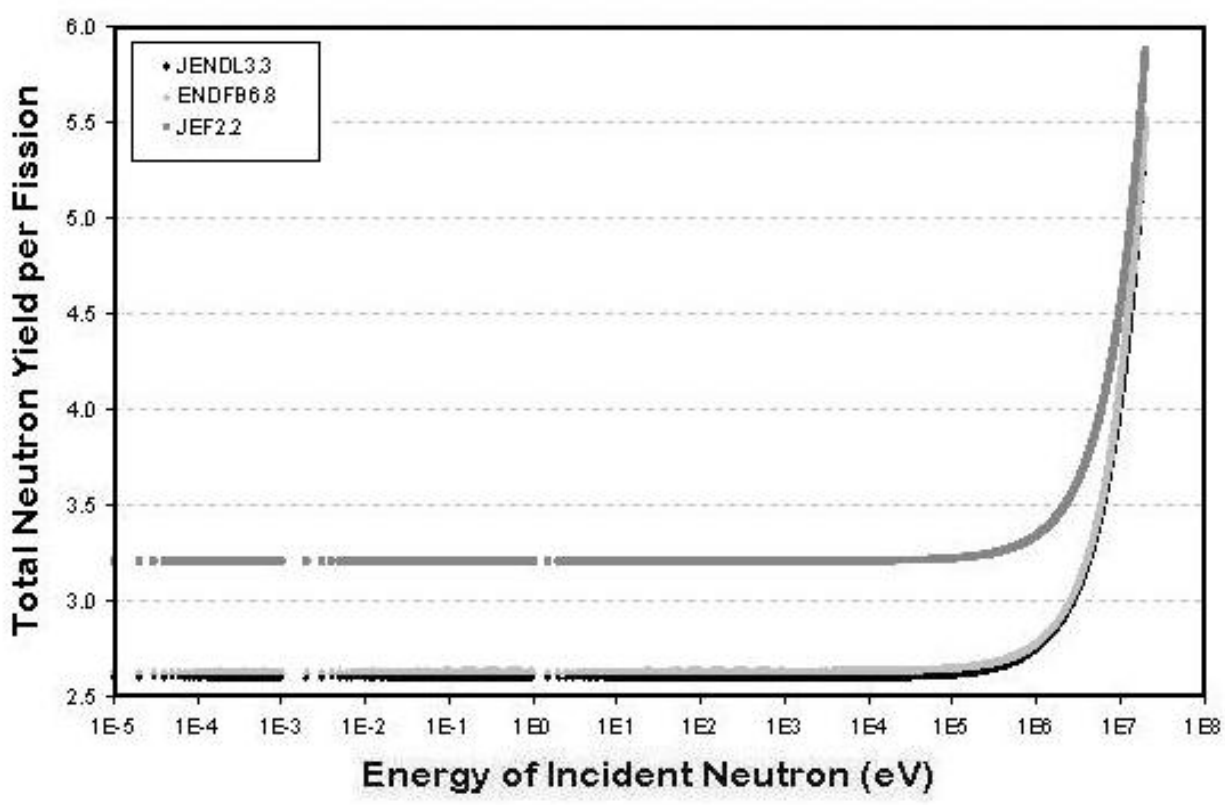

(a) ${ }^{237} \mathrm{~Np}$.

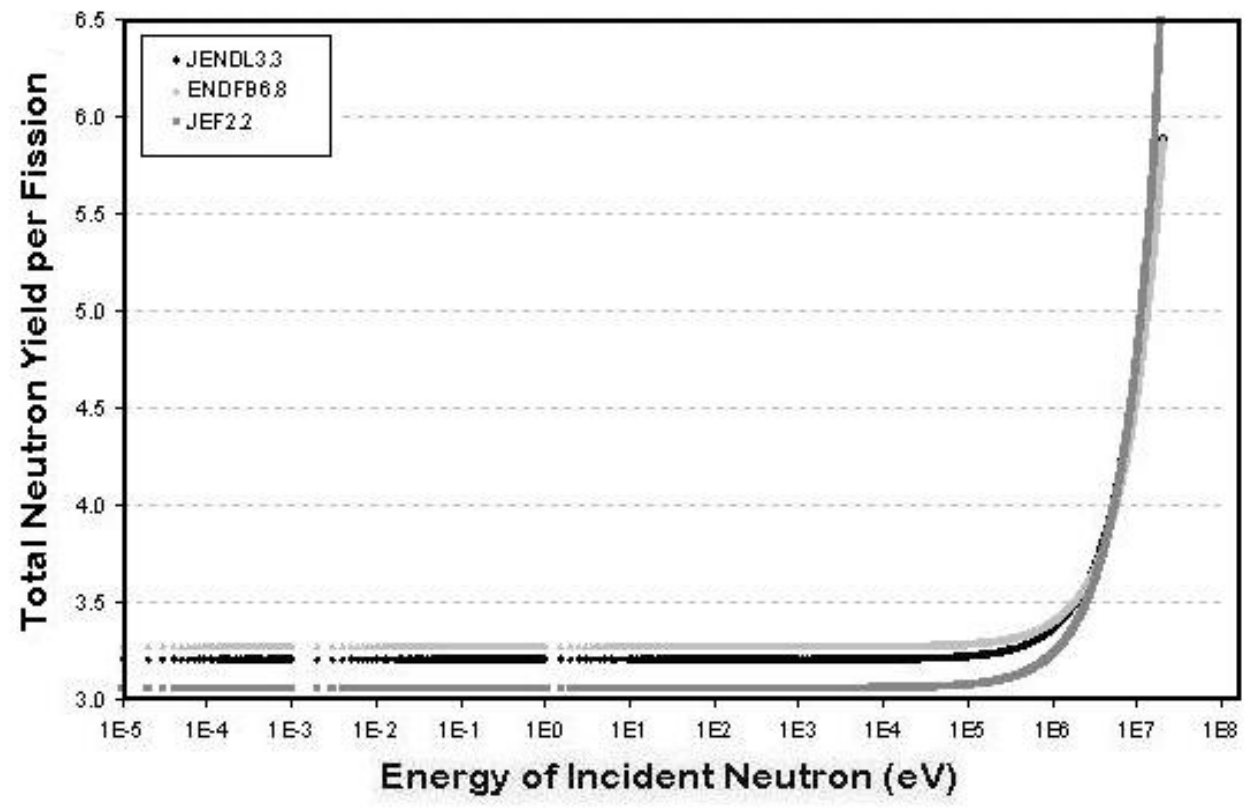

(b) ${ }^{243} \mathrm{Am}$.

Figure 6. Total neutron yields per fission of ${ }^{237} \mathrm{~Np}$ and ${ }^{243} \mathrm{Am}$. 
Utilization of MAs as a Fuel Component for Ultra-Long Life VHTR Configurations: Designs, Advantages and Limitations

Project 05-094

Final Scientific/Technical Report

Table 7 shows the ranking of the total neutron yields per fission based on the order of magnitude. Using the magnitude ranking as an assessment metric, the existing discrepancies can be clearly shown.

Table 7. Ranking of the Total Neutron Yields per Fission up to $1.0 \mathrm{E}+5 \mathrm{eV}^{*}$

\begin{tabular}{|l|c|c|c|c|c|c|c|c|c|c|}
\hline File & ${ }^{235} \mathbf{U}$ & ${ }^{238} \mathbf{U}$ & ${ }^{237} \mathbf{N p}$ & ${ }^{241} \mathbf{A m}$ & ${ }^{242 m} \mathbf{A m}$ & ${ }^{243} \mathbf{A m}$ & ${ }^{24} \mathbf{C m}$ & ${ }^{243} \mathbf{C m}$ & ${ }^{244} \mathbf{C m}$ & ${ }^{245} \mathbf{C m}$ \\
\hline ENDF/B-6.8 & 10 & 9 & 8 & 7 & 6 & 5 & 3 & 4 & 2 & 1 \\
\hline JENDL-3.3 & 10 & 9 & 8 & 7 & 3 & 6 & 4 & 2 & 5 & 1 \\
\hline JEF-2.2 & 4 & 3 & 1 & - & - & 2 & - & - & - & - \\
\hline
\end{tabular}

*Ranking basis: 1 - largest value, 10 - smallest value.

Based on the existing data differences in ENDF/B-6.8, JENDL-3.3 and JEF-2.2 as demonstrated in Figure 2, Figure 4, and Figure 6, the need for new experimental evaluations is apparent. For many nuclides, the current experimental data are not enough and too old. Accurate and reliable nuclear data for TRUs are of paramount importance for the design, safety, and performance analysis of VHTRs with MA-bearing fuels.

\section{Basic Benchmark Analysis of the MA Data Using Elementary Reaction Rate Ratios}

As illustrated by the preceding discussion, there are significant discrepancies found among ENDF/B-6.8, JENDL-3.3 and JEF-2.2 with respect to the nuclear data for MAs. These discrepancies are expected to cause differences in reactor physics characteristics. To facilitate the present studies, the basic benchmark analysis is performed using elementary reaction rate ratios:

- Capture-to-fission rate ratio for a single nuclide $X, \alpha^{X}\left(E_{n}\right)$ :

$$
\alpha^{X}\left(E_{n}\right)=\frac{\sigma_{c}^{X}\left(E_{n}\right)}{\sigma_{f}^{X}\left(E_{n}\right)},
$$

where: $\sigma_{f}^{X}\left(E_{n}\right)$ is the microscopic fission cross section of nuclide $X$ (MT=18);

$\sigma_{c}^{X}\left(E_{n}\right)$ is the microscopic capture cross section of nuclide $X$ $(\mathrm{MT}=102)$.

- Delayed neutron fraction, $\beta^{X}\left(E_{n}\right)$ :

$$
\beta^{X}\left(E_{n}\right)=\frac{v_{d}^{X}\left(E_{n}\right)}{v_{f}^{X}\left(E_{n}\right)},
$$




\section{Utilization of MAs as a Fuel Component for Ultra-Long Life}

VHTR Configurations: Designs, Advantages and Limitations

where: $\quad v_{f}^{X}\left(E_{n}\right)$ is the total (prompt plus delayed) neutron yield per fission caused by a neutron ${ }_{0}^{1} n$ with energy $E_{n}(\mathrm{eV})(\mathrm{MT}=452)$;

$v_{d}^{X}\left(E_{n}\right)$ is the delayed neutron yield per fission caused by a neutron ${ }_{0}^{1} n$ with energy $E_{n}(\mathrm{eV})(\mathrm{MT}=455)$.

- Number of neutrons released per neutron absorbed in a single nuclide $X, \eta^{X}\left(E_{n}\right)$ :

$$
\eta^{X}\left(E_{n}\right)=\frac{v_{f}^{X}\left(E_{n}\right) \sigma_{f}^{X}\left(E_{n}\right)}{\sigma_{a}^{X}\left(E_{n}\right)}=\frac{v_{f}^{X}\left(E_{n}\right)}{1+\alpha^{X}\left(E_{n}\right)},
$$

where: $\quad v_{f}^{X}\left(E_{n}\right)$ is the total (prompt plus delayed) neutron yield per fission caused by a neutron ${ }_{0}^{1} n$ with energy $E_{n}(\mathrm{eV})(\mathrm{MT}=452)$;

$\sigma_{f}^{X}\left(E_{n}\right)$ is the microscopic fission cross section of nuclide $X$ (MT=18);

$\sigma_{a}^{X}\left(E_{n}\right)$ is the microscopic absorption cross section of nuclide $X$ computed as a sum of $\sigma_{f}^{X}\left(E_{n}\right) \quad(\mathrm{MT}=18)$ and $\sigma_{c}^{X}\left(E_{n}\right)$ $(\mathrm{MT}=102)$.

The relative balance between the probability of fission and radiative capture is an extremely important factor in nuclear reactor applications. This balance is characterized by the capture-tofission rate ratio, $\alpha^{X}\left(E_{n}\right)$. The elementary reaction rate ratio $\eta^{X}\left(E_{n}\right)$ characterizes relative capabilities to sustain a fission chain reaction.

The number of neutrons released per neutron absorbed is shown in Figure 7. It can be seen that ${ }^{242 \mathrm{~m}} \mathrm{Am},{ }^{243} \mathrm{Cm}$ and ${ }^{245} \mathrm{Cm}$ have the largest values of $\eta^{X}\left(E_{n}\right)$ for thermal and resonance energies of incident neutrons. Table 8 shows the ranking of $\eta^{X}\left(E_{n}\right)$ based on the order of magnitude. Using the magnitude ranking as an assessment metric, the existing discrepancies can be clearly shown. As illustrated in Figure 8, despite the ranking consistency, $\eta^{X}\left(E_{n}\right)$ may vary significantly.

Table 8. Ranking of the Number of Neutrons Released per Thermal Neutron Absorbed*

\begin{tabular}{|l|c|c|c|c|c|c|c|c|c|c|}
\hline File & ${ }^{235} \mathbf{U}$ & ${ }^{238} \mathbf{U}$ & ${ }^{237} \mathbf{N p}$ & ${ }^{241} \mathbf{A m}$ & ${ }^{242 m} \mathbf{A m}$ & ${ }^{243} \mathbf{A m}$ & ${ }^{242} \mathbf{C m}$ & ${ }^{243} \mathbf{C m}$ & ${ }^{244} \mathbf{C m}$ & ${ }^{245} \mathbf{C m}$ \\
\hline ENDF/B-6.8 & 4 & 10 & 9 & 7 & 3 & 8 & 5 & 2 & 6 & 1 \\
\hline JENDL-3.3 & 4 & 10 & 9 & 7 & 3 & 8 & 5 & 2 & 6 & 1 \\
\hline JEF-2.2 & 1 & 4 & 3 & - & - & 2 & - & - & - & - \\
\hline
\end{tabular}

*Ranking basis: 1 - largest value, 10 - smallest value. 


\section{STh \\ Research Initiative}

Utilization of MAs as a Fuel Component for Ultra-Long Life

VHTR Configurations: Designs, Advantages and Limitations

Project 05-094

Final Scientific/Technical Report

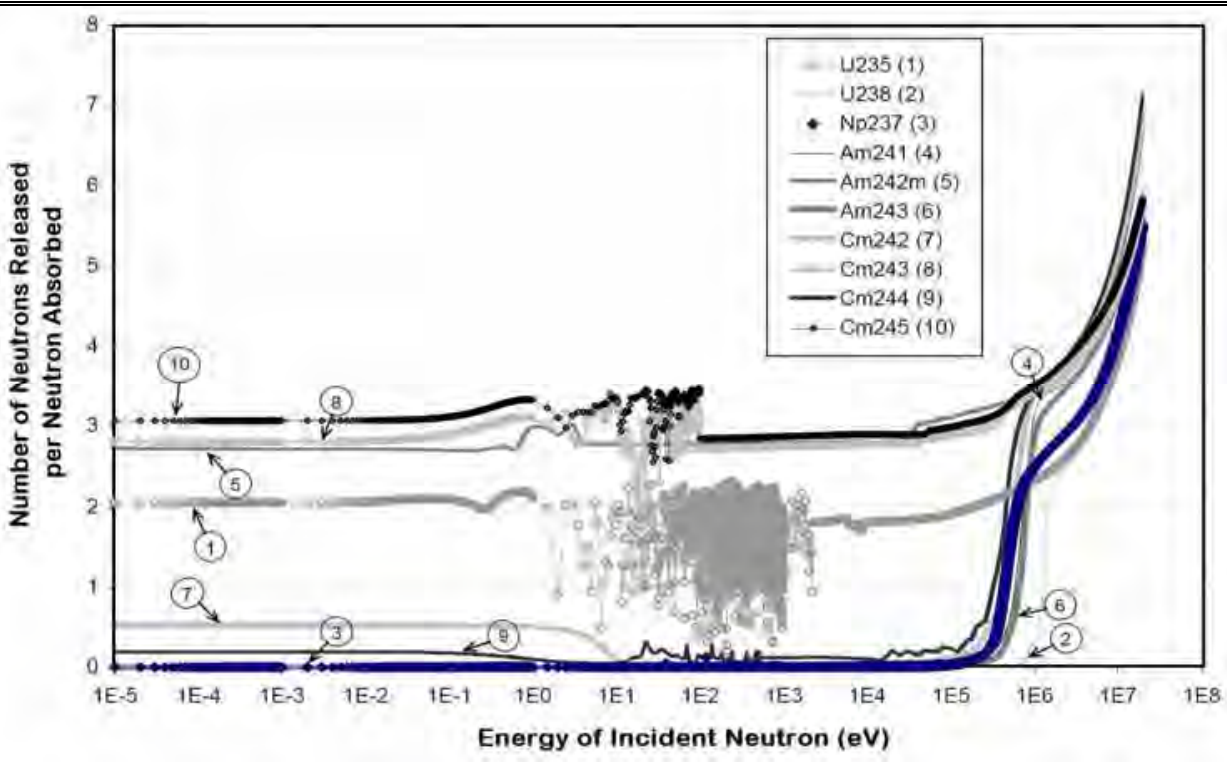

(a) ENDF/B-6.8.

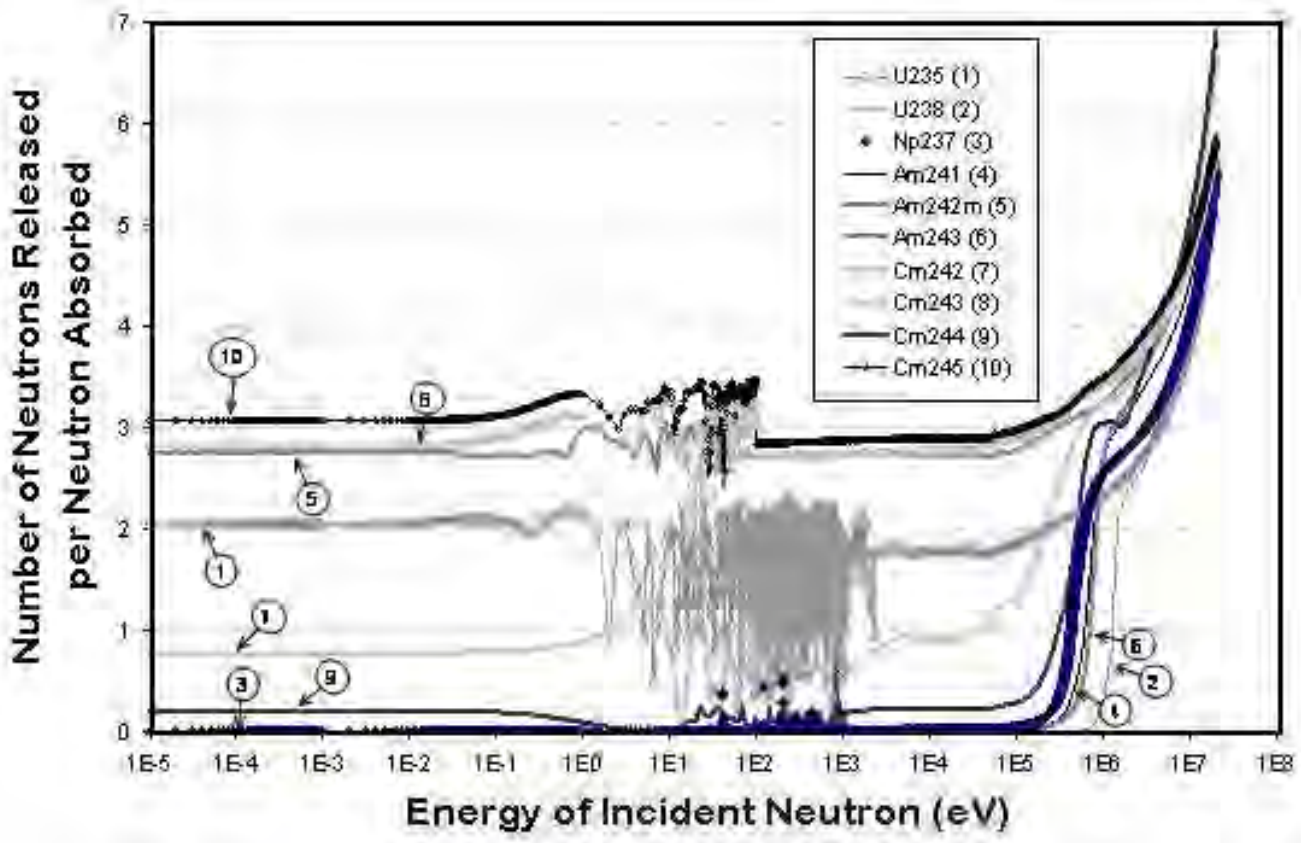

(b) JENDL-3.3.

Figure 7. Number of neutrons released per neutron absorbed (ENDF/B-6.8, JENDL-3.3). 
Utilization of MAs as a Fuel Component for Ultra-Long Life

VHTR Configurations: Designs, Advantages and Limitations

Project 05-094

Final Scientific/Technical Report

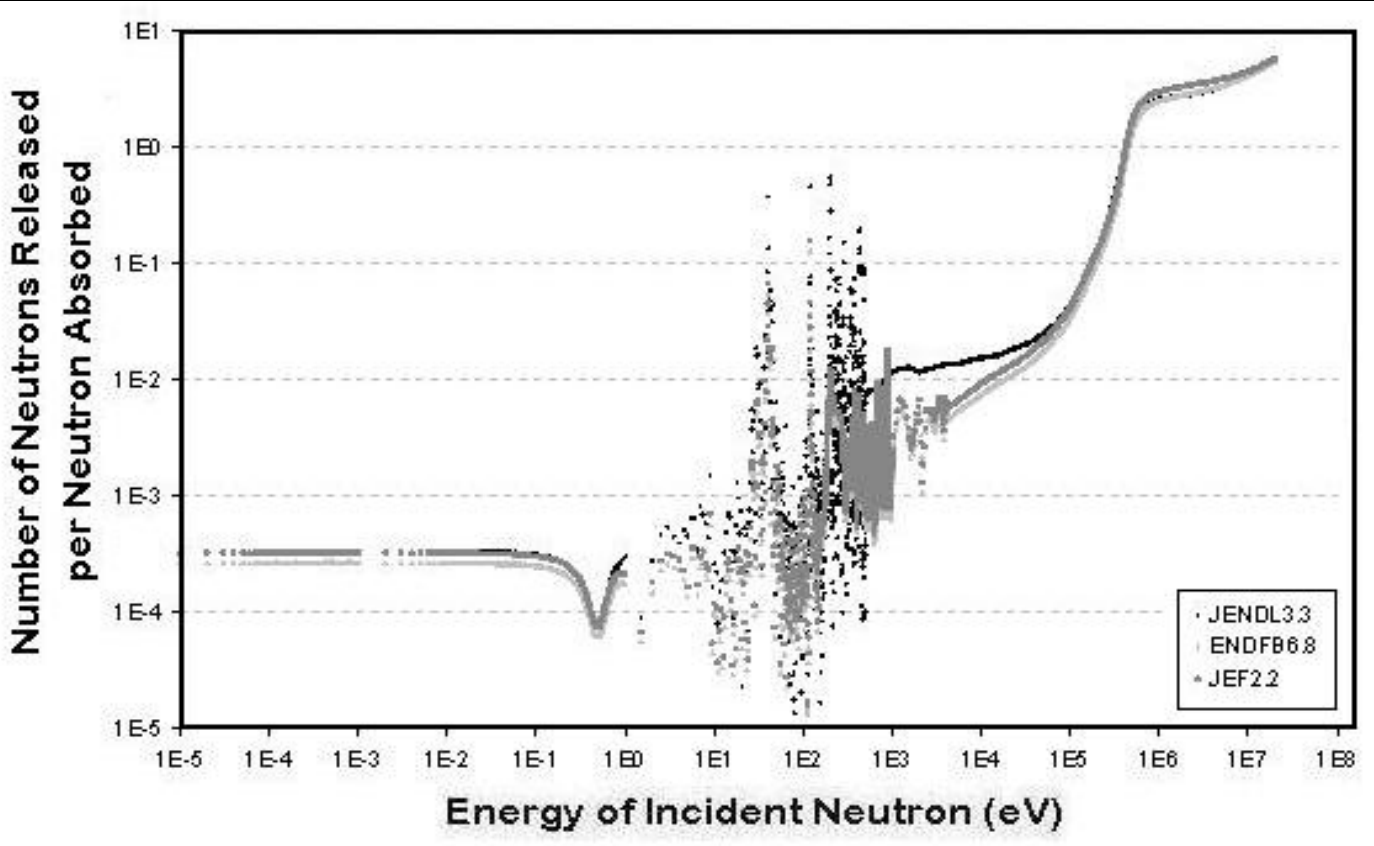

(a) ${ }^{237} \mathrm{~Np}$.

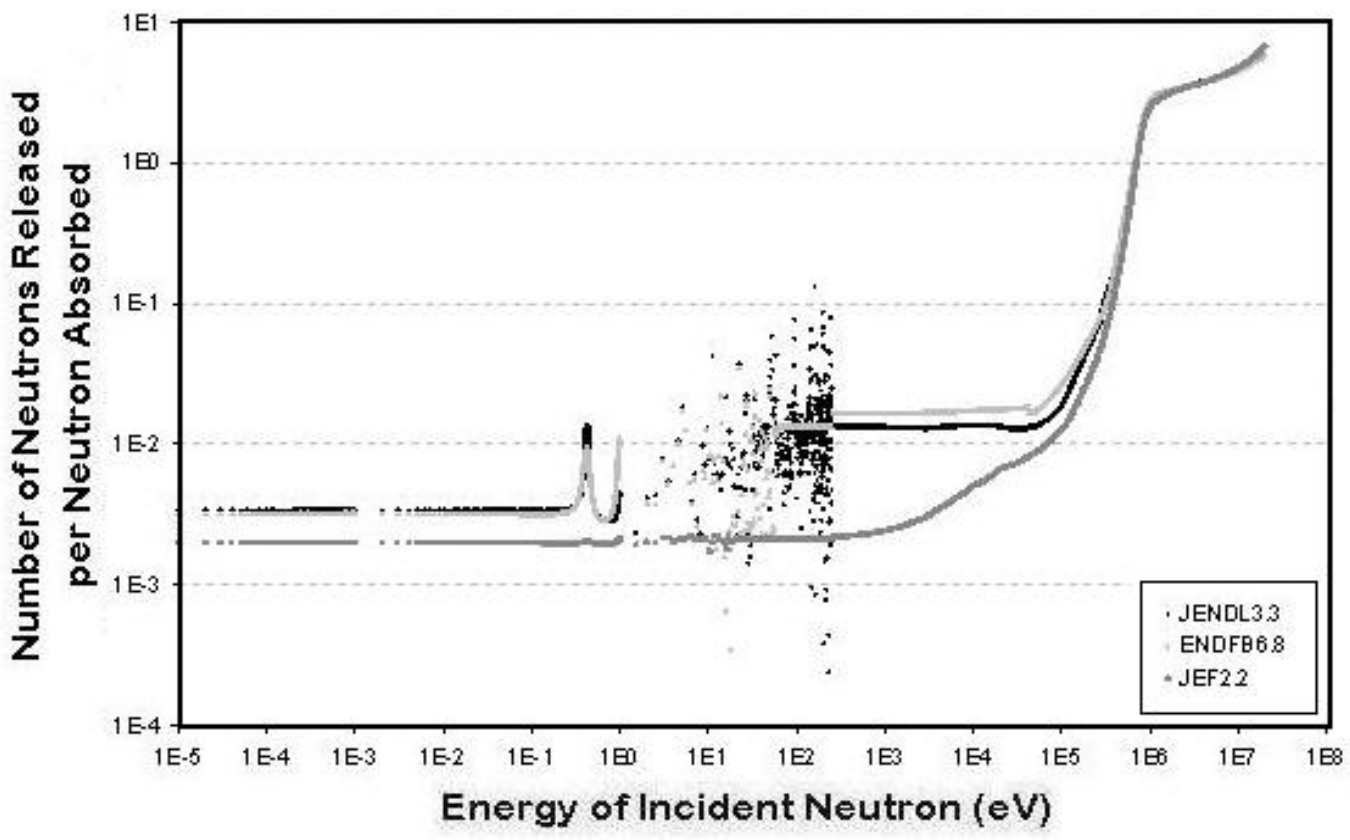

(b) ${ }^{243} \mathrm{Am}$.

Figure 8. Number of neutrons released per neutron absorbed in ${ }^{237} \mathrm{~Np}$ and ${ }^{243} \mathrm{Am}$. 
Utilization of MAs as a Fuel Component for Ultra-Long Life VHTR Configurations: Designs, Advantages and Limitations

Project 05-094

Final Scientific/Technical Report

The capture-to-fission rate ratio is shown in Figure 9. It can be seen that all MAs except ${ }^{242 \mathrm{~m}} \mathrm{Am},{ }^{243} \mathrm{Cm}$, and ${ }^{245} \mathrm{Cm}$ have larger values of $\alpha^{X}\left(E_{n}\right)$ than ${ }^{235} \mathrm{U}$ within the thermal energy range up to neutron energies of about $1.0 \mathrm{eV}$. Within the same energy range, ${ }^{238} \mathrm{U}$ has the largest values of $\alpha^{X}\left(E_{n}\right)$.

Table 9 shows the ranking of $\alpha^{X}\left(E_{n}\right)$ based on the order of magnitude. Using the magnitude ranking as an assessment metric, the existing discrepancies can be clearly shown. Similarly to individual microscopic cross sections, it can be seen that the overall sequence of the magnitudes of $\alpha^{X}\left(E_{n}\right)$ is consistent between the nuclear data files. Also as before, observed consistency between the evaluated data files doesn't mean that the data are accurate. Most probably, the same experimental data have been used as the basis for cross sections in different files.

Table 9. Ranking of the Capture-to-Fission Rate Ratio at Thermal Neutron Energies*

\begin{tabular}{|l|c|c|c|c|c|c|c|c|c|c|}
\hline File & ${ }^{235} \mathbf{U}$ & ${ }^{238} \mathbf{U}$ & ${ }^{237} \mathbf{N p}$ & ${ }^{241} \mathbf{A m}$ & ${ }^{242 m} \mathbf{A m}$ & ${ }^{243} \mathbf{A m}$ & ${ }^{242} \mathbf{C m}$ & ${ }^{243} \mathbf{C m}$ & ${ }^{244} \mathbf{C m}$ & ${ }^{245} \mathbf{C m}$ \\
\hline ENDF/B-6.8 & 9 & 1 & 2 & 4 & 8 & 3 & 6 & 7 & 5 & 10 \\
\hline JENDL-3.3 & 9 & 1 & 2 & 4 & 8 & 3 & 6 & 7 & 5 & 10 \\
\hline JEF-2.2 & 9 & 1 & 2 & 4 & 8 & 3 & 6 & 7 & 5 & 10 \\
\hline
\end{tabular}

*Ranking basis: 1 - largest value, 10 - smallest value.

Taking a closer look at the sequences of magnitudes of $\alpha^{X}\left(E_{n}\right)$ and $\eta^{X}\left(E_{n}\right)$, a reverse trend can also be noticed. Table 10 highlights this trend: The higher a nuclide ranks on the $\eta^{X}\left(E_{n}\right)$-scale, the lower it ranks on the $\alpha^{X}\left(E_{n}\right)$-scale. This is the general trend for all MAs with the exception of ${ }^{235} \mathrm{U}$ and ${ }^{243} \mathrm{Cm}$. However, as shown in Figure 10, in a consistent manner with the results discussed earlier, $\alpha^{X}\left(E_{n}\right)$ also varies amongst the evaluated nuclear data files.

Table 10. Ranking Comparison of the Elementary Reaction Rate Ratios*

\begin{tabular}{|l|c|c|c|c|c|c|c|c|c|c|c|}
\hline \multicolumn{2}{|l}{ File } & ${ }^{245} \mathbf{C m}$ & ${ }^{243} \mathbf{C m}$ & ${ }^{242 m} \mathbf{A m}$ & ${ }^{235} \mathbf{U}$ & ${ }^{242} \mathbf{C m}$ & ${ }^{244} \mathbf{C m}$ & ${ }^{241} \mathbf{A m}$ & ${ }^{243} \mathbf{A m}$ & ${ }^{237} \mathbf{N p}$ & ${ }^{238} \mathbf{U}$ \\
\hline \multirow{2}{*}{ ENDF/B-6.8 } & $\eta$ & 1 & 2 & 3 & 4 & 5 & 6 & 7 & 8 & 9 & 10 \\
\cline { 2 - 14 } & $\alpha$ & 10 & 7 & 8 & 9 & 6 & 5 & 4 & 3 & 2 & 1 \\
\hline \multirow{2}{*}{ JENDL-3.3 } & $\eta$ & 1 & 2 & 3 & 4 & 5 & 6 & 7 & 8 & 9 & 10 \\
\cline { 2 - 14 } & $\alpha$ & 10 & 7 & 8 & 9 & 6 & 5 & 4 & 3 & 2 & 1 \\
& $\eta$ & - & - & - & 1 & - & - & - & 2 & 3 & 4 \\
\cline { 2 - 13 } & $\alpha$ & 10 & 7 & 8 & 9 & 6 & 5 & 4 & 3 & 2 & 1 \\
\hline
\end{tabular}

*Ranking basis: 1 - largest value, 10 - smallest value. 


\section{STn \\ Research Initiative}

Utilization of MAs as a Fuel Component for Ultra-Long Life

VHTR Configurations: Designs, Advantages and Limitations

Project 05-094

Final Scientific/Technical Report

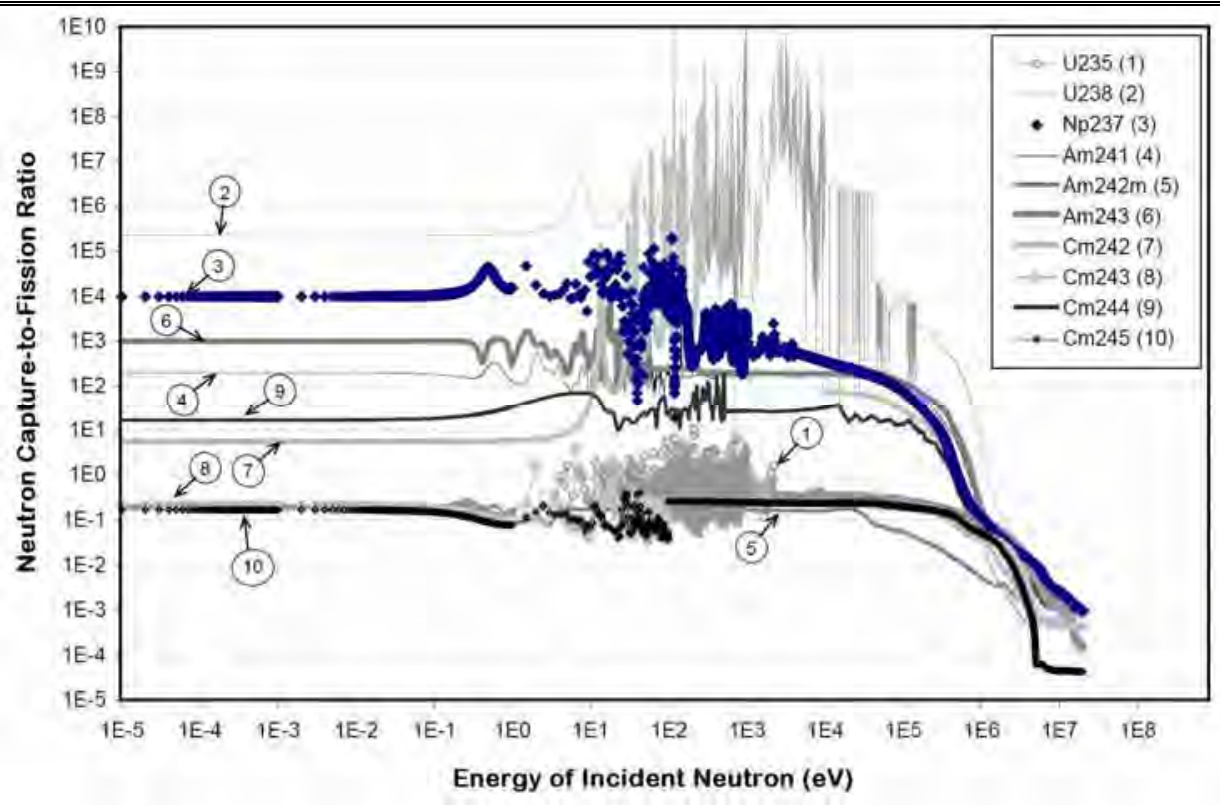

(a) ENDF/B-6.8.

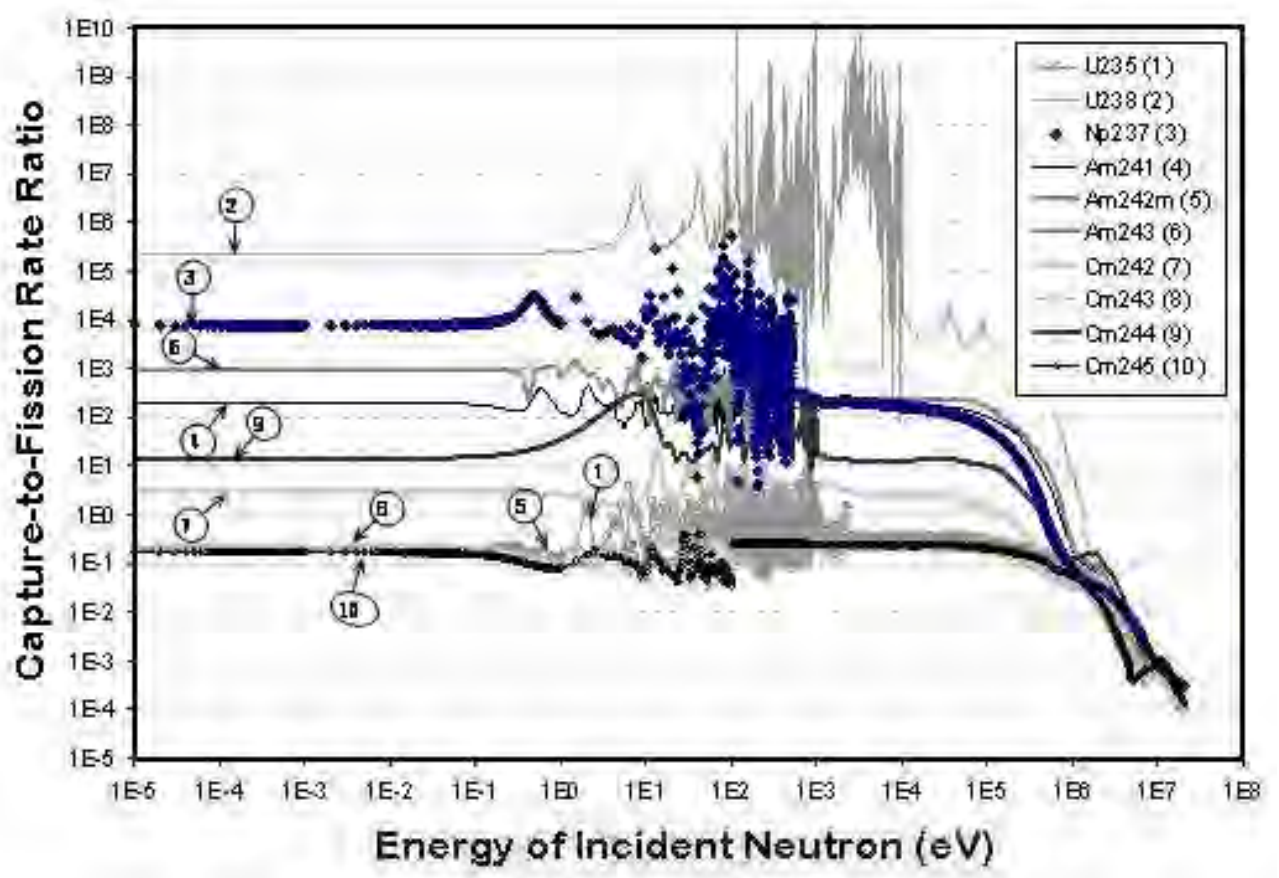

(b) JENDL-3.3. 
Utilization of MAs as a Fuel Component for Ultra-Long Life VHTR Configurations: Designs, Advantages and Limitations

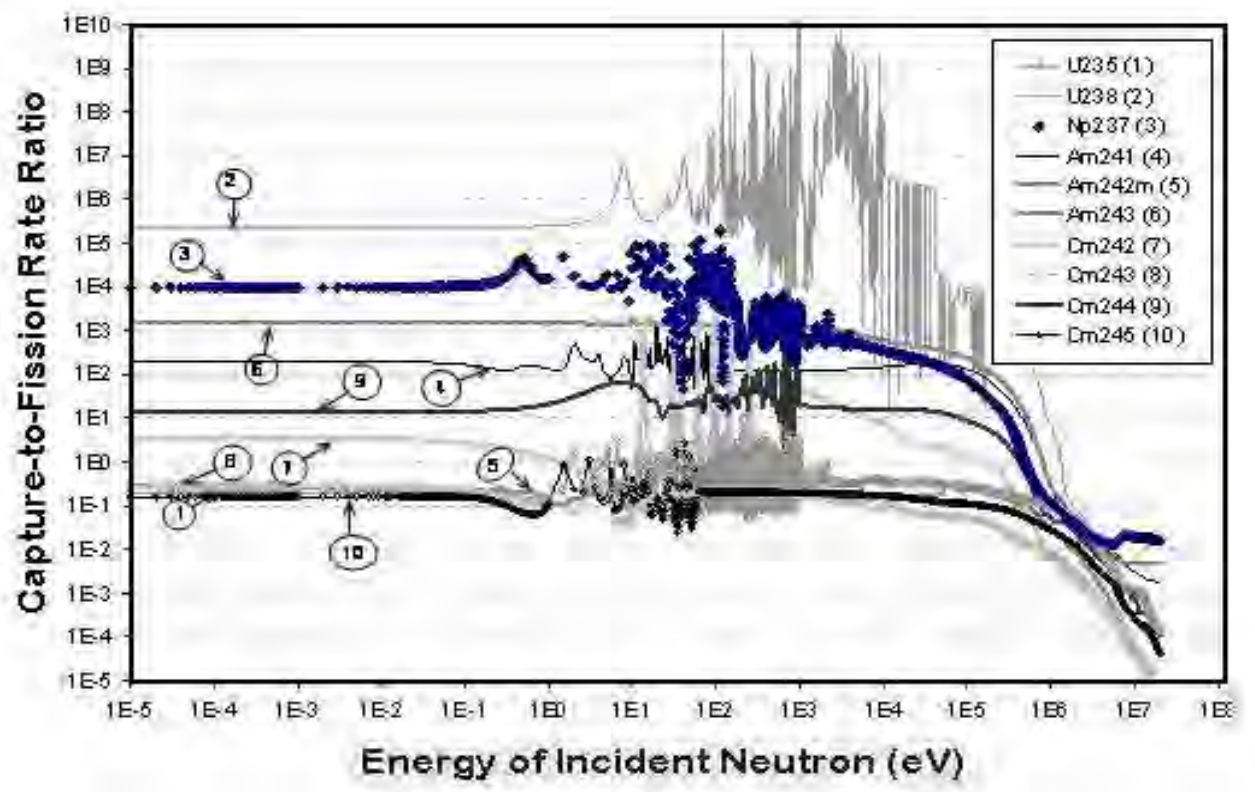

(c) JEF-2.2.

Figure 9. Capture-to-fission rate ratio (ENDF/B-6.8, JENDL-3.3, JEF-2.2).

The delayed neutron fractions of MAs are shown in Figure 11. It has to be emphasized that $\beta^{X}\left(E_{n}\right)$ of MAs are substantially smaller than the corresponding reference values for ${ }^{235} \mathrm{U}$ and ${ }^{238} \mathrm{U}$. Table 11 summarizes the representative delayed neutron fractions of MAs and relates their values to the corresponding values for ${ }^{235} \mathrm{U}$ and ${ }^{238} \mathrm{U}$.

Table 11. Delayed Neutron Fractions of MAs at Thermal Neutron Energies

\begin{tabular}{|c|c|c|c|c|c|c|}
\hline \multirow[t]{2}{*}{ Nuclide } & \multicolumn{3}{|c|}{ ENDF/B-6.8 } & \multicolumn{3}{|c|}{ JENDL-3.3 } \\
\hline & $\beta, 10^{4}$ & $\%$ of ${ }^{235} U$ & $\%$ of ${ }^{238} U$ & $\beta, 10^{4}$ & $\%$ of ${ }^{235} U$ & $\%$ of ${ }^{238} U$ \\
\hline${ }^{235} \mathbf{U}$ & 68.54 & 100.00 & 38.82 & 65.06 & 100.00 & 34.92 \\
\hline${ }^{238} \mathbf{U}$ & 176.56 & 257.60 & 100.00 & 186.29 & 286.34 & 100.00 \\
\hline${ }^{237} \mathbf{N p}$ & 41.01 & 59.83 & 23.23 & 46.12 & 70.89 & 24.76 \\
\hline${ }^{241} \mathrm{Am}$ & 13.18 & 19.23 & 7.46 & 16.01 & 24.61 & 8.59 \\
\hline${ }^{242 \mathrm{~m}} \mathrm{Am}$ & 21.14 & 30.84 & 11.97 & 19.87 & 30.54 & 10.67 \\
\hline${ }^{243} \mathrm{Am}$ & 24.29 & 35.44 & 13.76 & 26.49 & 40.72 & 14.22 \\
\hline${ }^{242} \mathrm{Cm}$ & 3.96 & 5.78 & 2.24 & 6.43 & 9.88 & 3.45 \\
\hline${ }^{243} \mathrm{Cm}$ & 8.77 & 12.80 & 4.97 & 8.77 & 13.48 & 4.71 \\
\hline${ }^{244} \mathrm{Cm}$ & NA & - & - & 13.41 & 20.61 & 7.20 \\
\hline${ }^{245} \mathrm{Cm}$ & 17.80 & 25.97 & 10.08 & 17.80 & 27.36 & 9.55 \\
\hline
\end{tabular}




\section{Utilization of MAs as a Fuel Component for Ultra-Long Life}

VHTR Configurations: Designs, Advantages and Limitations

Final Scientific/Technical Report

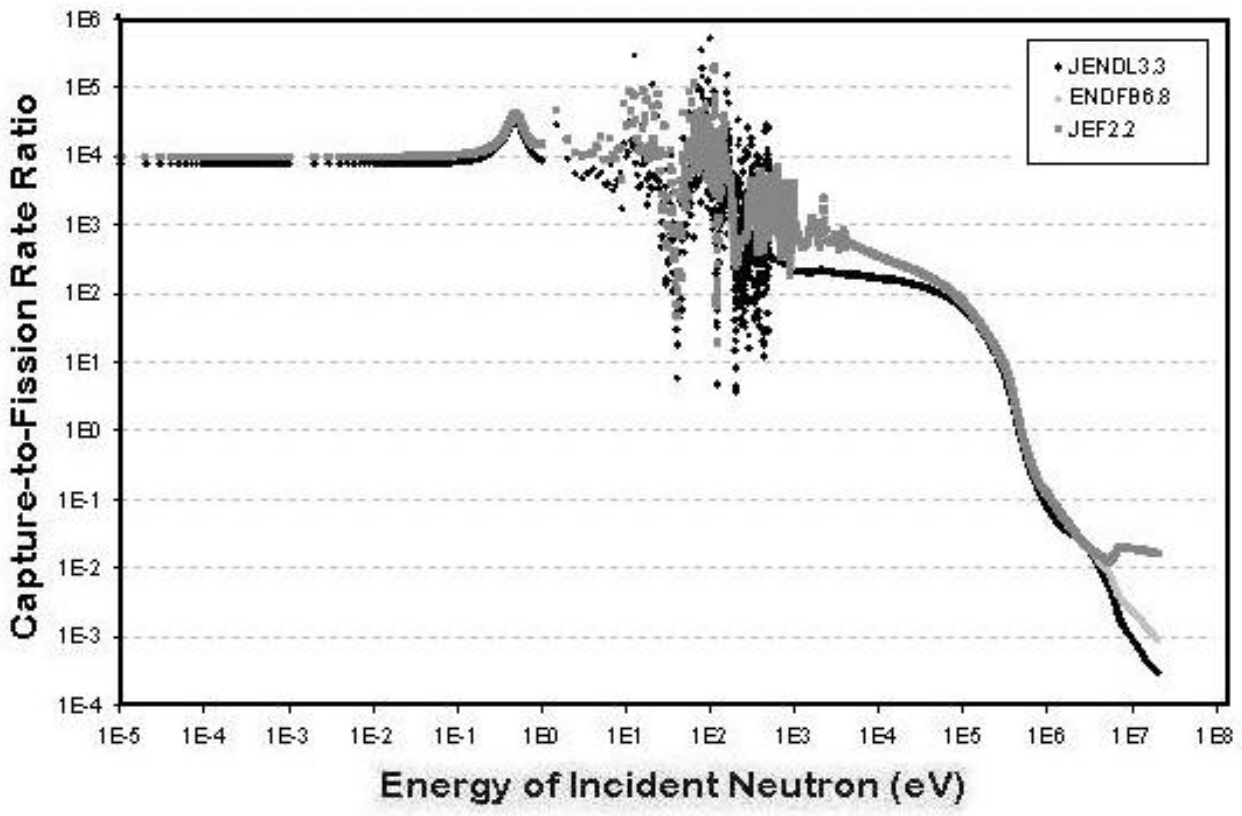

(a) ${ }^{237} \mathrm{~Np}$.

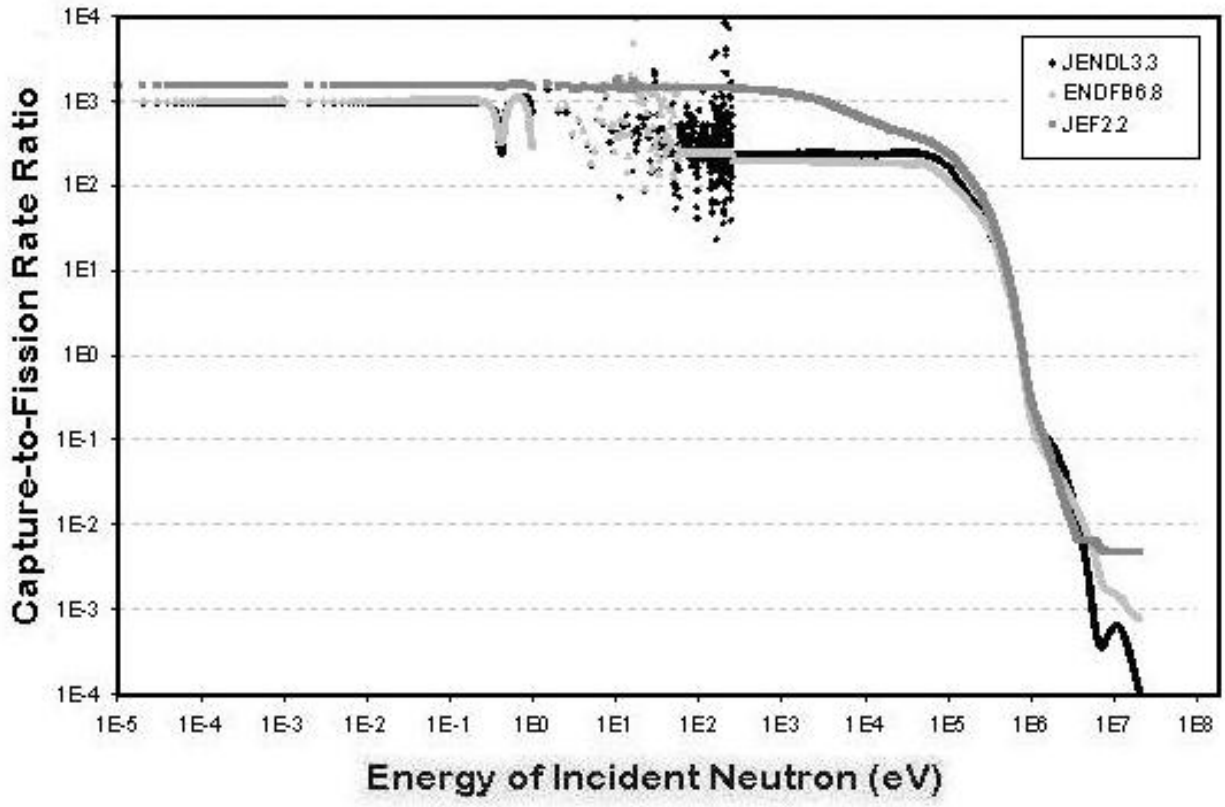

(b) ${ }^{243} \mathrm{Am}$. 
Utilization of MAs as a Fuel Component for Ultra-Long Life

VHTR Configurations: Designs, Advantages and Limitations

Final Scientific/Technical Report

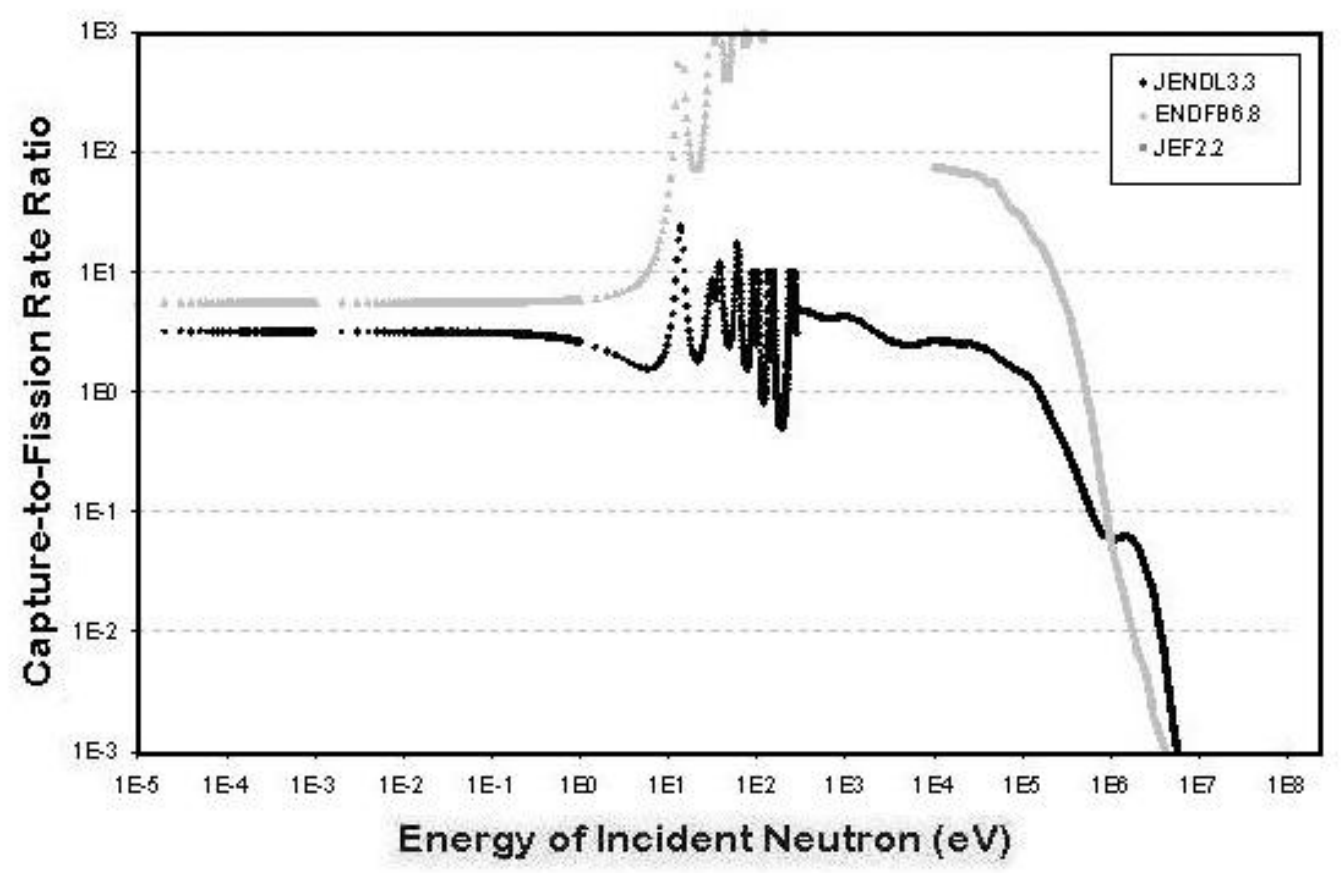

(c) ${ }^{242} \mathrm{Cm}$.

Figure 10. Capture-to-fission rate ratios of ${ }^{237} \mathrm{~Np},{ }^{243} \mathrm{Am}$ and ${ }^{242} \mathrm{Cm}$.

Figure 12 shows the delayed neutron fractions of ${ }^{237} \mathrm{~Np},{ }^{243} \mathrm{Am}$, and ${ }^{242} \mathrm{Cm}$. The values of $\beta^{X}\left(E_{n}\right)$ vary amongst the evaluated nuclear data files.

As expected, the effects of the discrepancies between the nuclear data in ENDF/B-6.8, JENDL-3.3 and JEF-2.2 have propagated to the calculated basic neutronics characteristics. The elementary reaction rate ratios, $\alpha^{X}\left(E_{n}\right), \beta^{X}\left(E_{n}\right)$, and $\eta^{X}\left(E_{n}\right)$, are relatively consistent in their rankings. However, absolute values of the characteristics vary significantly amongst the evaluated nuclear data files. These effects contribute to the existing uncertainties and limit reliability of the analysis by causing differences in reactor physics characteristics. 


\section{STR \\ Research Initiative}

Utilization of MAs as a Fuel Component for Ultra-Long Life

VHTR Configurations: Designs, Advantages and Limitations

Project 05-094

Final Scientific/Technical Report

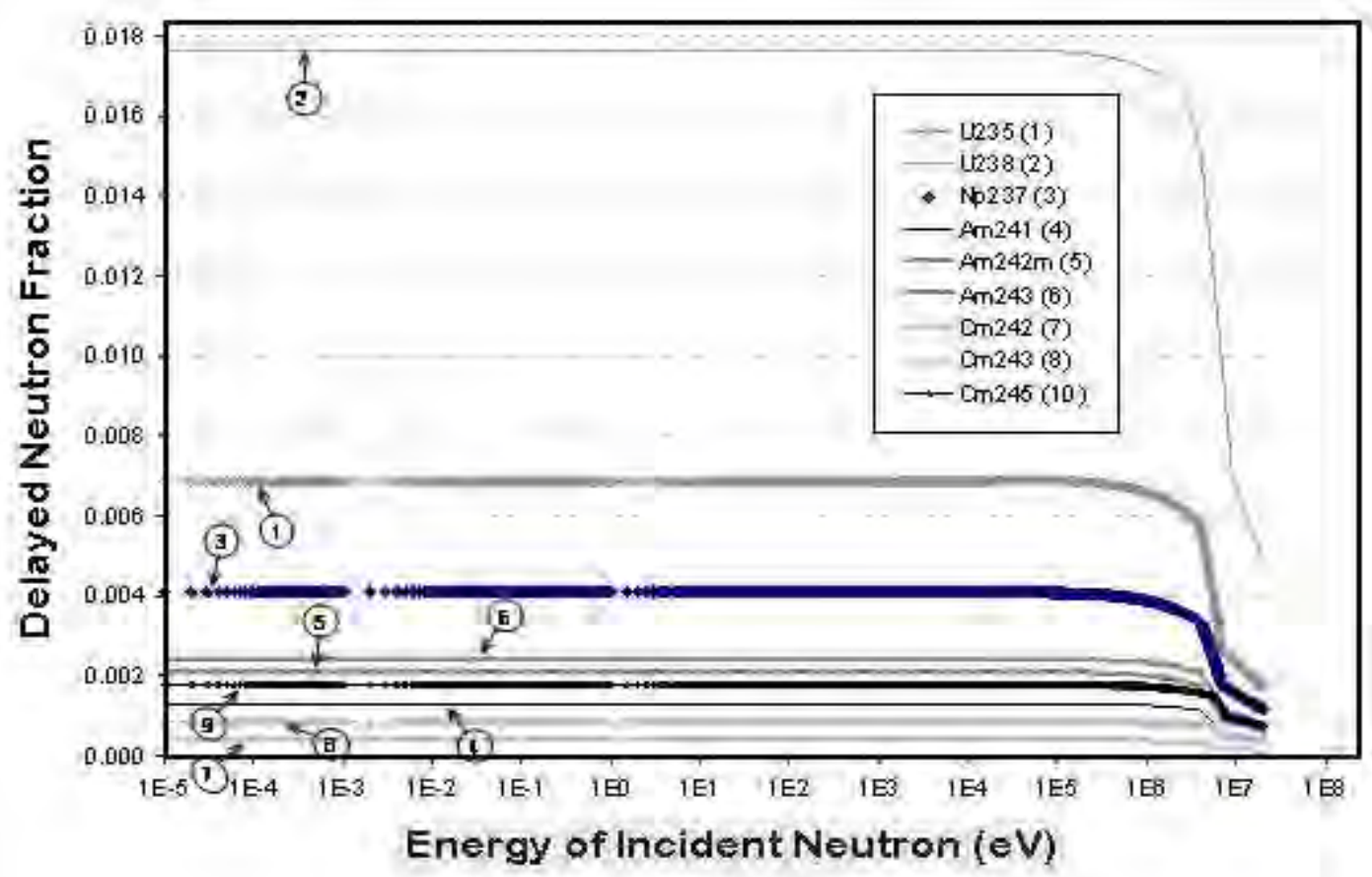

(a) ENDF/B-6.8.

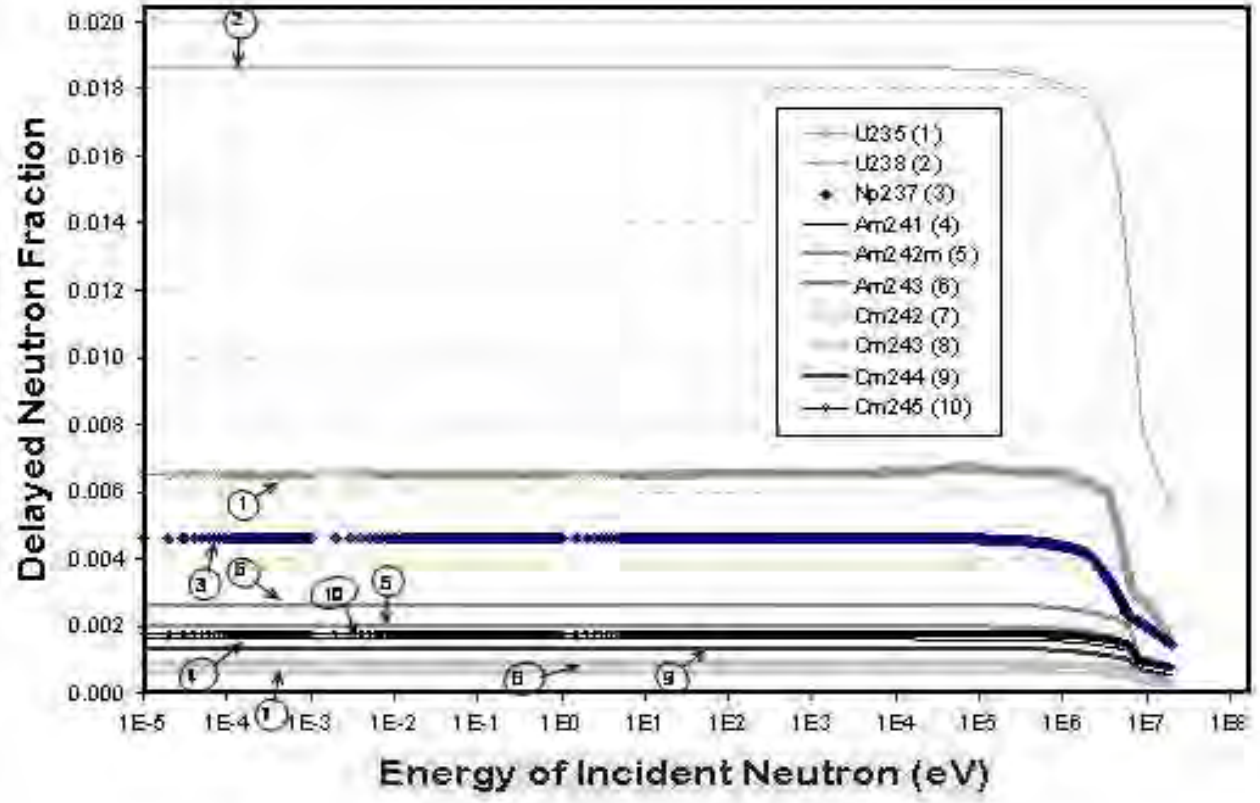

(b) JENDL-3.3.

Figure 11. Delayed neutron fractions of MAs (ENDF/B-6.8 and JENDL-3.3). 
Utilization of MAs as a Fuel Component for Ultra-Long Life

VHTR Configurations: Designs, Advantages and Limitations

Project 05-094

Final Scientific/Technical Report

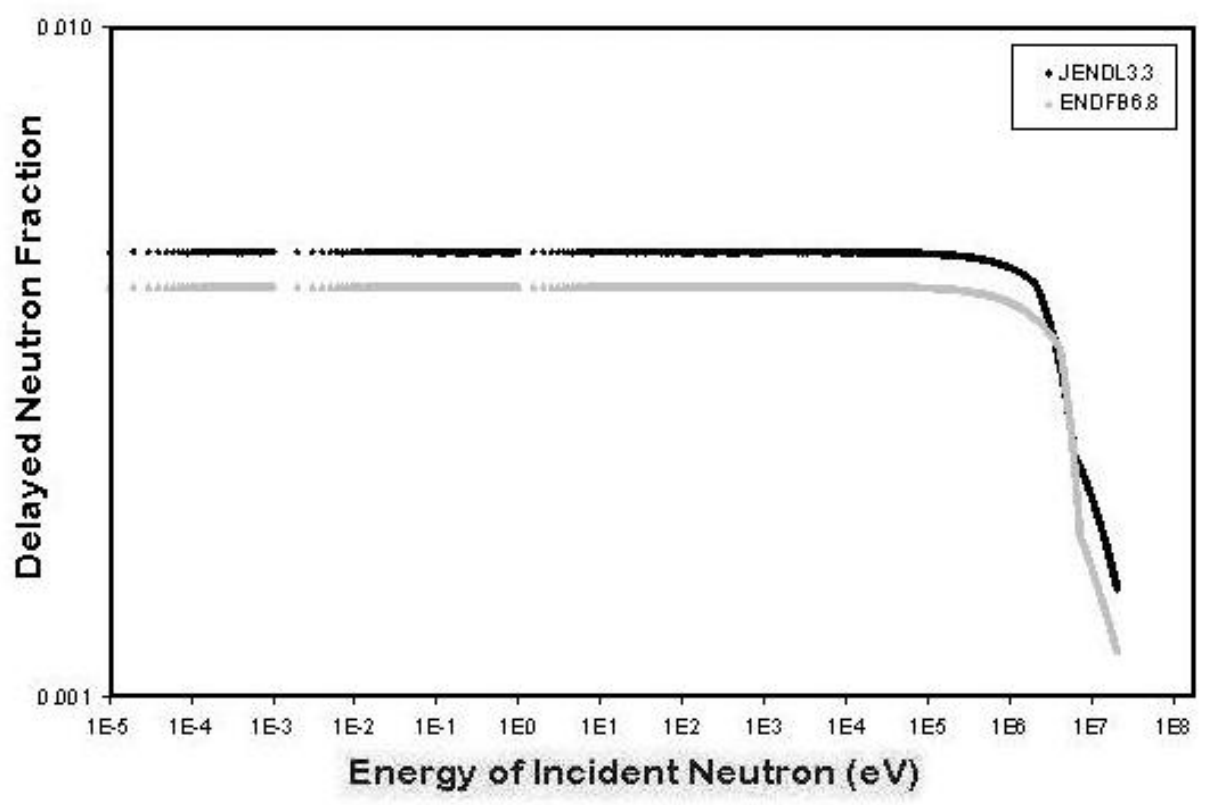

(a) ${ }^{237} \mathrm{~Np}$.

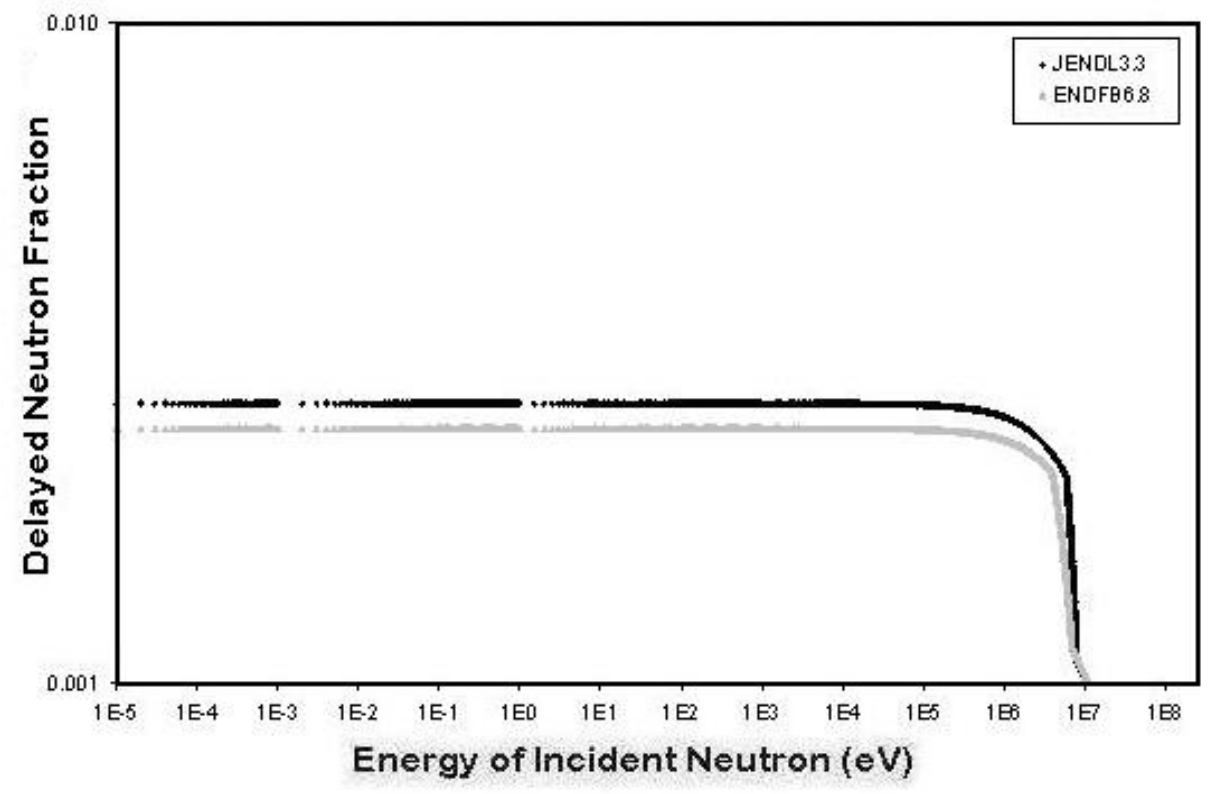

(b) ${ }^{243} \mathrm{Am}$. 
Utilization of MAs as a Fuel Component for Ultra-Long Life

VHTR Configurations: Designs, Advantages and Limitations

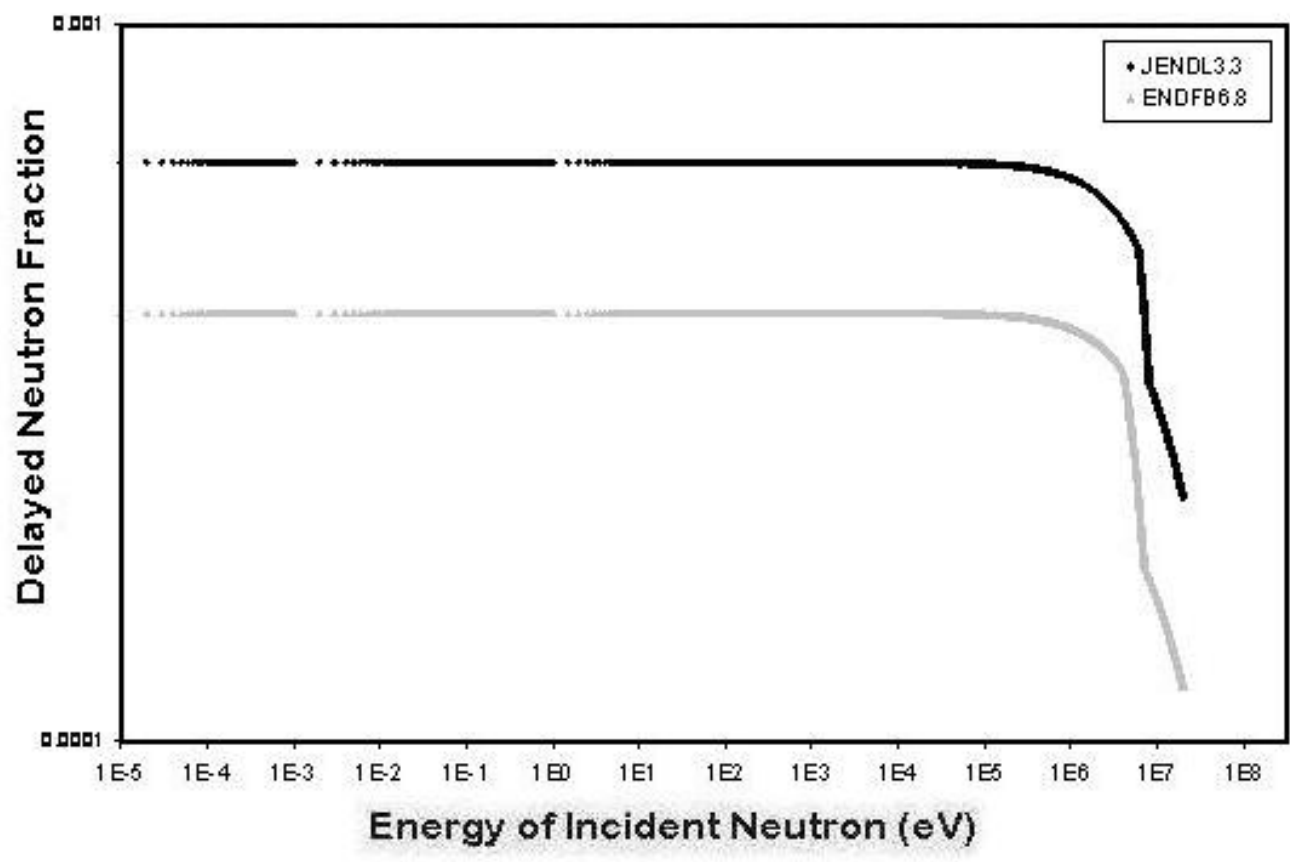

(c) ${ }^{242} \mathrm{Cm}$.

Figure 12. Delayed neutron fraction of ${ }^{237} \mathrm{~Np},{ }^{243} \mathrm{Am}$ and ${ }^{242} \mathrm{Cm}$.

\subsection{Sensitivity of the VHTR Core Performance to the Neutron-Kernel Interactions}

As defined above, the Dancoff correction factor is an important parameter that characterizes a probability for neutrons to interact with fuel kernels. To provide an understanding of how this parameter affects the core performance a sensitivity analysis with respect to the Dancoff correction factor was conducted. This entails performing multiple criticality calculations for a range of Dancoff correction factors followed by the calculations of dimensionless sensitivity indicators, $S_{m, n}=S_{k, \text { Dancoff }}$, for variations of $k_{\text {eff }}$ due to variations of Dancoff correction factor.

The sensitivity analysis was performed for the HTR-10 core and the critical HTTR core, which was experimentally determined to have control rod insertion depths of $177.5 \mathrm{~cm}$. The HTTR computational model was altered to match this case.

A total of nine Dancoff factors ranging from 0.20 (typical pebble-bed core range) to 0.95 (upper range for prismatic cores) were evaluated at two temperatures, $300 \mathrm{~K}$ and $700 \mathrm{~K}$. The results are summarized in Table 12. The Dancoff factor for the HTTR core is expected to be in 


\section{Utilization of MAs as a Fuel Component for Ultra-Long Life} VHTR Configurations: Designs, Advantages and Limitations

the 0.7 to 0.9 range. As also shown in Table 12, detailed comparisons for the HTR-10 pebble-bed core and other VHTRs can be performed in a similar manner.

Table 12. Dancoff Factors and Corresponding Effective Multiplication

\begin{tabular}{|c|c|c|c|c|c|c|c|c|c|}
\hline \multirow[t]{3}{*}{$\begin{array}{l}\text { Dancoff } \\
\text { Factor }\end{array}$} & \multicolumn{6}{|c|}{$\begin{array}{c}\text { HTTR } \\
\text { (Control Rods at Critical Position Determined at 300K) }\end{array}$} & \multirow{2}{*}{\multicolumn{3}{|c|}{$\begin{array}{c}\text { HTR-10 } \\
\text { (Control Rods Withdrawn, } \\
\text { Homogeneous Core) } \\
\text { 300K }\end{array}$}} \\
\hline & \multicolumn{3}{|c|}{$300 \mathrm{~K}$} & \multicolumn{3}{|c|}{$700 \mathrm{~K}$} & & & \\
\hline & $k_{\text {eff }}$ & $\delta_{k}$ & $\begin{array}{c}S_{k, \text { Dancoff }} \\
(\mathbf{\%})\end{array}$ & $k_{e f f}$ & $\delta_{k}$ & $\begin{array}{c}S_{k, \text { Dancoff }} \\
\text { (\%) }\end{array}$ & $k_{e f f}$ & $\delta_{k}$ & $\begin{array}{c}S_{k, \text { Dancoff }} \\
\mathbf{( \% )}\end{array}$ \\
\hline 0.20 & 0.9507 & 0.0052 & +1.1 & 0.8858 & 0.0027 & +0.6 & 1.1492 & 0.0011 & +0.2 \\
\hline 0.30 & 0.9559 & 0.0097 & +3.0 & 0.8885 & 0.0075 & +2.5 & 1.1503 & 0.0016 & +0.4 \\
\hline 0.40 & 0.9656 & 0.0054 & +2.2 & 0.8960 & 0.0011 & +0.5 & 1.1519 & 0.0022 & +0.8 \\
\hline 0.50 & 0.971 & 0.0021 & +1.1 & 0.8971 & 0.0125 & +7.0 & 1.1541 & 0.0027 & +1.2 \\
\hline 0.60 & 0.9731 & 0.008 & +4.9 & 0.9096 & 0.0024 & +1.6 & 1.1568 & 0.0033 & +1.7 \\
\hline 0.70 & 0.9811 & 0.014 & +10.0 & 0.9120 & 0.0175 & +13.4 & 1.1601 & 0.0039 & +2.3 \\
\hline 0.80 & 0.9951 & 0.0152 & +12.2 & 0.9295 & 0.0142 & +12.2 & 1.1640 & 0.0044 & +3.0 \\
\hline 0.90 & 1.0103 & 0.0117 & +20.8 & 0.9437 & 0.0069 & +13.2 & 1.1684 & 0.0024 & +3.7 \\
\hline
\end{tabular}




\section{CONFIGURATION ADJUSTMENT POTENTIAL OF VHTRs WITH ACTINIDE FUELS}

In the conventional closed fuel cycle, recovered minor actinides are treated and stored as high level wastes. Partitioning and transmutation engineering technologies offer their incineration and may potentially provide additional fuel supply. The proposed earlier Deep-Burn concept suggests thermal neutron transmutation of minor actinides in the HTGR prismatic cores taking advantage of actinide nuclear properties.

The VHTR (Very High Temperature Reactor) system is the nearest Generation IV concept. The objective of the awarded U.S. DOE University NERI Project is to assess the possibility, advantages and limitations of VHTRs with minor actinides as a fuel component. Since VHTR core designs permit flexibility in component configuration, fuel utilization and management, it is possible to improve fissile properties of minor actinides by neutron spectrum shifting through configuration adjustments. As a result, under certain spectral conditions minor actinides would be able to contribute to the core neutron balance compensating for fuel depletion effects through their chain transformations. The resulting self-stabilization of advanced actinide fuels should allow prolonged operation on a single fuel loading up to ultra-long lifetimes that are comparable with the entire reactor lifetime.

In this project, the VHTR systems are being developed and analyzed focusing on control, dynamics, safety, and proliferation-resistance. The main advantages of the resulting configurations are their inherent capabilities for utilization of minor actinides from spent LWR fuel and reduction of spent fuel flows and handling per unit of produced energy. Consequently, if widely deployed, the developed designs would allow reducing the long-term radiotoxicity and heat load of high-level wastes sent to a geologic repository and enable recovery of the energy contained in spent fuel. 


\title{
Utilization of MAs as a Fuel Component for Ultra-Long Life
} VHTR Configurations: Designs, Advantages and Limitations

\author{
Configuration Adjustment Potential of the VHTR Prismatic Cores with Advanced Actinide Fuels \\ Pavel V. Tsvetkov, David E. Ames II, and Ayodeji B. Alajo \\ Department of Nuclear Engineering, Texas A\&M University, 129 Zachry Engineering Center, MS 3133 TAMU \\ College Station, TX, 77843-3133,E-mail: tsvetkov@.tamu.edu
}

\section{INTRODUCTION}

In the conventional closed fuel cycle, recovered minor actinides are treated and stored as high level wastes. Partitioning and transmutation engineering technologies offer their incineration and may potentially provide additional fuel supply. [1] The proposed earlier Deep-Burn concept suggests thermal neutron transmutation of minor actinides in the HTGR prismatic cores taking advantage of actinide nuclear properties. [2]

The VHTR (Very High Temperature Reactor) system is the nearest Generation IV concept. The objective of the awarded U.S. DOE University NERI Project is to assess the possibility, advantages and limitations of VHTRs with minor actinides as a fuel component. Since prismatic core designs permit flexibility in component configuration, fuel utilization and management, it is possible to improve fissile properties of minor actinides by neutron spectrum shifting through configuration adjustments. As a result, under certain spectral conditions minor actinides would be able to contribute to the core neutron balance compensating for fuel depletion effects through their chain transformations. The resulting self-stabilization of advanced actinide fuels should allow prolonged operation on a single fuel loading up to ultra-long lifetimes that are comparable with the entire reactor lifetime.

In this project, the VHTR systems are being developed and analyzed focusing on control, dynamics, safety, and proliferation-resistance. The main advantages of the resulting configurations are their inherent capabilities for utilization of minor actinides from spent LWR fuel and reduction of spent fuel flows and handling per unit of produced energy. Consequently, if widely deployed, the developed designs would allow reducing the long-term radiotoxicity and heat load of high-level wastes sent to a geologic repository and enable recovery of the energy contained in spent fuel.

\section{PHYSICS OF THE VHTR PRISMATIC CORE}

Specific features of the VHTR prismatic core are coated micro-particles containing fuel in their kernels, helium as a coolant, and graphite as moderator and structural material. This combination of materials results in the unique physics and in the excellent performance characteristics.

Although it is a less pronounced effect than in pebble-bed cores, in prismatic cores presence of coated fuel particles, compacts, prismatic graphite blocks with coolant passages, internal support structures and control rods results in multi-heterogeneity phenomena. It is possible to improve fissile properties of minor actinides by neutron spectrum shifting through configuration adjustments. Consequently, small reactivity swings will be sustained in the VHTR configurations with minor actinides over prolonged irradiation periods up to the entire reactor lifetime.

Coated fuel particles reliably retain fission products up to $1000^{\circ} \mathrm{C}$ and higher. Because the reactor core is composed entirely of high-temperature materials, very high outlet gas temperatures are potentially achievable. Furthermore, due to refractory nature of materials, these configurations are relatively resistant to power trips and loss-of-coolant accidents. The present study is focused on the VHTR configurations due to their versatility with respect to fuel cycles. It is possible to choose between a wide range of fuel enrichments and forms including advanced actinide fuels.

\section{VALIDATION \& VERIFICATION OF THE MODEL}

The computational modeling is performed using state-of-the-art computer codes that allow realistic modeling of the VHTR configurations. [3,4] Figure 1 shows the developed $3 \mathrm{D}$ whole-core model as it was applied to the HTTR benchmark configurations.

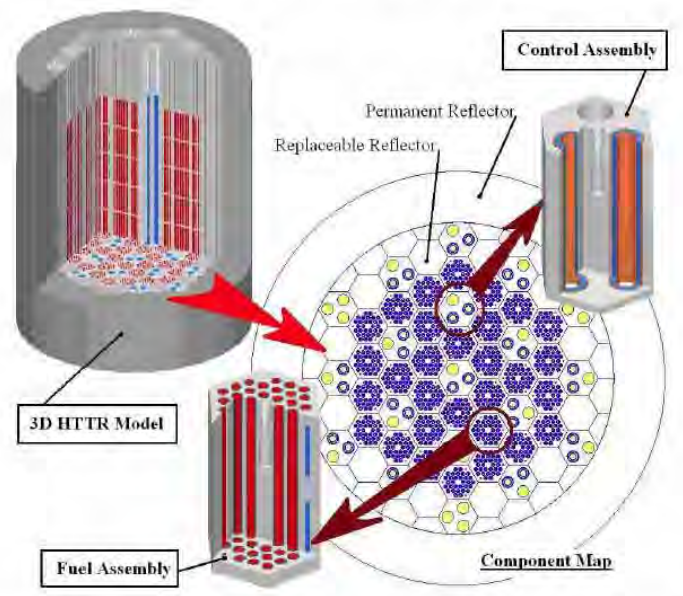

Fig. 1. 3D whole-core VHTR model - HTTR benchmark (KENO3D visual).

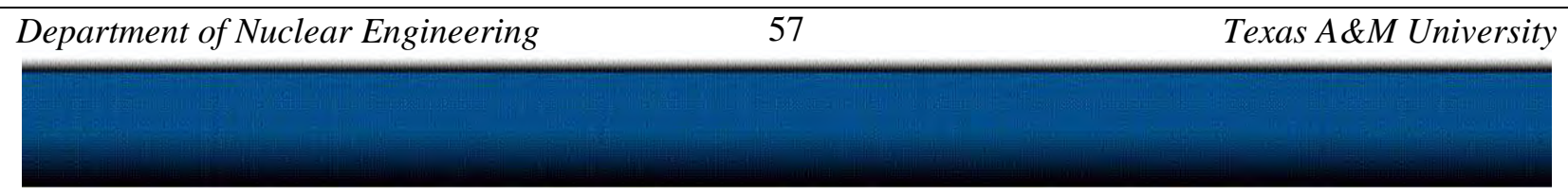




\section{Utilization of MAs as a Fuel Component for Ultra-Long Life} VHTR Configurations: Designs, Advantages and Limitations

As the basis for development of the benchmark test problems, the project uses the actual test results of the HTTR program. [5] The HTTR benchmark program targets $10 \%$ prediction accuracy or better in computational studies. Thus, as illustrated in Table I, the obtained results are in agreement with the available experimental data and meet the HTTR program targets. The larger discrepancy between experimental evaluations and computational results for the HTTR core with fully inserted control rods is due to ambiguity of available information regarding control rods and the corresponding core configurations.

TABLE I. Benchmark Evaluation of the VHTR Modeling

\begin{tabular}{|l|l|l|c|}
\hline \multirow{2}{*}{$\begin{array}{l}\text { HTTR } \\
\text { Control }\end{array}$} & \multicolumn{3}{|c|}{ Core Multiplication } \\
\cline { 2 - 4 } & Experiment & Model & $\varepsilon(\%)$ \\
\hline Out & $1.1363 \pm 0.041$ & $1.1255 \pm 0.0018$ & -0.95 \\
\hline In & $0.6850 \pm 0.010$ & $0.7254 \pm 0.0018$ & +5.90 \\
\hline
\end{tabular}

\section{VHTR PERFORMANCE ANALYSIS}

The VHTR multi-heterogeneity features allow material separation by using different types of microparticles and graphite blocks. The degree of heterogeneity and the moderator-to-fuel ratio can be adjusted to achieve desirable spectrum shifting. As illustrated in Fig. 2, depending on dominant neutron energies in the considered core configurations, neptunium, americium and curium may serve not only as burnable poisons (high absorption (capture) but low neutron yield) but also as high-yield fuel materials contributing to self-stabilization effects that allow prolonged single-loading lifetimes.

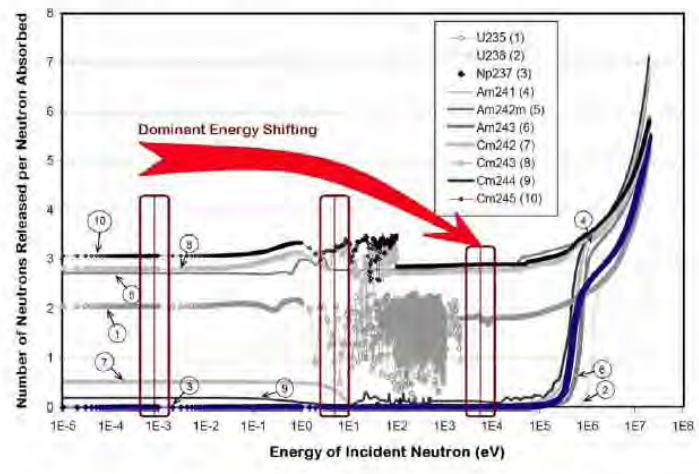

Fig. 2. Actinide properties and spectrum shifting.

\section{CONCLUSIONS (CURRENT STATUS)}

The project is currently within its first year of studies. Whole-core/system 3D models with multi-heterogeneity treatments and properties databases of actinide compounds are being developed. Studies of the actinide- fueled VHTR configurations are underway. As presented in this paper, the analysis indicates promising performance. Based on the up-to-date progress, accomplishment of the project objectives is anticipated within the project time frame.

\section{ACKNOWLEDGMENTS}

This paper is based upon work supported by the U.S. Department of Energy under Award Number DE-FC0705ID14655 (05-094).

\section{DISCLAIMER}

This paper was prepared as an account of work sponsored by an agency of the United States Government. Neither the United States Government nor any agency thereof, nor any of their employees, makes any warranty, express or implied, or assumes any legal liability or responsibility for the accuracy, completeness, or usefulness of any information disclosed. The views and opinions of authors expressed herein do not necessarily state or reflect those of the United States Government or any agency thereof.

\section{REFERENCES}

1. "Report to Congress on Advanced Fuel Cycle Initiative: The Future Path for Advanced Spent Fuel Treatment and Transmutation Research", 03GA50439-06, January, 2003, Office of Nuclear Energy, Science, and Technology, U.S. DOE (2003).

2. C. RODRIGUEZ, A. BAXTER, D. MCEACHERN, M. FIKANI, F. VENNERI, "Deep-Burn: Making Nuclear Waste Transmutation Practical", Nucl. Eng. Design, 2805, 1 (2003).

3. "SCALE: A Modular Code System for Performing Standardized Computer Analyses for Licensing Evaluation", ORNL/TM-2005/39, Ver. 5, April 2005, Oak Ridge National Laboratory, Oak Ridge, Tennessee (2005).

4. J. VALKO, P. V. TSVETKOV, J. E. HOOGENBOOM, "Calculation of the Dancoff Factor for Pebble-Bed Reactors", Nucl. Sci. Eng., 135, 304 (2000).

5. "Evaluation of High Temperature Gas-cooled Reactor Performance: Benchmark Analysis Related to Initial Testing of the HTTR and HTR-10", IAEATECDOC-1382, International Atomic Energy Agency, Vienna, Austria (2003).

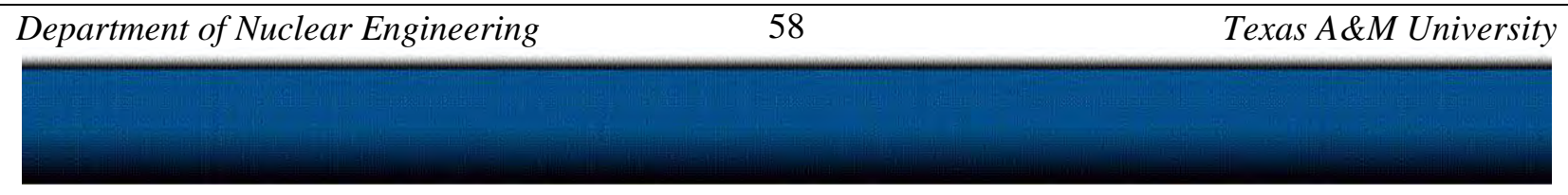




\title{
Utilization of MAs as a Fuel Component for Ultra-Long Life
} VHTR Configurations: Designs, Advantages and Limitations

\author{
Analysis of the Pebble-Bed VHTR Spectrum Shifting Capabilities for Advanced Fuel Cycles \\ Pavel V. Tsvetkov, Megan L. Pritchard, David E. Ames II, and Ayodeji B. Alajo \\ Department of Nuclear Engineering, Texas A\&M University, 129 Zachry Engineering Center, MS 3133 TAMU \\ College Station,TX, 77843-3133,E-mail:tsvetkov@.tamu.edu
}

\section{INTRODUCTION}

Current waste management strategies presume actinide recycle in the conventional closed fuel cycle by reprocessing of spent LWR fuel. Partitioning and transmutation of minor actinides is a very challenging option but it could have a positive impact on the global nuclear fuel cycle due to resulting elimination of hazardous nuclear wastes. [1] In addition, partitioning and transmutation engineering technologies may potentially serve as a source of constituents for advanced actinide fuels assuring sustainable nuclear energy development.

The U.S. DOE University NERI Project focuses on performance characteristics of VHTRs (Very High Temperature Reactors) with fuel loadings containing minor actinides. Both pebble-bed and prismatic core designs are taken into account. This paper presents the analysis of pebble-bed configurations whereas prismatic core options are discussed elsewhere. [2]

\section{VHTR PEBBLE-BED CONFIGURATIONS}

The pebble-bed VHTR has features contributing to fundamental differences in reactor physics of this system in comparison with other reactor concepts. Due to the use of coated micro-particle fuel, at least two levels of heterogeneity can clearly be identified in the pebble-bed core. Coated fuel particles within each fuel pebble form a microscopic level. A randomly packed bed of fuel pebbles mixed with moderator pebbles creates a macroscopic heterogeneity. A proper double heterogeneity modeling is a vital part of all detailed realistic studies of VHTRs.

In addition, presence of helium requires accounting for neutron streaming effects in the pebble-bed core. Phenomena associated with neutron scattering (thermalization) in graphite cannot be neglected as well.

The pebble-bed configuration represents a unique combination of coated micro-particle fuel, helium and graphite that yields flexibility in fuel selection and management and adaptability to the changing economics. Previous studies have already demonstrated that the same reactivity variation can be attained as a result of different changes of the basic pebble-bed characteristics. [3]

\section{COMPUTER CODES AND MODEL VALIDATION}

The analysis is performed using state-of-the-art computer codes that allow realistic modeling of the pebble-bed features at the particle-pebble-level as well as at the whole core level including coolant channels, control components and pebble discharge system. The list of applied codes includes SCALE 5.0, DANCOFF-MC, and MCNP $[4,5]$. The spatial self-shielding due to doubleheterogeneity effects is taken into account by using Dancoff correction factors. [5]

To confirm accuracy of the results and conclusions, the benchmark testing of the developed VHTR model is based on the actual test results and developed benchmark problems of the LEU-HTR PROTEUS Program (pebblebed criticality experiments), and the HTR-10 Program (pebble-bed core design, reactor experiments). [6] The applied VHTR model is shown in Fig. 1.

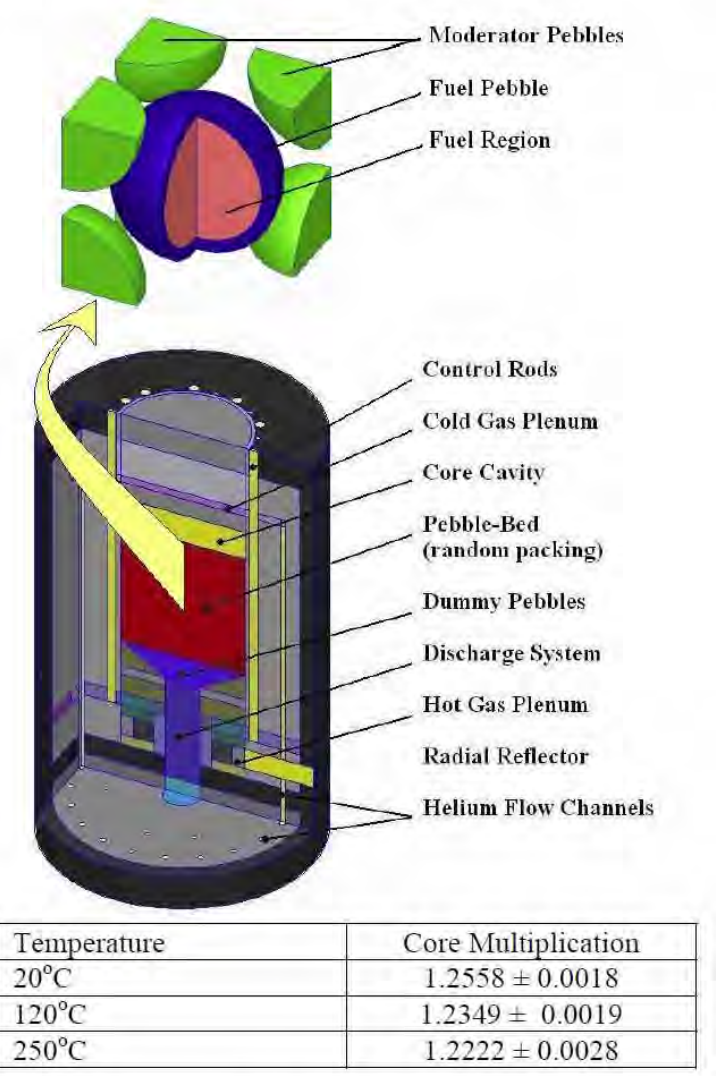

Fig. 1. Structure of the VHTR model (HTR-10 configuration, KENO3D plot).

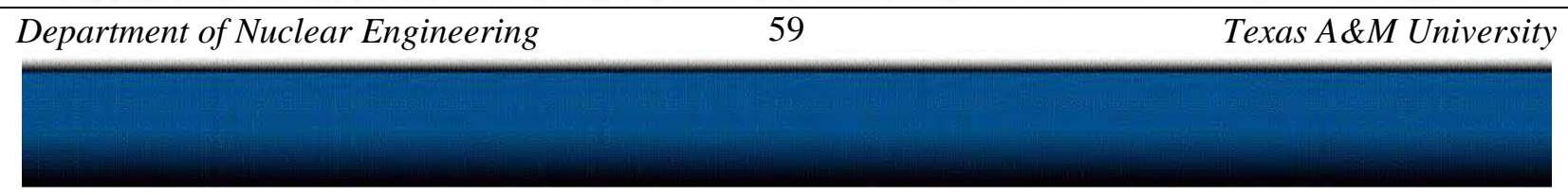




\section{Utilization of MAs as a Fuel Component for Ultra-Long Life} VHTR Configurations: Designs, Advantages and Limitations

Obtained results are consistent with the available LEU-HTR PROTEUS and HTR-10 data and confirm general applicability of the chosen modeling approach.

\section{SPECTRUM SHIFTING CAPABILITIES}

Pebble bed cores permit flexibility in component configuration. Fissile properties of minor actinides can be improved as a result of spectrum shifting through bed adjustments. Consequently, neptunium, americium and curium serve as burnable poisons or fuels facilitating small reactivity swings over prolonged irradiation periods. Figure 2 summarizes the basic actinide properties. These data are the basis for cores with postulated spectrum shifting. Figure 3 shows the estimated spectral effects due to pebble-bed variations.

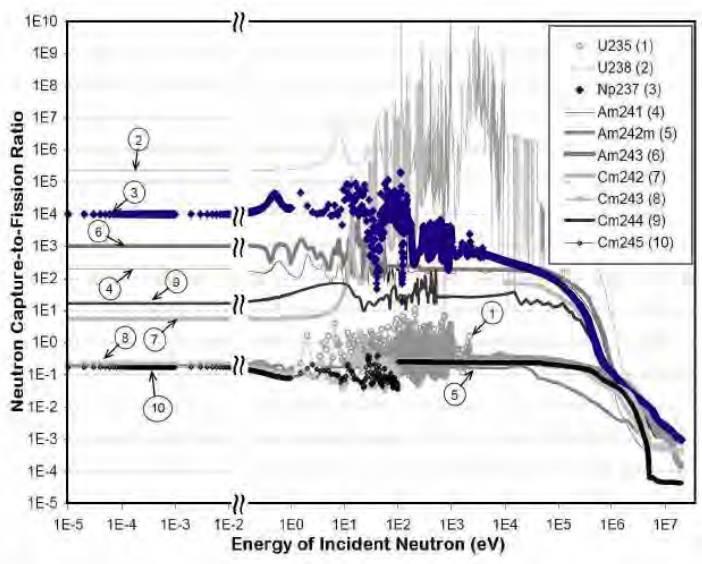

Fig. 2. Relative properties of actinide fuel components

The developed VHTR configurations are being analyzed and compared taking into account the existing systems and the Generation IV VHTR concepts.

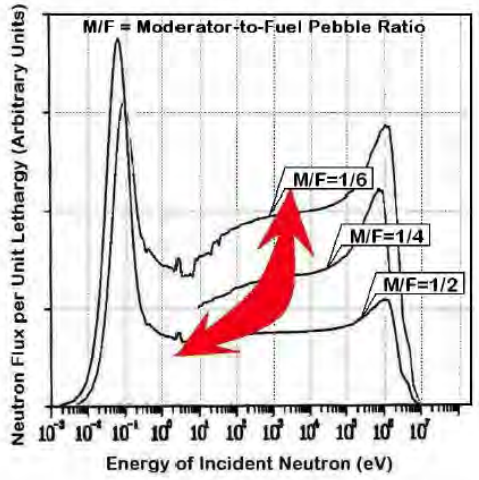

Fig. 3. Spectrum shifting in the pebble bed configurations.

\section{ACKNOWLEDGMENTS}

This paper is based upon work supported by the U.S. Department of Energy under Award Number DE-FC0705ID14655 (05-094).

\section{DISCLAIMER}

This paper was prepared as an account of work sponsored by an agency of the United States Government. Neither the United States Government nor any agency thereof, nor any of their employees, makes any warranty, express or implied, or assumes any legal liability or responsibility for the accuracy, completeness, or usefulness of any information disclosed. The views and opinions of authors expressed herein do not necessarily state or reflect those of the United States Government or any agency thereof.

\section{REFERENCES}

1. "Report to Congress on Advanced Fuel Cycle Initiative: The Future Path for Advanced Spent Fuel Treatment and Transmutation Research", 03GA50439-06, January, 2003, Office of Nuclear Energy, Science, and Technology, U.S. DOE (2003).

2. P. V. TSVETKOV, D. E. AMES II, A. B. ALAJO, "Configuration Adjustment Potential of the VHTR Prismatic Cores with Advanced Actinide Fuels", Proc. of This Conference (2006).

3. P. V. TSVETKOV, J. E. HOOGENBOOM, "Reactivity Effects in a HTGR due to Variations of Basic Pebble-Bed Characteristics", Trans. Amer. Nucl. Soc., 91, 559 (2004).

4. "SCALE: A Modular Code System for Performing Standardized Computer Analyses for Licensing Evaluation", ORNL/TM-2005/39, Ver. 5, April 2005, Oak Ridge National Laboratory, Oak Ridge, Tennessee (2005).

5. J. VALKO, P. V. TSVETKOV, J. E. HOOGENBOOM, "Calculation of the Dancoff Factor for Pebble-Bed Reactors", Nucl. Sci. Eng., 135, 304 (2000).

6. "Evaluation of High Temperature Gas-cooled Reactor Performance: Benchmark Analysis Related to Initial Testing of the HTTR and HTR-10", IAEATECDOC-1382, International Atomic Energy Agency, Vienna, Austria (2003). 


\section{Utilization of MAs as a Fuel Component for Ultra-Long Life} VHTR Configurations: Designs, Advantages and Limitations

\section{Potential of Minor Actinides to Enhance VHTR Performance Characteristics}

Pavel V. Tsvetkov and Ayodeji B. Alajo

\section{Department of Nuclear Engineering, Texas A\&M University, 129 Zachry Engineering Center, MS 3133 TAMU} CollegeStation, TX, 77843-3133,E-mail:tsvetkov@.tamu.edu

\section{INTRODUCTION}

Current recycle approach presumes actinide recycle in the conventional closed fuel cycle by reprocessing of spent LWR fuel. In this approach, recovered minor actinides are treated and stored as high level wastes because they constitute the most undesirable long-term radiotoxicity. One of the ways through which this issue may be addressed is through the use of minor actinides as possible fuel. Partitioning and transmutation of minor actinides is a very challenging option but it could have a positive impact on the global nuclear fuel cycle since it incinerates hazardous nuclides and potentially provides additional fuel supply. [1]

The objective of the U.S. DOE University NERI Project is to assess the possibility, advantages and limitations of achieving ultra-long life VHTR (Very High Temperature Reactor) configurations by utilizing minor actinides as a fuel component. The analysis takes into consideration and compares capabilities of pebble-bed and prismatic core designs with advanced actinide fuels to approach the reactor lifetime long operation without intermediate refueling.

\section{VHTR CORE FEATURES}

The VHTR system is the nearest Generation IV concept. [1] Both pebble-bed and prismatic core designs are characterized by the use of coated micro-particles as basic fuel carriers. Depending on the design, microparticles are embedded inside fuel pebbles (pebble bed core) or fuel compacts (prismatic core). This principal arrangement creates multi-heterogeneity phenomena complicating analysis of these systems. At the same time, it gives flexibility in fuel selection, management, core performance and adaptability to the changing economics. It has already been demonstrated in basic reactor physics parameter studies that the same reactivity variation can be attained as a result of different changes of the basic VHTR core characteristics. [2]

\section{VHTR MODELING METHODOLOGY}

The key technical issues are being addressed and resolved by implementing computationally efficient automated modeling procedures and sequences, combining continuous energy Monte Carlo and multigroup Monte Carlo and deterministic approaches, developing and applying realistic 3D coupled neutronicsthermal-hydraulics models with multi-heterogeneity treatments, developing and performing experimental/computational benchmarks for model verification and validation, analyzing uncertainty effects and error propagation. [3,4]

Validation data are available from power reactors as well as past critical experiments. The project uses the actual test results of the LEU-HTR PROTEUS Program (pebble-bed criticality experiments), the HTTR Program (prismatic core design, reactor experiments), and the HTR-10 Program (pebble-bed core design, reactor experiments). [5] Obtained benchmark results are in agreement with the available HTTR and HTR-10 data and confirm applicability of the chosen modeling methodology. The expected accuracy of the analysis ranges from $0.1 \%$ (selected prismatic core configurations) up to $0.45 \%$ (selected pebble-bed configurations).

\section{POTENTIAL OF MINOR ACTINIDES}

The principal mechanism being envisioned to achieve ultra-long life systems is an enhanced involvement of self-generated fissile compositions based on spent LWR fuel. Since pebble bed and prismatic core designs permit flexibility in component configuration, fuel utilization and management, it is possible to improve fissile properties of minor actinides by neutron spectrum shifting through configuration adjustments.

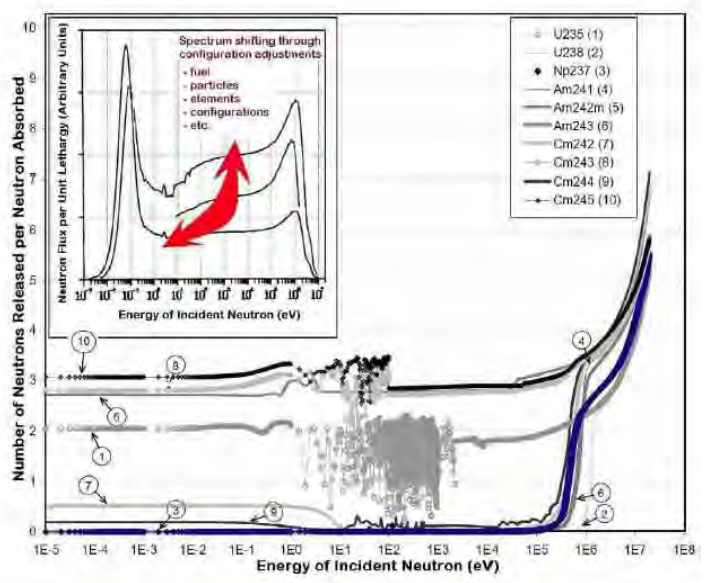

Fig. 1. Spectrum shifting and actinide neutronics.

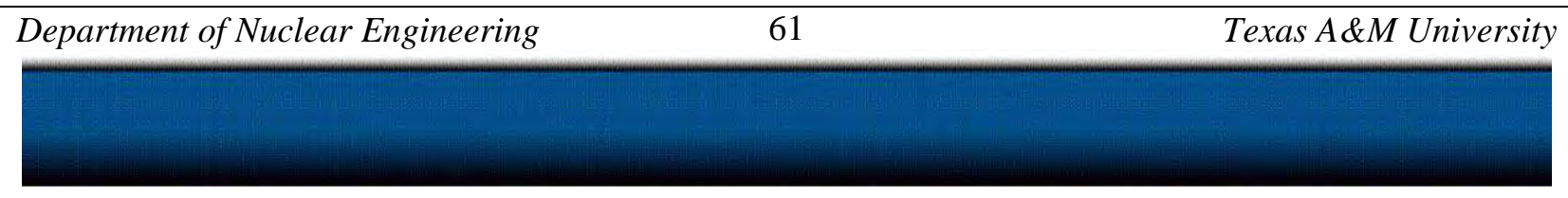




\section{Utilization of MAs as a Fuel Component for Ultra-Long Life} VHTR Configurations: Designs, Advantages and Limitations

\section{Project 05-094}

Final Scientific/Technical Report

As shown in Fig. 1, spectral effects resulting in dominant energy shift should enhance neutronics of minor actinides. Depending on neutron spectra in the considered core configurations, neptunium, americium and curium may serve as burnable poisons or fuel materials contributing to small reactivity swings (self-stabilization) over prolonged irradiation periods yielding high levels of burn-up.

However, the detailed analysis of nuclear data files shows significant variations for minor actinides. These variations cause uncertainties that may result in error propagation and erroneous conclusions. Figure 2 illustrates the effect of data variations for ${ }^{242} \mathrm{Cm}$.

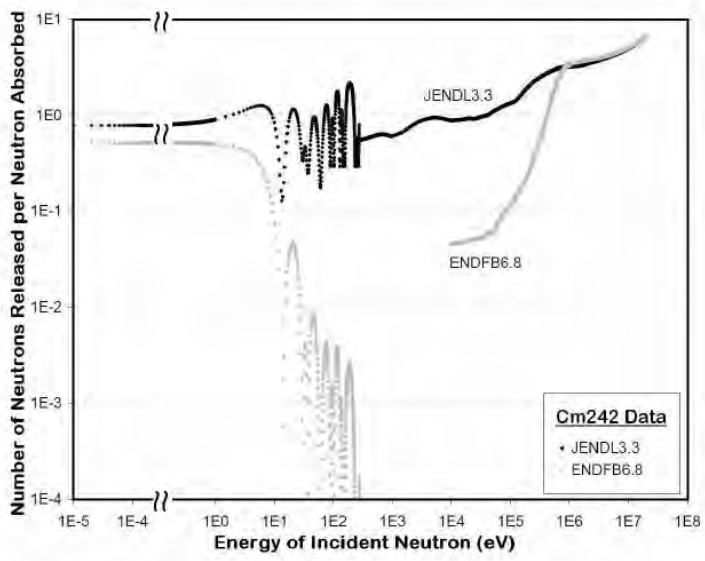

Fig. 2. Data uncertainty effect on ${ }^{242} \mathrm{Cm}$ characteristics.

Besides data uncertainties, the enhanced involvement of minor actinides is further complicated by their low delayed neutron yields. Figure 3 suggests design challenges due to dynamics and safety of the VHTRs with advanced actinide fuels.

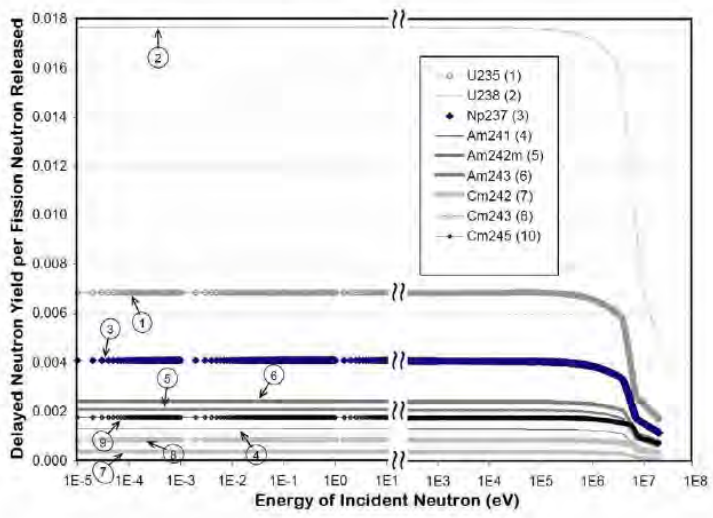

Fig. 3. Delayed neuron yield of actinide fuel components.

\section{CONCLUSIONS}

The project evaluates potential linkage and application for Generation IV VHTRs in the AFCI Program. The up-to-date analysis of the actinide-fueled VHTR configurations indicates promising performance characteristics (see Fig. 1) as well as potential areas of concern (see Fig. 2) and further design refinement needs (see Fig. 3).

\section{ACKNOWLEDGMENTS}

This paper is based upon work supported by the U.S Department of Energy under Award Number DE-FC0705ID14655 (05-094)

\section{DISCLAIMER}

This paper was prepared as an account of work sponsored by an agency of the United States Government. Neither the United States Government nor any agency thereof, nor any of their employees, makes any warranty, express or implied, or assumes any legal liability or responsibility for the accuracy, completeness, or usefulness of any information disclosed. The views and opinions of authors expressed herein do not necessarily state or reflect those of the United States Government or any agency thereof.

\section{REFERENCES}

1. "Report to Congress on Advanced Fuel Cycle Initiative: The Future Path for Advanced Spent Fuel Treatment and Transmutation Research", 03GA50439-06, January, 2003, Office of Nuclear Energy, Science, and Technology, U.S. DOE (2003).

2. P. V. TSVETKOV, J. E. HOOGENBOOM, "Reactivity Effects in a HTGR due to Variations of Basic Pebble-Bed Characteristics", Trans. Amer. Nucl. Soc., 91, 559 (2004).

3. "SCALE: A Modular Code System for Performing Standardized Computer Analyses for Licensing Evaluation", ORNL/TM-2005/39, Ver. 5, April 2005, Oak Ridge National Laboratory, Oak Ridge, Tennessee (2005).

4. J. VALKO, P. V. TSVETKOV, J. E. HOOGENBOOM, "Calculation of the Dancoff Factor for Pebble-Bed Reactors", Nucl. Sci. Eng., 135, 304 (2000).

5. "Evaluation of High Temperature Gas-cooled Reactor Performance: Benchmark Analysis Related to Initial Testing of the HTTR and HTR-10", IAEATECDOC-1382, International Atomic Energy Agency, Vienna, Austria (2003). 


\section{VHTR PERFORMANCE ANALYSIS}

The VHTR multi-heterogeneity features allow material separation by using different types of micro-particles and graphite blocks. The degree of heterogeneity and the moderator-to-fuel ratio can be adjusted to achieve desirable spectrum shifting. As illustrated, depending on dominant neutron energies in the considered core configurations, neptunium, americium and curium may serve not only as burnable poisons (high absorption (capture) but low neutron yield) but also as high-yield fuel materials contributing to self-stabilization effects that allow prolonged single-loading lifetimes.

The whole-core models with multi-heterogeneity treatments and property databases of actinide compounds have been developed together with relevant benchmark problems to compare computational results with experimental data. Code-to-code and experiment-to-code studies of the models and computational schemes are underway as well as analysis of the actinide-fueled VHTR configurations. Specifically, several technical challenges are associated with the analysis and development of ultra-long life VHTR configurations with minor actinides as a fuel component:

- whole core/system modeling with multi-heterogeneity treatments,

- model benchmarking, uncertainty effects of nuclear data and design parameters including temperature dependence, graphite impurities, and etc.

- error propagation during depletion calculations,

- large computational times affecting ability to consider many whole-core design configurations.

In the present study, state-of-the-art computer code systems are utilized to create realistic modeling of the VHTR configurations. The created VHTR model is a nearly explicit representation of the existing core configurations.

A special effort is made to verify that the computational modeling is consistent and realistic. The results describe performance of the entire VHTR power unit and allow conclusions regarding the configuration's feasibility, performance and possible directions for further analysis and development. Using 3D whole-core/system models, researchers developed a variation analysis methodology and then will apply it for studies of VHTR geometry and material variations. The existing HTTR and HTR-10 configurations served as the initial prototype designs and as examples of small-scale VHTRs. Figure 13 illustrates the planned basic parameters variations using the HTR-10 pebble bed core as an example. 
Dimensions and geometry

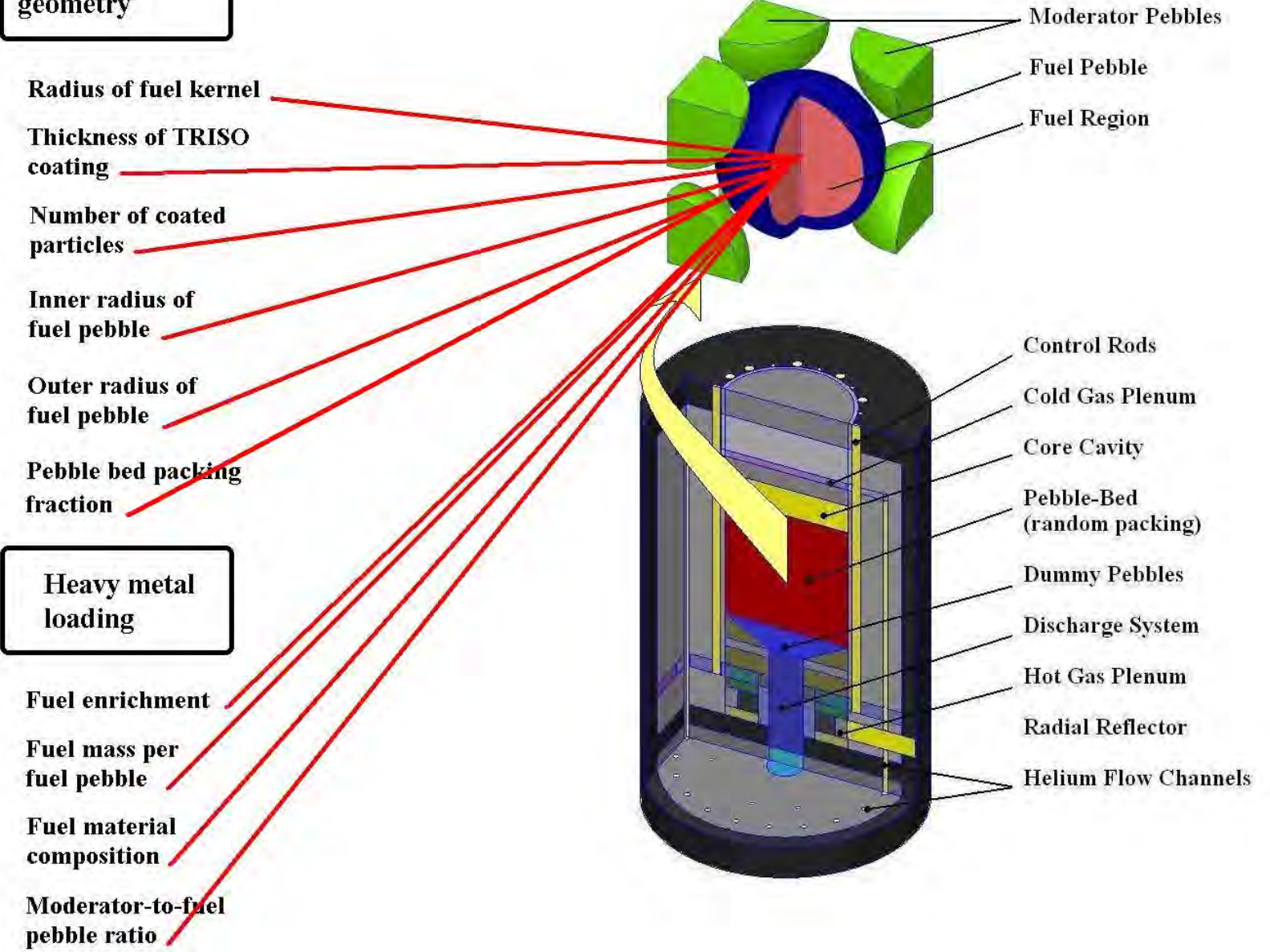

Figure 13. Basic pebble bed parameters. 


\title{
SPECTRUM SHIFTING AS A MECHANISM TO IMPROVE PERFORMANCE OF VHTRS WITH ADVANCED ACTINIDE FUELS
}

\author{
Pavel V. Tsvetkov, David E. Ames, Megan L. Pritchard, Ayodeji B. Alajo \\ Department of Nuclear Engineering \\ Texas A\&M University \\ 129 Zachry Engineering Center, MS 3133 TAMU, College Station, Texas 77843-3133, USA \\ Phone: $979 / 845-7078$, \\ Fax: 979/845-6443 \\ E-mail:Tsvetkov@tamu.edu
}

\section{KEYWORDS}

Minor actinides, VHTR, recycle, closed fuel cycle

\begin{abstract}
Reprocessing of spent LWR fuel is an intrinsic part of the closed fuel cycle. While current technologies treat recovered minor actinides as high level wastes, the primary objective of one of the U.S. DOE Nuclear Energy Research Initiative (NERI) projects is to assess the possibility, advantages and limitations of achieving ultra-long life VHTR (Very High Temperature Reactor) configurations by utilizing minor actinides as a fuel component. The postulated principal mechanism is an enhanced involvement of self-generated fissile compositions based on spent LWR fuel. Since pebble bed and prismatic core designs permit flexibility in component configuration, fuel utilization and management, it is possible to improve fissile properties of minor actinides by neutron spectrum shifting through configuration adjustments. Depending on neutron spectra, neptunium, americium and curium may contribute to small reactivity swings (selfstabilization) over prolonged irradiation periods. The presented analysis is focused on achievability of spectral variations and their potential impact. In principle, promising core features and performance characteristics have been demonstrated.
\end{abstract}

\section{INTRODUCTION}

Partitioning and transmutation (P\&T) of minor actinides (MA) are expected to have a positive impact on the future of nuclear technology. Recovered MAs are currently managed as high level wastes (HLW). [1] Implementation of P\&T would lead to incineration of hazardous nuclides and could potentially provide additional fuel supply.
The VHTR system is one of the promising Generation IV reactor concepts. [2] The proposed earlier by researchers at General Atomics, LANL, and ORNL, Deep-Burn transmutation approach suggests thermal neutron transmutation of MAs in the HTGR prismatic cores taking advantage of actinide nuclear properties. [3] The primary objective of the present U.S. DOE NERI project is to assess the possibility, advantages, and limitations of achieving ultra-long life VHTR configurations by utilizing minor actinides as a fuel component. The project intrinsically results in optimized transmutation of MAs but, instead of attaining high burnup levels, it focuses on the complete recovery of the energy value of MAs. Since pebble bed and prismatic core designs permit flexibility in component configuration, fuel utilization and management, it should be possible to improve fissile properties of MAs by neutron spectrum shifting through configuration adjustments. Depending on neutron spectra, neptunium, americium and curium may contribute to small reactivity swings (selfstabilization) over prolonged irradiation periods. As a result, under certain spectral conditions MAs would be able to contribute to the core neutron balance compensating for fuel depletion effects through their chain transformations. The resulting self-stabilization of advanced actinide fuels should allow prolonged operation on a single fuel loading up to ultralong lifetimes that are comparable with the projected entire reactor lifetime of $40-60$ years limited by structural components performance due to thermal loads, mechanical stresses and attained fluences (up to the lifetime of a power unit).

The VHTR systems with MAs are developed and analyzed accounting for controllability, dynamics and safety margins, and proliferation-resistance during the entire reactor lifetime.

\begin{tabular}{lll}
\hline Department of Nuclear Engineering & 65 & Texas A\&M University \\
\hline
\end{tabular}




\section{Utilization of MAs as a Fuel Component for Ultra-Long Life} VHTR Configurations: Designs, Advantages and Limitations

\section{Project 05-094}

\section{Final Scientific/Technical Report}

The resulting configurations should have inherent capabilities for utilization of MAs from spent LWR fuel, reduction of spent fuel flows and handling per unit of produced energy, and potential for autonomous operation with minimized maintenance. Their broad deployment would allow reducing the long-term radiotoxicity and heat load of HLW sent to a geologic repository and enable recovery of the remaining energy value of spent fuel compositions.

Utilization of MAs as a fuel component would facilitate development of new fuel cycles and support sustainability of a fuel source for nuclear energy, assuring future operation of Generation IV nuclear energy systems. The ultra-long core life approach reduces the technical need for additional repository. Furthermore, the developed VHTR configurations should improve marketability of the Generation IV VHTRs intended for hydrogen production because their implementation allows worldwide deployment including developing countries and remote areas

The presented analysis is focused on achievability of spectral variations and their potential impact. In principle, promising core features and performance characteristics have been demonstrated. The postulated principal mechanism is an enhanced involvement of self-generated fissile compositions based on spent LWR fuel.

\section{NOMENCLATURE \\ AFCI - Advanced Fuel Cycle Initiative \\ HLW - High Level Waste \\ HTGR - High Temperature Gas-cooled Reactor \\ LANL - Los Alamos National Laboratory \\ LEU - Low-Enriched Uranium \\ MA - Minor Actinides (Np, Am, Cm) \\ NERI - Nuclear Energy Research Initiative \\ ORNL - Oak Ridge National Laboratory \\ P\&T - Partitioning and Transmutation \\ $\mathrm{RPu} \quad-$ Reactor-grade Plutonium \\ TRU - Transuranium nuclides \\ VHTR - Very High Temperature Reactor}

\section{MODELING APPROACH}

Reliable performance prediction is a challenging endeavor for advanced nuclear energy systems like Generation IV VHTRs due to their inherent safety features resulting in tight coupling between energy production, transport and conversion, and system behavior during normal operation and off-normal situations. To create advanced nuclear energy systems it is desirable to have a modeling-based design development that relies on simulating features of the entire life cycle of the system before actual physical prototyping - from concept development to detailed design, prototyping, and safety analysis. This approach should provide consistency between neutronics and other performance aspects such as thermal hydraulics, mechanical integrity, and plant heat transfer and conversion balances.
The suggested integrated/hybrid Monte-Carlodeterministic modeling approach offers the desirable analysis capabilities for the VHTR design studies. It was originally developed and successfully used for fission fragment magnetic collimator reactors. [4]

Within the computational scheme, key technical issues are being addressed and resolved by:

- implementing efficient automated modeling procedures and sequences,

- combining Monte Carlo and deterministic approaches,

- developing and applying realistic 3D coupled neutronics-thermal-hydraulics models with multiheterogeneity treatments,

- developing and performing experimental/computational benchmarks for model verification and validation,

- analyzing uncertainty effects and error propagation

The use of Monte Carlo codes creates a potential for explicit 3D whole-core/reactor studies of the VHTR configurations addressing the key reactor physics areas such as the double and multi-level heterogeneity, neutron streaming in the low-density regions, and neutron spectrum transitions at the interfaces. Coupling approach is important for modeling of the dynamic behavior and assessment of inherent safety. Space and burnup dependent reactivity coefficients and feedback analysis have paramount importance for inherent safety confirmation.

The computational scheme of the analysis of actinidefueled VHTRs is shown in Fig. 1. The VHTR modeling is based on and is supported by the following computer code systems: MCNP, SCALE, HeatWave, Dancoff-MC, DENT 97, and general-purpose software packages for applied mathematics and graphics. [5-9]

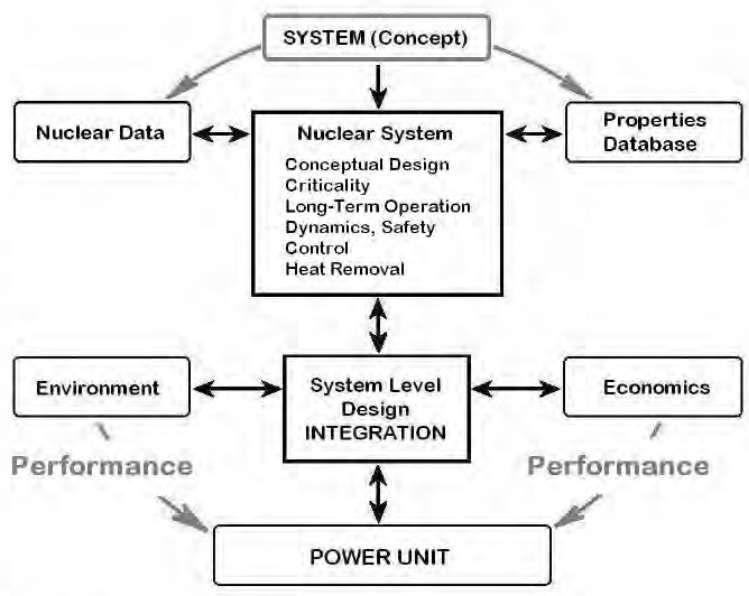

Figure 1. Applied system analysis sequence. 


\section{Utilization of MAs as a Fuel Component for Ultra-Long Life VHTR Configurations: Designs, Advantages and Limitations}

\section{Project 05-094}

Final Scientific/Technical Report
The presented scheme is followed as an overall design procedure for the VHTR system up to a power unit level. It combines the available capabilities of the SCALE Monte Carlo (whole-core analysis level) and deterministic functional modules (lattice level) with the HeatWave finite element solver. The resonance self-shielding calculations are performed accounting for double heterogeneity effects by so-called Dancoff correction factors. [8] The Dancoff-based approach has successfully been implemented for and used in many parametric studies of VHTRs. [8,10-12] The analysis confirms earlier observations that, although double heterogeneity effects are less pronounced in prismatic cores than in pebble-bed cores, the estimated overall sensitivity span is up to $6 \%$ per factor of 4 in varying Dancoff correction factors, $[10,13]$

The adequacy of the applied computational methods and models used to evaluate performance is assessed by comparisons with experimental data covering appropriate ranges of conditions. Validation data are available from research reactors and zero-power critical assemblies. The project uses the actual test results and developed benchmark problems of the LEU-HTR PROTEUS Program (pebble-bed criticality experiments), the HTTR Program (prismatic core design, reactor experiments), and the HTR-10 Program (pebble-bed core design, reactor experiments). [14-16] Observed differences are taken into account at the implementation and interpretation stages of the present analysis.

Table 1 illustrates the observed level of agreement with experimental data. The modeling results are obtained using general material properties and standard SCALE cross section libraries without any special tailoring to specific systems.

Table 1. Code-to-Experiment Validation of the VHTR Model

\begin{tabular}{|c|c|}
\hline Benchmark & $\begin{array}{l}\text { Code-to-Test } \\
\text { Discrepancy } \\
(\%)\end{array}$ \\
\hline $\begin{array}{l}\text { Prismatic Core: } \\
\text { - } k_{\text {eff }}, \text { Control Rods Out } \\
\text { - } k_{\text {eff }}, \text { Control Rods Fully In } \\
\text { - Control Rod Insertion, } 300 \mathrm{~K} \\
\text { - Control Rod Insertion, } 418 \mathrm{~K} \\
\text { - } \\
\alpha_{T}\end{array}$ & $\begin{array}{l}-1.20 \\
+5.10 \\
+2.30 \\
+2.80 \\
+13.0\end{array}$ \\
\hline $\begin{array}{l}\text { Pebble-Bed Core: } \\
\text { - } k_{\text {eff }} \text {, Control Rods Out, } 293 \mathrm{~K} \\
\text { - } k_{\text {eff }} \text {, Control Rods Out, } 393 \mathrm{~K} \\
\text { - } k_{\text {eff }} \text {, Control Rods Out, } 523 \mathrm{~K}\end{array}$ & $\begin{array}{l}+11.0 \\
+9.70 \\
+10.0\end{array}$ \\
\hline
\end{tabular}

The code-to-experiment discrepancy of about $10 \%$ is generally targeted as successful validation of a particular code applicability. [16] The observed larger deviation of computational results from experimental values for the cores with inserted control rods is due to limited representation of control systems in the VHTR models. The computed value of the isothermal temperature coefficient, $\alpha_{T}$, deviates by $13 \%$ from the corresponding experimental value. However, the experimental value is within the standard deviation limits of the computational result.

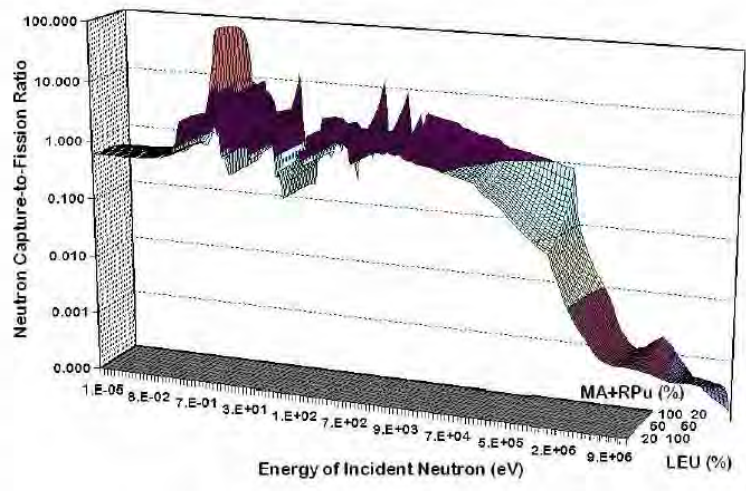

(a) Neutron-induced fission potential

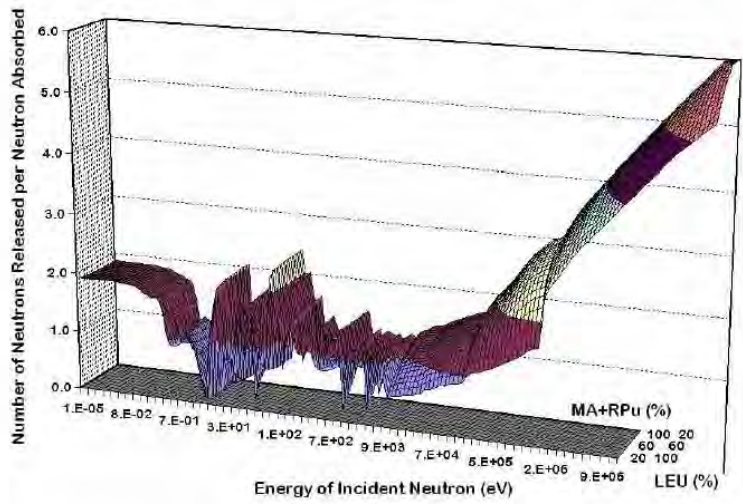

(b) Neutron reproduction factor

Figure 2. Nuclear performance characteristics of the advanced actinide mixtures (MA-RPu-LEU).

\section{MINOR ACTINIDES, PLUTONIUM AND LEU}

Figure 2 compares fundamental nuclear performance characteristics of advanced actinide mixtures consisting of MAs, reactor-grade plutonium (RPu) and low-enriched uranium (LEU). To estimate an optimum fissile content and to identify favorable spectral conditions in the VHTR system, energy-composition dependent characteristics of the advanced actinide mixtures have to be analyzed as "optimization 


\title{
Utilization of MAs as a Fuel Component for Ultra-Long Life
} VHTR Configurations: Designs, Advantages and Limitations

\section{Project 05-094}

\author{
Final Scientific/Technical Report
}

surfaces". Based on the surface plots shown in Fig. 2, mixtures with high content of MAs and RPu (high content of TRUs) should be considered as the most promising candidates. It can also be concluded that the ultra-long life VHTR configurations with MAs are anticipated to have intermediate neutron energies as dominant.

\section{VHTR PERFORMANCE ANALYSIS}

The VHTR core has features contributing to fundamental differences in reactor physics of this system in comparison with other reactor concepts. The use of coated micro-particles creates multi-heterogeneity that defines performance characteristics of the VHTR core and complicates studies of these systems. At the same time, it gives flexibility in fuel selection, management, core performance and adaptability. It has already been demonstrated in basic reactor physics parameter studies that the same reactivity variation can be attained as a result of different changes in the basic VHTR core characteristics. [10]

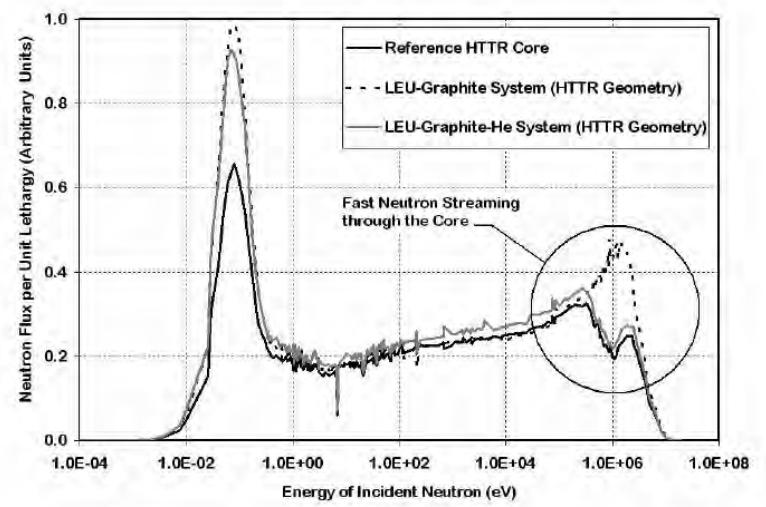

Figure 3. Neutron streaming effects - compact-average flux behavior in the VHTR prismatic core configurations.

The first level of heterogeneity (micro-level) is formed by coated fuel micro-particles that are randomly distributed in the fuel region of the graphite fuel pebble (pebble-bed core) or in the graphite compact (prismatic graphite block core). The second level of heterogeneity (macro-level) is formed by the randomly-packed bed of pebbles or by the prismatic blocks with compacts that compose the VHTR core, respectively. For desirable performance characteristics, the pebble-bed core may also contain some number of dummy moderator pebbles to achieve the required moderator-to-fuel ratio. The prismatic graphite block core has channels for coolant, instrumentation and handling operations. As a result, there are various neutron streaming passages formed by pebble-bed voids, coolant channels, instrumentation and handling channels, plenums, and etc. The proximity to outer and central reflector regions is also an important factor that influences the VHTR physics. These features act and can be taken into account as multiple additional levels of heterogeneity.

The resonance self-shielding effects are determined by the microscopic flux behavior in the fuel, which strongly depends on the geometry of the fuel configuration. Presence of helium requires accounting for neutron streaming effects. Effects of fast neutron streaming through the VHTR prismatic core are shown Fig. 3. It is apparent that the multi-heterogeneity effects should be expected to play a defining role.

Significant flexibility in component configuration, fuel utilization and management is one of the characteristic features of the VHTR design including both, pebble-bed and prismatic core configurations. The VHTR multi-heterogeneity features allow material separation by using different types of microparticles and graphite blocks. The degree of heterogeneity and the moderator-to-fuel ratio can be adjusted to achieve desirable spectrum shifting.

Thus, it should be possible to enhance neutron reaction properties of MAs by neutron spectrum shifting through configuration adjustments. Anticipated spectral variations are shown in Fig. 4. Depending on neutron spectra in the considered core configurations (dominant neutron energies), neptunium, americium and curium may serve as burnable poisons or fuel materials contributing to small reactivity swings (self-stabilization) over prolonged irradiation periods up to the entire reactor lifetime.

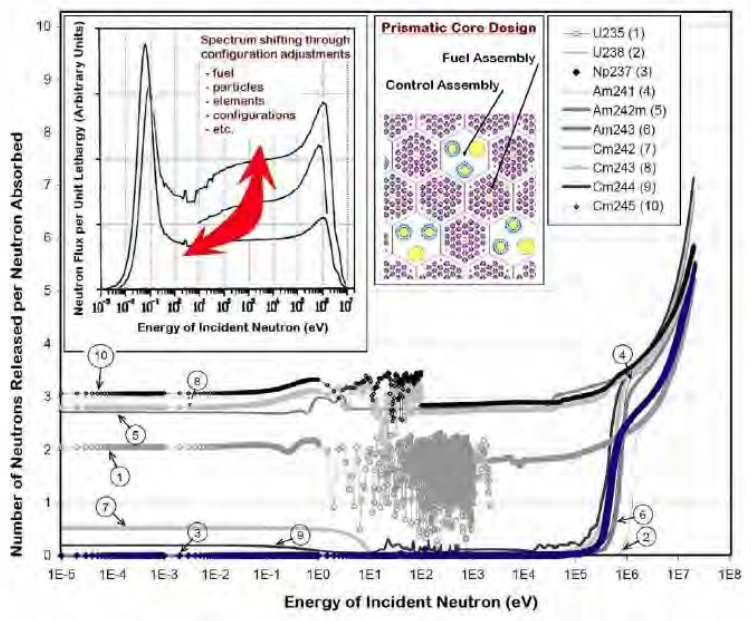

Figure 4. Spectral variations and neutron reproduction.

However, the detailed analysis of nuclear data files shows significant variations for MAs and TRUs, in general. These variations cause uncertainties that may result in error propagation and erroneous conclusions.

Besides data uncertainties, the enhanced involvement of MAs is further complicated by their low delayed neutron 


\section{Utilization of MAs as a Fuel Component for Ultra-Long Life} VHTR Configurations: Designs, Advantages and Limitations

\section{Project 05-094}

Final Scientific/Technical Report yields. Significant variations of TRU delayed neutron yields per fission neutron released are shown in Fig. 5. Delayed neutron yields for some of TRUs are several orders of magnitude lower than for ${ }^{238} \mathrm{U}$. These variations suggest design challenges due to dynamics and safety of the VHTRs with advanced actinide fuels. The VHTR system with MAs or TRUs, in general, will have dynamics characteristics that are very similar to fast reactors.

\section{CONCLUSIONS}

The project has entered its second year of studies. Wholecore/system 3D models with multi-heterogeneity treatments and properties databases of actinide compounds have been developed and implemented. Studies of the actinide-fueled VHTR configurations are underway. As presented in this paper, the analysis indicates promising performance. Depending on dominant neutron energies in the considered core configurations, neptunium, americium and curium may serve not only as burnable poisons (high absorption (capture) but low neutron yield) but also as high-yield fuel materials contributing to self-stabilization effects that allow prolonged single-loading lifetimes. The results are being analyzed and compared taking into account the existing systems and the Generation IV VHTR concepts with estimated characteristics

Observed design challenges include low delayed neutron yields for some of TRU's and their nuclear data uncertainties. It is anticipated that ultra-long life VHTR cores with MAs or TRUs will have intermediate neutron energies as dominant.

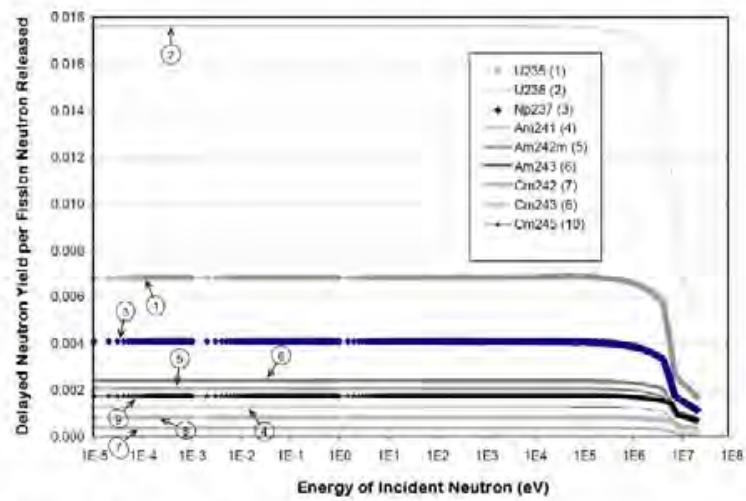

Figure 5. Spectral variations and neutron reproduction.

\section{ACKNOWLEDGMENTS}

This paper is based upon work supported by the U.S. Department of Energy under Award Number DE-FC0705ID14655 (05-094).

\section{DISCLAIMER}

This paper was prepared as an account of work sponsored by an agency of the United States Government. Neither the United States Government nor any agency thereof, nor any of their employees, makes any warranty, express or implied, or assumes any legal liability or responsibility for the accuracy. completeness, or usefulness of any information. apparatus, product, or process disclosed, or represents that its use would not infringe privately owned rights. Reference herein to any specific commercial product, process, or service by trade name, trademark, manufacturer, or otherwise does not necessarily constitute or imply its endorsement, recommendation, or favoring by the United States Govermment or any agency thereof. The views and opinions of authors expressed herein do not necessarily state or reflect those of the United States Government or any agency thereof.

\section{REFERENCES}

[1] United States Department of Energy, Office of Nuclear Energy. Science, and Technology, 2003, "Report to Congress on Advanced Fuel Cycle Initiative: The Future Path for Advanced Spent Fuel Treatment and Transmutation Research", 03-GA50439-06, U.S. DOE.

[2] United States Department of Energy, Office of Nuclear Energy, Science, and Technology, 2005, "Generation IV Nuclear Energy Systems: Ten Year Program Plan", March 2005, Office of Advanced Nuclear Research, U.S. DOE.

[3] C. Rodriguez, A. Baxter, D. McEachern, M. Fikani, F. Venneri, 2003, "Deep-Burn: Making Nuclear Waste Transmutation Practical", Nucl. Eng. Design, 2805, I.

[4] P. V. Tsvetkov, R. R. Hart, 2005, "Hybrid Monte CarloDeterministic Analysis of Fission Fragment Magnetic Collimator Reactors", Proc. Intern. Conf. on Monte Carlo Methods, Chattanooga, Temessee, April 17-21. 2005. Amer. Nucl. Soc, Lagrange Park, IL.

[5] J. F. Briesmeister, ed., 2003, "MCNP. A General Monte Carlo N-Particle Transport Code, Version 5, User's Manual", LA-UR-03-1987, Los Alanos National Laboratory.

[6] Oak Ridge National Laboratory, 2005, "SCALE: a Modular Code System for Performing Standardized Computer Analyses for Licensing Evaluation", ORNL/TM-2005/39, ORNL.

[7] S. Humphries, 1997, Field Solutions on Computers, CRC Press, Boca Raton. Florida. 


\section{Utilization of MAs as a Fuel Component for Ultra-Long Life} VHTR Configurations: Designs, Advantages and Limitations

[8] J. Valko, P. V. Tsvetkov, J. E. Hoogenboom, 2000, "Calculation of the Dancoff Factor for Pebble-Bed Reactors", Nucl. Sci. Eng., 135, 304.

[9] P. V. Tsvetkov, A. N. Chmelev, N. I. Geraskin, 1998, "Complex Multi-Task Analysis of the Nuclear Reactor Systems with the DENT-97 Computer Code System", Proc. Intern. Conf. on the Phys. of Nucl. Sci. and Tech., Long Island, New York, October 5 - 8, 1998, 2, 851, Amer. Nucl. Soc., Lagrange Park, IL.

[10]P. V. Tsvetkov, 1997, "Basic Reactor Physics Parameter Study of the Pebble-Bed Nuclear Reactor System", IRI131-97-002, Jan., 1997, TUDelft, the Netherlands.

[11]A. M. Ougouag, H. D. Gougar, W. K. Terry, R. Mphahlele, K. N. Ivanov, 2004, "Optimal Moderation in the PebbleBed Reactor for Enhanced Passive Safety and Improved Fuel Utilization", Proc. PHYSOR 2004, Chicago, Illinois, April 25-29, 2004, Amer. Nucl. Soc., Lagrange Park, IL.

[12]P. V. Tsvetkoy, J. E. Hoogenboom, 2004, "Reactivity Effects in a HTGR due to Variations of Basic Pebble-Bed Characteristics", Trans. Amer. Nucl. Soc., 91, 559.

[13]R. Plukiene, D. Ridikas, 2003, "Modeling of HTRs with Monte Carlo: from a Homogeneous to an Exact Heterogeneous Core with Microparticles", An. Nucl. En., 30, 1573 .

[14]R. Chawla, D. Mathews, 1990, "LEU-HTR PROTEUS Calculational Benchmark Specifications", TM-41-90-32, PSI, Villigen, Switzerland.

[15] International Atomic Energy Agency, 2001, "Critical Experiments and Reactor Physics Calculations for LowEnriched HTGRs", IAEA-TECDOC-1249, IAEA, Vienna, Austria.

[16] International Atomic Energy Agency, 2003, "Evaluation of High Temperature Gas-Cooled Reactor Performance: Benchmark Analysis Related to Initial Testing of the HTTR and HTR-10", IAEA-TECDOC-1382, IAEA, Vienna, Austria. 


\section{Neutronics Analysis of Pebble-Bed Cores with TRUs}

Megan L. Pritchard, Pavel V. Tsvetkov, Ayodeji B. Alajo

Department of Nuclear Engineering, Texas A\&M University, 129 Zachry Engineering Center, MS 3133 TAMU

College Station, TX, 77843-3133,E-mail: tsvetkov@.tamu.edu

\section{INTRODUCTION}

The Next Generation Nuclear Plant (NGNP) concept envisions an advanced, efficient, next-generation nuclear reactor designed for electricity generation and hydrogen production. One of the six Generation IV concepts, Very High Temperature Reactor (VHTR), is specifically intended for this application but could also serve as a high temperature heat source for other industrial applications like sea water desalination and various petrochemical applications. The VHTR design originates from High Temperature Gas Cooled Reactors (HTGR) which have successfully demonstrated their feasibility. The VHTR concept has been proposed in two design configurations: a prismatic core consisting of fuel and graphite blocks, and a pebble-bed core composed of thousands of fuel and graphite spheres. Prototypes of each of these configurations exist as the High Temperature Test Reactor (HTTR) in Japan and the Chinese High Temperature Test Module (HTR-10) in China. [1] The NGNP/VHTR technology is expected to enter commercial energy markets after 2030 .

Apart from hydrogen production, the VHTR technology is being seriously considered as one of the thermal neutron transmutation approaches for transuranic (TRU) nuclides incineration. Researchers at General Atomics, LANL, and ORNL proposed a so-called DeepBurn transmutation in the HTGR prismatic cores. [2] The U.S. DOE NERI project considers TRU vectors as potential fuel components that could enhance performance characteristics of power units with VHTRs by prolonging their operation on a single fuel loading. The project inherently considers optimized transmutation of TRUs by completely recovering their energy value. This paper discusses the studies focused on pebble-bed cores with TRUs.

\section{PEBBLE-BED CORES AND THEIR MODELING}

The principal mechanism to achieve ultra-long life VHTR configurations is an enhanced involvement of selfgenerated fissile compositions based on a spent LWR fuel. The pebble beds offer many incentives for core configuration flexibility which can lead to improved fissile properties of TRUs by neutron spectrum shifting. For example, by altering the moderator pebble to fuel pebble ratio, total number of pebbles or size of the pebbles, various spectral conditions can be achieved within the same core. Evidently, the uncertainties associated with nuclear data and VHTR design characteristics are imperative in understanding the modeling reliability and in determining plausible designs.

The analysis is performed at the whole core level properly accounting for multi-heterogeneity phenomena due micro-particles, pebbles and gaseous coolant. The applied state-of-the-art computer codes allow fine-scale modeling of the pebble-bed features at the particlepebble-level as well as at the whole core level including coolant channels, control components and pebble discharge systems. The ORNL SCALE code system is the primary tool of this research effort. [3] The performed model testing is based on the experiment-to-code benchmarks of the LEU-HTR PROTEUS Program (pebble-bed criticality experiments), and the HTR-10 Program (pebble-bed core design, reactor experiments). [1]

The considered pebble-bed configurations are shown in Fig. 1. Both, small-scale and large-scale, power reactor options have been taken into account.

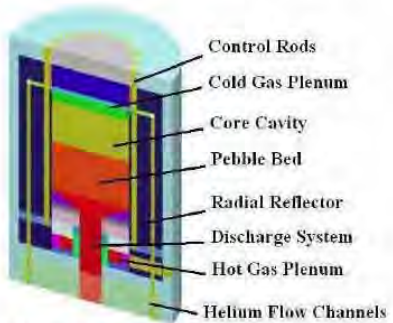

(a) Small-Scale Power Unit

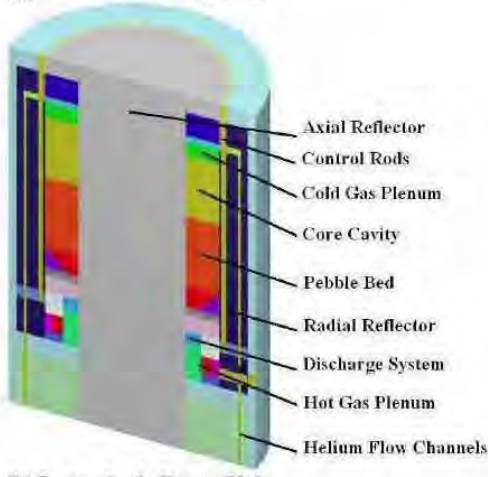

(b) Large-Scale Power UTnit

Fig. 1. Small-scale and large scale pebble bed cores (KENO3D plot).

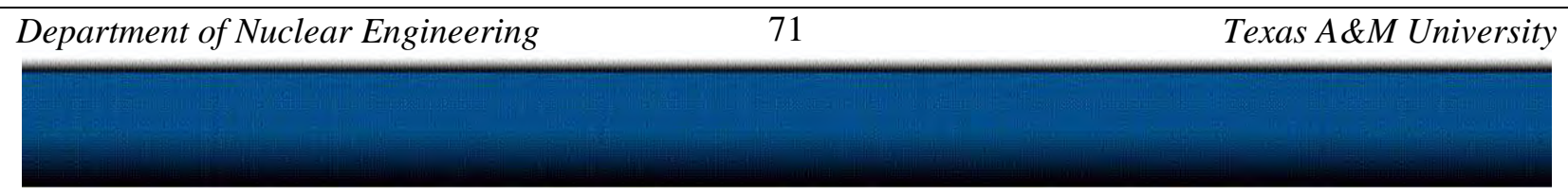




\section{Utilization of MAs as a Fuel Component for Ultra-Long Life} VHTR Configurations: Designs, Advantages and Limitations

\section{Project 05-094}

\section{Final Scientific/Technical Report}

\section{RESULTS - TRU-FUELED PEBBLE-BED CORES}

To estimate an optimum fissile content and to identify favorable spectral conditions in the system, energy-composition dependent characteristics have to be analyzed. Figure 2 shows neutronics performance of minor actinides (MA) mixed with LEU (3.7\%).

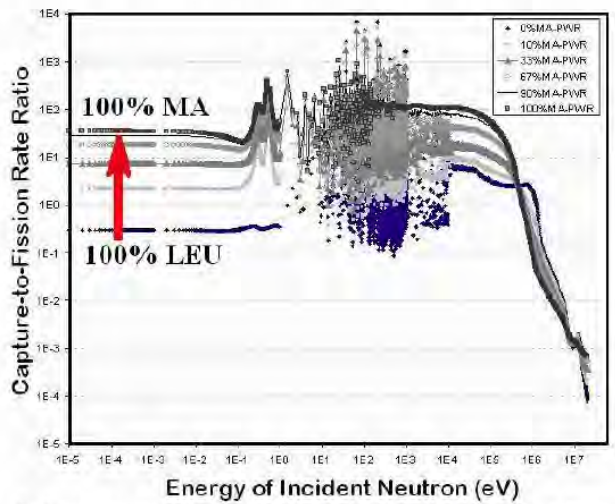

Fig. 2. Neutronics characteristics of MA-LEU mixtures

Depending on dominant neutron energies in the considered core configurations, MAs may have high neutron yields or act as absorbers. The observations are not completely conclusive due to uncertainties in nuclear data.

As illustrated in Table $I$, specific spectral characteristics can be varied in the pebble bed core by mixing graphite pebbles and fuel pebbles. This observation is consistent with the earlier conclusions. [4] The degree of heterogeneity and the moderator-to-fuel ratio can be adjusted to achieve the desirable spectrum shifting.

TABLE I. Moderator-to-Fuel Pebble Ratio Variations

\begin{tabular}{|l|l|l|l|}
\hline$T_{\text {CORE }}(\mathbf{K})$ & $\begin{array}{l}\text { M/F Pebble } \\
\text { Ratio }\end{array}$ & Reactivity (\%) & $\begin{array}{l}\text { Energy Inducing } \\
\text { Fission (eV) }\end{array}$ \\
\hline 300 & $0 / 1$ & +5.740 & $0.0722 \pm 0.0004$ \\
\cline { 2 - 4 } & $1 / 3$ & +2.420 & $0.0619 \pm 0.0004$ \\
\cline { 2 - 4 } & $2 / 1$ & -19.674 & $0.0450 \pm 0.0003$ \\
\hline 393 & $0 / 1$ & +5.321 & $0.0837 \pm 0.0002$ \\
\cline { 2 - 4 } & $1 / 3$ & +0.537 & $0.0717 \pm 0.0002$ \\
\cline { 2 - 4 } & $2 / 1$ & -22.429 & $0.0535 \pm 0.0001$ \\
\hline 523 & $0 / 1$ & +4.306 & $0.1000 \pm 0.0002$ \\
\cline { 2 - 4 } & $1 / 3$ & -0.402 & $0.0871 \pm 0.0002$ \\
\cline { 2 - 4 } & $2 / 1$ & -25.691 & $0.0670 \pm 0.0001$ \\
\hline \multirow{3}{*}{1000} & $0 / 1$ & +0.299 & $0.1629 \pm 0.0003$ \\
\cline { 2 - 4 } & $1 / 3$ & -5.274 & $0.1461 \pm 0.0002$ \\
\cline { 2 - 4 } & $2 / 1$ & -37.061 & $0.1189 \pm 0.0002$ \\
\hline \multirow{3}{*}{} & & & \\
\hline
\end{tabular}

The average energy-dependent fluxes for $\mathrm{UO}_{2}, \mathrm{UC}$, TRU, MA, and RGPu are shown in Fig. 3. The uranium based fuels display the same general profile, marked by the large peak in the thermal region. The TRUs-, RGPu-, and MAs-bearing fuels produce flux profiles, marked by a peak in the fast energy region and by the absence of a well-pronounced thermal peak.

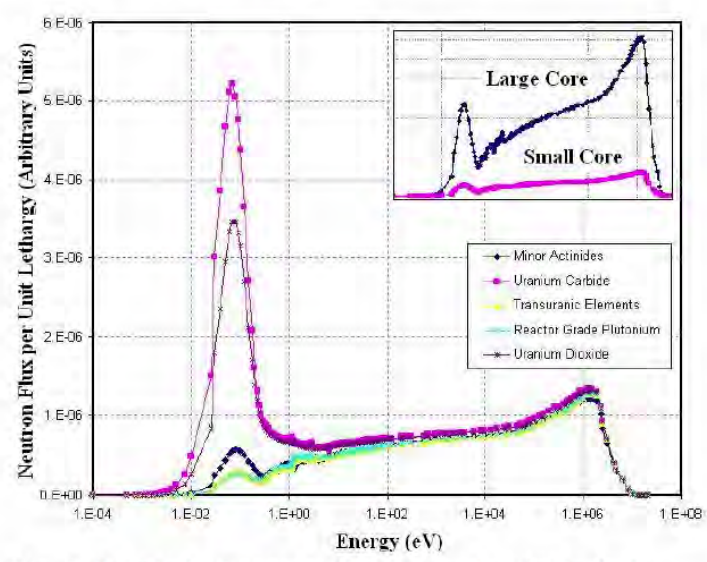

Fig. 3. Pebble-bed cores with advanced actinide fuels

\section{CONCLUSIONS}

The research effort enhances capabilities of the Generation IV VHTR and transforms it to a technology that can deliver electricity, hydrogen, and assist in spent fuel management. Although to be further refined, studies of pebble bed cores with MAs indicate promising performance characteristics.

\section{ACKNOWLEDGMENTS}

This paper is based upon work supported by the U.S. Department of Energy under Award Number DE-FC0705ID14655 (05-094).

\section{REFERENCES}

1. "Evaluation of High Temperature Gas-cooled Reactor Performance: Benchmark Analysis Related to Initial Testing of the HTTR and HTR-10", IAEATECDOC-1382, Austria (2003).

2. C. RODRIGUEZ, A. BAXTER, D. MCEACHERN, M. FIKANI, F. VENNERI, "Deep-Burn: Making Nuclear Waste Transmutation Practical", Nucl. Eng. Design, 2805, 1 (2003).

3. "SCALE: A Modular Code System for Performing Standardized Computer Analyses for Licensing Evaluation", ORNL/TM-2005/39, Ver. 5, April 2005, Oak Ridge National Laboratory, Tennessee (2005).

4. M. PRITCHARD, P. TSVETKOV, "Pebble-Bed Core Design Option for VHTRs - Core Configuration Flexibility and Potential Applications", ANS Topical Meeting on Reactor Phys., Sept. 10-14, 2006, Vancouver, Canada (2006). 
Utilization of MAs as a Fuel Component for Ultra-Long Life VHTR Configurations: Designs, Advantages and Limitations

\title{
6.1 Utilization of TRUs as a Fuel for VHTRs: Compositions, Neutronics Impact and Safety
}

\author{
Pavel V Tsvetkoy, David E. Ames II. Megan L. Priteliard
}

Department of Niclear Enguneering. Texas AdbM Liniversit, 129 Zachry Engineerng Center, MS 3133 TAMU Colloge Station. TX, 77843-3133, E-mail: Isvethovia tomil ediu

\section{INTRODUCTION}

The U.S. Deparment of Energy Advanced Fue Cycle $(A F C)$ program and related international efforts are focused on findug vable nuclear waste management technologies leading to mineration of transurane (TRU) nuchdes. [1] These tecluologes could also serve as an additional fuel supply source.

Due to then promismg fuel cycle adaptability and spectral claaracteristics. High Temperature Gis-cooled Reactors (HTGRs) have always been considered as potential PU/TRU-incinerators. For example, one of the most interesting recent efforts, the Deep-Bum concept, is specifically looking into thermal neutron transmutation of TRU's in the HTGR prismatic cores. [2]

The ongoing U,S. DOE NERI project targets TRU's as a thel for the Generation IV HTGR - Very High Temperature Reactor (VHTR). The use of self-generating compositions based on TRU's should lead to an extended operation without intermediate refueling up to structural limits. The project objective is to determine a design envelop of VHTRs with TRU's as well as their performance advantages (if any). The resultimg core configmations are expected to approach the complete recovery of the energy value of TRUs. This paper discusses the research efforts.

\section{ANALYSIS APPROACH}

A hybrid Monte Carlo-deterministic methodology has been implemented for coupled desigu studies of VHTRs with TRUs. Within the conputational framework, the key technical issues are being addressed and resolved by adopting atutomated modeling procedues and sequences combining Monte Carlo and deternimistic approaclies. developing and applying realistic 3D coupled neutronicsthermal-hydraulics models with multi-heterogeneity treatureuts. Most of the studies is conducted using the ORNL SCALE code system and some auxiliary tools for design sensitivity/uncertainty evaluations. [3]

To assure compreliensive, realistic assessment of the VHTR design and operation largeting passive sifety confinmation, the adequacy of computational methods and models used to compute performance characteristics is supported by comparisons with experimental data covermg an approptiate tange of conditions. The VHTR model benchmark sets were compiled based on the LEUHTR PROTEUS, HTTR, and HTR-10 experimental programs. Validation and verification studies of the applied models and procedures represent the ongowg component of the project work packinge. Up-to-date results are in agreement with the avarlable data and confirm the chosen modeling approach. $[4,5]$

\section{RESEARCH SCOPE}

The project analyzes key aspects of the developed VHTRs with TRUs:

- Reliability aualysis with respect to uncertanity effects due to muclear data and design parameters.

- Configuration variation analysis to demonstrate that the extended-life VHTR with TRU's are feasible.

- Performance analysis to demonstrate that the developed configurations have acceptable control. dynamics, safety and non-proliferation characteristics

Small scale and large scale VHTR units with pebble-bed and prismatic block cores are considered. For example. Fig. 1 shows the prismatic block core layouts.

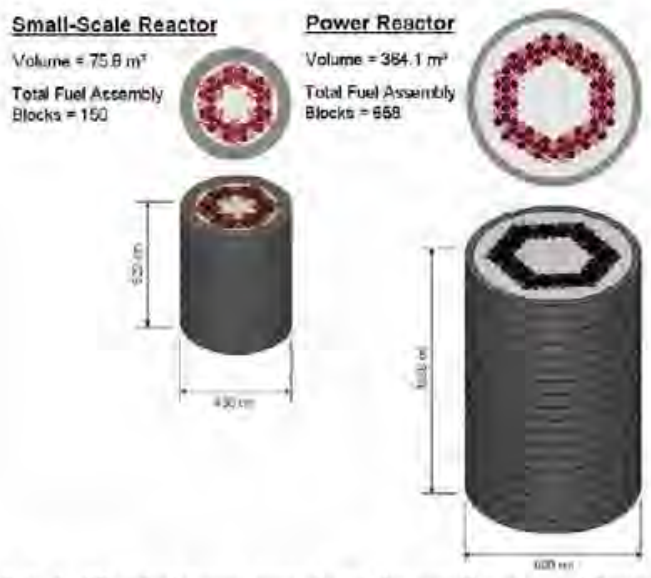

Fig. 1. 3D whole-core models of the small scale and laree scale prismatic block cores (KENO3D plot).

The developed VHTR configurations are analyzed and compared taking into account the existing systems and the concepts with estimated characteristics. The 


\section{Utilization of MAs as a Fuel Component for Ultra-Long Life VHTR Configurations: Designs, Advantages and Limitations}

\section{Project 05-094}

Final Scientific/Technical Report

developed configuration is considered acceptable if it offers similar or better performance.

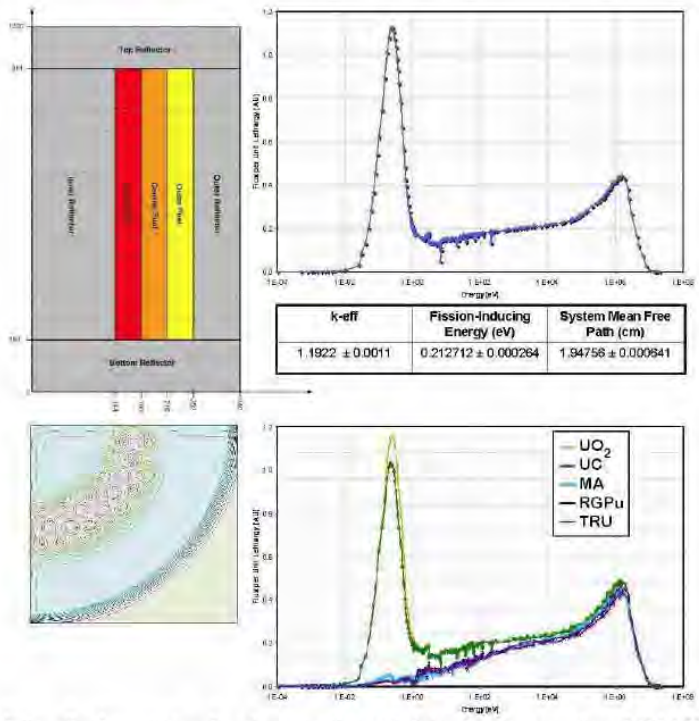

Fig. 2. Impact of advanced actinide fuels on the VHTR energy spectrum (prismatic core layouts and data).

The present study is focused on the VHTR configurations due to their versatility with respect to fuel cycles. It is possible to choose between several fuel forms including advanced actinide fuels. For illustration purposes, Fig. 2 shows the observed spectral variations due to fuel vectors in the prismatic block core. Figure 3 provides the corresponding isothermal core reactivity coefficients.

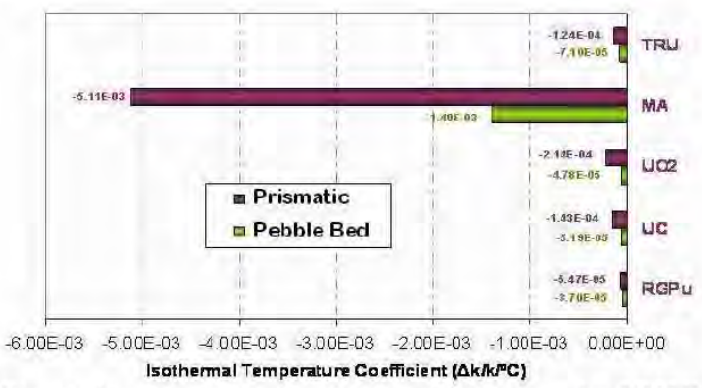

Fig. 3. Impact of advanced actinide fuels on the VHTR safety characteristics (prismatic core layouts and data).

\section{CONCLUSIONS}

The project investigates VHTRs with advanced actinide fuels, which could reduce the long-term radiotoxicity and heat load of high-level waste sent to a geologic repository and enable recovery of the energy contained in spent fuel. The extended-lifetime approach should reduce the technical need for additional repositories and improve marketability of the Generation IV VHTR design. Utilization of TRUs inherently facilitates development of new fuel cycles and supports fuel supply sustainability.

The developed configurations should have inherent capabilities for utilization of TRUs from spent LWR fuel, reduction of spent fuel flows and handling per unit of produced energy, and potential for autonomous operation with minimized maintenance. It is possible to improve fissile properties by neutron spectrum shifting through configuration adjustments. As a result, under specific spectral conditions certain actinides would be able to contribute to the core neutron balance compensating for fuel depletion effects through their chain transformations. The resulting self-stabilization of advanced actinide fuels should allow prolonged operation on a single fuel loading.

Of paramount importance for the development and deployment of the Generation IV VHTR systems is the predicted capability to achieve a high degree of safety through reliance on passive safety features. Up-to-date results demonstrate the significant difference between $\mathrm{UO}_{2}$ - and TRU-fueled VHTRs.

\section{ACKNOWLEDGMENTS}

This paper is based upon work supported by the U.S. Department of Energy under Award Number DE-FC0705ID14655 (05-094).

\section{REFERENCES}

1. "Report to Congress on Advanced Fuel Cycle Initiative: The Future Path for Advanced Spent Fuel Treatment and Transmutation Research", 03GA50439-06, January, 2003, Office of Nuclear Energy, Science, and Technology, U.S. DOE (2003).

2. C. RODRIGUEZ, A. BAXTER, D. MCEACHERN, M. FIKANI, F. VENNERI, "Deep-Burn: Making Nuclear Waste Transmutation Practical", Nucl. Eng. Design, 2805, 1 (2003).

3. "SCALE: A Modular Code System for Performing Standardized Computer Analyses for Licensing Evaluation", ORNL/TM-2005/39, Ver. 5, April 2005, Oak Ridge National Laboratory, Tennessee (2005).

4. "Evaluation of High Temperature Gas-cooled Reactor Performance: Benchmark Analysis Related to Initial Testing of the HTTR and HTR-10", IAEATECDOC-1382, Austria (2003).

5. P. TSVETKOV, et al, "Coupled Hybrid Monte Carlo - Deterministic Analysis of VHTR Configurations with Advanced Actinide Fuels", Proc. Intern. Congress Advances in Nucl. Power Plants (ICAPP 06), Reno, Nevada, June $4-8,2006$, ANS, pp. 1984 1990 (2006).

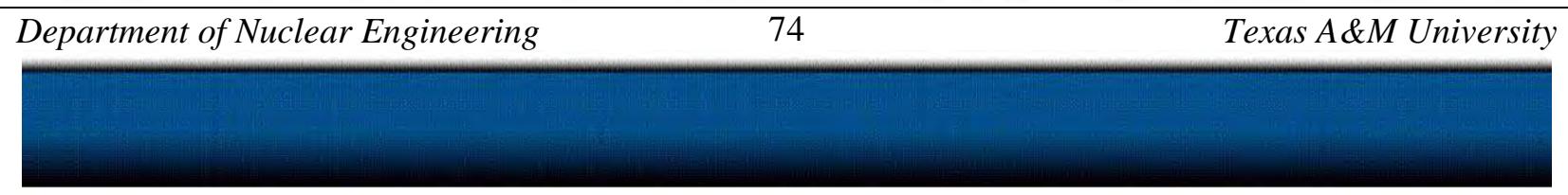


Utilization of MAs as a Fuel Component for Ultra-Long Life VHTR Configurations: Designs, Advantages and Limitations

Project 05-094

Final Scientific/Technical Report

\title{
6.2. Analysis of TRU-Fueled VHTR Prismatic Core Performance Domains
}

\author{
*Tom G. Lewis III. **David E. Ames II, ***"Pavel V. Tsvetkov
}

Department of Nuclear Engineering. Texas A\&M University, 129 Zachry Engineering Center, MS 3133 TAMU College Station, TX,77843-3133,E-mails: "tglewis3@gmail.oom. "deames 99@yahoo.com, "*ktsvetkov@atamu.edi

\section{INTRODUCTION}

The nuclear waste management technologies, which involve TRU vectors as a fuel, not only provide an additional fuel supply but also offer the ability to extend operation without intermediate refueling up to structural limits. [1.2] Due to favorable perfonmance characteristics, multiple studies also focus on transmutation options using High Temperature Gas-cooled Reactors (HTGRs). [2,3]

The objective of the present 3-year U.S. DOE NERI project is to determine a design envelop of TRU-fueled VHTRs operating in an extended batch mode without refueling. The resulting TRU-core configurations are expected to be suitable for long- term autonomous operation. This paper discusses research efforts within the project.

\section{MODEL IMPLEMENTATION}

A Monte Carlo-deterministic analysis methodology has been implemented for coupled design studies of VHTRs with TRUs using the ORNL SCALE 5.1 code system. [4] Figure I shows applied 3D whole-core model structure and $3 \mathrm{D}$ temperature distribution that correspond to prototypic operation conditions [5] The modeling adequacy is confirmed by performing series of experiment-to-code benchmark evaluations. [6]

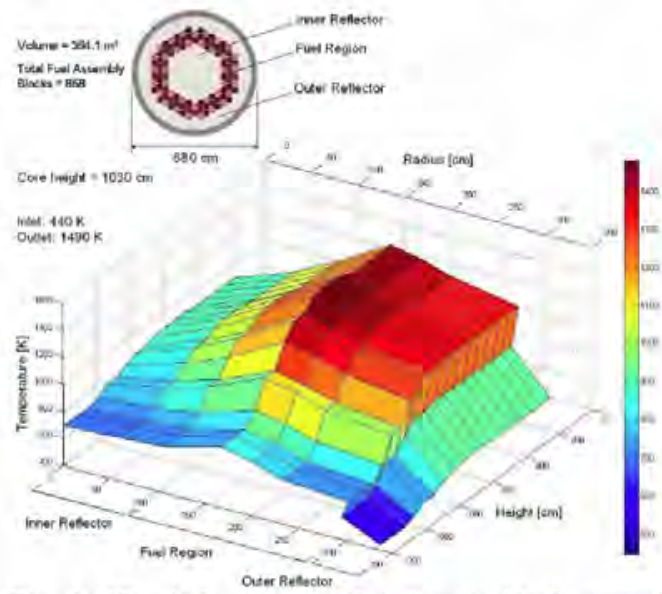

Fig. 1. 3D whole-core model (prismatic block system) including a representative temperature distribution.

\section{RESULTS}

Up-to-date studies demonstrate significant differences between the LEU- and TRU-fueled VHTR configurations. Figure 2 shows neutron spectra in the VHTR prismatic cores for several carbon-to-heavy metal (C/HM) atom ratios. In this analysis, the $\mathrm{C} / \mathrm{HM}$-variations are archived by considering different numbers of TRISO particles per compact.
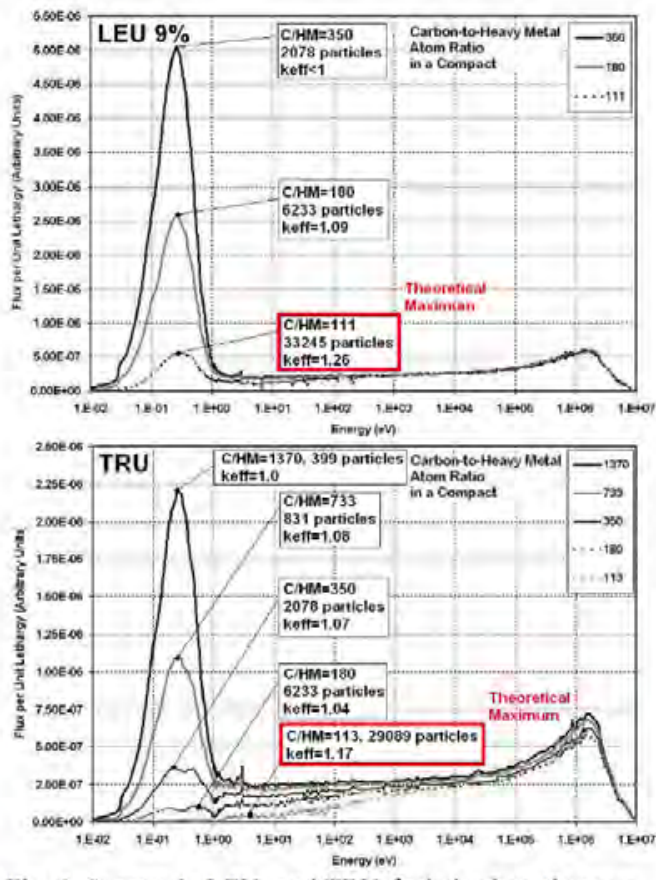

Fig. 2. Spectra in LEU- and TRU-fueled prismatic cores.

In both cases, LEU and TRU, variations of numbers of particles per compact result in complex changes of the VHTR core performance characteristics. In particular, the thermal neutron population decreases in the TRU-fueled VHTR cores as a function of the C/HM atom ratio. For comparable low $\mathrm{C} / \mathrm{HM}$ atom ratio values, there is almost no peaking of the flux at thermal energies in the TRUfueled cores whereas the LEU-fueled cores still exhibit distinctive thermal spectra. This behavior is to be expected, accounting for nuclear physics of TRU vectors 


\section{Utilization of MAs as a Fuel Component for Ultra-Long Life} VHTR Configurations: Designs, Advantages and Limitations

\section{Project 05-094}

\section{Final Scientific/Technical Report}

and reduced moderation in the VHTR cores at low $\mathrm{C} / \mathrm{HM}$ atom ratio values.

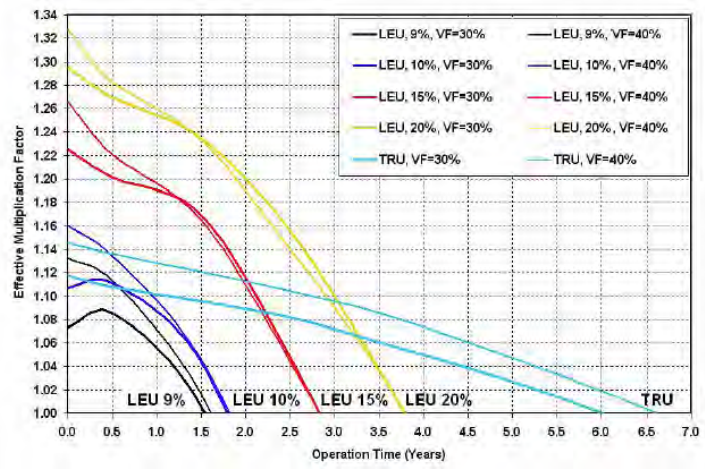

Fig. 3. Batch mode operation potential of VHTRs.

As illustrated in Fig. 3, the TRU-fueled VHTR cores can potentially sustain longer refueling intervals than the corresponding LEU-fueled traditional VHTR cores. The extended operation of the TRU-fueled VHTRs is also characterized by smaller lifetime reactivity swings due to self-sufficiency of advanced actinide compositions. The extended batch mode operation without refueling can be prolonged further by decreasing power densities in the TRU-fueled VHTR cores to reduce fast fluences. Figure 4 demonstrates limiting fast fluences that will force power density reductions and use of advanced materials. These limitations will ultimately define performance domains of VHTRs with advanced actinide fuels.

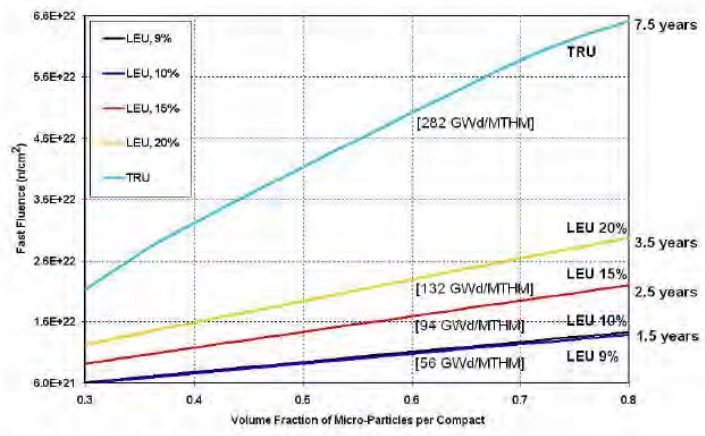

[..]-Max. achievable bumup levels in considered core configurations

Fig. 4. Performance domains of VHTRs (LEU and TRU).

\section{CONCLUSIONS}

The extended-lifetime approach could reduce the technical need for additional repositories and should also improve marketability of the Generation IV VHTR designs as small-to-medium internationally deployable energy sources for electricity generation and industrial heat applications. The TRU-fueled VHTRs offer performance characteristics that would be difficult to achieve in analogous LEU-fueled systems:

- Almost a decade-long batch mode operation without intermediate refueling:

- Significant reductions of initial excess reactivity levels (smaller lifetime reactivity swings);

- Inherently higher achievable burnup levels.

At the same time, the presented results clearly illustrate that use of TRU vectors as a fuel inherently facilitates development of specially designed VHTRs with core materials withstanding performance conditions of systems optimized for fuel loading with TRUs:

- Limiting fast fluences and larger resulting radiation damage effects:

- Significant core physics differences due to spectral shift effects towards harder neutron spectra with substantially reduced neutron populations at thermal energies.

\section{ACKNOWLEDGMENTS}

This paper is based upon work supported by the U.S. Department of Energy under Award Number DE-FC0705ID14655 (05-094).

\section{REFERENCES}

1. "Report to Congress on Advanced Fuel Cycle Initiative: The Future Path for Advanced Spent Fuel Treatment and Transmutation Research", 03GA50439-06, U.S. DOE (2003).

2. P. TSVETKOV, A. ALAJO, D. AMES, M. PRITCHARD, "Spectrum Shifting as a Mechanism to Improve Performance of VHTRs with Advanced Actinide Fuels", Proc. 14th Intern. Conf. Nucl. Eng. (ICONE 14), Miami, Florida, July 17 - 20, 2006. ASME (2006).

3. T. KIM, T. TAIWO, R. HILL, W. YANG, F. VENNERI, "A Feasibility Study of Reactor-Based Deep-Burn Concepts", ANL-AFCI-155, Argonne National Laboratory (2005).

4. "SCALE: A Modular Code System for Performing Standardized Computer Analyses for Licensing Evaluation", ORNL/TM-2005/39,Ver.5.1, November 3, 2006, Oak Ridge National Laboratory, Tennessee (2006).

5. H. HAQUE, W. FELTES, G. BRINKMANN, "Thermal Response of a Modular High Temperature Reactor during Passive Cooldown under Pressurized and Depressurized Conditions", Nucl. Eng. Design, 236, 475 (2006).

6. "Evaluation of High Temperature Gas-cooled Reactor Performance: Benchmark Analysis Related to Initial Testing of the HTTR and HTR-10", IAEATECDOC-1382, Austria (2003). 


\subsection{TRU-Fueled VHTRs: Design, Performance and Applications}

Pavel V. Tsvetkov, David E. Ames II, Megan L. Pritchard, Ayodeji B. Alajo, and Tom G. Lewis III

Nuclear Engineering Dept., Texas A\&M University, 129 Zachry, MS 3133, College Station, TX, 77843, tsvetkov@.tamu.edu

The Very High Temperature Reactor (VHTR) is the nearest Generation IV concept. This paper presents results of the U.S. DOE NERI Project on utilization of higher actinides (TRUs and partitioned MAs) as a fuel component for extended-life VHTR configurations. The idea is to approach autonomous operation on a single fuel loading that would allow marketing power units with VHTRs as nuclear batteries for worldwide deployment. The research focus is on possible designs and their advantages and limitations. The project is currently within its third year of studies. Although indicating some technical limitations and challenges, studies of VHTRs with TRUs/MAs definitely suggest promising performance and possibility to utilize the core configurations with TRUs/MAs gaining prolonged operation and selfsustainability.

\section{INTRODUCTION}

The Very High Temperature Reactor (VHTR) is the nearest Generation IV concept. It is designed to be a highefficiency system, which can supply electricity and process heat to a range of high temperature and energyintensive applications.

The basic technology has been well established in High Temperature Gas Reactors (HTGR), such as the German AVR and THTR prototypes, and the U.S. Fort Saint Vrain and Peach Bottom prototypes. The VHTR extends the capabilities of HTGRs to achieve further improvements in thermal efficiency and establish a technological basis for emerging high-temperature applications.

This paper presents the results of the U.S. DOE NERI Project on utilization of higher actinides (TRUs and partitioned MAs) as a fuel component for extended-life VHTR configurations. Both, pebble-bed and prismatic block core concepts are considered.

The idea is to approach autonomous operation on a single fuel loading that would allow marketing power units with VHTRs as nuclear batteries for worldwide deployment. The research focus is on possible designs and their advantages and limitations.

\section{VHTR SYSTEMS WITH TRU LOADINGS}

Utilization of TRUs/MAs in VHTRs facilitates development of advanced fuel cycles and supports fuel supply sustainability. ${ }^{1}$ Under certain spectral conditions, TRUs/MAs would be able to contribute to a core neutron balance compensating for depletion. ${ }^{2}$ The resulting selfstabilization of advanced actinide fuels is expected to prolong operation on a single fuel loading up to lifetimes limited by structural/integrity characteristics.

A TRUs/MAs-bearing fuel is the major challenge in the development of the partitioning and transmutation engineering technologies. To support analysis of the VHTR configurations with advanced actinide fuels, the studies were focused on actinide compounds that are currently being considered and/or are under development for use as TRUs/MAs-bearing transmutation fuels. ${ }^{3}$ This information is used to facilitate realistic studies of the VHTRs with MAs.

Variability of advanced fuel forms and their characteristics as well as variations of TRU vectors are taken into account as uncertainties. For example, the relative sensitivity of TRU vectors to burnup is shown in Fig. 1.

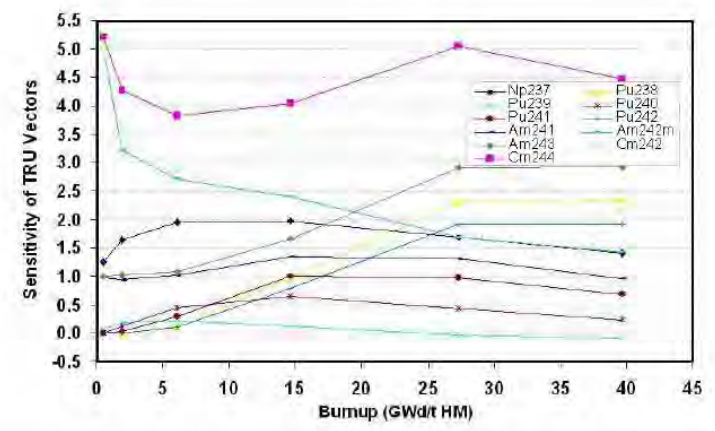

Fig. 1. Sensitivity of TRU vectors to reactor operation conditions (typical PWR conditions, 1000MW (e)).

The actual PWR spent fuel data allow eliminating purely theoretical configurations. ${ }^{4}$ Figure 2 illustrates 


\section{Utilization of MAs as a Fuel Component for Ultra-Long Life} VHTR Configurations: Designs, Advantages and Limitations

\section{Project 05-094}

Final Scientific/Technical Report

variations of MA vectors as a function of increasing PWR spent fuel burnup.

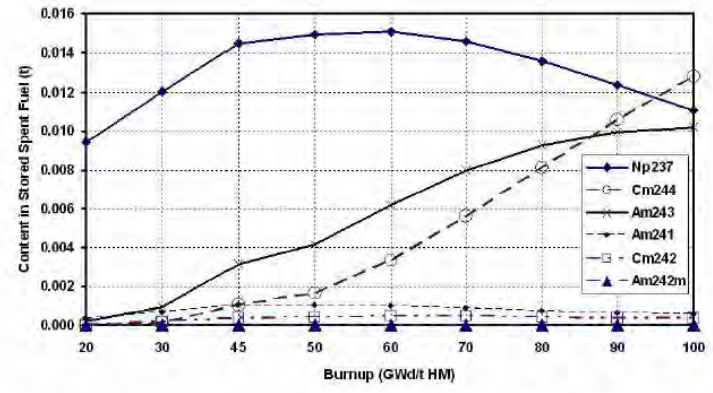

Fig. 2. Variations of MA-vectors in the stored spent fuel (typical PWR conditions, $1000 \mathrm{MW}(\mathrm{e})$ ).

\section{SYSTEM MODELING AND V\&V EFFORTS}

A hybrid Monte Carlo-deterministic methodology has been adopted for coupled neutronics/thermal hydraulics/fuel cycle design studies of VHTRs. Within the computational scheme, the key technical issues are being addressed and resolved by implementing efficient automated modeling procedures and sequences, combining Monte Carlo and deterministic approaches, developing and applying realistic 3D coupled neutronicsthermal-hydraulics models with multi-heterogeneity treatments, developing and performing experimental/computational benchmarks for model verification and validation, analyzing uncertainty effects and error propagation The results describe performance of the entire system, including a power unit and its associated fuel cycle, and allow conclusions regarding the configuration's feasibility, performance, and possible directions for further development.

To assure comprehensive and realistic assessment, the VHTR model benchmark sets were compiled based on the LEU-HTR PROTEUS, HTTR, and HTR-10 experimental programs. ${ }^{5}$ Validation and verification of the VHTR models and the analysis of uncertainty effects on VHTR performance characteristics including the preliminary studies of the VHTRs with MAs are completed. Up-to-date results are in agreement with the available data and confirm the chosen approach. The developed formalism provides the basis for the chosen uncertainty analysis methodology and for the selection of appropriate tools.

The code systems and tools for evaluations of uncertainty effects (nuclear and design/performance uncertainties) were developed on the basis of existing software packages to support developed uncertainty analysis methodologies. The chosen approach links the applied suite of neutronics and thermal hydraulics codes and the generalized codes for universal sensitivity analysis, calibration and uncertainty evaluations in a framework.

The project tools include MCNP, SCALE, auxiliary tools to account for double heterogeneity, HeatWave suite, and statistical analysis packages and modules. ${ }^{6}$ These tools form the code system for coupled modeling of VHTRs. The generalized codes for universal sensitivity analysis, calibration and uncertainty evaluations are represented by UCODE-2005 code system (by U.S. Geological Survey) and its post-processors.

\section{ANALYSIS OF TRU-FUELED VHTRS}

The work is a comprehensive analysis based on realistic design-level system modeling. The analysis includes all aspects of model development, validation and verification based on experimental data, system design development and performance assessment.

Based on the initial studies of the VHTR with TRUs/MAs, the major thrust is the detailed analysis of configuration variation capabilities to achieve prolonged operation without refueling, maximize burnup, and minimize reactivity swings. Using $3 \mathrm{D}$ whole-core/system models, researchers developed a variation analysis methodology and then applied it to studies of VHTR geometry and material variations. Advantages and limitations of an extended single-batch operation are illustrated in Fig. 3. Figure 4 shows evolving safety characteristics as a function of operation time (core lifetime in these studies).

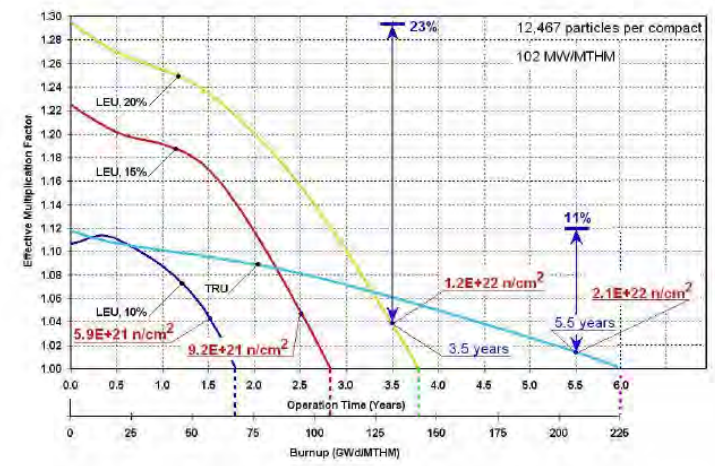

Fig. 3. Performance characteristics of LEU- and TRUfueled VHTRs (hexagonal block configurations operating in a single-batch mode).

Operation of the TRU-fueled VHTRs is characterized by larger burnup values and potentially smaller reactivity swings during a single batch-mode operation. The core lifetime is clearly limited by fast neutron fluences. This constraint can be relaxed or even eliminated by reducing power density levels and using advanced radiation- 


\section{Utilization of MAs as a Fuel Component for Ultra-Long Life} VHTR Configurations: Designs, Advantages and Limitations

\section{Project 05-094}

\section{Final Scientific/Technical Report}

tolerant materials for extended-life VHTR configurations Safety characteristics of the TRU-fueled VHTRs retain their acceptable values during considered core lifetimes.

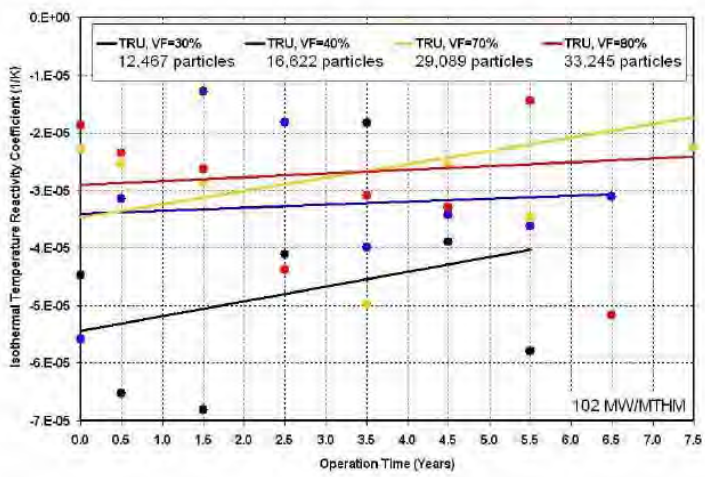

Fig. 4. Trends in the TRU-fueled VHTR safety characteristics during a single batch-mode operation.

Figure 5 shows spectral transitions during operation in the considered LEU- and TRU-fueled VHTRs. The SCALE-computed typical PWR spectrum is shown for comparison.

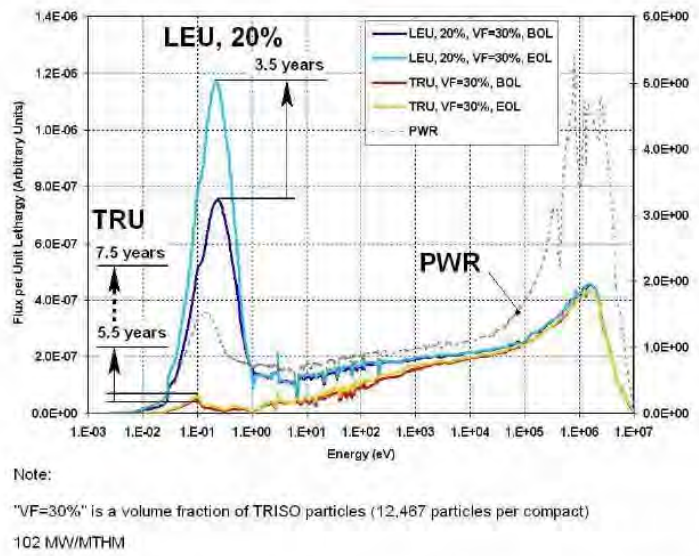

Fig. 5. Neutron spectra in LEU- and TRU-fueled VHTRs (hexagonal block configurations) and transitions during a single batch-mode operation.

As illustrated in Fig. 5, there are characteristic spectral transitions in the LEU-fueled extended-life VHTR configurations ( 3.5 years on a single fuel loading without intermediate shuffling or refueling). The TRUfueled VHTR configurations exhibit very specific neutron spectra characterized by very small thermal peaks and minimal transitions during operation $(5.5$ years on a single fuel loading without intermediate shuffling or refueling).

The analysis of existing uncertainty effects on VHTR performance characteristics is focused on effects of nuclear data and design parameters. The uncertainty analysis is followed by the reliability evaluation of the VHTR modeling. It yields evaluations of an overall modeling reliability with identification of a computational design envelop expected for the VHTR configurations with TRUs/MAs. When further generalized, this methodology will be suitable for multi-parametric design optimization.

The performed sensitivity/uncertainty analysis is the basis for the ongoing studies of configuration variation capabilities. The goal is to evaluate and optimize a physically achievable system lifetime and the existing prolongation potential taking advantage of selfsustainable fuel compositions with higher actinides. The design target is an autonomous operation with minimized required control interventions.

Depending on dominant neutron energies in the considered core configurations, TRUs/MAs may have high neutron yields or act as absorbers. The observations are not completely conclusive because of uncertainties in nuclear data.

Besides data uncertainties, the enhanced involvement of TRUs/MAs is further complicated by their low delayed neutron yields. Significant variations of MA delayed neutron yields per fission neutron released are shown in Fig. 6. Delayed neutron yields for some of MAs are several orders of magnitude lower than for ${ }^{238} \mathrm{U}$. These variations suggest design challenges because of dynamics and safety characteristics of the VHTRs with advanced actinide fuels.

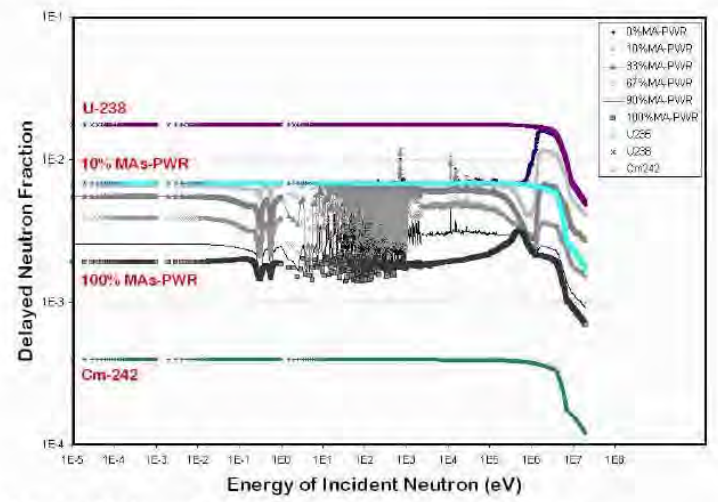

Fig. 6. Delayed neuron fractions of MA-mixtures.

\section{CONCLUSIONS}

The project is currently within its third year of studies. Although indicating some technical limitations 


\section{Utilization of MAs as a Fuel Component for Ultra-Long Life VHTR Configurations: Designs, Advantages and Limitations}

and challenges, studies of VHTRs with TRUs/MAs definitely suggest promising performance and possibility to utilize the core configurations with TRUs/MAs gaining prolonged operation and self-sustainability:

- There are significant differences between LEUand TRUs-fueled VHTRs. These differences affect system performance characteristics during operation.

- A single batch-mode operation appears to be potentially possible. Core lifetimes up to 7.5 years have been observed in the present analysis.

- Fluence-related limitations (radiation damage) are the most significant constraints on achievable operation times. This constraint can be relaxed or even eliminated by reducing power density levels and using advanced radiation-tolerant materials for extended-life VHTR configurations. However, use of advanced materials will adversely impact economics characteristics.

- Acceptable safety characteristics have been observed for all configurations. However, low delayed neutron yields may result in design challenges.

The discussed, currently ongoing studies are the central, the most time consuming and information-intense. part of the project.

\section{ACKNOWLEDGMENTS}

This paper is based upon work supported by the U.S. Department of Energy under Award Number DE-FC0705ID14655 (05-094).

\section{REFERENCES}

1. "Report to Congress on Advanced Fuel Cycle Initiative: The Future Path for Advanced Spent Fuel Treatment and Transmutation Research", 03GA50439-06, January, 2003, Office of Nuclear Energy, Science, and Technology, U.S. DOE (2003).

2. P. TSVETKOV, A. ALAJO, D. AMES, M. PRITCHARD, "Spectrum Shifting as a Mechanism to Improve Performance of VHTRs with Advanced Actinide Fuels", Proc, 14th Intern. Conf. Nucl. Eng. (ICONE 14), Miami, Florida, July 17 - 20, 2006, ASME (2006).

3. T. KIM, T. TAIWO, R. HILL, W. YANG, F. VENNERI, "A Feasibility Study of Reactor-Based Deep-Burn Concepts", ANL-AFCI-155, Argonne National Laboratory (2005).

4. "Final Environmental Impact Statement for a Geologic Repository for the Disposal of Spent Nuclear Fuel and High-Level Radioactive Waste at Yucca Mountain, Nye County, Nevada", Vol. 2, Appendix A, "Inventory and Characteristics of Spent
Nuclear Fuel, High-Level Radioactive Waste, and Other Materials", DOE/EIS-0250, U.S. DOE (2002).

5. "Evaluation of High Temperature Gas-cooled Reactor Performance: Benchmark Analysis Related to Initial Testing of the HTTR and HTR-10", IAEATECDOC-1382, International Atomic Energy Agency, Vienna, Austria (2003).

6. "SCALE: A Modular Code System for Performing Standardized Computer Analyses for Licensing Evaluation", ORNL/TM-2005/39, Ver. 5.1, November 2006, Oak Ridge National Laboratory, Oak Ridge, Tennessee (2006). 


\title{
6.4. TRU-Fueled VHTRs for Applications Requiring an Extended Operation with Minimized Control and No Refueling
}

\author{
Pavel V. Tsvetkov, Tom G. Lewis III, Ayodeji B. Alajo \\ Department of Nuclear Engineering \\ Texas A\&M University \\ 129 Zachry Engineering Center, MS 3133 TAMU, College Station, Texas 77843-3133, USA \\ Phone: 979/845-7078, \\ Fax: 979/845-6443 \\ E-mail: Tsvetkov@tamu.edu
}

\section{KEYWORDS}

Transuranics, VHTR, refueling, extended operation

\begin{abstract}
This paper presents an analysis of TRU-fueled VHTR systems focusing on applications requiring an extended operation with minimized control and no refueling (singlebatch mode). As an example of such applications, international deployment opportunities for grid-appropriate VHTR systems could be mentioned addressing demands for electricity, industrial heat and co-generation in those regions where minimized servicing is desirable for various reasons. The study is performed for the hexagonal block core concept within the framework of the ongoing U.S. DOE NERI Project on utilization of higher actinides (TRUs and partitioned MAs) as a fuel component for extended-life VHTRs. The up-to-date analysis has shown reasonable reactivity swings, core life limits with respect to fast fluences and criticality.
\end{abstract}

$\begin{array}{cl}\text { NOMENCLATURE } \\ \text { DU } & \text { - Depleted Uranium } \\ \text { FP } & \text { - Fission Products } \\ \text { HTGR } & \text { - } \text { High Temperature Gas-cooled Reactor } \\ \text { LEU } & \text { - Low-Enriched Uranium } \\ \text { NERI } & \text { - Nuclear Energy Research Initiative } \\ \text { PWR } & \text { - Pressurized Water Reactor } \\ \text { SNF } & \text { - Spent Nuclear Fuel } \\ \text { TF } & \text { - Transmutation Fuel } \\ \text { TRU } & \text { - Transuranium Nuclides } \\ \text { VHTR } & - \text { Very High Temperature Reactor }\end{array}$

\section{INTRODUCTION}

New approaches to nuclear waste management are being developed. They involve recovery and reuse of TRUs in thermal and fast reactors to reduce the amount of wastes requiring permanent geological disposal options and to facilitate nuclear energy sustainability. [1] Multiple studies explore TRU-bearing fuels for use in next generation nuclear systems. [2] In this context, it is viable to consider PWR spent fuel vectors as being reprocessed and recycled for further utilization as transmutation fuel feeds of VHTRs. [3] As generation III+ reactors phase out, the TRU-fueled VHTRs would become a dominant thermal reactor transmutation option taking advantage of unique characteristics of TRUs. [4]

The study is performed for the hexagonal block core concept within the framework of the ongoing U.S. DOE NERI Project on utilization of higher actinides (TRUs and partitioned MAs) as a fuel component for extended-life VHTRs. [5] Researchers analyze TRU-fueled VHTR configurations and their capabilities to approach the extended operation with minimized reactivity swing and no refueling. The TRU-fueled systems are compared to the VHTR cases with low enriched uranium (LEU). The up-to-date analysis has shown reasonable reactivity swings, core life limits with respect to fast fluences and criticality.

To assure comprehensive and realistic assessment of the VHTR design parameters and performance characteristics targeting safety confirmation, the adequacy of the applied computational methods and models is justified through validation \& verification (V\&V) comparison series with experimental data covering appropriate ranges of conditions. $[6,7]$ The use of Monte Carlo codes creates a potential for explicit 3D whole-core/reactor studies. [8]

\begin{tabular}{lll} 
Department of Nuclear Engineering & 81 & Texas A\&M University \\
\hline & &
\end{tabular}




\section{Utilization of MAs as a Fuel Component for Ultra-Long Life VHTR Configurations: Designs, Advantages and Limitations}

\section{Project 05-094}

Final Scientific/Technical Report

Depending on neutron spectra, neptunium, americium and curium may contribute to small reactivity swings (selfstabilization) over prolonged irradiation periods. As a result, under certain spectral conditions TRUs would be able to contribute to the VHTR core neutron balance compensating for fuel depletion effects through their chain transformations. Selfstabilization features of advanced actinide fuels should facilitate capabilities of VHTRs to sustain a single-batch mode operation. The resulting configurations should also have an inherent potential for autonomous operation with minimized maintenance. Their implementation will allow worldwide deployment including developing countries and remote areas.

Flexibility of VHTRs with respect to their fuel cycle choices is one of the well-known features of HTGRs. Tolerance to potential variations of fuel loading vectors is especially important for binary systems with significantly varying characteristics of their constituents like PWRs and VHTRs. As envisioned in the present analysis, Fig. 1 shows a symbiotic quasi-sustainable system consisting of PWRs and TRU-fueled
VHTRs that takes advantage of this feature. Because of fissile feeds (like LEU (fresh) in Fig. 1), only quasi-sustainability can be claimed for asymptotic configurations where external LEUfeeds of PWRs approach zero, $\vec{N}_{L E U}^{(0)} \rightarrow 0$, as internal transmutation fuel feeds of VHTRs (while PWRs phase out) move toward equilibrium, $\vec{N}_{I F}^{(0,0)} \rightarrow \ldots \rightarrow \bar{N}_{I F}^{(0, i)} \rightarrow \bar{N}_{I F}^{(0, \infty)}$.

In the present analysis, potential PWR spent fuel vectors. $\vec{N}_{S N F}^{(0)}$, were determined using the LAEA's nuclear fuel cycle simulation system (VISTA code system). To evaluate potential perturbations in $\bar{N}_{S N F}^{(0)}$ and their influence on the TRU-fueled VHTR performance characteristics, fuel burnup and load factor values for PWRs were perturbed. The reference $\vec{N}_{S N F}^{(0)}$ was determined from the analysis of legacy spent fuel vectors of PWRs. [9]

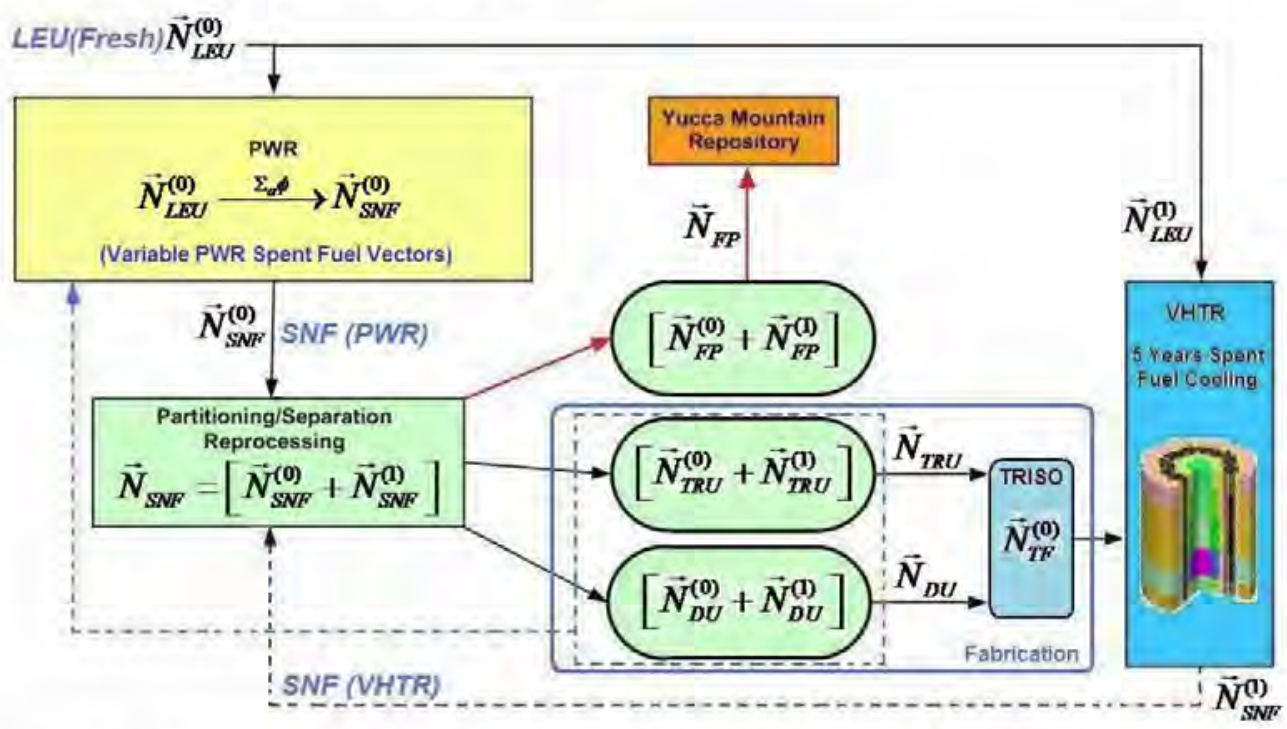

Figure 1. Symbiotic quasi-sustainable PWR-VHTR system.

\section{HIGH FIDELITY MODELING APPROACH}

To create advanced nuclear energy systems it is desirable to have a high fidelity modeling-based design development that relies on simulating features of the entire life cycle of the system before actual physical prototyping - from concept development to detailed design, prototyping, and safety analysis. This approach would provide consistency between neutronics and other performance aspects such as thermal hydraulics, mechanical integrity, plant heat transfer and conversion balances. The suggested integrated/hybrid Monte-
Carlo-deterministic modeling approach offers the desirable analysis capabilities for the VHTR design studies. The use of Monte Carlo codes creates a potential for explicit 3D wholecore/reactor studies of the VHTR configurations addressing the key reactor physics areas such as the double and multi-level heterogeneity, neutron streaming in the low-density regions. and neutron spectrum transitions at the interfaces.

The 3D whole-core exact-geometry VHTR model has been developed for use in calculations with SCALE 5.1 code system. [10] As shown in Fig. 2, the model takes into account TRISO micro-particles, fuel compacts, fuel assembly blocks, control 


\section{Utilization of MAs as a Fuel Component for Ultra-Long Life} VHTR Configurations: Designs, Advantages and Limitations

rod blocks, replaceable reflectors and a permanent outer reflector. The fuel assembly blocks are based on the HTTR fuel block configuration. The SCALE 5.1 double-heterogeneity treatment supports quasi-explicit representation of TRISO micro-particles within a compact fuel region.

The adequacy of the applied computational methods and models used to evaluate performance has been assessed and confirmed by comparisons with experimental data covering appropriate ranges of conditions. Validation data are available from research reactors and zero-power critical assemblies. The project uses the actual test results and benchmark problems of the HTTR Program (prismatic core design, reactor experiments). Observed differences are taken into account at the implementation and interpretation stages of the present analysis. The modeling results are obtained using general material properties and standard SCALE cross section libraries without any special tailoring to specific systems.

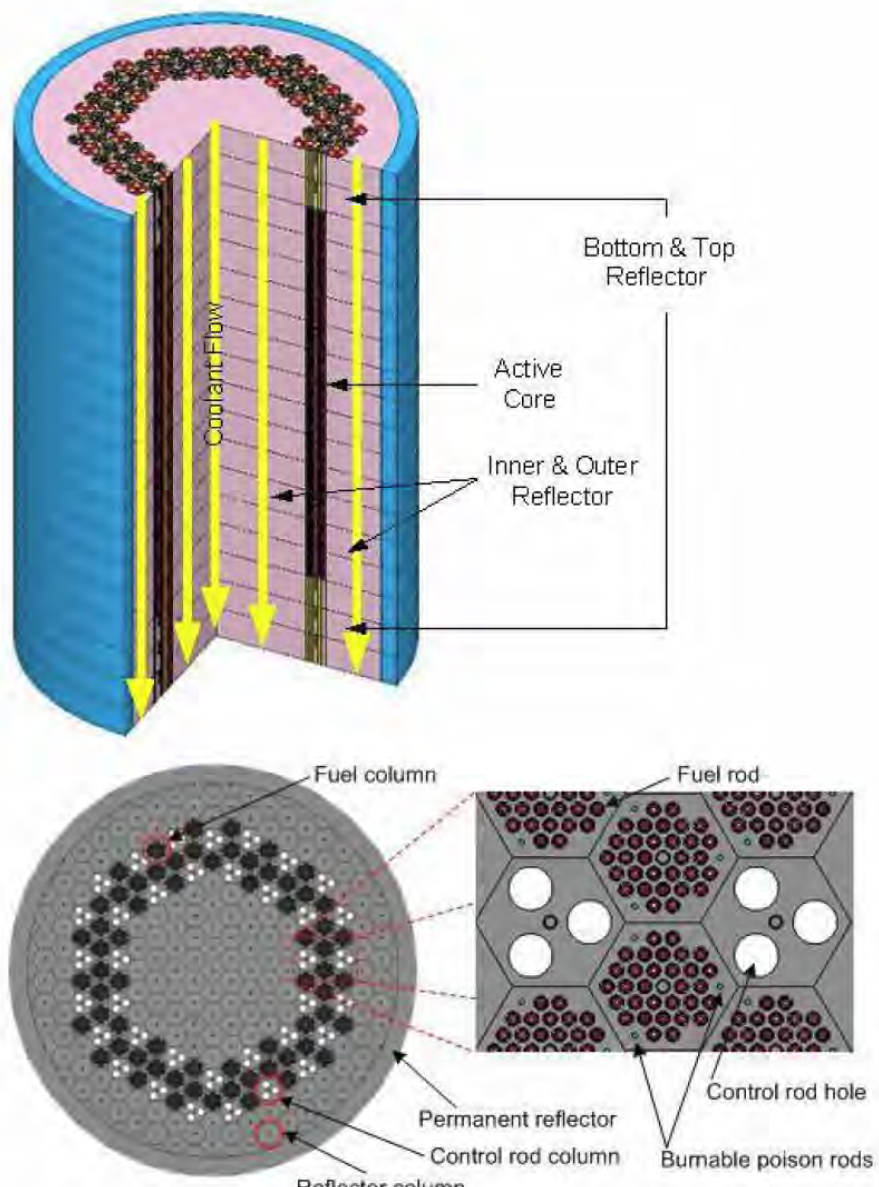

Figure 2. 3D whole-core exact-geometry VHTR model.

\section{NEUTRON SPECTRA IN VHTR CONFIGURATIONS}

Figure 3 illustrates 3D spectral effects in VHTRs due to fuel vector variations. In all cases, there are noticeable neutron leakage effects at the core ends contributing to decreasing thermal and fast flux peak magnitudes. To produce a distinct thermal neutron peak in TRU-fueled VHTR configurations, much larger carbon-to-heavy metal ( $\mathrm{C} / \mathrm{HM})$ ratios should be chosen. For comparable C/HM ratios in the critical LEU- and TRU-fueled configurations, the VHTR system with TRUs does not have a distinct thermal peak. 


\section{STR \\ Research Initiative}

Utilization of MAs as a Fuel Component for Ultra-Long Life

VHTR Configurations: Designs, Advantages and Limitations

Project 05-094

Final Scientific/Technical Report

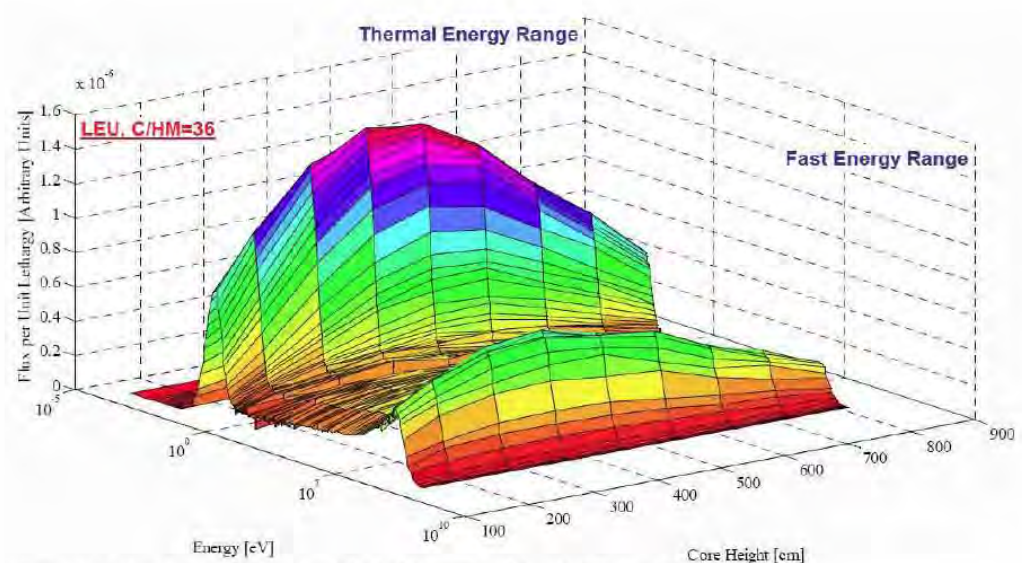

Three fuel-ring LEU $(7.92$ at \%) fueled VHTR with a C/HM atom ratio of 36 in the innermost fuel ring

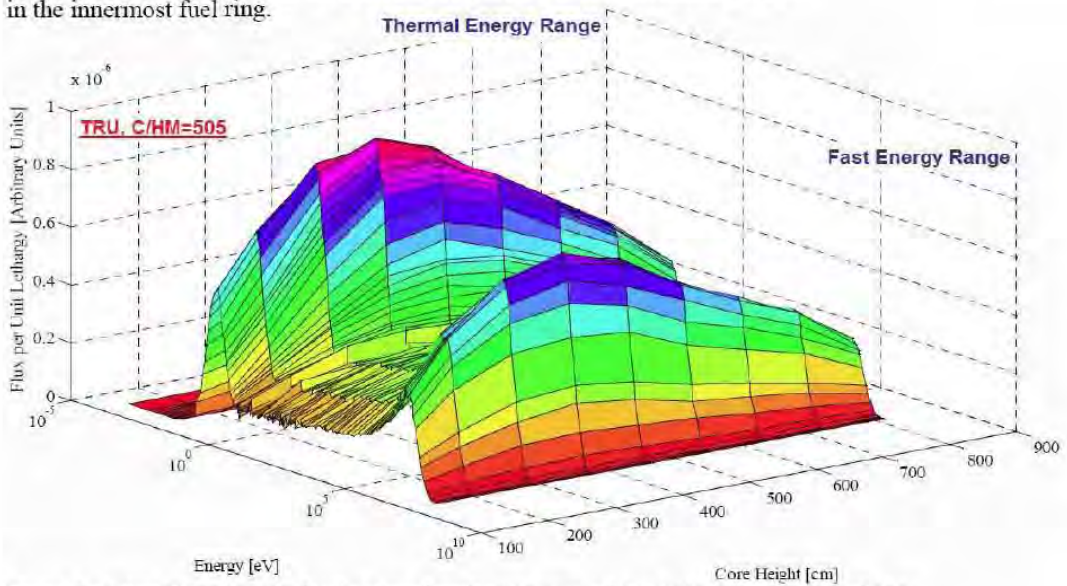

Three fuel-ring TRU (55.86 fissile atom fraction) VHTR with a $\mathrm{C} / \mathrm{HM}$ atom ratio of 505 in the innermost fuel ring.

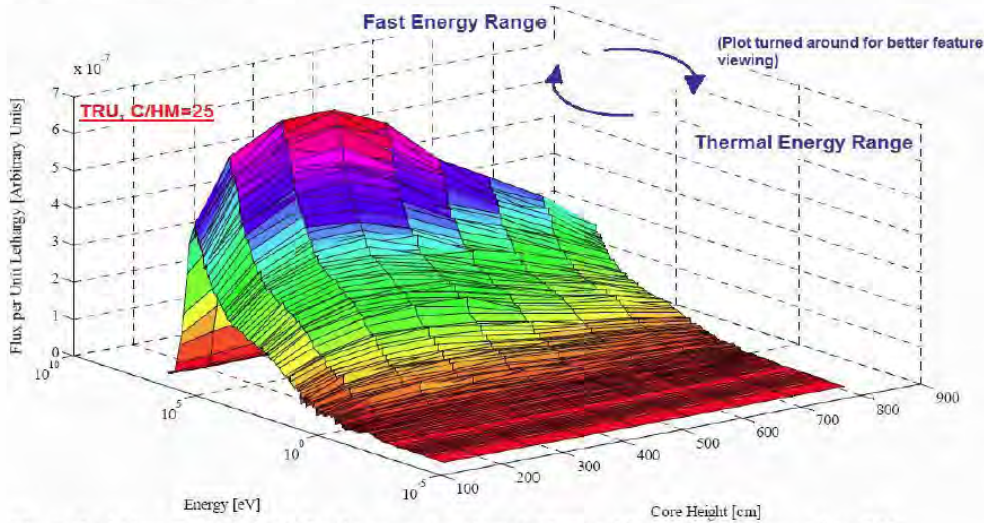

Three fuel-ring TRU (55.86 fissile atom fraction) VHTR with a $\mathrm{C} / \mathrm{HM}$ atom ratio of 25 in the innermost fuel ring.

Figure 3. Fuel effects in VHTRs - spectral variations. 
Utilization of MAs as a Fuel Component for Ultra-Long Life VHTR Configurations: Designs, Advantages and Limitations

VHTR CONFIGURATIONS IN A SINGLE-BATCH MODE

The use of coated micro-particles creates gives flexibility in fuel selection, management. core performance and adaptability. The VHTR multi-heterogeneity features allow material separation by using different types of micro-particles and graphite blocks. The degree of heterogeneity and the moderator-to-ficl ratio ( $\mathrm{C} / \mathrm{HM}$ atom ratio) can be adjusted to achieve desirable spectrum shifting.

Several TRU-fiueled VHTR configurations have been analyzed to determine the effect of the $\mathrm{C} / \mathrm{HM}$ atom ratio on the single-batch core lifetime. Fig. 4 illustrates the TRU-fueled VHTR operation in a single-batch mode as a function of the C/HM atom ratio per compact.

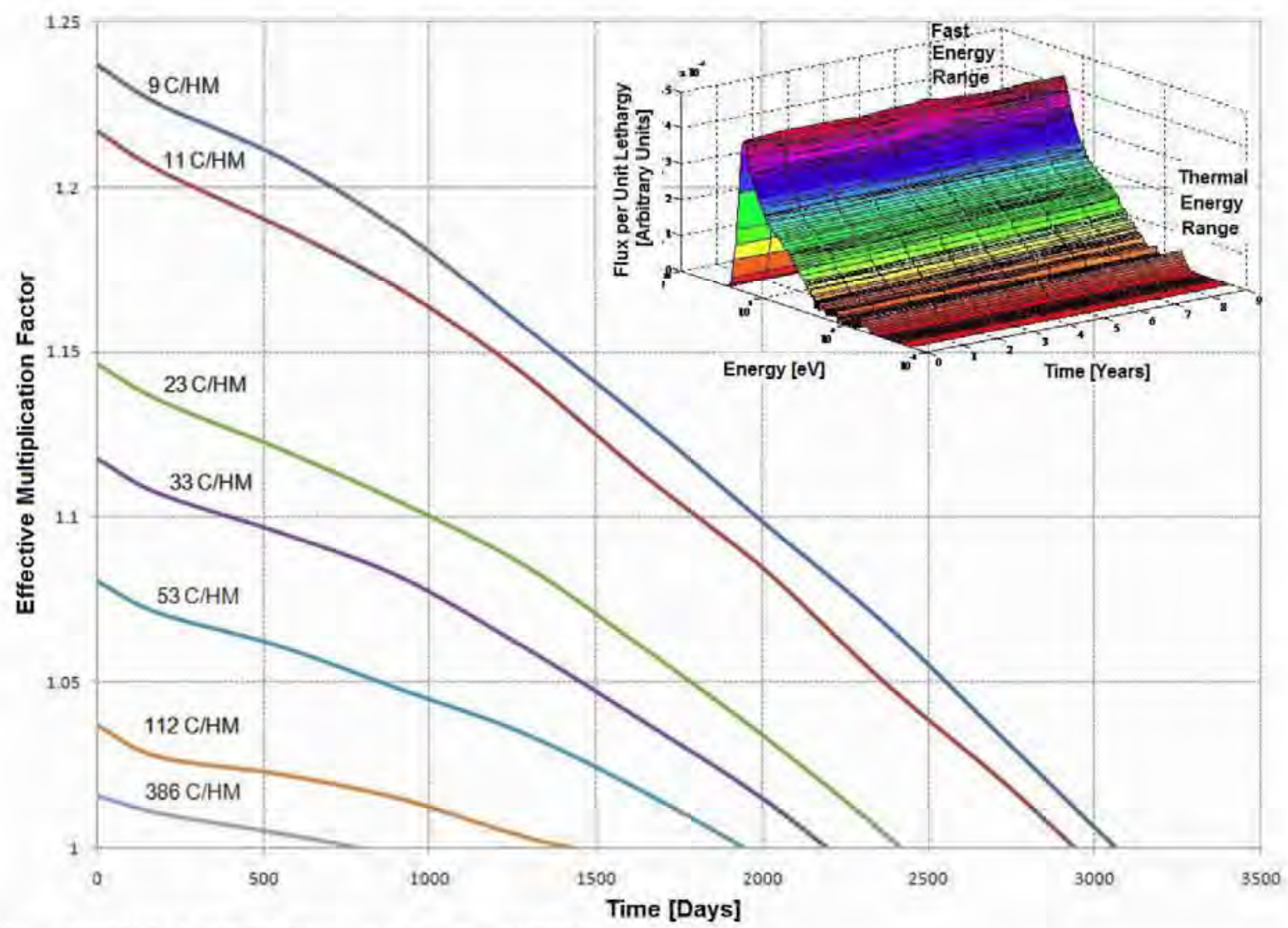

Figure 4. TRU-fueled VHTR lifetime as a function of $\mathrm{C} / \mathrm{HM}$.

In these studies, all considered TRU-fieled VHTR configurations were analyzed assuming their operation at 103 MW/MTHM. As shown in Fig 4, the core lifetimes up to 9 years are possible if attained fast fluence levels can be tolerated by TRISO particles. Furthermore, by simply adjusting the $\mathrm{C} / \mathrm{HM}$ atom ratio inside a compact, the corresponding compact adjustment can extend core lifetime from less than thee years to nine years. Figure 5 shows the fast fluence levels attained by TRISO particles in the LEU- and TRU-fiteled VHTR configurations with the longest lifetimes with respect to criticality. According to Fig. 5, the highest fast fluence level is approximately $7 \times 10^{22} \mathrm{n} / \mathrm{cm}^{2}$. Therefore, expected fast lluences in the longest-operating VHTR configurations approach and may potentially exceed the traditional TRISO fast fluence limitations of about $10^{26} \mathrm{n} / \mathrm{m}^{2},[2,7,12]$ These results clearly indicate the need for radiation-tolerant materials to facilitate a single-batch mode extended operation without refueling. Advanced coating configurations and materials like $\mathrm{ZrC}$ offer promising performance characteristics including reductions of coating failure probabilities yielding fission product releases. $[11,12]$ Consequently, higher fast fluence limits, and hence longer core lifetimes, may become acceptable approachung criticality core lifetime limitations. [5] 


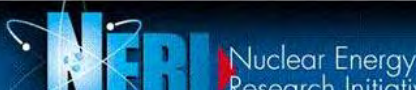 \\ Research Initiative}

Utilization of MAs as a Fuel Component for Ultra-Long Life VHTR Configurations: Designs, Advantages and Limitations

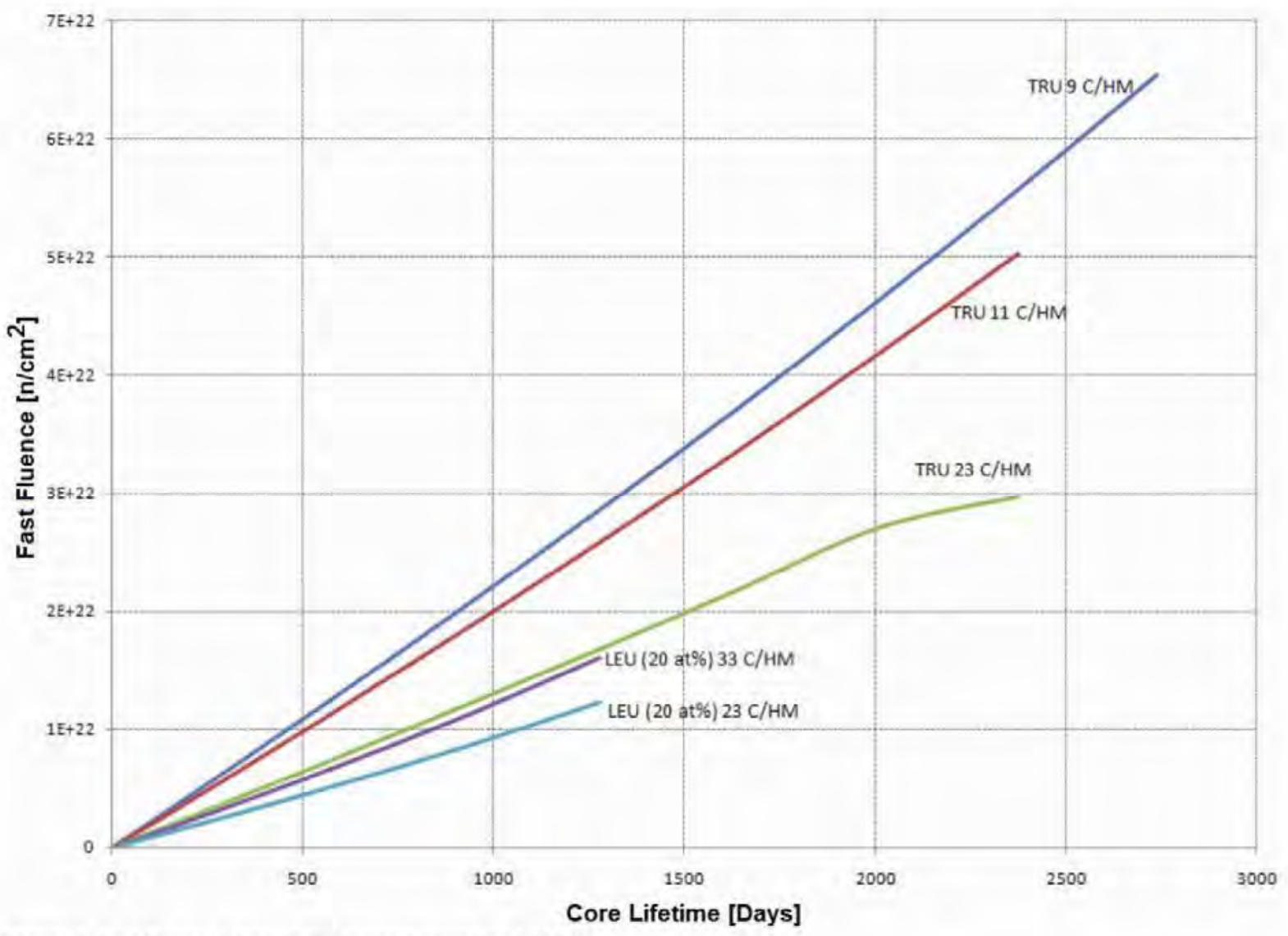

Figure 5. Fast fluence levels in the longest-operating VHTRs.

\section{POTENTIAL VARIATIONS OF TRU VECTORS}

Figure 6 illustrates potential variations of TRU-vectors due to some anticipated variations of the PWR performance characteristics. These variations will ultimately affect the TRUfieled VHTR performance characteristics.

The corresponding equilibrium neutron balance is shown in Fig. 7. The neutron balance calculations for systems with thermal and fast neutrons indicated excess neutrons for all considered TRU-vectors. The thermal neutron balance shows increasing values with increasing scalar flux. In the fast spectrum, the TRU vectors showed a small variation in neutron balance as scalar flux increases.

\section{CONCLUSIONS}

As presented in this paper, the studies of TRU-fueled VHTR configurations indicate promising performance characteristics. The symbiotic quasi-sustainable PWR-VHTR system has been established. Its feasibility and performance characteristics will be explored further in future studies.

The up-to-date results have been analyzed and compared taking into account the existing systems and the Generation IV VHTR concepts with estimated characteristics. Substantial differences between the traditional LEU- and advanced TRUfueled VHTR configurations have been observed.

Evidently, the use of TRU-vectors in VHTRs leads to prolonged single-loading lifetimes. Nine year core-lifetimes were found to be possible for single-batch IRU-fueled VHTR configurations without intermediate refueling. Four year corelifetimes were found to be possible for single-batch LEUfueled VHTR configurations without intermediate refueling. The attained fast fluence levels represent a significant challenge and a strong indication of the need for radiationtolerant materials to facilitate a single-batch mode extended operation without refueling. 
Utilization of MAs as a Fuel Component for Ultra-Long Life

VHTR Configurations: Designs, Advantages and Limitations

Project 05-094

Final Scientific/Technical Report

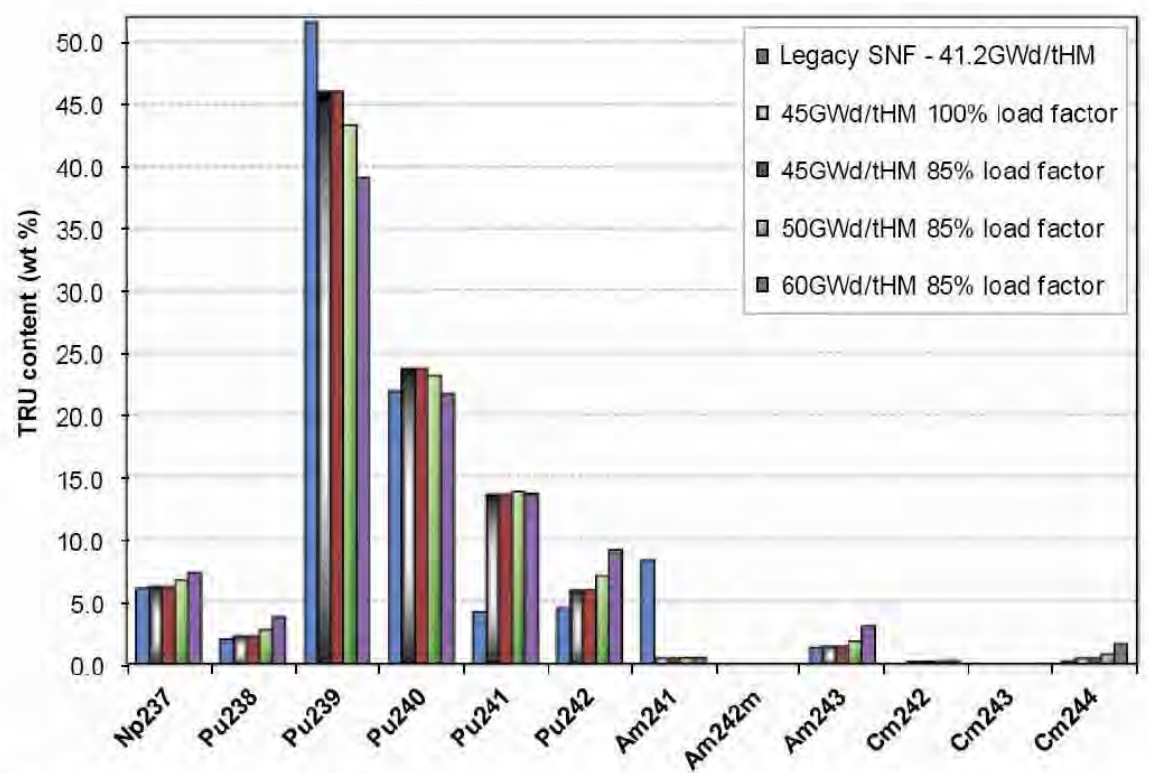

Figure 6. Variations of TRU-vectors due to PWR data.
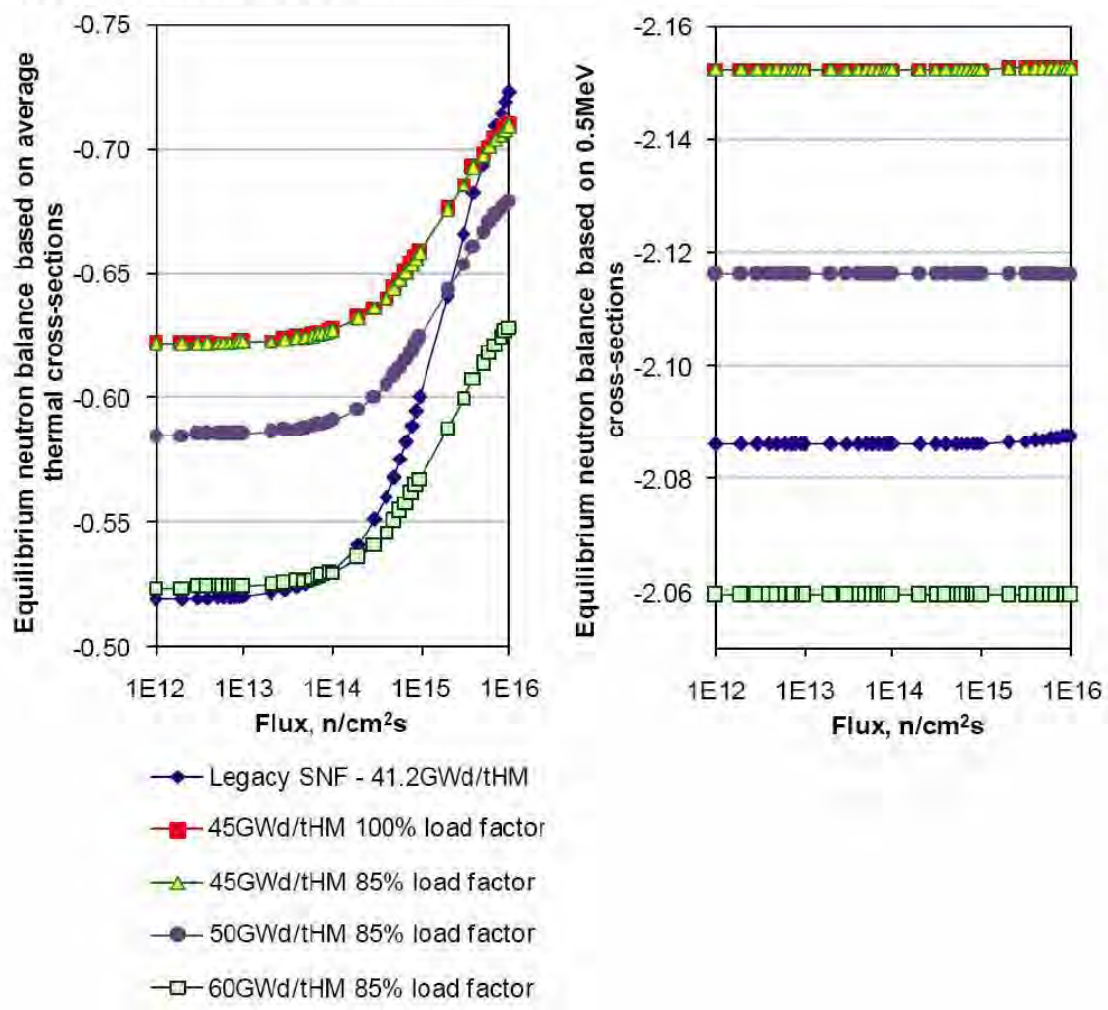

Figure 7. Equilibrium neutron balance in the TRU-systems. 


\section{Utilization of MAs as a Fuel Component for Ultra-Long Life} VHTR Configurations: Designs, Advantages and Limitations

\section{ACKNOWLEDGMENTS}

This paper is based upon work supported by the U.S. Department of Energy under Award Number DE-FC07. 05ID14655 (05-094).

\section{DISCLAIMER}

This paper was prepared as an account of work sponsored by an agency of the United States Goverument. Neither the United Stares Government nor any agency theteof, nor any of their employees, makes any waranty, express or implied, or assumes any legal liability or responsibility for the accuracy. completeness. or usefulness of any information. apparatus. product. or process disclosed. or represents that its use would nor infringe privately owned rights. Reference herein to any specific commercial product. process, or service by trade name. trademark, manufacturer, or otherwise does not necessarily constitute or imply its endorsement, recommendation. or favoring by the United States Government or any agency thereof. The views and opinions of authors expressed herein do not necessarily state or reflect those of the United States Government or any agency thereof.

\section{REFERENCES}

[1] United States Department of Energy, Office of Nuclear Energy, Science, and Technology, 2003, "Report to Congress on Advanced Fuel Cycle Initiative: The Future Path for Advanced Spent Fuel Treatment and Transmutation Research". 03-GA50439-06, U.S. DOE.

[2] C. Rodriguez, A. Baxter, D. McEachern, M. Fikani, F. Venneri, 2003, "Deep-Burn: Making Nuclear Waste Transmutation Practical", Nucl. Eng. Design, 2805, 1.

[3] T. Kim, T. Taiwo, R. Hill, W. Yang, F. Venneri, 2005, "A Feasibility Study of Reactor-Based Deep-Burn Concepts". ANL-AFCI-155, Argonne National Laboratory.

[4] United States Department of Energy, Office of Nuclear Energy, Science, and Technology, 2005, "Generation IV Nuclear Energy Systems: Ten Year Program Plan", March 2005, Office of Advanced Nuclear Researcl. U.S. DOE.

[5] T. Lewis, D, Ames, P. Tsvetkov, 2007, "Analysis of TRU. Fueled VHTR Prismatic Core Performance Domains", Trans. Amer: Soc, 97, 859

[6] International Atomic Energy Agency, 2003, "Evaluation of High Temperature Gas-Cooled Reactor Performance: Benchmark Analysis Related to Initial Testing of the HTTR and HTR-10", IAEA-TECDOC-1382, IAEA, Vienna, Austria.

[7] International Atomic Energy Agency, 2001. "Critical Experiments and Reactor Physics Calculations for LowEuriched HTGRs". IAEA-TECDOC-1249, IAEA, Viemua, Austria
[8] R. Plukiene, D. Ridikas, 2003, "Modeling of HTRs with Monte Carlo, from a Honnogeneous to an Exact Heterogeneous Core with Microparticles", An. Nucl, En. 30. 1573 .

[9] "Final Environmental Impact Statement for a Geologic Repository for the Disposal of Spent Nuclear Fuel and High-Level Radioactive Waste at Yucea Mountain. Nye County, Nevada", 2002. 2. A. "Inventory and Characteristics of Spent Nuclear Fuel, High-Level Radioactive Waste, and Other Materials," DOE/EIS-0250. US. DOE.

[10] Oak Ridge National Labotatory, 2006, "SCALF" a Modular Code System for Performing Standardized Computer Analyses for Licensing Evaluation". ORNL TM-2005/39, Ver, 5,1, ORNL

[11]K. Minato T. Ogawa, K Sawa A Ishikawa, T. Tomita, S. Iida, H. Sekino, 2000, "Irradiation Experiment on $\mathrm{ZrC}$ Coated Fuel Particles for High Temperature Gas-Cooled Reactors", Nucl. Techn., 130, 272

$[12] \mathrm{K}$. Minato, K. Sawa, I. Koya, T. Tomita, A. Ishikawa, 2000, "Fission Product Release Behavior of Individual Coated Fuel Particles for High Temperature Gas-Cooled Reactors", Nucl. Techn., 131. 36. 


\title{
6.5. Out-of-Core Fuel Cycle Characteristics of VHTRs with No On-Site Refueling
}

\author{
Pavel V. Tsvetkov, Ayodeji B. Alajo, Tom G. Lewis III, and David E. Ames II \\ Department of Nuclear Engineering, Texas A\&M University \\ 129 Zachry Engineering Center, MS 3133 TAMU, College Station, TX, 77843-3133 \\ Tel: (979) 845-7078, Fax: (979) 845-6443,Email: Tsvetkov@tamu,edu
}

\begin{abstract}
This paper presents an analysis of VHTR systems with no on-site refueling. The objective of the research effort is to evaluate out-of-core fuel cycle characteristics focusing on potential front-end composition variations and their impact on back-end management options. Recognizing fuel cycle flexibility of HTGRs, a broad range of fresh fuel vectors is taken into consideration including traditional LEU and transmutation fuels containing TRUs from PWR spent fuel. In this analysis, the primary purpose of added TRUs is to achieve prolonged operation on a single fuel loading. Researchers assessed perturbations of the VHTR spent fuel characteristics (back-end management options) because of the VHTR fresh fuel composition deviations from the chosen reference content. A single-batch autonomous operation (no on-site refueling) offers a number of advantages facilitating deployment in energy-demanding developing regions with limited industrial infrastmicture while maintaining system safety and security by design. The study is performed for the hexagonal block core concept within the framework of the ongoing U.S. DOE NERI Project on utilization of TRUs as a fuel component for extended-life VHTRs.
\end{abstract}

\section{INTRODUCTION}

Multiple studies have already demonstrated that hightemperature gas-cooled reactors (HTGRs) and their Generation IV extensions, very-high temperature reactors (VHTRs), have encouraging flexibility with respect to fuel cycle choices. ${ }^{1,2}$ Incineration of TRUs and a single-batch mode operation without intermediate refueling can be achieved in TRU-fueled VHTR configurations. ${ }^{3}$

This paper presents an analysis of out-of-core fuel cycle characteristics of the TRU-fueled VHTR systems with no on-site refueling (once-through-then-out operation assuming that entire reactor modules will be removed and replaced without on-site fuel handling). The objective of the research effort is to evaluate potential front-end composition variations and their impact on back-end management options.

As mentioned above, recognizing fuel cycle flexibility of HTGRs, a broad range of fresh fuel vectors is taken into consideration including traditional LEU and transmutation fuels containing TRUs from PWR spent fuel. In this analysis, the primary purpose of added TRUs is to achieve prolonged operation on a single fuel loading. Researchers assessed perturbations of the VHTR spent fuel characteristics (back-end management options) because of the VHTR fresh fuel composition deviations from the chosen reference content. A single-batch autonomous operation (no on-site refueling) offers a number of advantages facilitating deployment in energy-demanding developing regions with limited industrial infrastructure while maintaining system safety and security by design.

The study is performed for the hexagonal block core concept within the framework of the ongoing U.S. DOE NERI Project on utilization of TRUs as a fuel component for extended-life VHTRs. ${ }^{3}$ The TRU-fueled VHTR prismatic core configurations have inherent flexibility of component configuration, fuel utilization, and fuel management. As will be illustrated in this paper, flexibility in the component configuration allows for the carbon-toheavy metal $(\mathrm{C} / \mathrm{HM})$ atom ratio to be adjusted. The $\mathrm{C} / \mathrm{HM}$ adjustments shift neutron spectra towards higher energies. Harder spectra, in the case of TRU fuels, result in selfstabilization effects potentially extending operation without intermediate refueling.

These systems are developed and analyzed accounting for controllability, dynamics and safety margins, and proliferation-resistance during the entire reactor lifetime. The resulting configurations should have inherent 


\section{Utilization of MAs as a Fuel Component for Ultra-Long Life} VHTR Configurations: Designs, Advantages and Limitations

\section{Project 05-094}

\section{Final Scientific/Technical Report}

capabilities for utilization of TRUs from spent LWR fuel. Consequently, successful deployment of partitioning and transmutation (P\&T) of TRUs and their further utilization in VHTRs facilitate incineration of these hazardous nuclides and could potentially provide additional fuel supply.

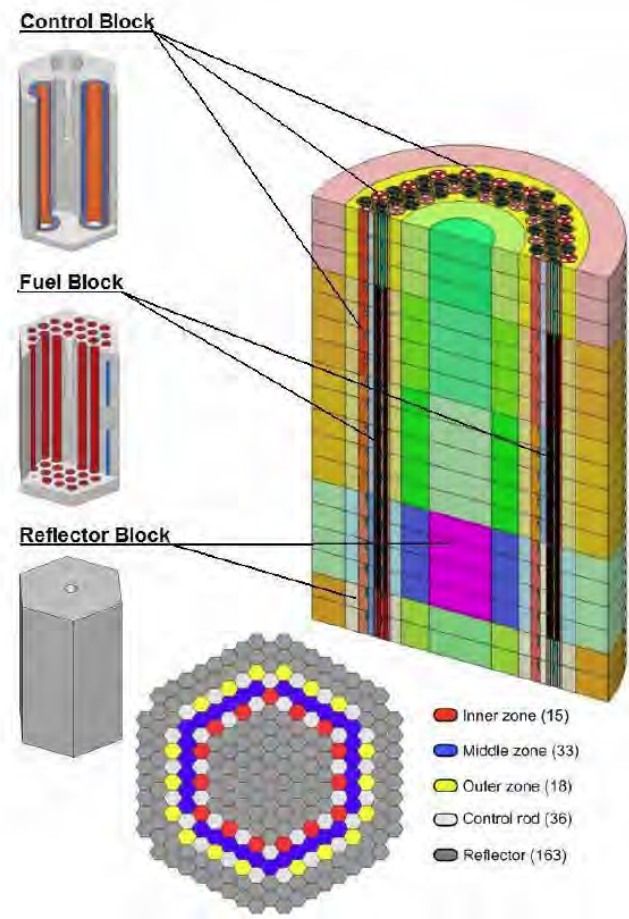

Fig. 1. 3D whole-core exact-geometry VHTR model.

Behavior of the advanced nuclear energy systems like Generation IV VHTRs is difficult to predict due to inherent safety features resulting in tight coupling between energy production, transport and conversion, and system behavior during normal operation and off-normal situations. The suggested integrated/hybrid Monte-Carlo-deterministic modeling approach offers the desirable analysis capabilities for the VHTR design studies. The use of Monte Carlo codes creates a potential for explicit 3D whole-core/reactor studies of the VHTR configurations addressing the key reactor physics areas such as the double and multi-level heterogeneity, neutron streaming in the low-density regions, and neutron spectrum transitions at the interfaces. ${ }^{3}$

\section{APPLIED COMPUTER CODE SYSTEM}

The 3D whole-core exact-geometry VHTR model has been created for use with SCALE 5.1 sequences focusing on KENO V.a. at the whole-core modeling level. ${ }^{4}$ The model is shown in Fig. 1.

As illustrated in Fig. 1, the model is a near exact representation of the expected physical description of the VHTR configuration. The computational scheme combines the available capabilities of the SCALE Monte Carlo (whole-core analysis level) and deterministic functional modules (lattice level).

\section{MULTI-HETEROGENEITY OF VHTR CORES}

The use of coated micro-particles creates doubleheterogeneity that defines performance characteristics of the VHTR core. The first level of heterogeneity (microlevel) is formed by coated fuel micro-particles that are randomly distributed in the graphite compact. The second level of heterogeneity (macro-level) is formed by the prismatic blocks with compacts that compose the VHTR core. The prismatic graphite block core has channels for coolant, instrumentation and handling operations. As a result, there are various neutron streaming passages formed by coolant channels, instrumentation and handling channels, plenums, and etc. The proximity to outer and central reflector regions is also an important factor that influences the VHTR physics. As emphasized in Fig. 2, there are substantial differences in neutron distributions at each heterogeneity level. Depending on a specific computational model, these features act and can be taken into account as multiple additional levels of heterogeneity up to the $3 \mathrm{D}$ finite core model that provides an appropriate comprehensive multi-heterogeneity treatment.

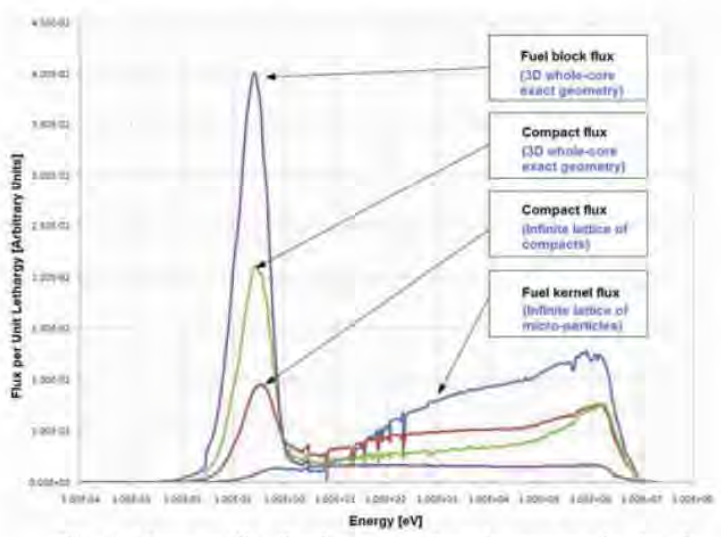

Fig. 2. Neutron distributions at various heterogeneity levels.

Each compact has a fuel region containing thousands of micro-particles that form a "universe". The resonance self-shielding effects are determined by the microscopic flux behavior in the fuel, which strongly depends on the geometry of the fuel configuration. As a result, the resonance absorption should be calculated on the basis of

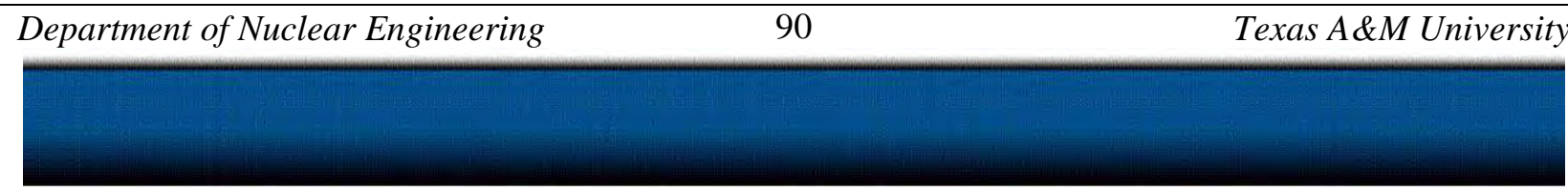




\section{Utilization of MAs as a Fuel Component for Ultra-Long Life VHTR Configurations: Designs, Advantages and Limitations}

\section{Project 05-094}

the microscopic lattice of coated fuel micro-particles. Because the neutron mean free path in this lattice is comparable to the dimensions of a compact, the lattice of coated fuel micro-particles cannot be considered infinite. Outer particles feel compact boundaries, coolant channels and graphite within a hexagonal block as well as the presence of neighboring compacts. This makes probabilities of neutron interactions with fuel kernels space-dependent within a compact. Furthermore, as illustrated in Fig. 2, the core neutron distribution is formed by neutron media within each compact and then at the block and at the whole core levels accounting for various neutron streaming effects.

\section{VALIDATION AND VERIFICATION}

To assure comprehensive, realistic assessment of the VHTR designs and their performance characteristics targeting passive safety confirmation, the adequacy of the applied computational methods and models used to evaluate performance must be supported by comparisons with experimental data covering appropriate ranges of conditions. Validation data are available from power reactors as well as past critical experiments

As the basis for the benchmark test problems, the project uses the actual test results and benchmark problems of the HTTR Program (prismatic core design, reactor experiments). ${ }^{5,6}$

\section{SPECTRAL EFFECTS OF C/HM-VARIATIONS}

The VHTR prismatic core configurations offer significant flexibility in component configuration, fuel utilization and management. It should be possible to enhance properties of some TRUs by neutron spectrum shifting through configuration adjustments. In particular, the use of TRISO-coated micro-particles allows for variable $\mathrm{C} / \mathrm{HM}$ ratios. Fig. 3 shows how the TRU-fueled VHTR can have its neutron spectrum changed by adjusting the $\mathrm{C} / \mathrm{HM}$ atom ratio.

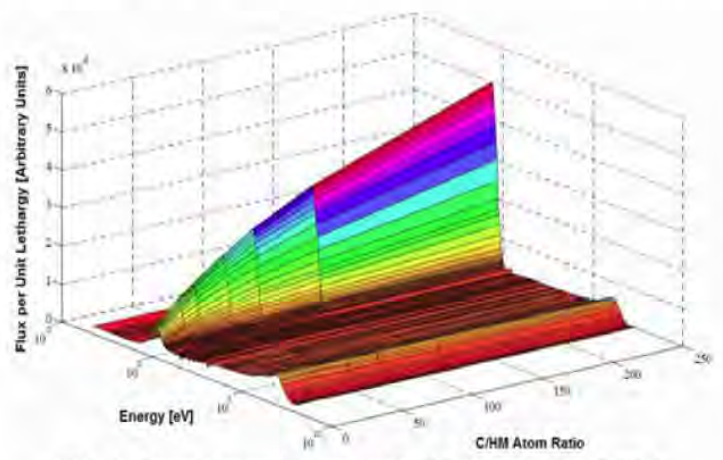

Fig. 3. Spectrum variations in the VHTRs due to $\mathrm{C} / \mathrm{HM}$.

\section{Final Scientific/Technical Report}

The principal mechanism being envisioned to achieve extended-life systems is an enhanced involvement of selfgenerated fissile compositions based on LWR spent fuel vectors to the energy generation process. Depending on neutron spectra in the considered core configurations (dominant neutron energies), neptunium, americium and curium may serve as burnable poisons or fuel materials contributing to self-stabilization over prolonged irradiation periods.

\section{IN-CORE FUEL CYCLE}

Figure 4 illustrates the TRU-fueled VHTR performance during extended operation for considered TRU vectors:

- TRU vectors of the legacy spent nuclear fuel (SNF) after decades of cooling, PWR, 41.2 $\mathrm{GWd} / \mathrm{tHM}, 23$ years cooling,

- TRU vectors of the PWRs with $100 \%$ load factors, $45 \mathrm{GWd} / \mathrm{tHM}$,

- TRU vectors of the PWRs with $85 \%$ load factors, $45 \mathrm{GWd} / \mathrm{tHM}$,

- TRU vectors of the PWRs with $85 \%$ load factors, $50 \mathrm{GWd} / \mathrm{tHM}$,

- TRU vectors of the PWRs with $85 \%$ load factors, $60 \mathrm{GWd} / \mathrm{tHM}$,

- Reference LEU vectors.

The reduction of fissile content is significant for the $20 \%$ TRU-fueled VHTRs.

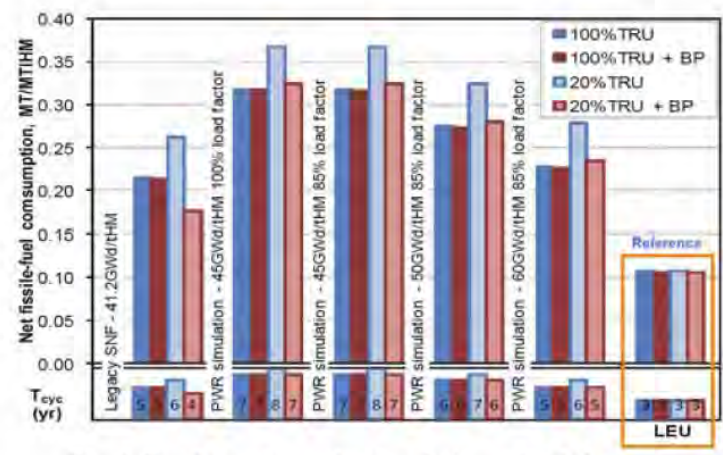

Fig. 4. Fissile content reduction during extended operation.

The LEU-fueled configuration utilizes about $75 \%$ of the initially loaded fissile content over 3 year operation. All TRU-fueled configurations demonstrate smaller reductions over much longer lifetimes.

\section{OUT-OF-CORE FUEL CYCLE}

Figure 5 shows the TRU-fueled VHTR out-of-core fuel cycle performance characteristics with respect to the reference LEU-cycle. The analysis accounts for relative 


\section{Utilization of MAs as a Fuel Component for Ultra-Long Life VHTR Configurations: Designs, Advantages and Limitations}

\section{Project 05-094}

transmutation efficiency, TRU destruction rate, and TRU disposal rate.

The reference LEU-fueled VHTR discharges about $87 \%$ of its initial heavy metal loading. All TRU-fueled VHTRs discharge smaller quantities of their initial heavy metal loadings. The resulting transmutation efficiencies are shown in Fig. 5.

When the VHTR spent fuel is expected to be sent to the Yucca Mountain Geological Repository, it is going to be assessed on two factors. First, before a waste form can enter the subsurface environment, the waste package has to fall below a specific heat emission requirement. The second and the most important criterion is activity of the HLW. For the TRU-fueled VHTR spent fuel to reach the same activity per fuel volume as in the initial loading, the required cooling time is about 10 years.

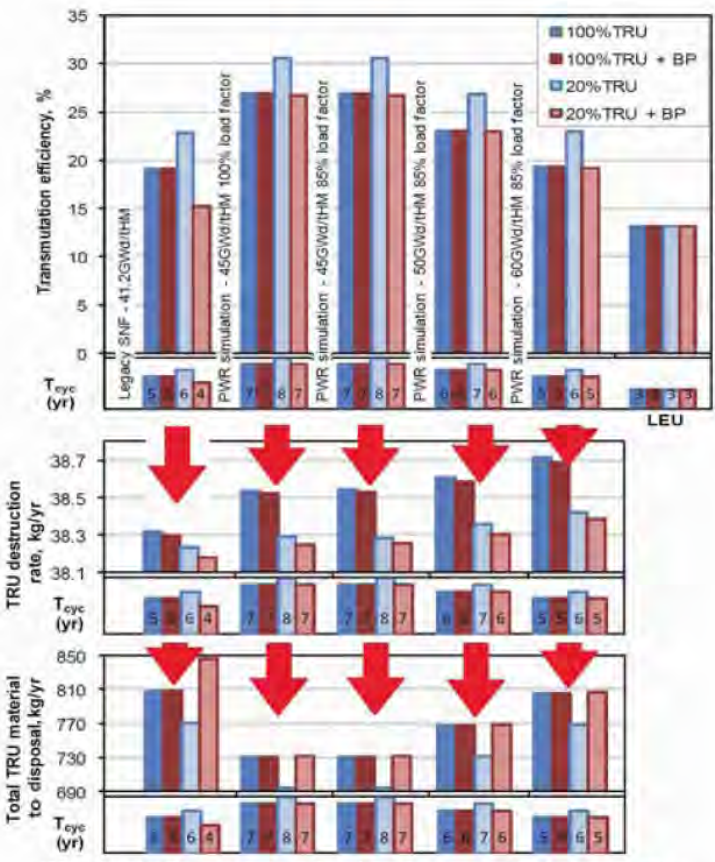

Fig. 5. Fissile content reduction during extended operation.

\section{CONCLUSIONS}

The research effort enhances capabilities of the Generation IV VHTR and potentially transforms it to a technology that can deliver electricity, hydrogen, and assist in spent nuclear fuel management. It has been determined that core lifetimes of about 8 years are achievable in the TRU-fueled VHTR configurations whereas the LEUsystems remain operational in a single-batch mode only for about 3 years without refueling. The corresponding destruction rates are about $30 \%$. At the same time,
Final Scientific/Technical Report

radiotoxicity levels in nuclear waste streams require certain cooling times to drop below initial levels.

\section{ACKNOWLEDGMENTS}

This paper is based upon work supported by the U.S. Department of Energy under Award Number DE-FC0705ID14655 (05-094).

\section{DISCLAIMER}

This paper was prepared as an account of work sponsored by an agency of the United States Government. Neither the United States Government nor any agency thereof, nor any of their employees, makes any warranty, express or implied, or assumes any legal liability or responsibility for the accuracy, completeness, or usefulness of any information, apparatus, product, or process disclosed, or represents that its use would not infringe privately owned rights. Reference herein to any specific commercial product, process, or service by trade name, trademark, manufacturer, or otherwise does not necessarily constitute or imply its endorsement, recommendation, or favoring by the United States Government or any agency thereof. The views and opinions of authors expressed herein do not necessarily state or reflect those of the United States Government or any agency thereof.

\section{NOMENCLATURE}

C/HM - Carbon-to-Heavy Metal Atom Ratio

DOE - U.S. Department of Energy

HLW - High Level Wastes

HTGR - High Temperature Gas-cooled Reactor

LEU - Low-Enriched Uranium

LWR - Light Water Reactor

MA - Minor Actinides

NERI - Nuclear Energy Research Initiative

P\&T - Partitioning and Transmutation

PWR - Pressurized Water Reactor

SNF - Spent Nuclear Fuel

TRISO - Tristructural Isotropic Coating

TRU - Transuranium Nuclides

VHTR - Very High Temperature Reactor

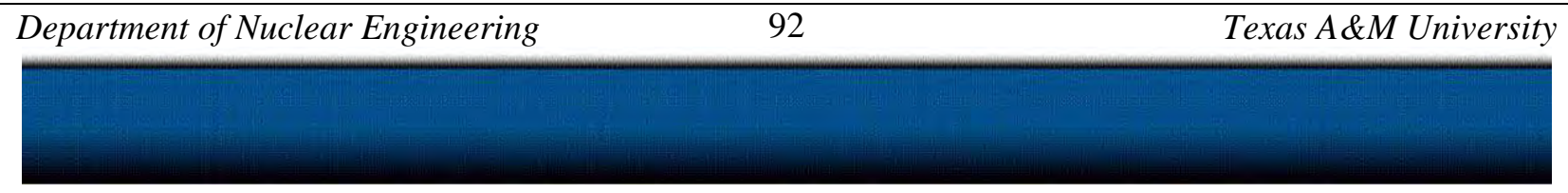


Utilization of MAs as a Fuel Component for Ultra-Long Life VHTR Configurations: Designs, Advantages and Limitations

1. "Report to Congress on Advanced Fuel Cycle Initiative: The Future Path for Advanced Spent Fuel Treatment and Transmutation Research", 03GA50439-06, January, 2003, Office of Nuclear Energy, Science, and Technology, U.S. DOE (2003).

2. "Generation IV Nuclear Energy Systems: Ten Year Program Plan", March 2005, Office of Advanced Nuclear Research, DOE Office of Nuclear Energy, Science, and Technology, U.S. DOE (2005).

3. T. LEWIS, D. AMES, P. TSVETKOV, "Analysis of TRU-Fueled VHTR Prismatic Core Performance Domains", Trans. Amer. Soc, 97, 859 (2007).

4. "SCALE: A Modular Code System for Performing Standardized Computer Analyses for Licensing Evaluation", ORNL/TM-2005/39, Ver. 5.1, April 2006, Oak Ridge National Laboratory (2006).

5. "Critical Experiments and Reactor Physics Calculations for Low-Euriched HTGRs", IAEATECDOC-1249, International Atomic Energy Agency, Vienna, Austria (2001)

6. "Evaluation of High Temperature Gas-cooled Reactor Performance: Benchmark Analysis Related to Initial Testing of the HTTR and HTR-10", IAEA-TECDOC1382, International Atomic Energy Agency, Vienna, Austria (2003).

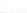


Utilization of MAs as a Fuel Component for Ultra-Long Life VHTR Configurations: Designs, Advantages and Limitations

Project 05-094

Final Scientific/Technical Report

\subsection{Utilization of TRUs in VHTRs - Operation in a Single-Batch Mode: Front End, Back End, and Performance}

Pavel V. Tsvetkov, Tom G. Lewis III, Ayodeji B. Alajo

Department of Nuclear Engineering, Texas A\&M University, 129 Zachry Engineering Center, MS 3133 TAMU College Station, TX, 77843-3133,E-mail:tsvetkov@tamu.edu

\section{INTRODUCTION}

The eurrently accepted waste management strategy for spent nuclear fuel (SNF) presumes disposing highlevel nuclear wastes (HLW) in geological repositories. [1] Recognizing the need for a long-term strategy assuring nuclear power sustainability, ongoing efforts on advanced closed-fuel cycle options and challenges in opening and safeguarding the Yucca Mountain repository have led to investigations of various strategies involving utilization of transuranic (TRU) nuclides in nuclear reactors. Due to favorable performance characteristics, multiple studies focus on transmutation options using High Temperature Gas-cooled Reactors (HTGRs). [2,3]

The objective of the present analysis is to assess performance characteristics of TRU-fueled VHTRs operating in a single-batch mode. The focus is on their front-end and back-end fuel cycle characteristics and system performance, especially their ability to approach a prolonged operation mode without intermediate refueling.

This twofold strategy would make use of fuels containing mansuranic (TRU) nuclides in nuclear reactors. and would allow marketing power units with VHTRs as nuclear batteries for worldwide deployment. The analysis is performed within the framework of the 3-year U.S. DOE NERI project that targets design envelops of TRUfueled VHTRs operating in an extended batch mode without refueling. [3]

\section{LEU-LWR-SNF-TRU-VHTR-FP CYCLE CONCEPT}

Figure 1 shows the envisioned LWR-VHTR fuel cycle with TRU-recycle that is designed to yield only fission products for disposal in the geological repository. In this scheme, PWR fivel elements with $3.75^{\circ}$ LEU, which were bumt up to $41,200 \mathrm{MWd}$ mtHM and cooled for 23 years, are being reprocessed. [4] Partitioned TRU and depleted uranim (DU) are then recycled in VHTRs (continuous lines) and potentially in PWRs (dashed line). When fast reactors (FR) become viable, the suggested fuel cycle scheme may be transfonmed into a sustamable nuclear energy system that does not require a LEU-feed.

It is expected that LWRs will eventually be replaced by Generation IV VHTRs. At that time, sustainable nuclear systems consisting of FRs and VHTRs will become possible.

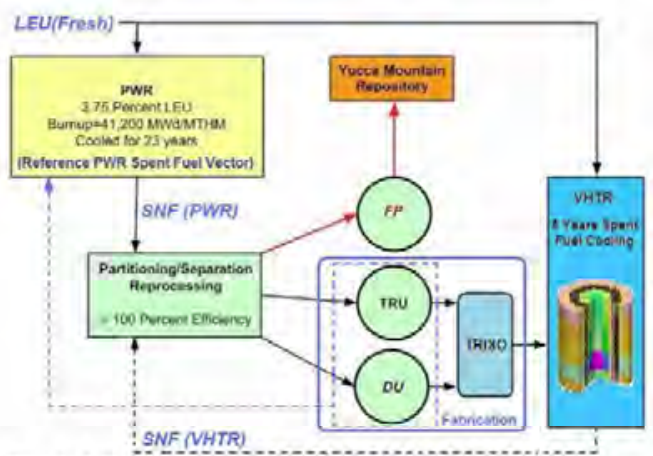

Fig. 1. LWR-VHTR fuel cycle with TRU-recycle.

\section{HIGH-FIDELITY REACTOR MODEL}

A 3-D whole-core exact-geometry model of a VHTR hexagonal-block configuration with a detailed temperature distribution was developed and implemented for calculations with the ORNI. SCALE 5.1 code system.[5] The applied VHTR model is shown in Fig. 2.

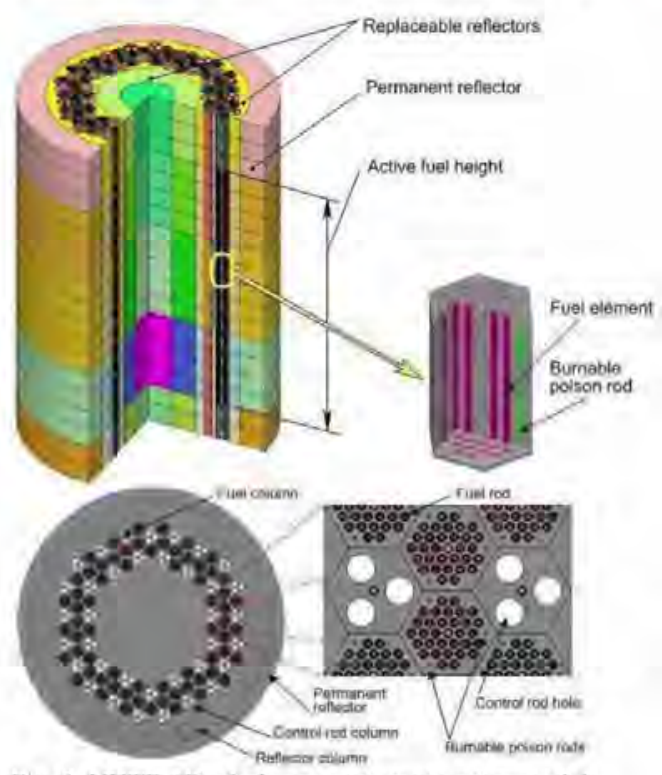

Fig, 2. VHTR 3D whole-core exact-geometry model 


\section{Utilization of MAs as a Fuel Component for Ultra-Long Life} VHTR Configurations: Designs, Advantages and Limitations

In this analysis, the modeling adequacy has been confirmed in extensive series of experiment-to-code benchmark evaluations. [6]

\section{PERFORMANCE ANALYSIS}

Transitioning from LEU-systems to VHTR without refueling up to almost a decade-long batch mode that is limited by radiation damage effects. Figure 3 illustrates: configurations with TRUs allows prolonging operation

- spectral differences between LWRs and VHTRs, and between VHTRs fueled with LEU and TRUs;

- flexibility of VHTRs to prolong operation without refueling and minimize excess reactivity levels by adjusting carbon-toheavy metal atom ratio.

Comparing to VHTRs with LEU, spectral transitions during operation are significantly smaller in VHTRs with TRUs.

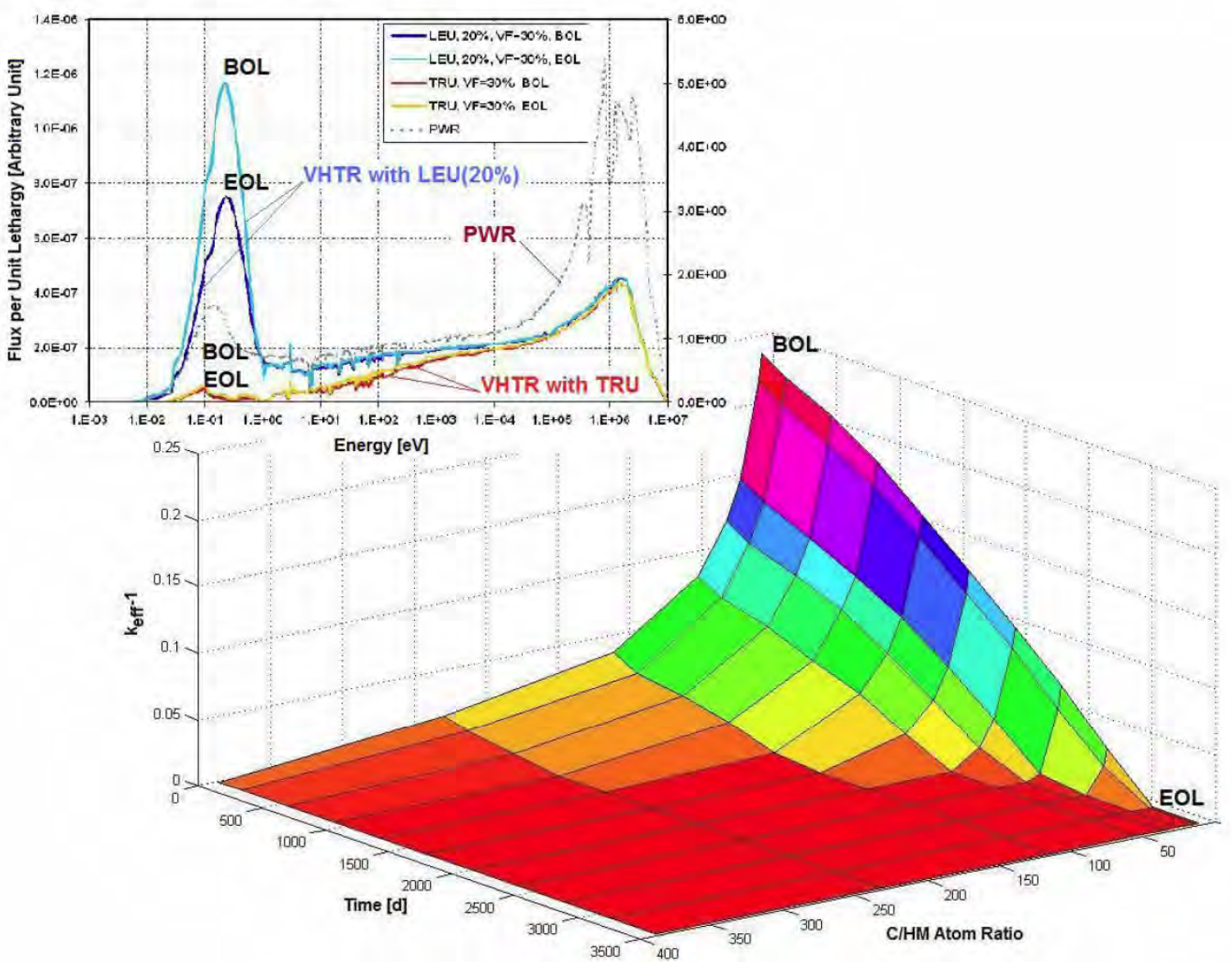

Fig. 3. Spectral transitions in VHTRs due to fuel composition variations and configuration adjustments to extend operation and minimize excess reactivity levels.

The corresponding end-of-life (EOL) fractions of TRU-destroyed in VHTRs are shown in Table I. For the TRU-fueled VHTR spent fuel, it will take approximately 10 years of cooling time to reach the BOL activity level. Table II compares EOL fast fluence levels attained in the comparable LEU- and TRU-fueled VHTR configurations.
TABLE I. C/HM Ratios and TRU Destruction in VHTRs

\begin{tabular}{|l|l|l|}
\hline C/HM Atom Ratio & Lifetime (y) & $\begin{array}{l}\text { EOL/BOL TRU } \\
\text { Destruction (\%) }\end{array}$ \\
\hline 53 & 6.0 & 22.21 \\
\hline 112 & 5.0 & 18.44 \\
\hline 229 & 2.0 & 7.36 \\
\hline
\end{tabular}


Utilization of MAs as a Fuel Component for Ultra-Long Life VHTR Configurations: Designs, Advantages and Limitations

TABLE II. Fluences in LEU- and TRU-Fueled VHTRs

\begin{tabular}{|l|l|l|}
\hline Fuel & Lifetime (y) & EOL Fast Fluence $\left(\mathbf{n} / \mathbf{c m}^{2}\right)$ \\
\hline $20 \%$ TRU & 4.0 & $2.72 \mathrm{E}+21$ \\
\hline LEU & 3.0 & $7.76 \mathrm{E}+21$ \\
\hline
\end{tabular}

\section{CONCLUSIONS}

The analysis has shown that HTGRs and their Generation IV extensions, VHTRs, have encouraging performance characteristics. Results of extensive criticality and depletion calculations with multiple fuel loadings (TRU vectors) demonstrated achievability of a single-batch extended-lifetime mode. Radiotoxicity of the TRU-fueled VHTR EOL vectors were found to require a decade-long cooling to reach radiotoxicity levels of the $\mathrm{BOL}$ vectors.

\section{ACKNOWLEDGMENTS}

This paper is based upon work supported by the U.S. Department of Energy under Award Number DE-FC0705ID14655 (05-094).

\section{REFERENCES}

1. "Report to Congress on Advanced Fuel Cycle Initiative: The Future Path for Advanced Spent Fuel Treatment and Transmutation Research", 03GA50439-06, U.S. DOE (2003).

2. T. KIM, T. TAIWO, R. HILL, W. YANG, F. VENNERI, "A Feasibility Study of Reactor-Based Deep-Burn Concepts", ANL-AFCI-155, Argonne National Laboratory (2005)

3. T. LEWIS, D. AMES, P. TSVETKOV, "Analysis of TRU-Fueled VHTR Prismatic Core Performance Domains", Trans. Amer. Soc., 97, 859 (2007).

4. "Final Environmental Impact Statement for a Geologic Repository for the Disposal of Spent Nuclear Fuel and High-Level Radioactive Waste at Yucca Mountain, Nye County, Nevada", 2, A, "Inventory and Characteristics of Spent Nuclear Fuel, High-Level Radioactive Waste, and Other Materials," DOE/EIS-0250, U.S. DOE (2002).

5. "SCALE: A Modular Code System for Performing Standardized Computer Analyses for Licensing Evaluation", ORNL/TM-2005/39,Ver.5.1, November 3, 2006, Oak Ridge National Laboratory, Tennessee (2006).

6. P. TSVETKOV, A. ALAJO, D. AMES, M. PRITCHARD, "Spectrum Shifting as a Mechanism to Improve Performance of VHTRs with Advanced Actinide Fuels", Proc. 14th Intern. Conf. Nucl. Eng. (ICONE 14), Miami, Florida, July 17 - 20, 2006 , ASME (2006). 


\title{
6.7. Reactor Physics of VHTRs with $\mathrm{UO}_{2}$, $\mathrm{UCO} \& \mathrm{UC}_{0.5} \mathrm{O}_{1.5}$ Kernels Operating without Onsite Refueling
}

\author{
Ayodeji B. Alajo, Pavel V. Tsvetkov
}

Nuclear Engineering Department, Texas A\&M University, MS 3133 TAMU, College Station, Texas, 77843

Email: dejialajo@tamu.edu

\section{INTRODUCTION}

This analysis is focused on Very High Temperature Reactor (VHTR) performance characteristics considering uranium oxycarbides and uranium oxides for oncethrough single-batch operation. The uranium oxycarbide fuel kernels exhibit properties, which make them desirable as one of the potential fuel options. They are capable to perform well at high burnup levels and are also known to inhibit carbon monoxide formation in the TRISO carbon coatings, thus lowering the potential of pressure related failures in the TRISO particles. Similar to oxide fuels, the oxycarbides efficiently retain fission products including those that can compromise the integrity of the coating layers [1]

These studies have been performed within the framework of the 3-year U.S. DOE NERI project that targets a variety of performance aspects of VHTRs operating in an extended batch mode without refueling.

\section{THE VHTR CORE MODEL}

A 3-D whole core prismatic VHTR in ammular configuration was explicitly modeled with the ORNL SCALES.1 code system [2]. The fuel assembly blocks were based on the High Temperature Test Reactor (HTTR) fuel blocks [3]. The adequacy of the core models has been confirmed in a series of experiment-to-code benchmark evaluations [4].

Fuel depletion calculations were performed with the T5-DEPL sequence [5]. The 238-Group ENDF/B-VI nuclear data library was used for all SCALE computations. Kernels with $\mathrm{UO}_{2}, \mathrm{UCO}$ and $\mathrm{UC}_{05} \mathrm{O}_{15}$ have been considered at $15 \%{ }^{235} \mathrm{U}$ enrichment. The calculations included cases with and without $\mathrm{B}_{4} \mathrm{C}$ burnable poison (BP). All calculations were performed at system temperature of $1223 \mathrm{~K}$. Several metrics were applied to assess performance characteristics:

- Fraction of the total fissile material fissioned

- Total Minor Actinide (MA) material to dispose

- Helium production

\section{PERFORMLANCE ANALYSIS}

To illustrate VHTR operation history without refueling, Fig. 1 shows the time dependent effective multiplication factor in the VHTR core. The criticality trends in the oxycarbide fueled cores are similar to the oxide fueled core. The VHTR model resulted in supereritical systems with all fuel compositions. The suppression of multiplication factor by the BP is effective within the first 600 days of operation. For all considered cases, the core reached sub-criticality after 900 days.

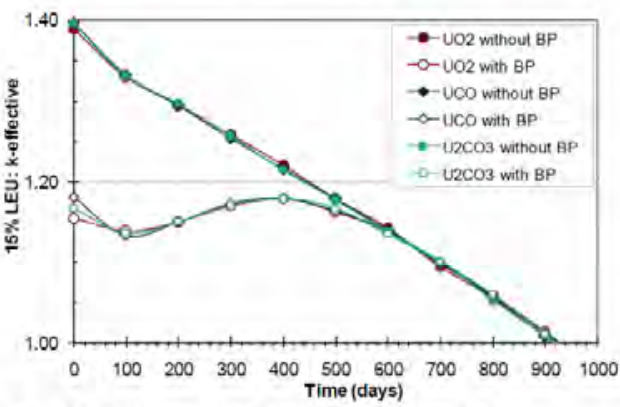

Fig. 1. VHTR operation history without refueling.

It has been observed that more fissile materials were depleted in cases without BP compared with corresponding case with BP. The difference in the fraction of fissile content depleted between cases with and without BP ranges between $0.54 \%$ and $0.6 \%$ of initial fissile loading.

Figure 2 shows the MA content of the spent fiel after 900 days of irradiation followed by 25 years of cooling. The produced quantities of MAs are similar irrespective of fuel kernel types. There are notable increases in MA content between discharged quantities at the $900^{\text {th }}$ day and at $25^{\text {th }}$ year of cooling. The increased MA content after cooling is mainly due to the decay of ${ }^{241} \mathrm{Pu}$ to ${ }^{241} \mathrm{Am}$. Excluding ${ }^{241} \mathrm{Am}$, net MA content reduced after decay. The reduction is due to $\alpha$-decay of ${ }^{241} \mathrm{Cm}$.

Helium production in oxycarbide fuel is less than in oxide fuel. Helium production appears to reduce with increasing carbon content in the fuel kernel. It is however not expected that the helium content will contribute significantly to the pressure in the kernel since the fission gases content in the kernel are significantly higher than helium. With fuel discharge at $900^{\text {th }}$ day, krypton and xenon contents in the spent fuel are about $1.2 \mathrm{~kg}$ and $14.0 \mathrm{~kg}$ respectively for all fuel types. The corresponding helium content at the same discharge time is less than $4 \times 10^{-3} \mathrm{~kg}$. 


\section{Utilization of MAs as a Fuel Component for Ultra-Long Life} VHTR Configurations: Designs, Advantages and Limitations

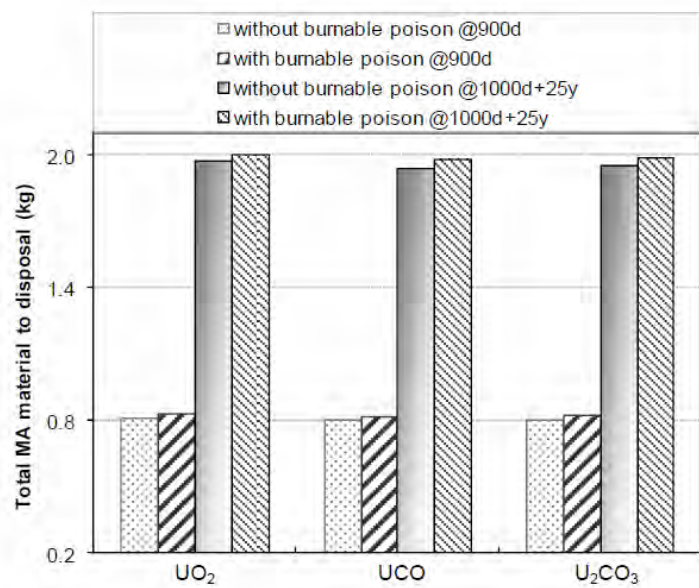

Fig. 2. MA materials to be disposed after 900irradiation days and 25 year cooling.

\section{CONCLUSIONS}

As a result of the performed analysis, it has been observed that the core lifetime is not sensitive to the chosen fuel type. Fissile fuel utilization is comparable amongst the considered fuels. There is no obvious advantage in one fuel type over the other from the stand point of MA radiotoxicity reduction since MA discharge levels are about the same for all considered fuel types. The reduced helium production is a positive result for the oxycarbide fuel; however it does not represent a clear advantage in fuel performance.

\section{ACKNOWLEDGMENTS}

This paper is based upon work supported by the U.S. Department of Energy under Award Number DE-FC0705ID14655 (05-094).

\section{REFERENCES}

1. M.B. RICHARDS et.al. "H2-MHR Pre-conceptual Design Report: SI-Based Plant," GA-A25401, General Atomics, San Diego, USA (2006).

2. "SCALE: A Modular Code System for Performing Standardized Computer Analyses for Licensing Evaluation," ORNL/TM-2005/39,Ver.5.1, Oak Ridge National Laboratory, Tennessee (2006).

3. T. IYOKU, S. UETA, J. SUMITA, M. UMEDA and M. ISHIHARA, "Design of core components," Nuclear Engineering and Design, 233, 71-79 (2004).

4. P. TSVETKOV, A. ALAJO, D. AMES, M. PRITCHARD, "Spectrum Shifting as a Mechanism to Improve Performance of VHTRs with Advanced Actinide Fuels", Proc. 14th Intern. Conf. Nucl. Eng. (ICONE 14), Miami, Florida, July 17 - 20, 2006, ASME (2006).

5. M. D. DEHART, "TRITON: A Two-Dimensional Transport and Depletion Module for Characterization of Spent Nuclear Fuel," Vol. I, Book 3, Sect. T1 of SCALE: A Modular Code System for Performing Standardized Computer Analyses for Licensing Evaluation, ORNL/TM-2005/39, 3 Volumes (2006). 


\title{
6.8. Core lifetime and fuel utilization in prismatic VHTR cores
}

\author{
Ayodeji Alajo $^{\mathrm{a}, *}$, Pavel Tsvetkov ${ }^{\mathrm{a}, * *}$ \\ ${ }^{a}$ Texas A\&M University, College Station, USA
}

\begin{abstract}
The Very High Temperature Reactor (VHTR) is a candidate for next generation nuclear plant (NGNP). Accumulated experience with high temperature gas-cooled reactors makes the VHTR the most near-term of all Gen-IV reactors. The VHTR configurations with varying transuranic nuclide (TRU) compositions were analyzed assuming a single-batch core operation. The considered TRU compositions were derived from simulated PWR spent fuel compositions. The 3D models of a prismatic VHTR core were created for use in calculations with the SCALE 5.1 code system. The TRU fuel performance in VHTR prismatic configurations was compared with low enriched uranium (LEU) fueled VHTRs. The analyses showed that TRUs can be effectively employed as VHTR fuel. The TRU-fueled VHTRs exhibited longer core lifetimes and better fuel utilization than the LEU-fueled VHTR.
\end{abstract}

\section{Background}

The work performed was inspired by international and United States of America programs such as Generation IV Energy Systems Initiative (Gen-IV) and Global Nuclear Energy Partnership (GNEP).

\subsection{Nuclear energy initiatives}

The Generation IV program is an international collaborative effort established by the US Department of Energy (DOE) to develop the next generation nuclear energy systems that will broaden the opportunities for the use of nuclear energy. Based on the requirements of the Generation IV

\footnotetext{
* Student contact: Ayodeji Alajo, dejialajo@tamu.edu, Tel: +1 979845 4161; Fax: +1 9798456443 .

** Advisor contact: Pavel Tsvetkov, tsvetkov@tamu.edu Tel: +1 979845 7078; Fax: +19798456443.
}

roadmap, the VHTR and five other reactor designs were selected as the Generation IV system.

Another initiative by the United States Department of Energy (DOE) led to the Global Nuclear Energy Partnership (GNEP), which is aimed at increasing U.S. and global energy security, while reducing nuclear proliferation risks. The GNEP envisions closed fuel cycle development by nations with advanced nuclear capabilities. The closed fuel cycle technology development and deployment would enable consumption of TRU, employing new proliferation-resistant technologies.

\subsection{The VHTR}

The Very High Temperature Reactor (VHTR) is based on technologies that have been well

\begin{tabular}{lll}
\hline Department of Nuclear Engineering & 99 & Texas A\&M University \\
\hline &
\end{tabular}




\section{Utilization of MAs as a Fuel Component for Ultra-Long Life} VHTR Configurations: Designs, Advantages and Limitations

established in High Temperature Gas Reactors (HTGRs), such as German AVR and THTR prototypes, and the U.S. Fort Saint Vrain and Peach Bottom prototypes. The VHTR extends the capabilities of HTGRs to achieve further improvements in thermal efficiency and open up additional high-temperature applications.

The VHTR is a graphite-moderated heliumcooled reactor that supplies heat at core outlet temperatures above 850 degrees Celsius. The fuel design is characterized by the use of TRISO (TRIstructural ISOtropic) coated fuel particles. The TRISO fuel provides self-containment for each fuel particle. A porous carbon layer provides buffer for fuel kernel swelling and fission gas migration. A second layer of high density pyrolitic carbon coat prevents contact between a layer of strong irradiation resistant coating and the fuel kernel. The irradiation resistant layer of coating allows complete retention of fission product under reactor operation conditions.

The prismatic core configuration consists of fuel assembly blocks, reflector blocks and control rod guide blocks. The core is formed by arranging these blocks in an annular layout. All blocks are hexagonal and made of graphite. The fuel assembly block houses the fuel elements, which are composed of annular fuel compacts stacked in a graphite sleeve. The fuel compacts feature TRISO particles embedded in graphite matrix.

\section{Objective}

The objective of the current work is to provide an initial analysis of variations in TRU fuel compositions and their impacts on core lifetime estimates and TRU-fuel utilization characteristics for VHTR cores. The current effort is focused on prismatic core configuration operated under a single batch once-through fuel cycle option. Incineration of TRUs in the VHTR is also evaluated through fuel utilization.

Alternative approach for the potential use of TRUs in HTGRs is represented by General Atomics "deep-burn" concept that is based on utilization of thermal neutrons and high burn-up fuels in modular helium reactor (MHR) systems. The concept is focused on TRU s from LWR spent fuels. An essential factor in this approach is the use of ceramic-coated fuels to achieve high TRU destruction levels (Kim et al., 2005; Rodriguez et al., 2003). The deep-burn concept uses multibatch reloading and recycling schemes.

\section{Methodology}

Unlike the deep-burn concept, method in this effort is focused on TRU-fueled VHTRs and their performance characteristics during operation in a single-batch mode without refueling. In order to achieve the stated objective, the following tasks were performed.

- Determination of TRU vector for VHTR fuel. This task involved the use of IAEA's Nuclear Fuel Cycle Simulation System (VISTA) to determine potential PWR spent fuel compositions. Additional composition was determined from the analysis of United States legacy spent fuel that is given in the Yucca Mountain Safety Assessment Report.

- Development of a detailed whole-core 3-D model of the prismatic VHTR. The fuel assembly block model was based on Japan's HTTR fuel block configuration. The reactor fuel loadings were based on the PWR spent fuel vectors identified in the preceding task. The model development and implementation was accomplished through the use of ORNL SCALE 5.1 code system.

- Analysis of the TRU-fueled VHTR systems. The analyses focused on the effects of variations in fresh TRU-based fuel compositions on VHTR fuel utilization and core lifetime.

- To establish a reference reactor system, calculations for LEU-fueled VHTR were performed and the results were used as the basis for comparative studies of the TRUfueled systems. The LEU fuel is uranium oxide at $15 \%{ }^{235} \mathrm{U}$ enrichment.

Five TRU fuel compositions were selected: four from VISTA simulations and the fifth from the analysis of Yucca Mountain Safety Assessment Report. The selected compositions are:

- TRU1: Legacy spent nuclear fuel: average burnup of 41.2 GWd/MTHM, 23 year cooling time.

- TRU2: PWR spent fuel at $45 \mathrm{GWd} / \mathrm{tHM}$ burnup and $100 \%$ load factor.

- TRU3: PWR spent fuel at $45 \mathrm{GWd} / \mathrm{tHM}$ burnup and $85 \%$ load factor.

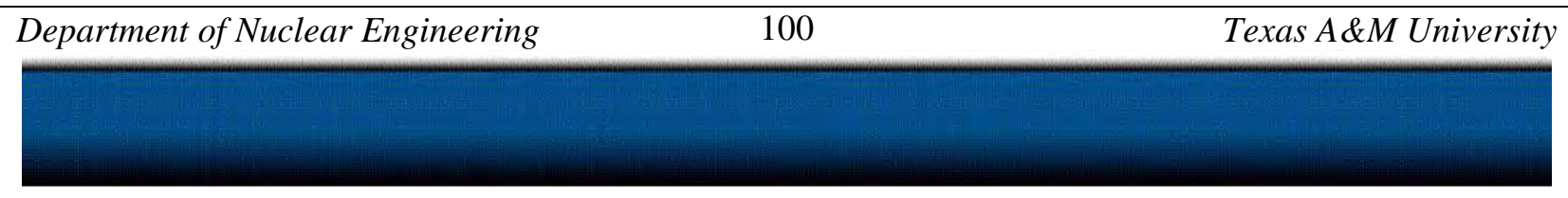




\section{Utilization of MAs as a Fuel Component for Ultra-Long Life VHTR Configurations: Designs, Advantages and Limitations}

- TRU4: PWR spent fuel at $50 \mathrm{GWd} / \mathrm{tHM}$ burnup and $85 \%$ load factor.

- TRU5: PWR spent fuel at $60 \mathrm{GWd} / \mathrm{tHM}$ bumup and $85 \%$ load factor.

Each TRU vector represented a fuel composition - the $100 \%$ TRU cases. Additional fuel compositions of $20 \%$ TRU- $80 \%$ Carbon were developed from each TRU vector. Uranium oxide fuel at $15 \%$ enrichment was the reference fuel for in-core performance analysis. These gave a total of 11 different fuel kernel compositions that were covered in the analyses. All fuel kernel densities were fixed at $10.41 \mathrm{~g} / \mathrm{cc}$. All fuel compositions were analyzed under scenarios with and without burnable poison.

The metrics developed and employed in the VHTR analysis were of two categories:

- Metrics for the in-core fuel cycle studies which evaluated core lifetime considering system multiplication factor and fast fluence limit.

- Metrics for the out-of-core fuel cycle studies - which evaluated fuel utilization over the life of the core. The transmutation efficiency of the core was evaluated.

The fast fluence $\Phi_{f}$ was determined using the basic formula:

$$
\Phi_{f}=\phi_{f, a v g} T=\phi_{f, a v g} \Sigma\left(t_{i}\right)
$$

where $\phi_{f, \text { avg }}$ is the average fast flux in the system, $t_{i}$ is the time interval at which flux data were calculated and $T$ is the total operation time of the core before it attains subcriticality. The average fast flex was evaluated as:

$$
\phi_{f, a v g}=\Sigma\left(\phi_{f, i} t_{i}\right) / \Sigma\left(t_{i}\right)
$$

The parameter $\phi_{f, i}$ defines the fast flux evaluated at interval $t_{i}$ of the core's operation time. The fast fluxes at the intervals, $\phi_{f, i}$ were determined as:

$$
\phi_{f, i}=\phi_{T, i}-\phi_{t h, i}
$$

Where $\phi_{T, i}$ and $\phi_{t h, i}$ are the total flux and thermal fluxes after time interval $t_{i}$. The evaluation of the fast flux is conservative since it includes epithermal flux. The equations (1) to (3), when combined, give the final equation for fast fluence as:

$$
\Phi_{f}=\Sigma\left[\left(\phi_{T, i}-\phi_{t h, i}\right) t_{i}\right]
$$

The transmutation efficiency $\varepsilon_{T}$ was calculated using the formula:

$$
\varepsilon_{T}=100\left(N_{i}-N_{o}\right) / N_{i}
$$

The parameters $N_{i}$ and $N_{o}$ are the total initial TRU loaded and the total TRU discharged at end of core life.

The initial TRU loading was user predetermined from PWR spent fuel compositions, while all other input parameters to the above equations were obtained from SCALE 5.1 calculations.

\section{Results and discussion}

The use of TRUs in VHTRs led to super-critical cores (see Table 1) with longer lifetimes and lower start-up multiplication factors than in the corresponding LEU cases. The results indicate promising performance characteristics for TRUbased fuels in VHTRs.

Table 1

Beginning of life (BOL) multiplication factors $\left(\mathrm{k}_{\text {eff }}\right)$ for VHTR cores with and without burnable poison (BP) ${ }^{\mathrm{a}, \mathrm{b}}$

\begin{tabular}{lll}
\hline Case & $\begin{array}{l}\text { BOL keff } \\
100 \% \mathrm{TRU}\end{array}$ & $\begin{array}{l}\text { BOL } \mathrm{k}_{\text {eff }} \\
20 \% \mathrm{TRU}\end{array}$ \\
\hline TRU1 & 1.0785 & 1.0309 \\
\hline TRU2 & 1.2461 & 1.1427 \\
\hline TRU3 & 1.2482 & 1.1404 \\
\hline TRU4 & 1.2331 & 1.1337 \\
\hline TRU5 & 1.1982 & 1.1080 \\
\hline TRU1 w/BP & 1.1127 & 1.1124 \\
\hline TRU2 w/BP & 1.2879 & 1.2281 \\
\hline TRU3 w/BP & 1.2866 & 1.2279 \\
\hline TRU4 w/BP & 1.2714 & 1.2209 \\
\hline TRU5 w/BP & 1.2363 & 1.2039 \\
\hline LEU & 1.1546 & \\
\hline LEU w/BP & 1.3886 & \\
\hline The notation 'w/BP' implies core with burnable poison. \\
$\begin{array}{l}\text { Cases without the notation are cores without burnable } \\
\text { poison. }\end{array}$ \\
$\begin{array}{l}\text { The LEU multiplication factors on the Table are for } \\
100 \% \text { LEU fuel cores. }\end{array}$
\end{tabular}




\section{Trpice \\ Research Initiative}

\section{Utilization of MAs as a Fuel Component for Ultra-Long Life VHTR Configurations: Designs, Advantages and Limitations}

\subsection{Core lifetime}

Fig. 1 shows the time dependent multiplication factors and fast fluence at the end of core lifetime for each fuel composition. The core lifetime was determined as the year before the VHTR core becomes subcritical. The primary limiting factor for the core lifetime is a fast fluence limit of $5 \times 10^{21} \mathrm{n} / \mathrm{cm}^{2}$. All cores with $100 \%$ TRU fuel exceed the fast fluence limit before attaining subcriticality. The $20 \%$ TRU cases were within the fast fluence limit with the exception of TRU2 and TRU3 fuels without burnable poison. The 2 exceptions were at the boundary of the imposed limit. Based on the $20 \%$ TRU cases, the core lifetimes ranged between 4 and 8 years. The LEU fuel could only sustain a 3 year core lifetime with the associated fast fluence higher than the $20 \%$ TRU fuel cases.

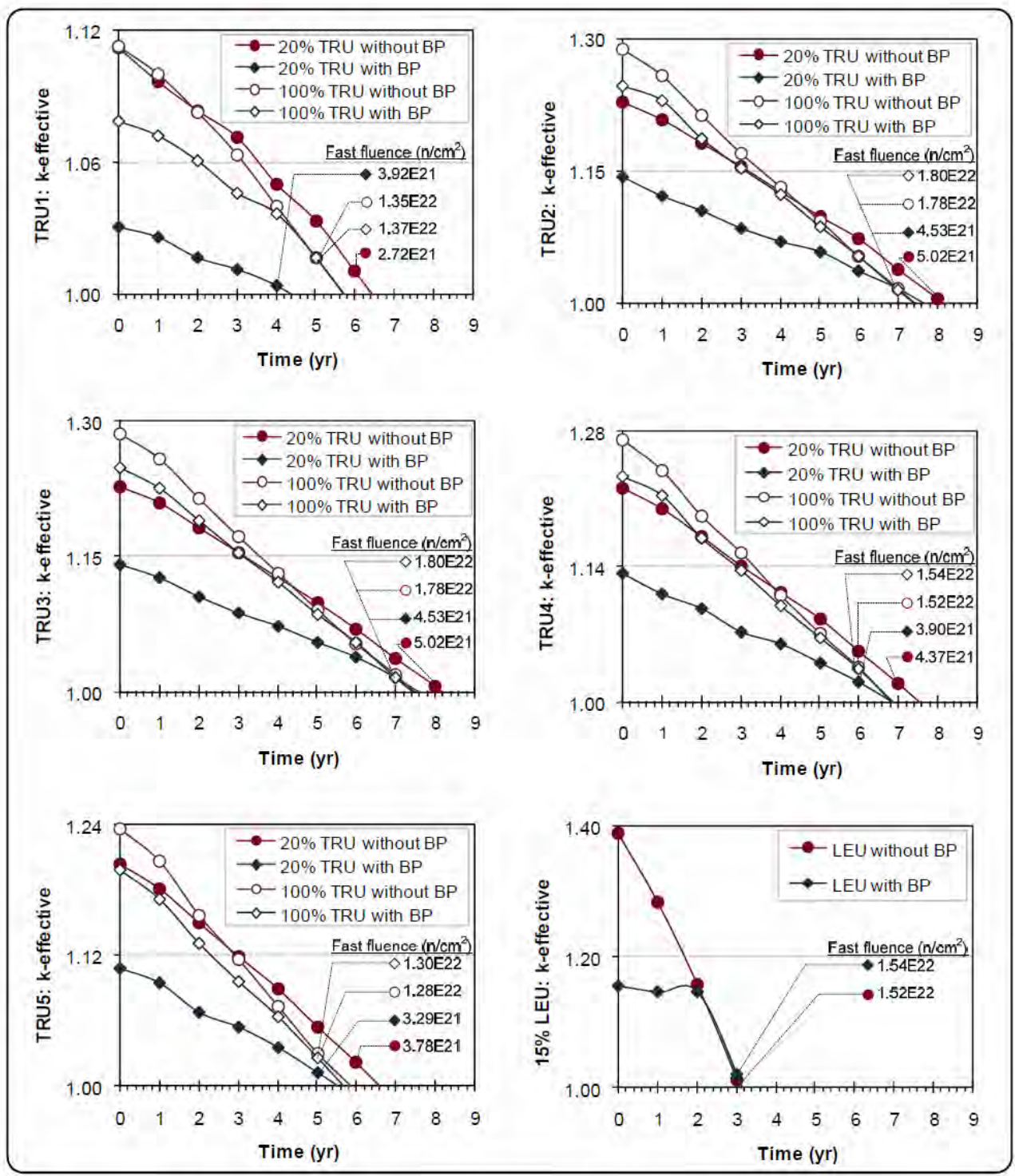

Fig. 1. Time dependent multiplication factor, core lifetime and fast fluence. 


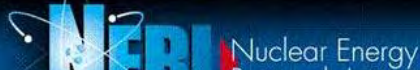

Utilization of MAs as a Fuel Component for Ultra-Long Life VHTR Configurations: Designs, Advantages and Limitations

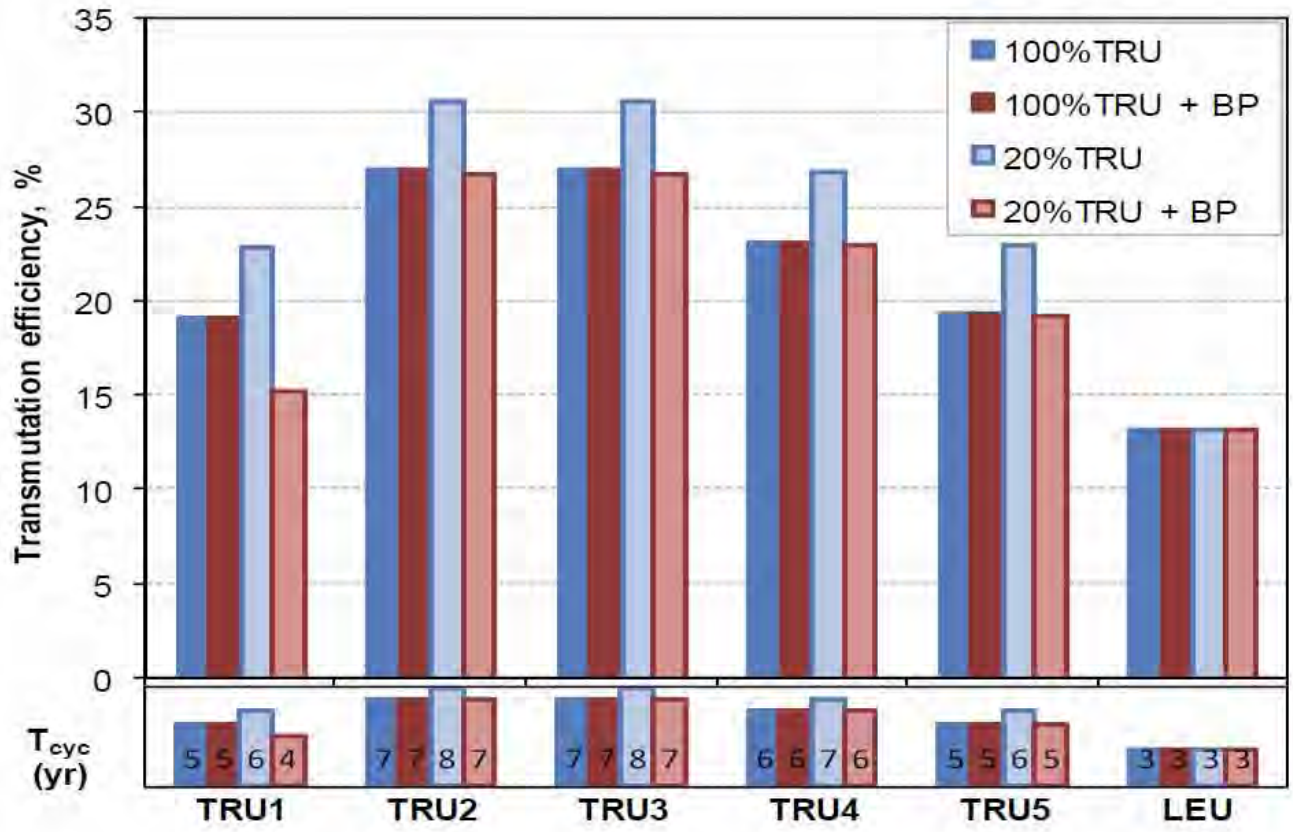

Fig. 2. Transmutation efficiency. The numbers on the lower bars are core lifetimes.

\subsection{Fuel utilization}

All TRU fuels discharged less quantity of heavy metal compared with LEU fuel. This is a further confirmation of the effectiveness of TRU as fiel component. The fraction of initial TRU loaded going to waste stream at discharge ranged between 0.69 and 0.85 for the TRU fueled cores -0.87 in LEU fuel. The addition of burnable poison had negligible effect on discharged quantities for all $100 \%$ TRU cases. In the 20\%TRU fueled cases, addition of burnable poison resulted in increased discharge quantities, which are higher than the $100 \%$ discharge from corresponding $100 \%$ TRU case.

The resulting transmutation efficiencies are provided in Fig. 2. The observed transmutation efficiencies ranged between $15 \%$ and $31 \%$ in TRU fuels $-13 \%$ in LEU fuels. As indicated in the Fig 2, the $20 \%$ TRU cases without burnable poison are more efficient than other cases.

\section{Conclusions}

The analysis of the TRU-fueled VHTR configurations indicated promising performance characteristics (see Figs. 1 and 2). The VHTRs with TRUs showed improved core lifetimes and fuel utilization factors relative to LEU systems. The results also showed that VHTR performance vary as a function of initial TRU fuel compositions. Further improvements may be achieved through further refinements or the core configurations and/or fuel compositions.

\section{Acknowledgement}

This paper is based upon work supported by the U.S. Department of Energy under Award Number DE-FC07-05ID14655 (05-094). 


\section{References}

Iyoku, T., Ueta, S., Sumita, J., Umeda, M., Ishihara, M., 2004. Design of core components. Nuclear Engineering and Design. 233, 71-79.

Kim, T.K., Taiwo, T.A., Hill, R.N., Yang, W.S., Venneri, F., 2005. A feasibility study of reactor-based deep-burn concepts. Nuclear Engineering Division, Argonne National Laboratory, USDOE. ANL-AFCI-155,

Rodriguez, C., Baxter, A., Mceachern, D., Fikani, M., Venneri, F., 2003. Deep-burn: making nuclear waste transmutation practical. Nuclear Engineering and Design. 222, 299317.

2001. Current status and future development of modular high temperature gas-cooled reactor technology. International Atomic Energy Agency, Vienna, Austria. IAEA-TECDOC1198.

2002. Inventory and characteristics of spent nuclear fuel, high-level radioactive waste, and other materials. Appendix A in: Final environmental impact statement for a geologic repository for the disposal of spent nuclear fuel and high-level radioactive waste at Yucca Mountain. Nye County, Nevada. Vol. 2, U.S. DOE DOE/EIS-0250.

2003. Screening tests for selection of VHTR advanced fuel. Issued by General Atomics for the Department of Energy Contract No. DEAC03-01SF22343.

2003. The US Generation IV implementation strategy. Office of Nuclear Energy Science, and Technology, USDOE. 03-GA50439-06.

2007. Global Nuclear Energy Partnership Strategic Plan. GNEP-167312, Rev 0. Office of Nuclear Energy - Office of Fuel Cycle Management. USDOE. 


\title{
6.9. VHTR-Based Systems for Autonomous Co-Generation Applications
}

\author{
Pavel V. Tsvetkov, David E. Ames II, Ayodeji B. Alajo, Tom G. Lewis III \\ Department of Nuclear Engineering \\ Texas A\&M University \\ 129 Zachry Engineering Center, MS 3133 TAMU, College Station, Texas 77843-3133, USA \\ Phone: 979/845-7078, \\ Fax: 979/845-6443 \\ E-mail:Tsvetkov@tamu.edu
}

\section{ABSTRACT}

As highly efficient advanced nuclear systems, Generation IV Very High Temperature Reactors (VHTR) can be considered in a variety of configurations for electricity generation and process heat applications. Simultaneous delivery of electricity, low-temperature process heat (for potable water production, district heating, etc.) and high temperature process heat (for hydrogen production, etc.) by a single cogeneration system offers unique deployment options as "all-in-one" power stations. This paper is focused on the VHTR-based systems for autonomous co-generation applications. The analysis is being performed within the scope of the U.S. DOE NERI project on utilization of higher actinides (TRUs and partitioned MAs) as a fuel component for extended-life VHTR configurations. It accounts for system performance characteristics including VHTR physics features, control options and energy conversion efficiencies. Utilization of TRUs in VHTRs is explored to stabilize in-core fuel compositions (core self-stabilization) leading to extended single-batch OTTO (Once-Through-ThenOut) modes of operation without intermediate refueling.

\begin{tabular}{|c|c|}
\hline NUIVIEN & URE \\
\hline HTGR & High Temperature Gas-cooled Reactor \\
\hline HTTR & High Temperature Test Reactor \\
\hline JAERI & Japan Atomic Energy Research Institute \\
\hline LEU & Low-Enriched Uranium \\
\hline LWR & Light Water Reactor \\
\hline MA & Minor Actinides \\
\hline MOP & Multi-objective Optimization Problem \\
\hline NERI & Nuclear Energy Research Initiative \\
\hline NGNP & - Next Generation Nuclear Plant \\
\hline OTTO & - Once-Through-Then-Out \\
\hline TRU & - Transuranium Nuclides \\
\hline VHTR & Very High Temperature Reactor \\
\hline
\end{tabular}

\section{INTRODUCTION}

As highly efficient advanced nuclear systems, Generation IV Very High Temperature Reactors (VHTR) can be considered in a variety of configurations for electricity generation and process heat applications. $[8,10,18]$ Simultaneous delivery of electricity, low-temperature process heat (for potable water production, district heating, etc.) and high temperature process heat (for hydrogen production, etc.) by a single cogeneration system offers unique deployment options as "all-in-one" power stations.

The basic technology has been well established in High Temperature Gas Reactors (HTGR), such as the German AVR and THTR prototypes, and the U.S. Fort Saint Vrain and Peach Bottom prototypes. The VHTR extends the capabilities of HTGRs to achieve further improvements in thermal efficiency and establish a technological basis for emerging hightemperature applications. [8]

This paper is focused on the VHTR-based systems for autonomous co-generation applications. The analysis is being performed within the scope of the U.S. DOE NERI project on utilization of higher actinides (TRUs and partitioned MAs) as a fuel component for extended-life VHTR configurations. [2428] It accounts for system performance characteristics including VHTR physics features, control options and energy conversion efficiencies. The study is carried out for the hexagonal block core concept.

Flexibility of VHTRs with respect to their fuel cycle choices is one of the well-known features of HTGRs. [25] It is viable to consider PWR spent fuel vectors as being reprocessed and recycled for further utilization as transmutation fuel feeds of VHTRs. Utilization of TRUs in VHTRs is explored to stabilize in-core fuel compositions (core self-stabilization) leading to extended single-batch OTTO (Once-Through-ThenOut) modes of operation without intermediate refueling. [24]

\begin{tabular}{lll}
\hline Department of Nuclear Engineering & 105 & Texas A\&M University \\
\hline &
\end{tabular}




\section{Utilization of MAs as a Fuel Component for Ultra-Long Life VHTR Configurations: Designs, Advantages and Limitations}

Depending on neutron spectra, neptunium, americium and curium may contribute to small reactivity swings (selfstabilization) over prolonged irradiation periods. Under certain spectral conditions TRUs would be able to contribute to the VHTR core neutron balance compensating for fuel depletion effects through their chain transformations, [10,24] As generation III - reactors phase out, the TRU-fueled VHTRs would become a dominant thermal reactor transmutation option taking advantage of unique characteristics of TRUs.

\section{APPLIED CODE SYSTEM}

The analysis methodology has been implemented for coupled design studies of VHTRs with TRUs using the ORNL SCALE 5.1 code system. Newly available in SCALE version 5.1 is the capability to treat multi-heterogeneity. [20] The double-heterogeneity treatment supports quasi-explicit representation of TRISO micro-particles within a compact fuel region. As schematically shown in Fig. 1, the procedure leads to homogenized fuel regions in fuel compacts with properly shielded cross sections.
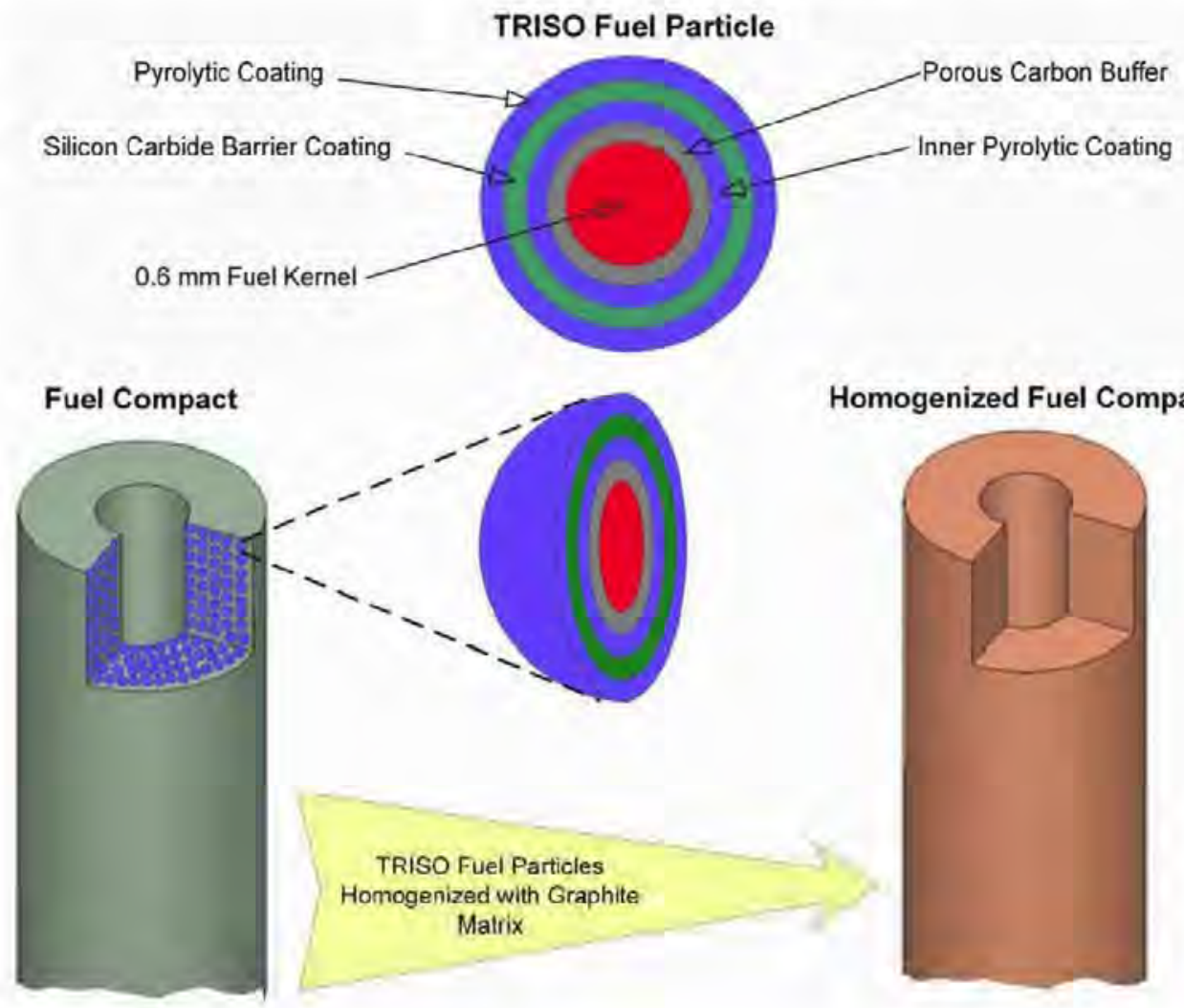

Homogenized Fuel Compact

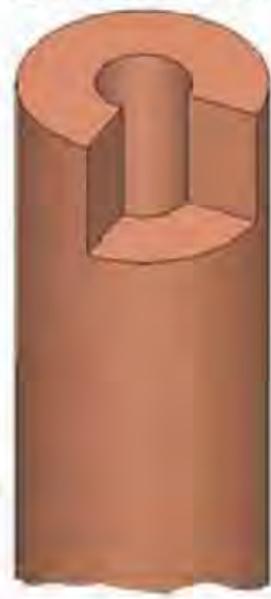

Figure 1, Homogenization of TRISO particles.

Table 1 provides a comparison of the SCALE 5.1 and HTTR benchmark results. [4.21] The key teclmical issues are being addressed and resolved by implementing computationally efficient automated modeling procedures and sequences. combining continuous energy Monte Carlo and multigroup Monte Carlo and determimustic approaches.
Table 1. HTTR Criticality Calculations Using SCALE 5.1

\begin{tabular}{|l|l|l|}
\hline VHIR Model & $k_{\text {edr }}$ & Error $(\%)$ \\
\hline HTIR Benclimurk Data & 1.1363 & - \\
\hline SCALE 5.1 (wifh DOUBLEHET) & 1,1368 & $0.04 \%$ \\
\hline
\end{tabular}




\section{Utilization of MAs as a Fuel Component for Ultra-Long Life} VHTR Configurations: Designs, Advantages and Limitations

\section{VHTR MODEL}

A 3-D whole-core exact-geometry model of a VHTR hexagonal-block configuration with a detailed temperature distribution was developed and implemented for calculations with the ORNL SCALE 5.1 code system. The applied VHTR model is shown in Fig. 2 including the prototypic 3D temperature distribution. The fuel assembly blocks are based on the HTTR fuel block configuration. [4,6,21]

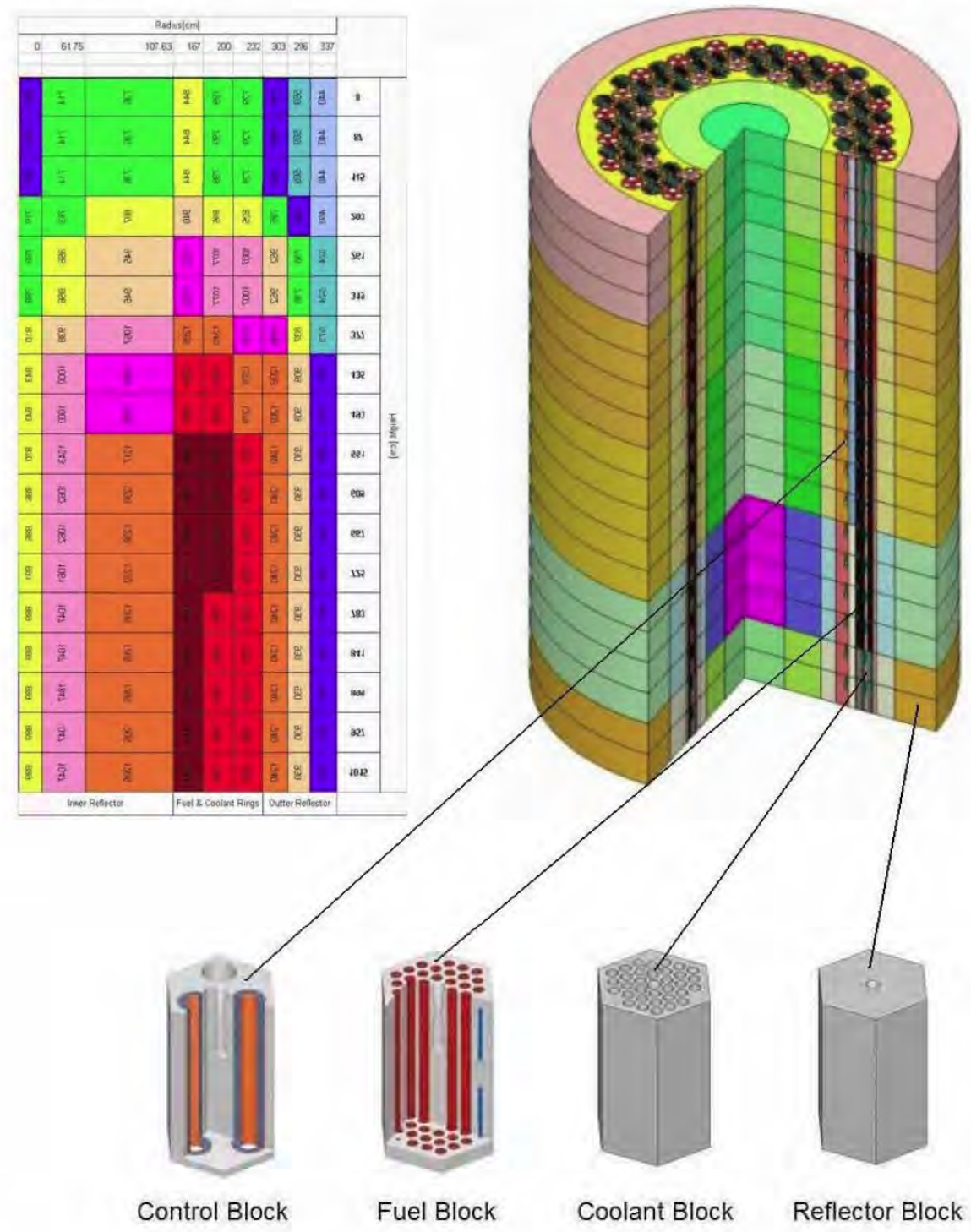

Figure 2. SCALE 5.1 whole-core exact 3D geometry VHTR model with the representative core temperature distribution (KENO3D).

As illustrated in Fig. 2, the developed initial design is consistent with postulated Generation IV VHTR core requirements. [8] To assure consistency with the modeling validation effort, the TRU-fueled VHTR fuel blocks have been designed using the HTTR geometry data. Corresponding parameters of the reference $\mathrm{UO}_{2}$-fueled VHTR configuration and their initial values are given in Table 2. 


\section{Utilization of MAs as a Fuel Component for Ultra-Long Life} VHTR Configurations: Designs, Advantages and Limitations

Table 2. Reference Parameters of the Initial $\mathrm{UO}_{2}$-Fueled VHTR Design

\begin{tabular}{|c|c|c|c|}
\hline Fuel & $\mathrm{UO}_{2}$ & Power $\left(\mathrm{MW}_{\text {th }}\right)$ & 600 \\
\hline Enrichment (\%) & 9 & Power Density $\left(\mathrm{W} / \mathrm{cm}^{3}\right)$ & 6.9 \\
\hline \multirow[t]{2}{*}{ Coolant } & \multirow[t]{2}{*}{$\mathrm{He}$} & Pressure (MPa) & 7.0 \\
\hline & & Inlet/Outlet Temperature $\left({ }^{\circ} \mathrm{C}\right)$ & $490 / 950$ \\
\hline \multirow[t]{3}{*}{ \# of Columns } & \multirow[t]{3}{*}{102} & \# of Fuel Columns & 66 \\
\hline & & \# of Control Columns & 36 \\
\hline & & \# of Blocks/Column & 13 \\
\hline Block Pitch $(\mathrm{cm})$ & 36 & \# of Fuel Pins/Fuel Block & 32 \\
\hline \multirow[t]{7}{*}{ Block Height $(\mathrm{cm})$} & \multirow[t]{7}{*}{58} & \# of $\mathrm{B}_{4} \mathrm{C}$ Rods/Fuel Block & 2 \\
\hline & & Control Rods/Control Block & 2 \\
\hline & & Emergency Rods/Control Block & 1 \\
\hline & & Compact Pitch $(\mathrm{cm})$ & 5.15 \\
\hline & & Fuel Hole Radius (cm) & 4.1 \\
\hline & & Compact Inner Radius (cm) & 0.5 \\
\hline & & Compact Outer Radius $(\mathrm{cm})$ & 1.3 \\
\hline \multirow[t]{7}{*}{ Packing (\%) } & \multirow[t]{7}{*}{30} & $10.41 \mathrm{~g} / \mathrm{cm}^{3}$ Kernel Radius $(\mathrm{cm})$ & 0.02985 \\
\hline & & $1.14 \mathrm{~g} / \mathrm{cm}^{3}$ Buffer Radius $(\mathrm{cm})$ & 0.03588 \\
\hline & & $1.89 \mathrm{~g} / \mathrm{cm}^{3}$ PyCl Radius $(\mathrm{cm})$ & 0.03895 \\
\hline & & $3.20 \mathrm{~g} / \mathrm{cm}^{3}$ SiC Radius $(\mathrm{cm})$ & 0.04184 \\
\hline & & $1.87 \mathrm{~g} / \mathrm{cm}^{3}$ PyC2 Radius $(\mathrm{cm})$ & 0,04645 \\
\hline & & Matrix $\left(\mathrm{g} / \mathrm{cm}^{3}\right)$ & 1.77 \\
\hline & & Block $\left(\mathrm{g} / \mathrm{cm}^{3}\right)$ & 1.69 \\
\hline
\end{tabular}

\section{REACTOR PERFORMANCE CHARACTERISTICS}

A symbiotic quasi-sustainable system consisting of PWRs and TRU-fueled VHTRs can be considered taking advantage of the identified above fuel cycle adaptability. This system cannot be truly sustainable because of fissile feeds from PWRs but it can attain quasi-sustainability for asymptotic configurations where external LEU-feeds of PWRs approach zero.
In the present analysis, potential PWR spent fuel vectors were determined using the IAEA's nuclear fuel cycle simulation system (VISTA code system). To evaluate potential perturbations in TRU vectors and their influence on the VHTR performance characteristics, fuel burnup and load factor values for PWRs were perturbed.

Tolerance to potential variations of fuel loading vectors is especially important for binary systems with significantly varying characteristics of their constituents like PWRs and VHTRs. Figure 3 illustrates the observed sensitivity of PWR TRUs with respect to the reactor operation conditions and parameters. The reference TRU vector was determined from the analysis of legacy spent fuel vectors of PWRs. [7]

The use of coated micro-particles (see Fig. 1) creates double-heterogeneity that defines performance characteristics of the VHTR core. The first level of heterogeneity (microlevel) is formed by coated fuel micro-particles that are randomly distributed in the graphite compact. The second level of heterogeneity (macro-level) is formed by the prismatic blocks with compacts that compose the VHTR core. For desirable performance characteristics, the prismatic graphite block core has channels for coolant, instrumentation and handling operations.

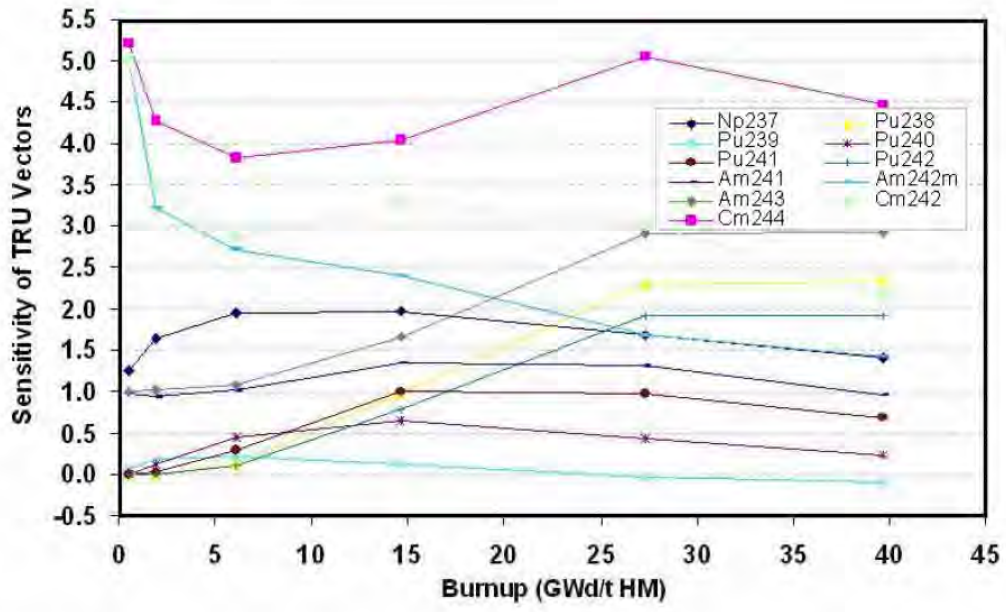

Figure 3. Fluctuations of PWR TRU vectors due to variations of reactor operation characteristics.

As a result, there are various neutron streaming passages formed by voids, coolant channels, instrumentation and handling channels, plenums, and etc. (see Fig. 2) The proximity to outer and central reflector regions is also an important factor that influences the VHTR physics. Depending on a specific computational model, these features act and can be taken into account as multiple additional levels of heterogeneity up to the $3 \mathrm{D}$ finite core model that provides an appropriate 


\title{
Utilization of MAs as a Fuel Component for Ultra-Long Life VHTR Configurations: Designs, Advantages and Limitations
}

\begin{abstract}
comprehensive multi-heterogeneity treatment (see Fig. 2). This principal arrangement creates phenomena complicating analysis of these systems. It is apparent that the doubleheterogeneity effects should be expected to play a defining role for all nuclides in the VHTR cores, especially those with

${ }^{238} \mathrm{U}$ and burnable absorbers like erbium. Furthermore, as compared to the exact geometry modeling using Monte Carlo codes, the simplified single-level macro-heterogeneity modeling could significantly over estimate fuel lifetimes in VHTRs. [16]
\end{abstract} resonance cross sections, including fuel nuclides like ${ }^{235} \mathrm{U}$ and

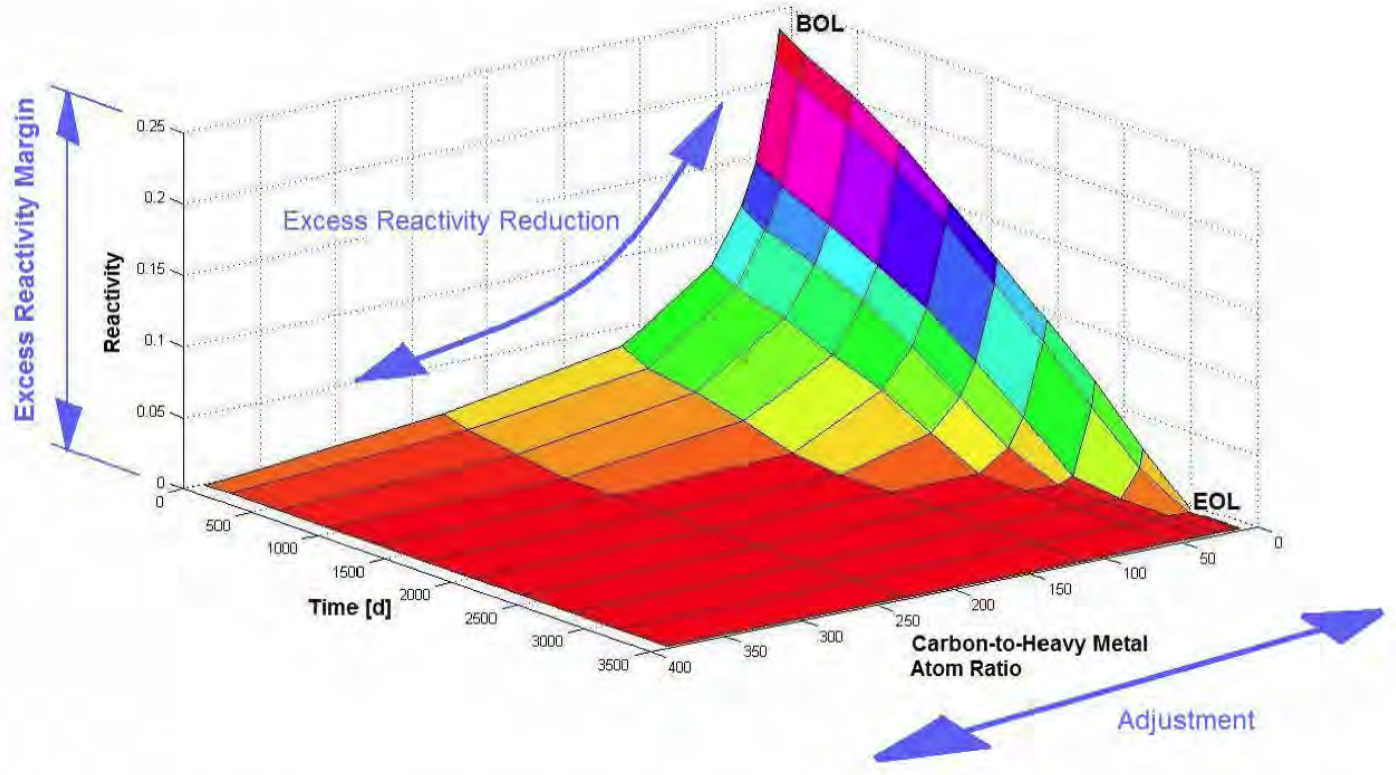

Figure 4. Reductions of initial excess reactivity levels in the LEU-fueled VHTR configurations as a function of the carbon-to-heavy metal atom ratio values.

At the same time, it gives flexibility in fuel selection, management, core performance and adaptability to the changing economics. The same reactivity variations can be attained as a result of different changes of the basic VHTR core parameters. The LEU- and TRU-fueled VHTR prismatic core configurations have inherent flexibility in the component configuration allowing, for example, for the carbon-to-heavy metal $(\mathrm{C} / \mathrm{HM})$ atom ratio to be adjusted as a result of changes in fuel compact dimensions, hexagonal block parameters, or changes in numbers of TRISO particles per compact. Figure 4 shows that these adjustments allow achieving substantial reductions in initial excess reactivity levels.

According to Fig. 5, the $\mathrm{C} / \mathrm{HM}$ adjustments shift neutron spectra towards higher energies. Harder spectra, in the case of TRU fuels, result in self-stabilization effects extending operation without intermediate refueling.

Up to nine years of continuous operation were found to be possible for the single-batch TRU-fueled VHTR configurations without intermediate refueling. Four year core-lifetimes were found to be possible for the single-batch LEU-fueled VHTR configurations without intermediate refueling. Figure 6 illustrates advantages of the TRU-fueled VHTR configurations for OTTO modes of continuous operation. The longevity of the core is limited by attainable fast fluence levels as permitted by material selections. $[13,14]$

\section{POWER UNIT PERFORMANCE CHARACTERISTICS}

Further reductions in initial reactivity levels can be achieved by deploying burnable poisons in the core. $[22,23]$ As illustrated in Fig. 7, burnable poisons also offer potential minimization of operational reactivity swings in the VHTR configurations simplifying system control options.

Preliminary evaluations show that the VHTR power unit configurations can have sufficiently high energy conversion efficiencies up to $57 \%$ even for simple Brayton cycles that allow for multiple simultaneous applications of produced high temperature heat assuming operating temperatures around $800^{\circ} \mathrm{C}$. 
Utilization of MAs as a Fuel Component for Ultra-Long Life VHTR Configurations: Designs, Advantages and Limitations

\section{CONCLUSIONS}

As presented in this paper, the studies of TRU-fueled VHTR suggest potentially promising performance characteristics of VHTRs as autonomous systems for cogeneration applications. The primary purpose of added TRUs is to achieve prolonged operation on a single fuel loading. Researchers assessed perturbations of the VHTR spent fuel characteristics (back-end management options) because of the VHTR fresh fuel composition deviations from the chosen reference content. A single-batch autonomous operation (no onsite refueling) offers a number of advantages facilitating deployment in energy-demanding developing regions with limited industrial infrastructure while maintaining system safety and security by design.

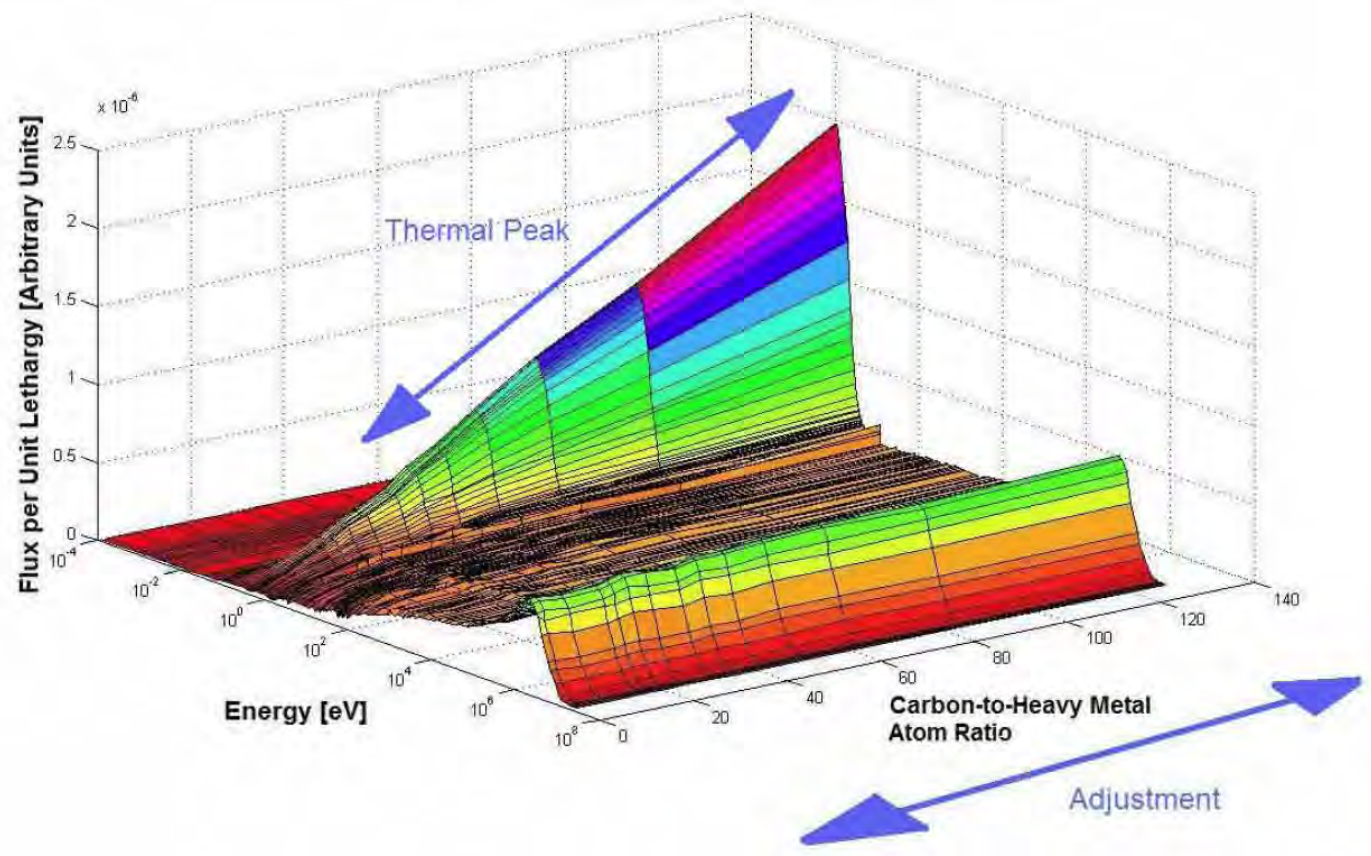

Figure 5. Spectral variations in the TRU-fueled VHTR configurations as a function of the carbon-to-heavy metal atom ratio values.

Substantial differences between the traditional LEU- and advanced TRU-fueled VHTR configurations have been observed. The use of TRU-vectors in VHTRs leads to prolonged single-loading OTTO modes. Up to nine years of continuous operation were found to be possible for the singlebatch TRU-fueled VHTR configurations without intermediate refueling. Four year core-lifetimes were found to be possible for the single-batch LEU-fueled VHTR configurations without intermediate refueling. The corresponding destruction rates are about $30 \%$. At the same time, radiotoxicity levels in nuclear waste streams require certain cooling times to drop below initial levels.

The viable longevity of the VHTR configurations is limited by attained fast fluence levels. This limitation can be reduced or eliminated using radiation-tolerant materials. The VHTR configurations have sufficiently high energy conversion efficiencies even for simple Brayton cycles that allow for multiple simultaneous applications of produced high temperature heat. Essentially, the entire spectrum of cogeneration technologies can be implemented in the "all-in-one" VHTR configuration. However, further safety studies are required to confirm acceptable dynamic response of the considered VHTR systems.

The research effort enhances capabilities of the Generation IV VHTR and potentially transforms it to a technology that can deliver electricity, hydrogen, potable water and industrial heat as well as assist in spent nuclear fuel management. 


\section{S \\ Research Initiative}

Utilization of MAs as a Fuel Component for Ultra-Long Life VHTR Configurations: Designs, Advantages and Limitations

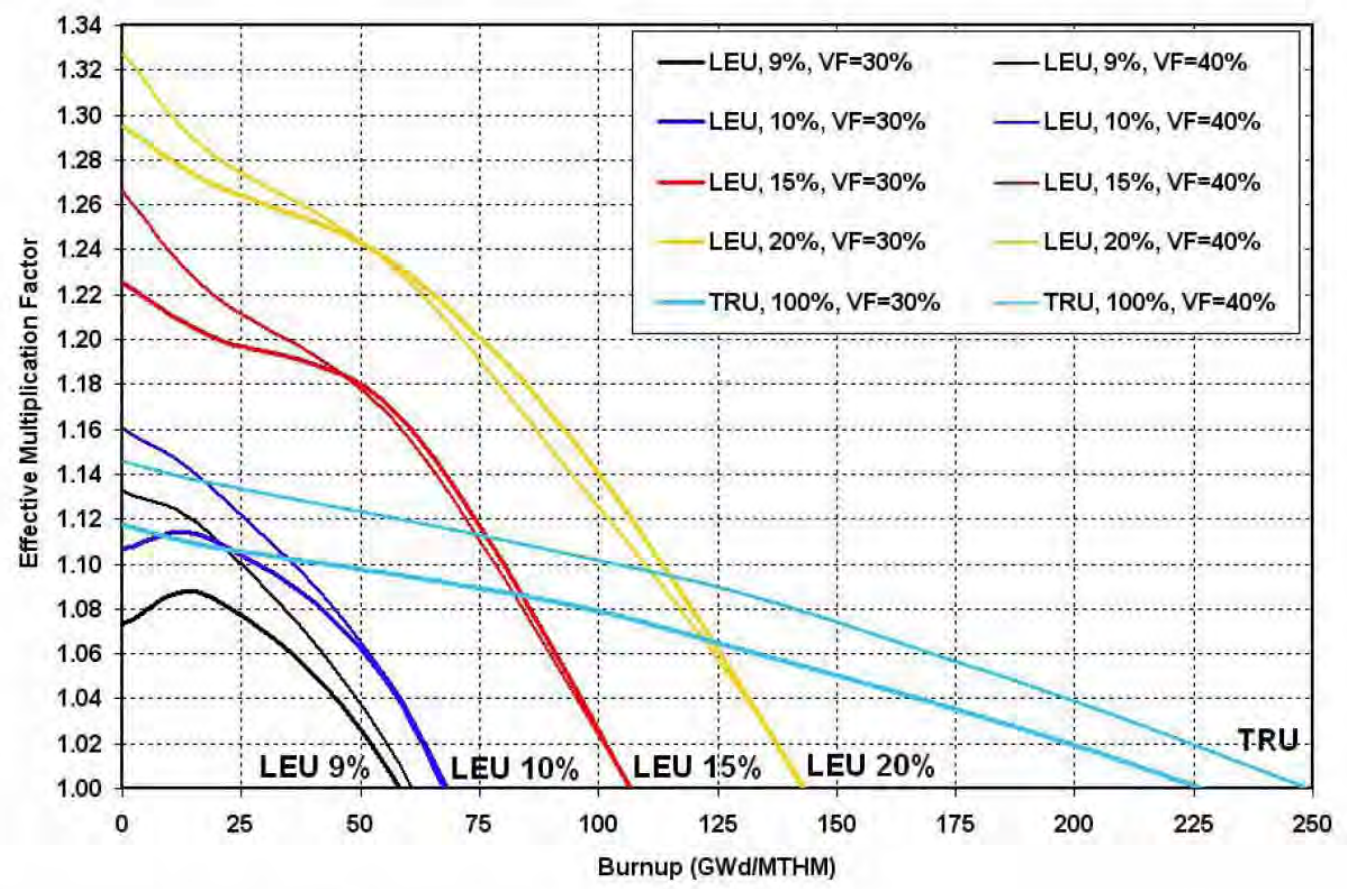

Figure 6. OTTO modes of LEU- and TRU-fueled VHTR configurations.

\section{ACKNOWLEDGMENTS}

This paper is based upon work supported by the U.S. Department of Energy under Award Number DE-FC0705ID14655 (05-094).

\section{DISCLAIMER}

This paper was prepared as an account of work sponsored by an agency of the United States Government. Neither the United States Government nor any agency thereof, nor any of their employees, makes any warranty, express or implied, or assumes any legal liability or responsibility for the accuracy, completeness, or usefulness of any information, apparatus, product, or process disclosed, or represents that its use would not infringe privately owned rights.

Reference herein to any specific commercial product, process, or service by trade name, trademark, manufacturer, or otherwise does not necessarily constitute or imply its endorsement, recommendation, or favoring by the United States Government or any agency thereof.

The views and opinions of authors expressed herein do not necessarily state or reflect those of the United States Government or any agency thereof. 


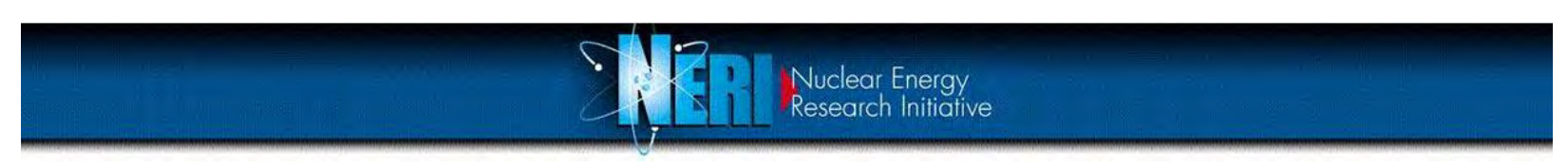

\section{Utilization of MAs as a Fuel Component for Ultra-Long Life VHTR Configurations: Designs, Advantages and Limitations}

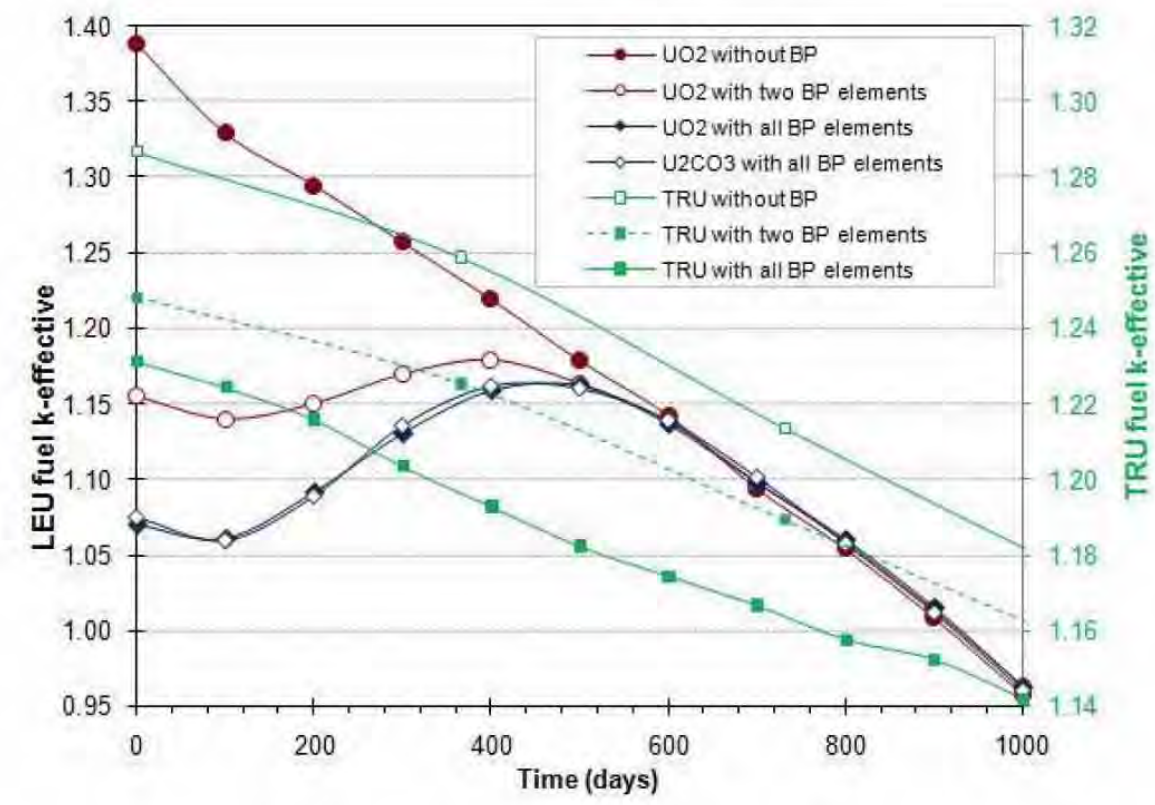

Figure 7. Burnable poison effects on the VHTR configurations operating in the OTTO mode.

\section{REFERENCES}

1. A. B. ALAJO, P. V. TSVETKOV, "Reactor Physics of VHTRs with $\mathrm{UO}_{2}, \mathrm{UCO}$ and $\mathrm{UC}_{0.5} \mathrm{O}_{1.5}$ Kernels", Trans. Amer. Nucl. Soc. and Embedded Topical Meetings on Isotopes for Medicine and Industry and Nuclear Fuels and Structural Materials for the Next Generation Nuclear Reactors, 98, 736-737 (2008).

2. D. E. AMES II, "Configuration Adjustment Potential of the VHTR Prismatic Cores with Advanced Actinide Fuels", Thesis, Master of Science, August 2006, Nuclear Engineering, Texas A\&M University (2006).

3. G. B. BRUNA et al., "Uncertainty Analysis and Optimization Studies on the Deep-Burner-Modular Helium Reactor (DB-MHR) for Actinide Incineration", Proc. PHYSOR2004 - The Physics of Fuel Cycle and Advanced Nuclear System: Global Developments, Chicago, USA, April 25-29, 2004, American Nuclear Society, Lagrange Park, IL. (2004).

4. "Critical Experiments and Reactor Physics Calculations for Low-Enriched HTGRs", IAEA-TECDOC-1249, International Atomic Energy Agency, Vienna, Austria (2001).

5. M. EHRGOTT, Multicriteria Optimization, Springer Verlag, Berlin (2000).

6. "Evaluation of High Temperature Gas-cooled Reactor Performance: Benchmark Analysis Related to Initial
Testing of the HTTR and HTR-10", IAEA-TECDOC1382, International Atomic Energy Agency, Vienna, Austria (2003)

7. "Final Environmental Impact Statement for a Geologic Repository for the Disposal of Spent Nuclear Fuel and High-Level Radioactive Waste at Yucca Mountain, Nye County, Nevada", Vol. 2, Appendix A, "Inventory and Characteristics of Spent Nuclear Fuel, High-Level Radioactive Waste, and Other Materials", DOE/EIS-0250, U.S. DOE (2002).

8. "Generation IV Nuclear Energy Systems: Ten Year Program Plan", March 2005, Office of Advanced Nuclear Research, DOE Office of Nuclear Energy, Science, and Technology, U.S. DOE (2005).

9. D. F, JONES, S. K. MIRRAZAVI, M. TAMIZ, "Multiobjective Metaheuristics: An Overview of the Current State-of-the-Art", European Journal of Operational Research, 137(1), 1-9 (2002).

10. T. KIM, T. TAIWO, R. HILL, W. YANG, F, VENNERI, "A Feasibility Study of Reactor-Based Deep-Burn Concepts", ANL-AFCI-155, Argonne National Laboratory (2005).

11. K. KUNITOMI, "Development of New Type of HTGR", Proc. 73rd JSME Fall Annual Meeting, JSME, Japan, 1995.

12. T. LEWIS, D. AMES, P. TSVETKOV, "Analysis of TRUFueled VHTR Prismatic Core Performance Domains", Trans. Amer: Soc., 97, 859 (2007),

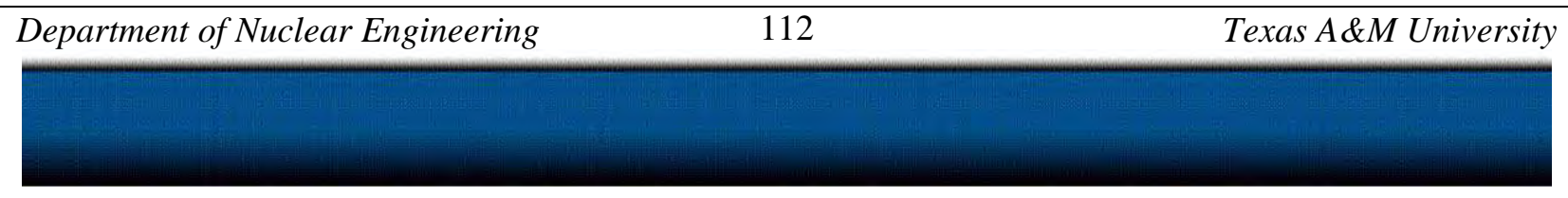




\section{Utilization of MAs as a Fuel Component for Ultra-Long Life VHTR Configurations: Designs, Advantages and Limitations}

\section{Project 05-094}

13. K. MINATO, T. OGAWA, K. SAWA, A. ISHIKAWA, T. TOMITA, S. IIDA, H. SEKINO, "Irradiation Experiment on ZrC-Coated Fuel Particles for High Temperature GasCooled Reactors", Nucl. Techn., 130, 272 (2000).

14. K. MINATO, K. SAWA, T. KOYA, T. TOMITA, A. ISHIKAWA, "Fission Product Release Behavior of Individual Coated Fuel Particles for High Temperature Gas-Cooled Reactors", Nucl. Techn., 131, 36 (2000).

15. C. M. N. A. PEREIRA, "Evolutionary Multicriteria Optimization in Core Design: Basic Investigations and Case Study", Annals of Nuclear Energy, 31, 1251-1264 (2004).

16. R. PLUKIENE, D. RIDIKAS, "Modeling of HTRs with Monte Carlo: from a Homogeneous to an Exact Heterogeneous Core with Microparticles", An. Nucl. En., 30, 1573, (2003).

17. "Report to Congress on Advanced Fuel Cycle Initiative: The Future Path for Advanced Spent Fuel Treatment and Transmutation Research", 03-GA50439-06, January, 2003, Office of Nuclear Energy, Science, and Technology, U.S. DOE (2003).

18. M.B. RICHARDS et.al. " $\mathrm{H}_{2}-\mathrm{MHR}$ Pre-conceptual Design Report: SI-Based Plant," GA-A25401, General Atomics, San Diego, USA (2006).

19. C. RODRIGUEZ, A. BAXTER, D. MCEACHERN, M. FIKANI, F. VENNERI, "Deep-Burn: Making Nuclear Waste Transmutation Practical", Nucl. Eng. Design, 2805(1) (2003).

20. "SCALE: A Modular Code System for Performing Standardized Computer Analyses for Licensing Evaluation", ORNL/TM-2005/39,Ver.5.1, November 3, 2006, Oak Ridge National Laboratory, Tennessee (2006).

21. T. A. TAIWO, T. K. KIM, W. S. YANG, H. S. KHALIL, "Evaluation of High Temperature Gas-Cooled Reactor Physics Experiments as VHTR Benchmark Problems", ANL-GenIV-059, Argonne National Laboratory, September 2005.

22. A. TALAMO, "Conceptual Design of QUADRISO Particles for High Temperature Reactors", Trans. Amer: Nucl. Soc. and Embedded Topical Meetings on Isotopes for Medicine and Industry and Nuclear Fuels and Structural Materials for the Next Generation Nuclear Reactors, 98 , 727-728 (2008).

23. H. N. TRAN, Y. KATO, "An Optimal Loading Principle of Burnable Poisons for an OTTO Refueling Scheme in Pebble Bed HTGR Cores", Proc. 2008 Intern. Congress on Advances in Nucl. Power Plants, Embedded Topical Meeting, June 8-12, 2008, Anaheim, California, USA, 289297 (2008).

24. P. TSVETKOV, A. ALAJO, D. AMES, M. PRITCHARD, "Spectrum Shifting as a Mechanism to Improve Performance of VHTRs with Advanced Actinide Fuels", Proc. 14th Intern. Conf. Nucl. Eng. (ICONE 14), Miami, Florida, July 17 -20, 2006, ASME (2006).

\section{Final Scientific/Technical Report}

25. P. V. TSVETKOV, D. E. AMES II, M. L. PRITCHARD, A. B. ALAJO, T. G. LEWIS III, "TRU-Fueled VHTRs: Design, Performance, and Applications", Proc. GLOBAL2007: Advanced Fuel Cycles and Systems, September 9-13, 2007, Boise, Idaho, 852-855, USA, (2007).

26. P. V. TSVETKOV, T. G. LEWIS III, A. B. ALAJO, "TRUFueled VHTRs for Applications Requiring an Extended Operation with Minimized Control and No Refueling", Proc. $16^{\text {th }}$ Intern. Conf. Nucl. Eng. (ICONE 16), Orlando, Florida, May 11-15, 2008, ASME (2008).

27. P. V. TSVETKOV, T. G. LEWIS III, A. B. ALAJO, "Utilization of TRUs in VHTRs - Operation in a SingleBatch Mode: Front End, Back End, and Performance", Trans. Amer. Nucl. Soc. and Embedded Topical Meetings on Isotopes for Medicine and Industry and Nuclear Fuels and Structural Materials for the Next Generation Nuclear Reactors, 98, 733-735 (2008).

28. P. V. TSVETKOV, A. B. ALAJO, T. G. LEWIS III, D. E. AMES II, "Out-of-Core Fuel Cycle Characteristics of VHTRs with No On-Site Refueling", Proc. 2008 Intern. Congress on Advances in Nucl. Power Plants, Embedded Topical Meeting, June 8-12, 2008, Anaheim, California, USA, 298-302 (2008) 


\title{
6.10. Autonomous Control Strategies for VHTR-Based Systems for Hydrogen Production
}

\author{
Pavel V. Tsvetkov, Ayodeji B. Alajo, David E. Ames II \\ Department of Nuclear Engineering \\ Texas A\&M University \\ 129 Zachry Engineering Center, MS 3133 TAMU, College Station, Texas 77843-3133, USA \\ Phone: 979/845-7078, \\ Fax: 979/845-6443 \\ E-mail:Tsvetkov@tamu.edu
}

\begin{abstract}
This paper is focused on feasible autonomous control strategies for Generation IV Very High Temperature Reactors (VHTR)-based systems for hydrogen production. Various burnable poison distributions and fuel compositions are considered. In particular, utilization of TRUs in VHTRs is explored as the core self-stabilization approach. Both direct cycle and indirect cycle energy conversion approaches are discussed. It is assumed that small-scale VHTRs may be considered for international deployment as grid-appropriate variable-scale self-contained systems addressing emerging demands for hydrogen. A Monte Carlo-deterministic analysis methodology has been implemented for coupled design studies of VHTRs with TRUs using the ORNL SCALE 5.1 code system. The developed modeling approach provides an exactgeometry 3D representation of the VHTR core details properly capturing VHTR physics. The discussed studies are being performed within the scope of the U.S. DOE NERI project on utilization of higher actinides (TRUs and partitioned MAs) as a fuel component for extended-life VHTR configurations.
\end{abstract}

\begin{tabular}{|c|c|}
\hline \multicolumn{2}{|c|}{ NOMENCLATURE } \\
\hline HTGR & - High Temperature Gas-cooled Reactor \\
\hline HTTR & High Temperature Test Reactor \\
\hline LEU & Low-Enriched Uranium \\
\hline MA & - Minor Actinides \\
\hline MO & - Multi-objective Optimization \\
\hline NERI & - Nuclear Energy Research Initiative \\
\hline NGNP & - Next Generation Nuclear Plant \\
\hline OTTO & - Once-Through-Then-Out \\
\hline TRU & - Transuranium Nuclides \\
\hline VHTR & - Very High Temperature Reactor \\
\hline
\end{tabular}

\section{INTRODUCTION}

This paper is focused on feasible autonomous control strategies for Generation IV Very High Temperature Reactors (VHTR)-based systems for hydrogen production. Various burnable poison distributions and fuel compositions are considered. In particular, utilization of TRUs in VHTRs is explored as the core self-stabilization approach. The resulting configurations should also have an inherent potential for autonomous operation with minimized maintenance. It is expected that LWRs will eventually be replaced by Generation IV VHTRs. At that time, sustainable nuclear systems consisting of FRs and VHTRs will become possible. Both direct cycle and indirect cycle energy conversion approaches are discussed. It is assumed that small-scale VHTRs may be considered for international deployment as grid-appropriate variable-scale self-contained systems addressing emerging demands for hydrogen

Behavior of the advanced nuclear energy systems like Generation IV VHTRs is difficult to predict due to inherent safety features resulting in tight coupling between energy production, transport and conversion, and system behavior during normal operation and off-normal situations. [14,15] The systems should have satisfactory performance characteristics in several inherently noncommensurable areas simultaneously. [2]

A Monte Carlo-deterministic analysis methodology has been implemented for coupled design studies of VHTRs with TRUs using the ORNL SCALE 5.1 code system. [16] The developed modeling approach provides an exact-geometry $3 \mathrm{D}$ representation of the VHTR core details properly capturing VHTR physics. Searches for acceptable performance domains are handled as trade-off optimization problems with multidisciplinary design criteria including output requirements,

\begin{tabular}{lll}
\hline Department of Nuclear Engineering & 114 & Texas A\&M University \\
\hline &
\end{tabular}




\section{Utilization of MAs as a Fuel Component for Ultra-Long Life} VHTR Configurations: Designs, Advantages and Limitations

\section{Project 05-094}

Final Scientific/Technical Report safety characteristics, system longevity, waste minimization. envirommental impacts, economics, and other characteristics.

Evidently, there is no a unique system configuration that equally meets all criteria at the same time. Only equally acceptable (preference non-dominated) end-configurations can be found. Such configurations form a set of Pareto-optimal solutions representing a compromise between postulated design criteria. Promising multi-objective optimization (MO) strategies can be developed using stochastic search mechanisms of sufficiently robust genetic algorithms (GA) that allow surveying the entire space of performance characteristics. Existing sensitivities and uncertainties in input parameters and performance characteristics are taken into account.

The discussed studies are being performed within the scope of the U.S. DOE NERI project on utilization of higher actinides (TRUs and partitioned MAs) as a fuel component for extended-life VHTR configurations. Based on the initial evaluations of the VHTR with TRUs/MAs, the major thrust is the detailed analysis of configuration variation capabilities to achieve prolonged operation without refueling, maximize burnup, and minimize reactivity swings. [5,20,22,23]

The TRU-fueled VHTR systems are developed and analyzed accounting for controllability, dynamics and safety margins during the entire reactor lifetime. The resulting configurations should have inherent capabilities for utilization of TRUs from spent LWR fuel. [5]

\section{VHTR CORE MODEL}

To create advanced nuclear energy systems it is desirable to have a high fidelity modeling-based design development that relies on simulating features of the entire life cycle of the system before actual physical prototyping - from concept development to detailed design, prototyping, and safety analysis. This approach would provide consistency between neutronics and other performance aspects such as thermal hydraulics, mechanical integrity, plant heat transfer and conversion balances. It is convenient to consider both. requirements and constraints, as objectives with special characteristics. The multi-objective optimization process can be developed as shown in Fig. 1. The framework requires solving specialized multi-level non-convex optimization problems with large numbers of variables. These problems involve objectives with certain hierarchical structure imposed by the specifics of postulated constraints. $[8,12,21]$

The design development begins with the inception of a system concept. The system concept helps defining objectives. The representative stochastic model with assumed uncertainty distributions for model parameters initiates the optimization process. Postulated uncertainty distributions at the model parameterization stage lead to the corresponding uncertainties in system performance characteristics. Ultimately, once Pareto optimality criteria are met under the defined set of objectives. the end-system configuration can be finalized. Because solving multi-objective optimization problems is a very difficult task. in addition to Pareto-based techniques, multi-objective evolutionary algorithms can be applied. Some of the vector evaluated genetic algorithms could also facilitate the solution process. There are no universal optimization techniques, the optimization step should be an intelligent combination of several algorithms like Non-dominated Sorting Genetic Algorithm, Niched-Pareto Genetic Algorithm, Multi-Objective Genetic Algorithm, Strength Pareto Evolutionary Algorithm. Pareto Archived Evolution Strategy, Non-dominated Sorting Genetic Algorithm. [12]

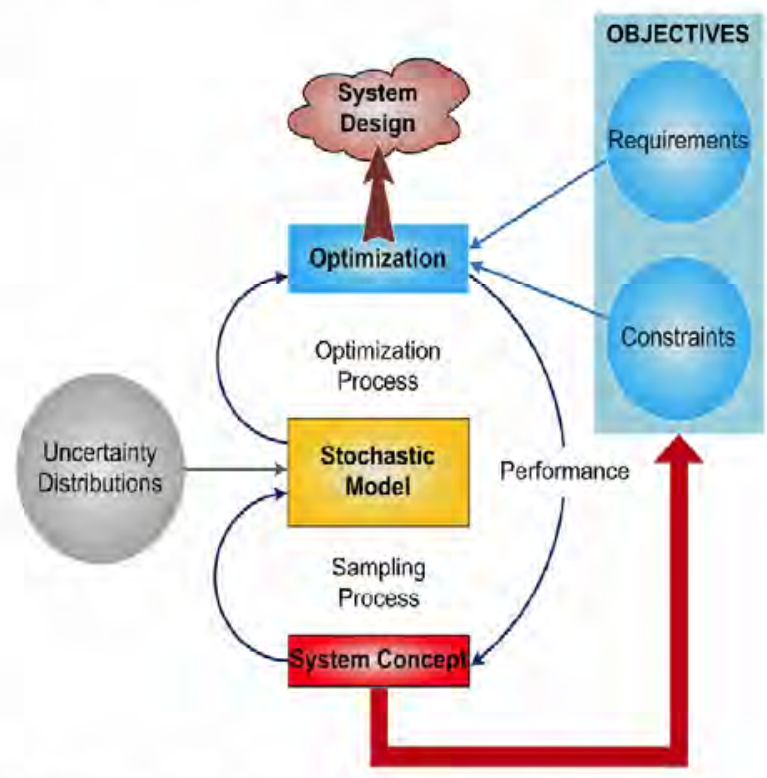

Figure 1. Design development and optimization.

The suggested integrated/hybrid Monte-Carlodeterministic modeling approach offers the desirable analysis capabilities for the VHTR design studies. The use of Monte Carlo codes creates a potential for explicit 3D wholecore/reactor studies of the VHTR configurations addressing the key reactor physics areas such as the double and multi-level heterogeneity, neutron streaming in the low-density regions. and neutron spectrum transitions at the interfaces. [13] The 3D whole-core exact-geometry VHTR model has been developed for use in calculations with SCALE 5.1 code system. The model takes into account TRISO micro-particles, fuel compacts, fuel assembly blocks, control rod blocks, replaceable reflectors and a permanent outer reflector. The project uses the actual test results and benchmark problems of the HTTR Program (prismatic core design, reactor experiments). [1,22]

The VHTR model is a nearly explicit representation of the existing HTTR core configuration. The model was created in the SCALE code system utilizing the CSAS6/KENO-VI module. Obtained benchmark results are in agreement with the

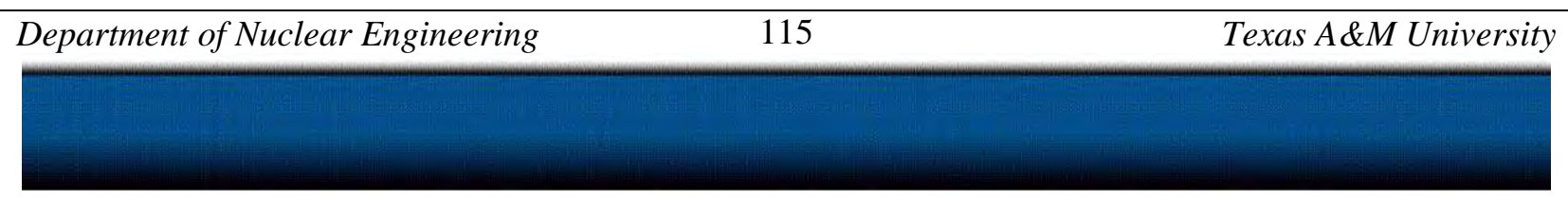




\section{Utilization of MAs as a Fuel Component for Ultra-Long Life VHTR Configurations: Designs, Advantages and Limitations}

avidiable HTTR data and confirm applicability of the chosen modeling melhodology. Each of the benchmark cases is within $0.25 \%$ of the experimental values and fall within experimental erros. [1]

As shown in Fig. 2, the basis configuration has been developed using the Generation IV VHTR core specifications and the HITR hexagonal block geometry data: $[4,6]$ The HTTR is eurrently the only operating VHTR prismatic core design, making it a focal point of VHTR related research. [3.4.17]
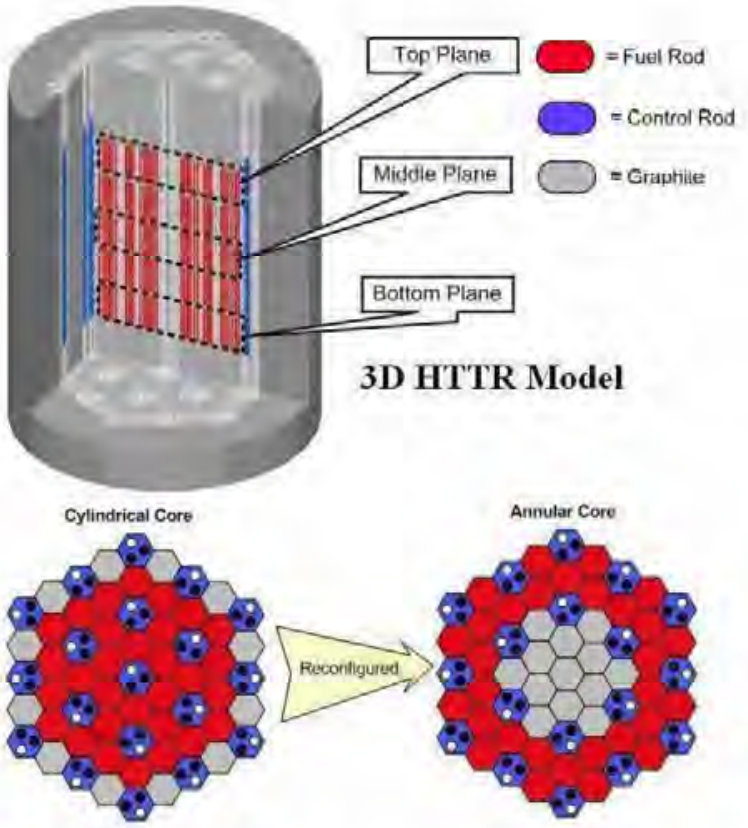

Volume $=75.8 \mathrm{~m}^{3}$

Total Fuel Assembly Blocks $=150$

Core height $=522 \mathrm{~cm}$

3D Small-Scale Reactor Mode

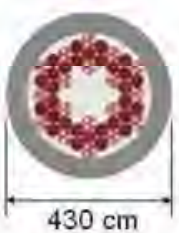

$430 \mathrm{~cm}$

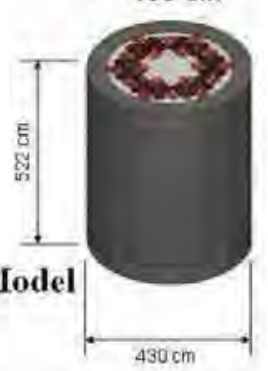

Figure 2. Small-scale VHTR configuration (HTIR basis).
The prismatic whole-core 3D model was adjusted from the original cylindrical core of the HTTR to that of an ammular core. The annular core is one of the promising core types for the future VHTRs because of its high imherent safery characteristics following a loss of coolant accident. The decay heat removal is enhanced by introduction of the annular core because the heat transfer path will be shortened due to the relatively thin active core region. As a result, the fuel temperature in a loss of coolant acciolent can be maintamed at less than the finel temperature limit of $1600^{\circ} \mathrm{C}$.

The transition from the HTTR basis to the small-scale VHTR configuration included changing the fitel in the core from a mixture of uranium entichments ( 12 types) to just one enrichment throughout the entire core $(8 \mathrm{wt} \%)$. To create the annular core the model was reconfigured by replacing the fuel assembly block columms located in the inner region of the core with the replaceable reflector block columns in the outer region, and vice versa. An exact representation of the configuration adjustment is provided in Fig. 2.

\section{SAFETY \& CONTROL OF SMALL-SCALE VHTR-H}

The VHTR systems achieve their safety through their design approach, the materials used, and the fuel form. The key safety features of VHTRs, in addition to the radionuclide retention capability of the TRISO fuel particle, is a small operational excess reactivity, a large negative temperature coefficient, and a passive heat removal capability of the reactor design. The combination of the small excess reactivity and large negative temperature coefficient stops the nuclear fission process with only a moderate temperature rise in the core even if the confrol and shutdown systems fail.

In addition, the introduction of an annular core allows fuel decay heat to be conducted through the reactor structures to the vessel cavity and then to the atmosphere without outside intervention. This provides the reactor with a high degree of inherent safety, in turn, making the temperature coefficient a major component for safety analysis of VHTRs.

Table I shows the calculated values obtained for the isothermal temperature coefficients for the HTTR basis and the corresponding cylindrical and annular configurations of the small-scale VHTR The isothermal temperature coefficients for each core configuration are within the standard deviation limits of one another.

Table 1. Isothermal Temperature Coefficients of SmallScale VHTR Configurations

\begin{tabular}{|c|c|}
\hline VHTR Configuration & $\begin{array}{l}\text { Isothermal Temperature Coefficient, } 10^{-6} \\
\left(1 /{ }^{\circ} \mathrm{C}\right)\end{array}$ \\
\hline HTTR & $-9.626 \div 2.409$ \\
\hline Cylindrical Core & $-6.670=1.415$ \\
\hline Anmular Core & $-7.817=1.474$ \\
\hline
\end{tabular}

Table 2 summarizes basic reactor physics characteristics obtained for the fuel loadings in the (a) cylindrical core configuration and in the (b) annular core configuration. The 


\section{Utilization of MAs as a Fuel Component for Ultra-Long Life} VHTR Configurations: Designs, Advantages and Limitations

\section{Project 05-094}

Final Scientific/Technical Report

VHTR configurations include conventional fuels like $\mathrm{UO}_{2}$ as well as advanced fuels with compositions containing UC, MOX, and/or TRUs.

Table 2. Basic Reactor Physics of the Small-Scale VHTR Cylindrical and Annular Core Configurations

(a) Cylindrical Core Configuration

\begin{tabular}{|c|c|c|c|c|}
\hline Fuel & $k_{\text {eff }}$ & $\begin{array}{l}\text { Fission-Inducing } \\
\text { Energy (eV) }\end{array}$ & $\begin{array}{l}\text { System Mean } \\
\text { Free Path (cm) }\end{array}$ & $\begin{array}{l}\text { Fission Neutron } \\
\text { Yield }\end{array}$ \\
\hline $\begin{array}{l}\mathrm{UO}_{2}, \\
8 \%\end{array}$ & $\begin{array}{r}1.099 \\
\quad 00.001\end{array}$ & ]$^{0,2504}$ & $4^{2.6897}=0.0006$ & $6^{2.43859} \pm 0.00001$ \\
\hline $\begin{array}{l}\text { UC, } \\
8 \%\end{array}$ & $\begin{array}{r}1.110 \\
=0.001\end{array}$ & $1^{0.2639}$ & $4^{2.6914}=0.0006$ & $6^{2.43871}=0.00001$ \\
\hline TRU & $\begin{aligned} 1.014 & =0.001\end{aligned}$ & $1^{9.43}$ & $5^{2.7218}=0.0007$ & $7^{2.90387}=0.00004$ \\
\hline RGPu & $\underbrace{1.205} \pm 0.001$ & 19.32 & $a^{2.7250} \pm 0.0008$ & $\left.\mathrm{~s}\right|^{2.90006} \pm 0.00001$ \\
\hline
\end{tabular}

(b) Amular Core Configuration

\begin{tabular}{|c|c|c|c|c|}
\hline Fuel & $k_{\text {eff }}$ & $\begin{array}{l}\text { Fission-Inducing } \\
\text { Energy (eV) }\end{array}$ & $\begin{array}{l}\text { System Mean } \\
\text { Free Path (cm) }\end{array}$ & $\begin{array}{l}\text { Fission Neutron } \\
\text { Yield }\end{array}$ \\
\hline $\begin{array}{l}\mathrm{UO}_{2}, \\
8 \%\end{array}$ & $\begin{aligned} 1.078 & \\
& =0.001\end{aligned}$ & $=0.0004$ & $\begin{array}{c}2.6409 \\
4=0.0004 \\
\end{array}$ & ${ }^{2.43861} \pm 0.00001$ \\
\hline $\begin{array}{l}\text { UC, } \\
8 \%\end{array}$ & $\begin{array}{r}1.090 \\
=0.002\end{array}$ & \pm 0.0004 & $\begin{array}{l}2.6424 \\
\pm 0.0005\end{array}$ & $\begin{array}{r}2.43870 \\
=0.00001\end{array}$ \\
\hline TRU & $\begin{aligned} 1.015 & =0.001\end{aligned}$ & $\neq 0.04$ & $\begin{array}{l}2.6669 \\
4=0.0006\end{array}$ & $\stackrel{2.90356}{=}=0.00004$ \\
\hline RGPu & $\begin{array}{r}1.196 \\
=0.001\end{array}$ & 7.32 & $\begin{array}{l}2.6668 \\
\pm 0.0006\end{array}$ & 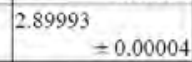 \\
\hline
\end{tabular}

The corresponding initial excess reactivity values and the isothermal temperature coefficients for each fuel loading are given in Table 3. The coefficients in all cases are negative. Achievability of small initial excess reactivity values suggests potential reduction options for control system requirements assuming that desirable configuration longevities are attainable.

Table 3. Initial Excess Reactivities and Isothermal Temperature Coefficients of the Small-Scale VHIR Cylindrical and Annular Core Configurations

\begin{tabular}{|c|c|c|}
\hline Fuel & $\begin{array}{l}\text { Initial Excess } \\
\text { Reactivity (\%) }\end{array}$ & $\begin{array}{l}\text { Isothermal Temperature Coefficient, } 10^{-5} \\
\left(1^{-5} \mathrm{C}\right)\end{array}$ \\
\hline $\begin{array}{l}\mathrm{UO}_{2}, \\
8 \%\end{array}$ & 9,90 & $-6.670 \pm 1.415$ \\
\hline $\begin{array}{l}\text { UC, } \\
8 \%\end{array}$ & 11.0 & $-7.699=1.508$ \\
\hline TRU & 1.40 & $-7.097 \pm 1.148$ \\
\hline RGPu & 20.5 & $-3.526 \pm 0.978$ \\
\hline
\end{tabular}

(b) Annular Core Configuration

\begin{tabular}{|l|c|c|}
\hline Fuel & $\begin{array}{l}\text { Initial Excess } \\
\text { Reactivity }\end{array}$ & $\begin{array}{l}\text { Isothermal Temperature Coefficient, } 10^{5} \\
\left(1 /{ }^{\circ} \mathrm{C}\right)\end{array}$ \\
\hline $\mathrm{UO}_{3}$. & 7.80 & $-7.817 \pm 1.474$ \\
\hline $8 \%$ & & $-6.445 \pm 1.627$ \\
\hline $\mathrm{UC}$, & 9.00 & $-5.085 \pm 1.228$ \\
\hline $8 \%$ & 1.50 & $-3.279 \pm 1.080$ \\
\hline $\mathrm{TRU}$ & 19.6 & \\
\hline $\mathrm{RGPU}$ & & \\
\hline
\end{tabular}

The VHTR power unit configurations can have sufficiently high energy conversion efficiencies up to $57 \%$ even for simple Brayton cycles that allow for multiple simultaneous applications of produced high temperature heat assuming operating temperatures around $800^{\circ} \mathrm{C}$. Consequently, accounting for $\mathrm{H}_{2}$-production components, control options include flow/temperature (heat removal control), burnable poisons, solid control rods, core configuration, and burnable poison gas ejectors. Each of these options can be designed to function in an autonomous configuration with minimized external actions.

\section{AUTONOMOUS CONTROL STRATEGIES}

The VHTR configurations can be designed with smaller initial excess reactivity values by adjusting graphite content in the system. It is possible to optimize systems varying the carbon-to-heavy metal atom ratio through adjustments in fuel characteristics like TRISO content per compact.

There is a relative trend for last fluence to dommate effective multiplication factor as a factor limiting core lifetime. Because of material properties (graphite and $\mathrm{SiC}$ ) and neutron flux spatial distributions, core lifetime is defined by fast fluence limitations and not as strongly by effective multiplication factor. Changes to $\mathrm{ZrC}$ and reductions in power density help increasing core lifetime values by shifting dominance to effective multiplication factor from fast fluence limitations. [10] Flux peaking reductions facilitate this trend. Figure 3 illustrates behavior of the VHTR core lifetime as a finction of fast fluence.

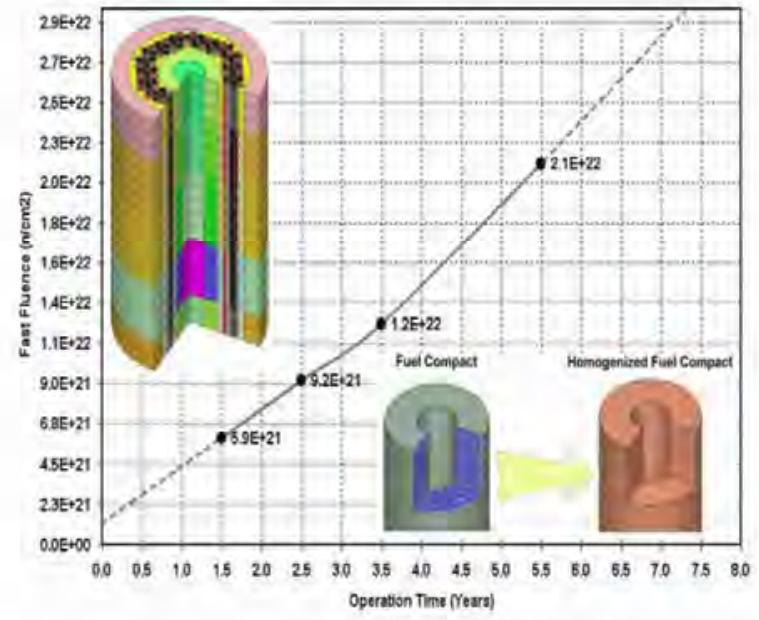

Figure 3. Longevity analysis of the VHTR design configurations as a function of fast fluence.

Fissile fuel inventory requirements are shown in Fig. 4 for various TRU-fueled VHTR configurations relative to the LEUfueled VHTR configuration. [9.] Trade-offs between loading 


\section{Utilization of MAs as a Fuel Component for Ultra-Long Life} VHTR Configurations: Designs, Advantages and Limitations

operations, fuel activity and other factors will ultimately constrain the VHTR design with respect to its TRU content. $[7,8,10]$

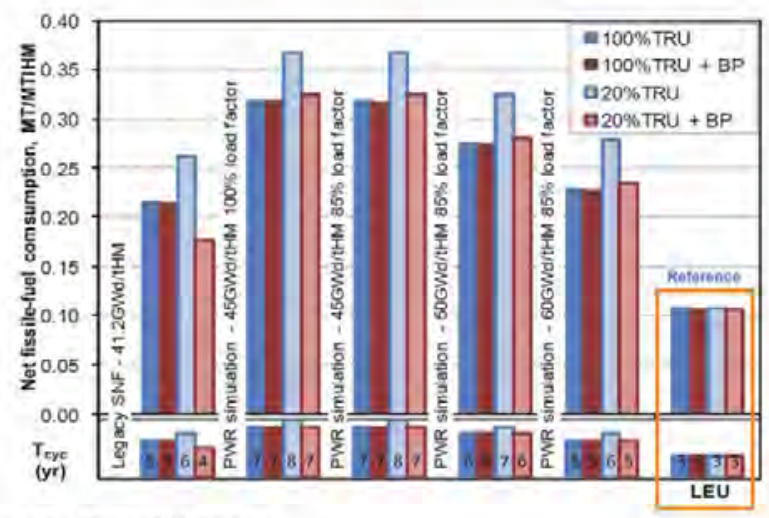

BP - Buruable poison

Figure 4. Fissile content requirements for various TRU-fueled VHIR configurations operating at $600 \mathrm{MW}_{\text {th }}$ relative to the corresponding LEU-fueled VHTR system.

Taking advantage of three burnable poison locations in the HTTR fuel block design, it is possible to reduce initial excess reactivity values in the VHTR configurations. The same three locations also allow controlling reactivity swings during operation. Figure 5 shows the HTTR fuel block model and burnable poison locations as they are used in the present analysis. [1.18.19]

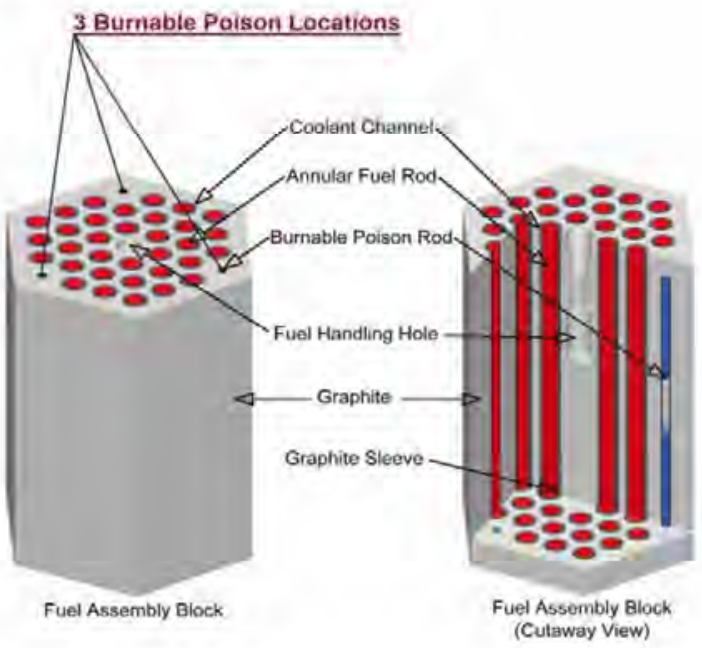

Figure 5. Burnable poison locations in the HTTR fuel block (KENO3D Plot).
The VHTR system response on burnable poison distribution variations is shown in Figure 6. For one of the considered LEU-fueled VHTR configurations, one, two and three burnable poison locations (see Fig. 5) have been utilized sequentially to evaluate distribution effects during operation and identify potential control system needs and requirements.

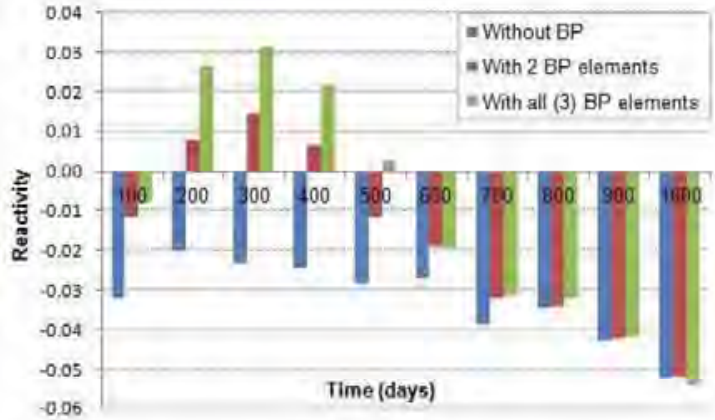

Figure 6. Reactivity swing in the LEU-fueled VHTR configuratious duning operation.

Reactivity swings can passively be coutrolled and optimized during operation by using burnable poison distributions. In the present analysis, heterogeneons. distributions are considered using dedicated absorber locations in the HTTR fiuel block design. Figure 7 illustrates observed self-adjustments in the operation characteristics of the LEUand TRU-fueled VHTR systems as a restlt of system response variations triggered by different burnable poison distributions.

\section{CONCLUSIONS}

In the present analysis, performance characteristics of various burnable poison distributions and fuel compositions in the VHTR core have been evaluated. The basis configuration is developed using Generation IV VHTR core specifications and the HTTR hexagonal block geometry data. Utilization of TRUs in VHTRs is explored as the core self-stabilization approach. The resulting configurations have an inherent potential for autonomous operation with minimized maintenance. Adjustments of heterogeneous bumable poison distributions in the VHTR core leads to substantial reductions in initial excess reactivity levels as well as allows to passively control reactivity swings during operation. Both outcomes facilitate studies of potential deployment options for autonomous control in the VHTR systems with LEU and/or TRUs.

\section{ACKNOWLEDGMENTS}

This paper is based upon work supported by the U.S Department of Energy under Award Number DE-FC0705ID14655 (05-094).

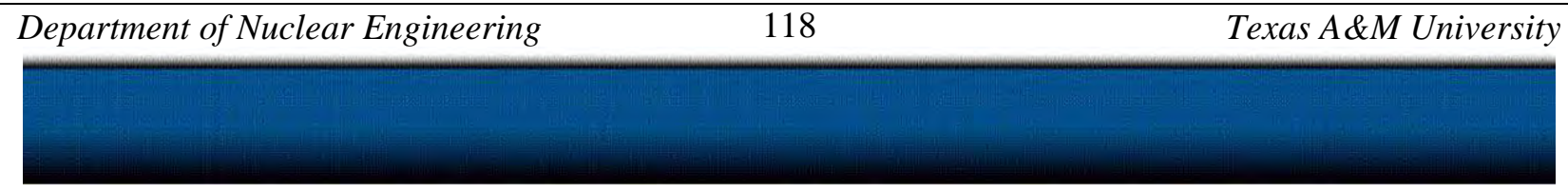




\section{Utilization of MAs as a Fuel Component for Ultra-Long Life} VHTR Configurations: Designs, Advantages and Limitations

\section{Project 05-094}

Final Scientific/Technical Report
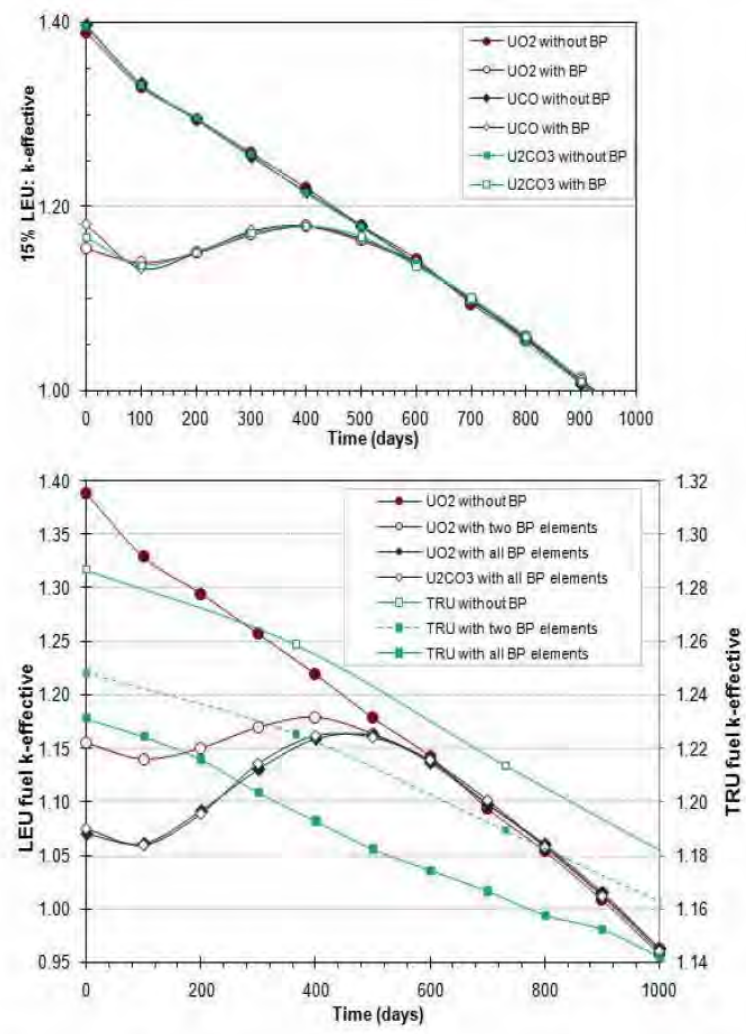

Figure 7. Passive reactivity swing control using heterogeneous burnable poison distributions in the LEU- and TRU-fueled VHTR configurations.

\section{DISCLAIMER}

This paper was prepared as an account of work sponsored by an agency of the United States Government. Neither the United States Government nor any agency thereof, nor any of their employees, makes any warranty, express or implied, or assumes any legal liability or responsibility for the accuracy, completeness, or usefulness of any information, apparatus, product, or process disclosed, or represents that its use would not infringe privately owned rights

Reference herein to any specific commercial product, process, or service by trade name, trademark, manufacturer, or otherwise does not necessarily constitute or imply its endorsement, recommendation, or favoring by the United States Government or any agency thereof.

The views and opinions of authors expressed herein do not necessarily state or reflect those of the United States Government or any agency thereof.

\section{REFERENCES}

1. D. E. AMES II, "Configuration Adjustment Potential of the VHTR Prismatic Cores with Advanced Actinide Fuels", Thesis, Master of Science, August 2006, Nuclear Engineering, Texas A\&M University (2006).

2. G. B. BRUNA et al., "Uncertainty Analysis and Optimization Studies on the Deep-Burner-Modular Helium Reactor (DB-MHR) for Actinide Incineration", Proc. PHYSOR2004 - The Physics of Fuel Cycle and Advanced Nuclear System: Global Developments, Chicago, USA, April 25-29, 2004, American Nuclear Society, Lagrange Park, IL. (2004).

3. "Critical Experiments and Reactor Physics Calculations for Low-Enriched HTGRs", IAEA-TECDOC-1249, International Atomic Energy Agency, Vienna, Austria (2001).

4. "Evaluation of High Temperature Gas-cooled Reactor Performance: Benchmark Analysis Related to Initial Testing of the HTTR and HTR-10", IAEA-TECDOC1382, International Atomic Energy Agency, Vienna, Austria (2003).

5. "Final Environmental Impact Statement for a Geologic Repository for the Disposal of Spent Nuclear Fuel and High-Level Radioactive Waste at Yucca Mountain, Nye County, Nevada", Vol. 2, Appendix A, "Inventory and Characteristics of Spent Nuclear Fuel, High-Level Radioactive Waste, and Other Materials", DOE/EIS-0250, U.S. DOE (2002).

6. "Generation IV Nuclear Energy Systems: Ten Year Program Plan", March 2005, Office of Advanced Nuclear Research, DOE Office of Nuclear Energy, Science, and Technology, U.S. DOE (2005)

7. T. KIM, T. TAIWO, R. HILL, W. YANG, F. VENNERI, "A Feasibility Study of Reactor-Based Deep-Burn Concepts", ANL-AFCI-155, Argonne National Laboratory (2005).

8. K. KUNITOMI, "Development of New Type of HTGR", Proc. 73rd JSME Fall Annual Meeting, JSME, Japan, 1995.

9. T. LEWIS, D. AMES, P. TSVETKOV, "Analysis of TRUFueled VHTR Prismatic Core Performance Domains", Trans. Amer: Soc., 97, 859 (2007).

10. K. MINATO, T. OGAWA, K. SAWA, A. ISHIKAWA, T. TOMITA, S. IIDA, H. SEKINO, "Irradiation Experiment on $\mathrm{ZrC}$-Coated Fuel Particles for High Temperature GasCooled Reactors", Nucl. Techn., 130, 272 (2000).

11. K. MINATO, K. SAWA, T. KOYA, T. TOMITA, A. ISHIKAWA, "Fission Product Release Behavior of Individual Coated Fuel Particles for High Temperature Gas-Cooled Reactors", Nucl. Techn., 131, 36 (2000).

12. C. M. N. A. PEREIRA, "Evolutionary Multicriteria Optimization in Core Design: Basic Investigations and Case Study", Annals of Nuclear Energy, 31, 1251-1264 (2004).

13. R. PLUKIENE, D. RIDIKAS, "Modeling of HTRs with Monte Carlo: from a Homogeneous to an Exact 


\section{Utilization of MAs as a Fuel Component for Ultra-Long Life VHTR Configurations: Designs, Advantages and Limitations}

Heterogeneous Core with Microparticles", An. Nucl. En., 30, 1573, (2003).

14. "Report to Congress on Advanced Fuel Cycle Initiative: The Future Path for Advanced Spent Fuel Treatment and Transmutation Research", 03-GA50439-06, January, 2003, Office of Nuclear Energy, Science, and Technology, U.S. DOE (2003).

15. C. RODRIGUEZ, A. BAXTER, D. MCEACHERN, M. FIKANI, F. VENNERI, "Deep-Burn: Making Nuclear Waste Transmutation Practical", Nucl. Eng. Design, 2805(1) (2003).

16. "SCALE: A Modular Code System for Performing Standardized Computer Analyses for Licensing Evaluation", ORNL/TM-2005/39,Ver.5.1, November 3, 2006, Oak Ridge National Laboratory, Tennessee (2006).

17. T. A. TAIWO, T. K. KIM, W. S. YANG, H. S. KHALIL, "Evaluation of High Temperature Gas-Cooled Reactor Physics Experiments as VHTR Benchmark Problems", ANL-GenIV-059, Argonne National Laboratory, September 2005.

18. A. TALAMO, "Managing the Reactivity Excess of the Gas Turbine-Modular Helium Reactor by Burnable Poison and Control Rods", Ann. Nucl. Energy, 31, 84-98 (2006).

19. A. TALAMO, "Conceptual Design of QUADRISO Particles for High Temperature Reactors", Trans. Amer: Nucl. Soc. and Embedded Topical Meetings on Isotopes for Medicine and Industry and Nuclear Fuels and Structural Materials for the Next Generation Nuclear Reactors, 98. 727-728 (2008).

20. P. TSVETKOV, A. ALAJO, D. AMES, M. PRITCHARD, "Spectrum Shifting as a Mechanism to Improve Performance of VHTRs with Advanced Actinide Fuels", Proc. 14th Intern. Conf. Nucl. Eng. (ICONE 14), Miami, Florida, July 17 - 20, 2006, ASME (2006).

21. H. N. TRAN, Y. KATO, "An Optimal Loading Principle of Bumable Poisons for an OTTO Refueling Scheme in Pebble Bed HTGR Cores", Proc. 2008 Intern. Congress on Advances in Nucl. Power Plants, Embedded Topical Meeting, June 8-12, 2008, Anaheim, California, USA, 289297 (2008)

22. P. V. TSVETKOV, D. E. AMES II, M. L. PRITCHARD, A. B. ALAJO, T. G. LEWIS III, "TRU-Fueled VHTRs: Design, Performance, and Applications", Proc. GLOBAL2007: Advanced Fuel Cycles and Systems, September 9-13, 2007, Boise, Idaho, 852-855, USA, (2007).

23. P. V. TSVETKOV, T. G. LEWIS III, A. B. ALAJO, "TRUFueled VHTRs for Applications Requiring an Extended Operation with Minimized Control and No Refueling", Proc. $16^{\text {th }}$ Intern. Conf. Nucl. Eng. (ICONE 16), Orlando, Florida, May 11-15, 2008, ASME (2008). 


\title{
6.11. "Used Fuel" Vectors and Waste Minimization Strategies for VHTRs Operating without Refueling
}

\author{
Pavel V. Tsvetkov, Tom G. Lewis III, Ayodeji B. Alajo, David E. Ames II \\ Department of Nuclear Engineering \\ Texas A\&M University \\ 129 Zachry Engineering Center, MS 3133 TAMU, College Station, Texas 77843-3133, USA \\ Phone: $979 / 845-7078$, \\ Fax: $979 / 845-6443$ \\ E-mail: Tsvetkov@tamu.edu
}

\begin{abstract}
Generation IV Very High Temperature Reactors (VHTRs) are well-kuown for their llexibility witl respect to feasible fuel cycle options. In this paper, the LEU- and TRU-fueled VHTR configurations are analyzed accounting for their capabilities to attain an extended single-batch OTTO (Once-Throngh-Then Out) mode of operation without intermediate refieling. The requirement of waste minimization is imposed as one of the design constraints defining possible system configurations and deployment strategies. The resulting "used fiel" vectors are examined considering antieipated disposal options as well as viability of fuel reprocessing. A Monte Carlo-deterministic analysis methodology has been implemented for coupled design studies of VHTRs with TRUs using the ORNL SCALI 5.1 code system. The developed modeling approach provides an exact-geometry $3 \mathrm{D}$ representation of the VHTR core details properly captuing VHTR physics. The presented analysis is focused on prismatic block core concepis for VHTRs. It is being performed within the scope of the U.S. DOE NER project ou utilization of higher actuides (TRUs and partitioned MAs) as a fuel component for extended-life VHTR configurations.
\end{abstract}

\begin{tabular}{|c|c|}
\hline \multicolumn{2}{|c|}{ NOMENCLATURE } \\
\hline HTGR & - High Temperature Gas-cooled Reactor \\
\hline HTTR & - High Temperature Test Reactor \\
\hline LEU & - Low-Emriched Uranium \\
\hline LWR & - Light Water Renctor \\
\hline MA & - Minor Actinides \\
\hline NERI & - Nuclear Energy Research Initiative \\
\hline OTTO & - Once-Through-Then-Out \\
\hline TRU & - Transuranium Nuclides \\
\hline VHTR & - Very High Temperature Reactor \\
\hline
\end{tabular}

\section{INTRODUCTION}

Generation IV Very High Temperature Reactors (VHTRs) are well-known for their flexibility with respect to feasible fuel cycle options. $[2,15]$ In this paper, the LEU- and TRU-fueled VHTR configurations are analyzed accounting for fheir capabilities to antain an extended single-batch OITO (OnceThrough-Then -Out) mode of operation without intermediate refueling. $[8,19]$

The envisioned LWR-VHTR fuel cycle with TRU-recycle is assiumed to yield only fission products for disposal. In this scheme, PWR fuel elements with $3,75 \%$ LEU, which were burnt up to 41,200 MWdimtHM and cooled for 23 years, are being reprocessed. [5] Figure 1 shows nuclear physics characteristics of the recovered TRU vectors after 23 years of cooling.

Partitioned TRU and depleted uranium (DU) can be recycled in VHTRs and potentially in PWRs. When fast reactors (FR) become viable, the suggested fuel cycle scheme may be transformed into a sustainable nuclear energy system that does not require a LEU-teed. It is expected that LWRs will eventually be replaced by Generation IV VHTRs. [6] At that time sustamable nuclear systems consisting of FRs and VHIRs will become possible [7,14.15] Shifting fresh fuel vectors for VHTRs towards higher actinides (TRISO fuels with a high TRU-content) stabilizes in-core firel cycles of VHTRs. As a result, single-loading lifetimes of VHTRs with TRUs can be extended np to temporal limits of core materials. It is assumed that the single batch lifetime in the VHTR core is determined by attained fast fluence levels in TRISO coatings. The ultimate idea is to approach autonomous operation that would allow marketing power units with VHIRs as muclear batleties for worldwide deployment. The requirement of waste

\begin{tabular}{lll}
\hline Department of Nuclear Engineering & 121 & Texas A\&M University \\
\hline & &
\end{tabular}




\section{Utilization of MAs as a Fuel Component for Ultra-Long Life VHTR Configurations: Designs, Advantages and Limitations}

minimization is imposed as one of the design constraints defining possible system configurations and deployment strategies. The single-batch OTTO mode is suitable for VHTRs designed as internationally deployable systems for operation conditions requiring minimized maintenance. As a goal for such conditions, the "battery-type" systems are studied assuming modular design and core-cartridge/reactor unit replacement strategies. The resulting "used fuel" vectors are examined considering anticipated disposal options as well as viability of fuel reprocessing. [8]

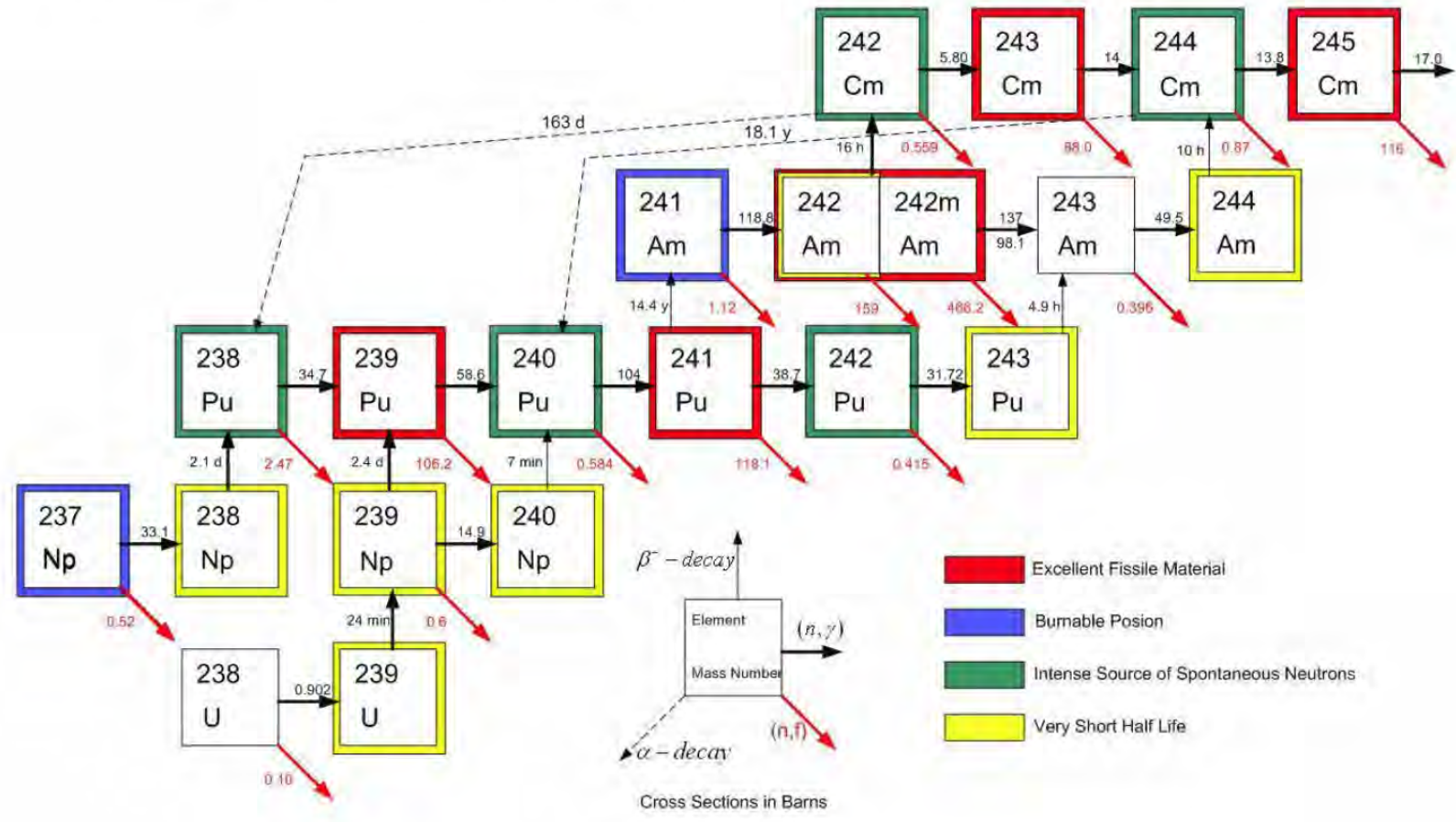

TRU vector (PWR 41.200 MWd/tHM, decay=23 years), at. \%:

237-Np- 6.121:

238-Pu - 1.986, 239-Pu - 51.718, 240-Pu - 21.899, 241-Pu - 4.104, 242-Pu - 4.451

241-Am- 8.250,242m-Am-0.020, 243-Am - 1.230

$243-\mathrm{Cm}-0.003,244-\mathrm{Cm}-0.194,245-\mathrm{Cm}-0.021,246-\mathrm{Cm}-0.003$

Figure 1. Nuclide transformations in TRU vectors recovered from a typical PWR spent fuel after 23 years of cooling.

A Monte Carlo-deterministic analysis methodology has been implemented for coupled design studies of VHTRs with TRUs using the ORNL SCALE 5.1 code system. [16] The developed modeling approach provides an exact-geometry $3 \mathrm{D}$ representation of the VHTR core details properly capturing VHTR physics.

The presented analysis is focused on prismatic block core concepts for VHTRs. It is being performed within the scope of the U.S. DOE NERI project on utilization of higher actinides (TRUs and partitioned MAs) as a fuel component for extendedlife VHTR configurations. The research focus is on possible designs and their advantages and limitations. [9,18-19]

\section{MODELING APPROACH \& ANALYSIS STRATEGIES}

In the context of the U.S. DOE NERI project, the VHTR system is modeled accounting for physics details of VHTRs. secondary side, potential applications, fuel cycle, and environmental impact. The VHTR system representation procedure includes multi-physics and assessments of performance characteristics. As a result, the applied system analysis approach allows considering the VHTR fleet model (reactors and fuel cycle) as a complex system that consists of multiple interacting components structured hierarchically and exhibiting common behavior. [12] It allows identifying significant components, global characteristics, energy flows 
Utilization of MAs as a Fuel Component for Ultra-Long Life VHTR Configurations: Designs, Advantages and Limitations

and conversion stages, system boundary interactions and interfaces including environmental impacts, and safety characteristics. The modeling approach is shown in Fig. 2.

The use of Monte Carlo methods creates a potential for explicit 3D whole-core/reactor studies of the VHTR configurations addressing the key physics areas. Figure 3 shows the developed 3D VHTR model for use in calculations with the ORNL SCALE 5.1 code system. [16]

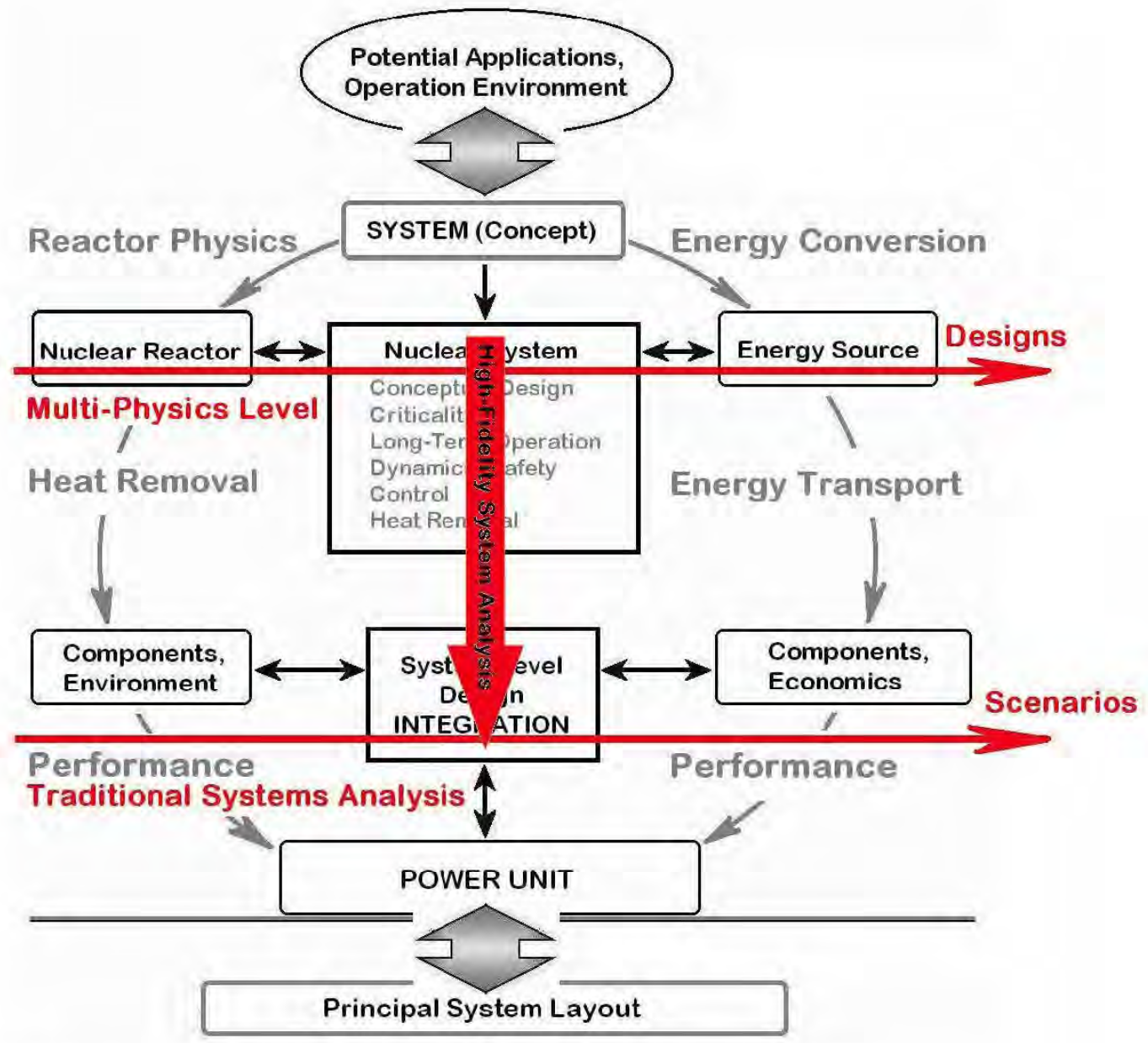

Figure 2. High fidelity system modeling approach and analysis strategies for TRU-fueled VHTRs

The basis configuration has been developed using the Generation IV VHTR core specifications and the HTTR hexagonal block geometry data. [3,4,6] The basis design parameters ( $\mathrm{UO}_{2}$ - configuration) are summarized in Table 1.

The model is a near-exact representation of the expected physical description of the VHTR configuration. The computational scheme combines the available capabilities of the SCALE Monte Carlo (whole-core analysis level, KENO) and deterministic functional modules (lattice level).
The system performance metrics can be introduced considering in-core and out-of-core fuel cycle performance characteristics independently. Because the present analysis is focused on maximizing longevity of VHTRs, the in-core fuel cycle performance metrics naturally include core lifetime, effective multiplication factor, fast fluence, reactivity coefficients, power peaking factors, core geometry, power output, and other core parameters. Figure 4 illustrates how the TRU-fueled VHTR system performance domain is identified. 
Utilization of MAs as a Fuel Component for Ultra-Long Life VHTR Configurations: Designs, Advantages and Limitations

Project 05-094

Final Scientific/Technical Report

\begin{tabular}{|c|c|c|c|}
\hline Fuel & $\mathrm{UO}_{2}$ & Power $\left(\mathrm{MW}_{\mathrm{tib}}\right)$ & 600 \\
\hline Enrichment (\%) & 9 & Power Density (W/ $\left./ \mathrm{cm}^{3}\right)$ & 6.9 \\
\hline \multirow[t]{2}{*}{ Coolant } & \multirow[t]{2}{*}{$\mathrm{He}$} & Pressure (MPa) & 7.0 \\
\hline & & Inlet/Outlet Temperature $\left({ }^{\circ} \mathrm{C}\right)$ & $490 / 950$ \\
\hline \multirow[t]{3}{*}{ \# of Columns } & \multirow[t]{3}{*}{102} & \# of Fuel Colunns & 66 \\
\hline & & \# of Control Columns & 36 \\
\hline & & $\#$ of Blocks/Column & 13 \\
\hline Block Pitch $(\mathrm{cm})$ & 36 & \# of Fuel Pins/Fuel Block & 32 \\
\hline \multirow[t]{7}{*}{ Block Height $(\mathrm{cm})$} & \multirow[t]{7}{*}{58} & \# of $\mathrm{B}_{4} \mathrm{C}$ Rods/Fuel Block & 2 \\
\hline & & Control Rods/Control Block & 2 \\
\hline & & Emergency Rods/Control Block & 1 \\
\hline & & Compact Pitch (cm) & 5.15 \\
\hline & & Fuel Hole Radius $(\mathrm{cm})$ & 4.1 \\
\hline & & Compact Inner Radius $(\mathrm{cm})$ & 0.5 \\
\hline & & Compact Outer Radius (cm) & 1.3 \\
\hline \multirow[t]{7}{*}{ Packing (\%) } & \multirow[t]{7}{*}{30} & $10.41 \mathrm{~g} / \mathrm{cm}^{3}$ Kentel Radius $(\mathrm{cm})$ & 0.02985 \\
\hline & & $1.14 \mathrm{~g} / \mathrm{cm}^{2}$ Buffer Radius (cm) & 0.03588 \\
\hline & & $1.89 \mathrm{~g} / \mathrm{cm}^{3} \mathrm{PyCl}$ Radius $(\mathrm{cm})$ & 0.03895 \\
\hline & & $3.20 \mathrm{~g} / \mathrm{cm}^{3} \mathrm{SiC}$ Radius $(\mathrm{cm})$ & 0.04184 \\
\hline & & $1.87 \mathrm{~g} / \mathrm{cm}^{3} \mathrm{PyC} 2$ Radius (cmi) & 0.04645 \\
\hline & & Matrix $\left(\mathrm{g} / \mathrm{cm}^{3}\right)$ & 1.77 \\
\hline & & Block $\left(\mathrm{g} / \mathrm{cm}^{3}\right)$ & 1.69 \\
\hline
\end{tabular}

Consequently, the out-of-core fuel cycle performance metrics could be defined in terms of fuel utilization characteristics including waste minimization constraints: Beginning-of-Life (BOL) fuel loading and composition, Endof-Life (EOL) fuel loading and composition, BOL and EOL activities of the fuel composition, BOL and EOL heat rates of the fuel composition. BOL and EOL fissile inventories, BOL and EOL fertile inventories, and transmutation efficiency.

\section{VHTR MODEL BENCHMARKING}

The adequacy of the applied computational methods and models used to evaluate performance is supported by comparisons with experimental data covering appropriate ranges of conditions. As the basis for the benchmark test problems, the project uses the actual test results and benchmark problems of the HTTR Program (prismatic core design, reactor experiments). $[3,4]$
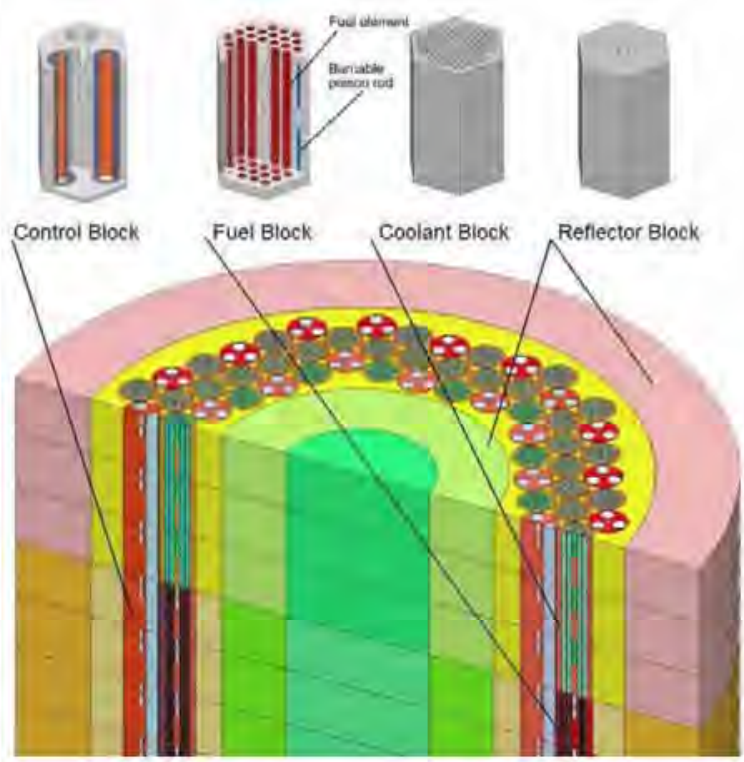

Figure 3. 3D whole-core exact-geometry VHTR model (KENO3D Plot).

The results of the analysis for the HTTR benchmark configuration showing comparison to experimental data are summarized in Table 2. [1]

Table 2. Experiment-to-Code Model Validation

\begin{tabular}{|l|l|l|l|l|}
\hline \multicolumn{2}{|l|}{ Case } & $\begin{array}{l}\text { VHTR } \\
\text { (SCALE5.1) }\end{array}$ & $\begin{array}{l}\text { HTTR } \\
\text { (Test) }\end{array}$ & $\begin{array}{l}\text { Error } \\
(\%)\end{array}$ \\
\hline Control Rods Out & $k_{e f f}$ & $1.137 \pm 0.002$ & $1.14 \pm 0.04$ & 0.044 \\
\hline Control Rods In & $k_{\text {ef }}$ & $0.686 \pm 0.002$ & $0.69 \pm 0.01$ & 0.117 \\
\hline Critical Insertion (300K) & $\mathrm{cm}$ & 177.1 & $177,5 \pm 0.5$ & 0.225 \\
\hline Critical Insertion (418K) & $\mathrm{cm}$ & 189.9 & $190.3 \pm 0.5$ & 0.210 \\
\hline Temperature Coefficient & $1 / \mathrm{K}$ & $-1.45 \mathrm{E}-04$ & $-1.42 \mathrm{E}-04$ & 2.113 \\
\hline
\end{tabular}

The VHTR model is a nearly explicit representation of the existing HTTR core configuration. The model was created in the SCALE code system utilizing the CSAS6/KENO-VI module, which allows flexible geometry representations. The geometry package in KENO-VI is capable of modeling any volume that can be constructed by quadratic equations (second order surfaces).

Obtained benchmark results are in agreement with the available HTTR data and confirm applicability of the chosen modeling methodology. Each of the benchmark cases is within $0.25 \%$ of the experimental values and fall within experimental error. The computed value of the isothermal temperature coefficient deviates by about $2 \%$ from the corresponding

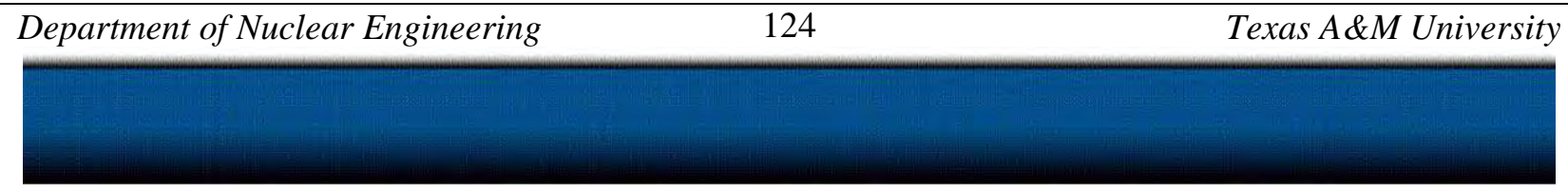




\section{Utilization of MAs as a Fuel Component for Ultra-Long Life} VHTR Configurations: Designs, Advantages and Limitations

experimental value. However, the experimental value is within the standard deviation limits of the computational result.

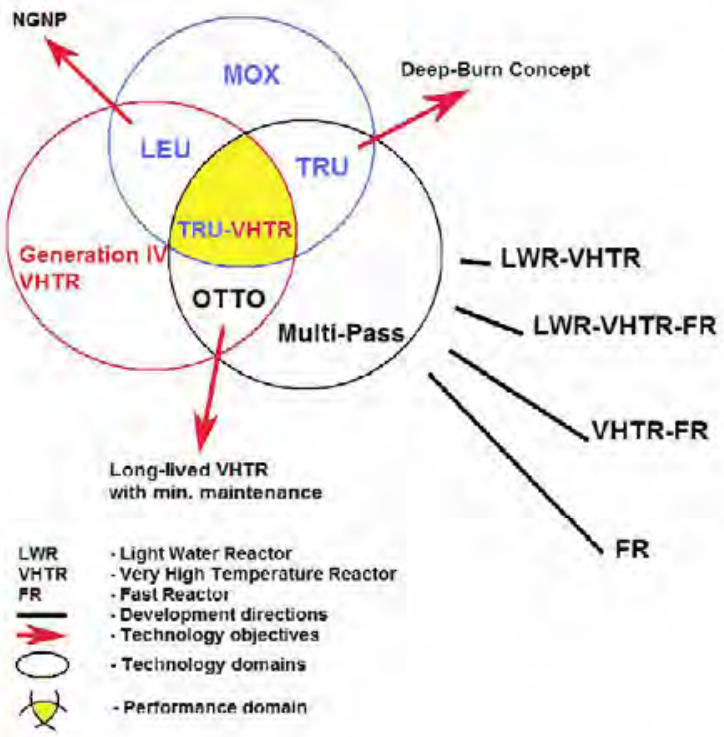

Figure 4. Performance domain identification process.

\section{PHYSICS FEATURES OF VHTR CONFIGURATIONS}

The use of coated micro-particles creates doubleheterogeneity that defines performance characteristics of the VHTR core. The first level of heterogeneity (micro-level) is formed by coated fuel micro-particles that are randomly distributed in the graphite compact. The second level of heterogeneity (macro-level) is formed by the prismatic blocks with compacts that compose the VHTR core. For desirable performance characteristics, the prismatic graphite block core has channels for coolant, instrumentation and handling operations. As a result, there are various neutron streaming passages formed by voids, coolant channels, instrumentation and handling channels, plenums, and etc. The proximity to outer and central reflector regions is also an important factor that influences the VHTR physics. Depending on a specific computational model, these features act and can be taken into account as multiple additional levels of heterogeneity up to the $3 \mathrm{D}$ finite core model that provides an appropriate comprehensive multi-heterogeneity treatment. This principal arrangement creates phenomena complicating analysis of these systems. It is apparent that the double-heterogeneity effects should be expected to play a defining role for all nuclides in the VHTR cores, especially those with resonance cross sections, including fuel nuclides like ${ }^{235} \mathrm{U}$ and ${ }^{238} \mathrm{U}$ and burnable absorbers like erbium. $[13,17]$ Furthermore, as compared to the exact geometry modeling using Monte Carlo codes, the simplified single-level macro-heterogeneity modeling could significantly over estimate fuel lifetimes in VHTRs. [13]

At the same time, it gives flexibility in fuel selection. management core performance and adaptability to the changing economics. It has already been demonstrated in basic reactor physics parameter studies that the same reactivity variation can be attained as a result of different changes of the basic VHTR core parameters. Table 3 illustrates the effect of carbon-to-heavy metal ( $\mathrm{C} / \mathrm{HM})$ atom ratio variations on the VHTR physies characteristics. The $\mathrm{C} / \mathrm{HM}$ variations are achieved by adjusting numbers of TRISO particles per compact.

Table 3. VHTR Core Effects due to C/HM-Variations

\begin{tabular}{|l|l|l|l|l|l|l|l|}
\hline $\begin{array}{l}\text { Fissile Atom } \\
\text { Fraction }\end{array}$ & \multicolumn{3}{|c|}{ C/HM } & \multicolumn{2}{c|}{$k_{\text {eff }}$} & \multicolumn{2}{c|}{ EALF (eV) } \\
\hline LEU & TRU & LEU & TRU & LEU & TRU & LEU & TRU \\
\hline 9.00 & 53.06 & 249 & 1271 & 0.74 & 1.00 & - & 0.21 \\
\hline 9.00 & 53.06 & 121 & 845 & 0.98 & 1.05 & - & 0.23 \\
\hline 9.00 & 53.06 & 79 & 632 & 1.09 & 1.08 & 0.17 & 0.25 \\
\hline 9.00 & 53.06 & 57 & 505 & 1.15 & 1.08 & 0.19 & 0.27 \\
\hline 9.00 & 53.06 & 36 & 419 & 1.21 & 1.08 & 0.23 & 0.29 \\
\hline 9.00 & 53.06 & 25 & 359 & 1.24 & 1.08 & 0.29 & 0.31 \\
\hline 9.00 & 53.06 & 10 & 313 & 1.26 & 1.08 & 0.57 & 0.34 \\
\hline- & 53.06 & - & 249 & - & 1.07 & - & 0.39 \\
\hline- & 53.06 & - & 207 & - & 1.06 & - & 0.46 \\
\hline- & 53.06 & - & 176 & - & 1.06 & - & 0.53 \\
\hline- & 53.06 & - & 153 & - & 1.05 & - & 0.61 \\
\hline- & 53.06 & - & 79 & - & 1.04 & - & 1.10 \\
\hline- & 53.06 & - & 25 & - & 1.10 & - & 8.69 \\
\hline- & 53.06 & - & 12 & - & 1.17 & - & 32.77 \\
\hline
\end{tabular}

EALF - average neutron lethargy causing fission

$\mathrm{C} / \mathrm{HM}=$ Carbon-to-heavy-metal atom ratio

Figure 5 illustrates the corresponding spectral effects in VHTRs due to $\mathrm{C} / \mathrm{HM}$ atom ratio variations. For comparable $\mathrm{C} / \mathrm{HM}$ ratios in the critical LEU- and TRU-fueled configurations, the VHTR system with TRUs does not have a distinct thermal peak. For C/HM ratios below approximately 100 in the TRU-fueled VHTR configurations, thermalization effects in compact matrix disappear. Such configurations could prove more difficult to control and require further safety analysis. 


\section{Utilization of MAs as a Fuel Component for Ultra-Long Life} VHTR Configurations: Designs, Advantages and Limitations

\section{Project 05-094}

Final Scientific/Technical Report

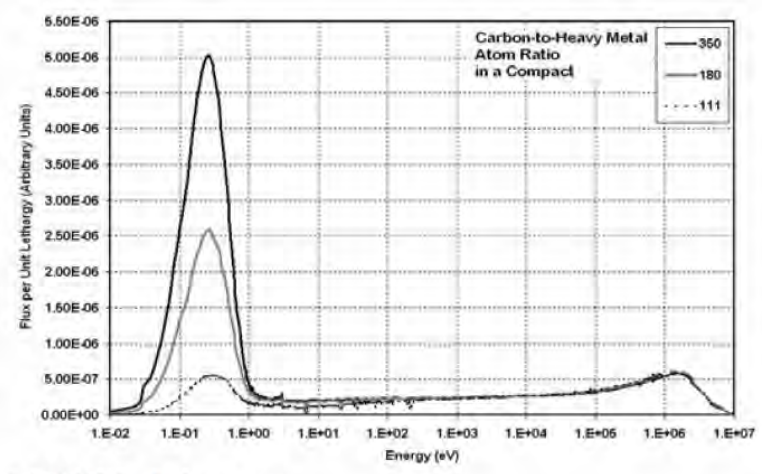

(a) LEU-fueled systems

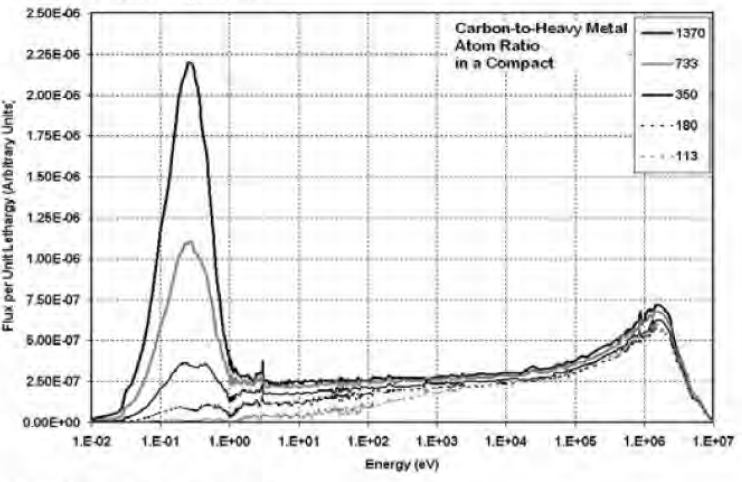

(b) TRU-fueled systems

Figure 5. Spectral effects of $\mathrm{C} / \mathrm{HM}$-variations in the VHTR configurations.

Table 4 links $\mathrm{C} / \mathrm{HM}$ atom ratio variations in the TRUfueled VHTR configurations to the configuration longevity and destruction of TRUs. It can be observed that the VHTR configuration lifetime can be modified by adjusting such TRISO fuel design parameters as TRISO loading per compact or compact dimensions (amount of matrix graphite per compact).

The core lifetime is clearly limited by fast neutron fluences. Fast fluence levels attained by TRISO particles in the LEU- and TRU-fueled VHTR configurations with the longest lifetimes with respect to criticality are around $7 \times 10^{22} \mathrm{n} / \mathrm{cm}^{2}$. Therefore, expected fast fluences in the longest-operating VHTR configurations approach and may potentially exceed the traditional TRISO fast fluence limitations of about $10^{26} \mathrm{n} / \mathrm{m}^{2}$. This constraint can be relaxed or even eliminated by reducing power density levels and using advanced radiation-tolerant materials for extended-life VHTR configurations. Advanced coating configurations and materials like $\mathrm{ZrC}$ offer promising performance characteristics including reductions of coating failure probabilities yielding fission product releases. $[10,11]$ Consequently, higher fast fluence limits, and hence longer core lifetimes, may become acceptable approaching criticality core lifetime limitations. Safety characteristics of the TRU-fueled VHTRs retain their acceptable values during considered core lifetimes.

Table 4. TRU-VHTR Core Effects due to C/HM-Variations

\begin{tabular}{|l|l|l|}
\hline C/HM & $\begin{array}{l}\text { VHTR Lifetime } \\
\text { (OTTO) (years) }\end{array}$ & $\begin{array}{l}\text { TRU Destruction } \\
\text { (\% mtHM) }\end{array}$ \\
\hline 9 & 9 & 33.62 \\
\hline 11 & 8 & 29.86 \\
\hline 23 & 7 & 26.03 \\
\hline 33 & 6 & 22.26 \\
\hline 53 & 6 & 22.21 \\
\hline 112 & 5 & 18.44 \\
\hline 229 & 2 & 7.36 \\
\hline
\end{tabular}

\section{EXTENDED SINGLE-BATCH OTTO MODE}

As above emphasized, the use of coated micro-particles creates gives flexibility in fuel selection, management, core performance and adaptability. The VHTR multi-heterogeneity features allow material separation by using different types of micro-particles and graphite blocks. The degree of heterogeneity and the moderator-to-fuel ratio $(\mathrm{C} / \mathrm{HM}$ atom ratio) can be adjusted to achieve desirable spectrum shifting. It should be possible to enhance properties of some TRUs by neutron spectrum shifting through configuration adjustments. [1]

In this study, the LEU- and TRU-fueled VHTR configurations are analyzed accounting for their capabilities to attain an extended single-batch OTTO (Once-Through-Then Out) mode of operation without intermediate refueling. The principal mechanism being envisioned to achieve extended-life systems is an enhanced involvement of self-generated fissile compositions based on LWR spent fuel vectors to the energy generation process. Depending on neutron spectra in the considered core configurations (dominant neutron energies), neptunium, americium and curium may serve as burnable poisons or fuel materials contributing to self-stabilization over prolonged irradiation periods. Figure 6 illustrates the ability of VHTRs to attain the extended single-batch OTTO mode. All considered VHTR configurations were analyzed assuming their operation at $103 \mathrm{MW} / \mathrm{mtHM}$. The LEU-fueled configuration utilizes about $75 \%$ of the initially loaded fissile content over 3 year operation. All TRU-fueled configurations demonstrate smaller reductions over much longer lifetimes.

Operation of the TRU-fueled VHTRs is characterized by longer operational times (lifetime with respect to criticality) and potentially smaller reactivity swings during a single batchmode operation. Therefore, transitioning from LEU-systems to VHTR configurations with TRUs allows prolonging operation without refueling up to almost a decade-long OTTO mode. 
Utilization of MAs as a Fuel Component for Ultra-Long Life VHTR Configurations: Designs, Advantages and Limitations

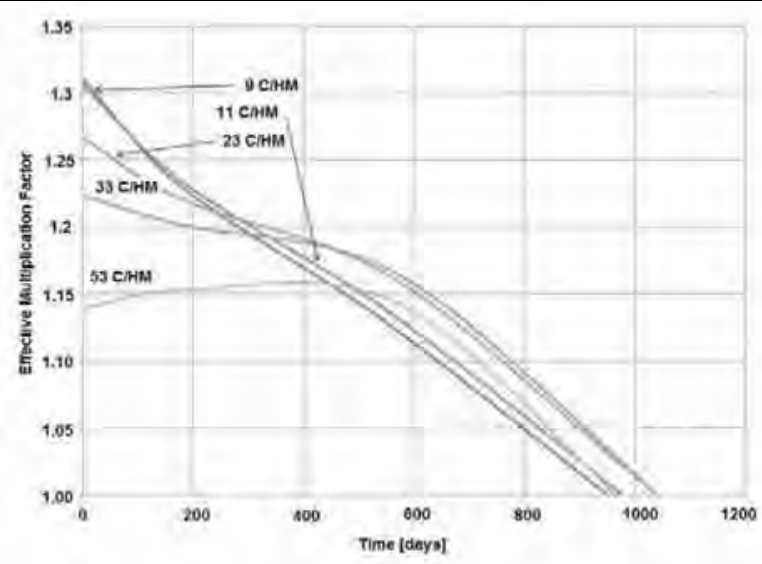

(a) $15 \%$ LEU-fueled systems

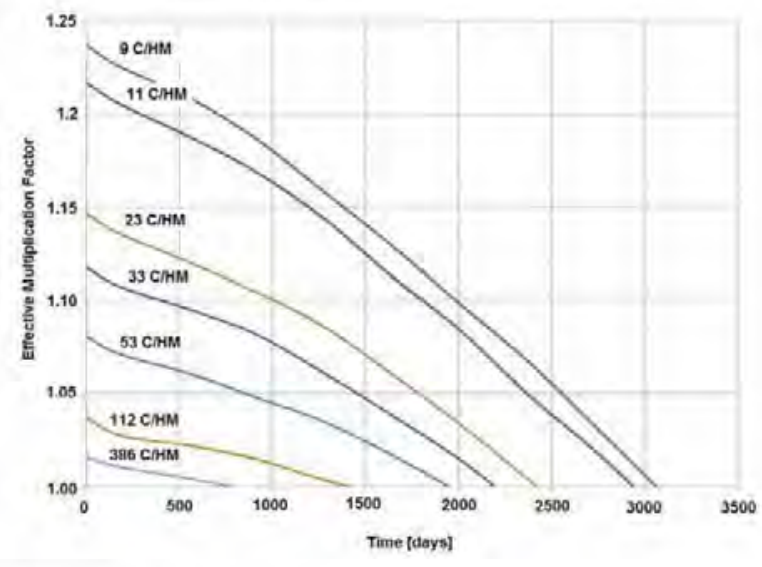

(b) TRU-fueled systems

Figure 6. Extended single-batch OTTO modes of the LEU-and TRU-fieled VHTR configurations.

\section{VHTR "USED FUEL" AND WASTE MINIMIZATION}

In the present analysis, five TRU vectors are considered as potential fuel compositions for VHTRs:

- TRU1: Legacy spent muclear fiel: average burnup of $41,2 \mathrm{GWd} / \mathrm{mrHM}$

- TRU 2: PWR spent fuel at $45 \mathrm{GWd} / \mathrm{m}$ HMM burnup and $100 \%$ load lactor.

- TRU3: PWR spent fuel at $45 \mathrm{GWd} / \mathrm{mtHM}$ burnup and $85 \%$ load factor.

- TRU4: PWR spent fuel at $50 \mathrm{GWd}$ /mHM burnup and $85 \%$ load factor.

- TRU 5: PWR spent fuel at $60 \mathrm{GWd} / \mathrm{mtHM}$ burnup and $85 \%$ load factor.

The differences between TRU vectors are shown in Fig. 7. The first vector, TRU1, represents the legacy PWR fiel with 23 year cooling time (see Fig. 1).

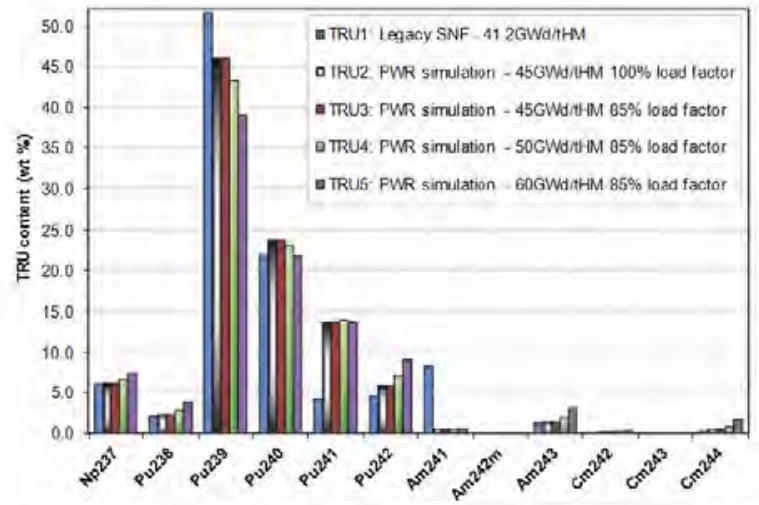

Figure 7. TRU vectors from the PWR spent fuel inventories.

Figure \& summarizes ransmutation efficiencies, destruction of PU, MA, and IRU, as well as evaluations of material inventories for disposal. There are turee waste minimization strategies for the LET- and TRU-fiveled VHTRs to be explored in future studies in detail:

- Extended single-batch OTIO mode (no fuel shuffling, no refueling, no composition optimization) will cooling time prior to HLW disposnl.

- Optimized extended OTTO mode with maximized core lifetime and minimized fast fluence (fuel shufling, no refueling, no composition optimization) 


\section{Utilization of MAs as a Fuel Component for Ultra-Long Life} VHTR Configurations: Designs, Advantages and Limitations

- Optimized extended mode with maximized core lifetime and minimized fast fluence (fuel shuffling, refueling).

- Fresh fuel composition optimization.
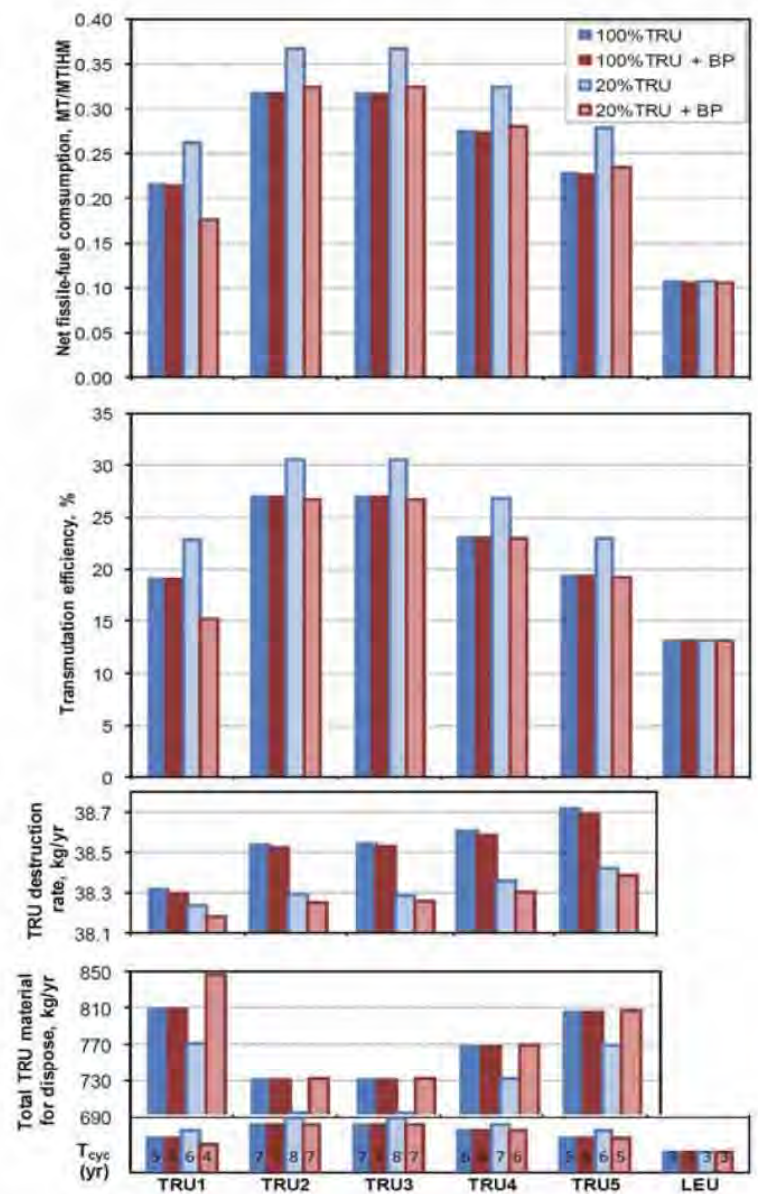

Figure 8. Out-of-core fuel cycle characteristics of the TRUfueled VHTR configurations.

The first strategy is the reference direction. The strategies involving optimization require extra fuel handling operations. Each of the waste minimization strategies may involve fresh fuel composition optimization options. The equivalence analysis should be performed to compare the TRU-fueled VHTRs to LEU-fueled VHTRs and to conventional LWR options with $\mathrm{UO}_{2}, \mathrm{MOX}$ and transmutation. The studies should also include environmental impact assessment as shown in Table 5 .
Table 5. TRU HLW Characteristics for the Longest-Lived TRU-Fueled VHTR Configuration

\begin{tabular}{|l|l|}
\hline Requirement & $\begin{array}{l}\text { Time } \\
\text { (years) }\end{array}$ \\
\hline Time Required To Reach Same Energy Release per Fuel Volume & 71 \\
\hline Time Required To Reach Same Energy Release per MTHM & 164 \\
\hline Time Required To Reach Same Activity per Fuel Volume & 10 \\
\hline Time Required To Reach Same Energy Release per MTHM & 23 \\
\hline
\end{tabular}

HLW - High Level Waste

When the VHTR spent fuel is expected to be sent to the Yucca Mountain Geological Repository, it is going to be assessed on two factors. First, before a waste form can enter the subsurface environment, the waste package has to fall below a specific heat emission requirement. The second and the most important criterion is activity of the HLW. As illustrated in Table 5, for the TRU-fueled VHTR spend fuel to reach the same activity per fuel volume as in the initial loading, the required cooling time is about 10 years.

\section{CONCLUSIONS}

The analysis has shown that HTGRs and their Generation IV extensions, VHTRs, have encouraging performance characteristics. Results demonstrated achievability of a singlebatch extended OTTO mode:

- There are significant differences between LEU- and TRUs-fueled VHTRs. Comparing to VHTRs with LEU, spectral transitions during operation are significantly smaller in VHTRs with TRUs. These differences affect system performance characteristics during operation.

- A single batch-mode operation appears to be potentially possible. Core lifetimes up to $7.5-9.0$ years have been observed for the TRU-fueled VHTR configurations in the present analysis.

- Fluence-related limitations (radiation damage) are the most significant constraints on achievable operation times. This constraint can be relaxed or even eliminated by reducing power density levels and using advanced radiation-tolerant materials for extended-life VHTR configurations. However, use of advanced materials will adversely impact economics characteristics.

- Acceptable safety characteristics have been observed for all configurations.

- Radiotoxicity of the TRU-fueled VHTR EOL vectors were found to require a decade-long cooling to reach radiotoxicity levels of the BOL vectors.

Thus, transitioning from LEU-systems to VHTR configurations with TRUs allows prolonging operation without 


\section{Utilization of MAs as a Fuel Component for Ultra-Long Life} VHTR Configurations: Designs, Advantages and Limitations

\section{Project 05-094}

\section{Final Scientific/Technical Report}

refueling up to almost a decade-long OTTO mode that is limited by radiation damage effects. The symbiotic quasisustainable PWR-VHTR system has been established. Its feasibility and performance characteristics will be explored further in future studies. The corresponding destruction rates are about $30 \%$. At the same time, radiotoxicity levels in nuclear waste streams require certain cooling times to drop below initial levels.

\section{ACKNOWLEDGMENTS}

This paper is based upon work supported by the U.S. Department of Energy under Award Number DE-FC0705ID14655 (05-094).

\section{DISCLAIMER}

This paper was prepared as an account of work sponsored by an agency of the United States Government. Neither the United States Government nor any agency thereof, nor any of their employees, makes any warranty, express or implied, or assumes any legal liability or responsibility for the accuracy, completeness, or usefulness of any information, apparatus, product, or process disclosed, or represents that its use would not infringe privately owned rights

Reference herein to any specific commercial product, process, or service by trade name, trademark, manufacturer, or otherwise does not necessarily constitute or imply its endorsement, recommendation, or favoring by the United States Government or any agency thereof:

The views and opinions of authors expressed herein do not necessarily state or reflect those of the United States Government or any agency thereof.

\section{REFERENCES}

1. D. E. AMES II, "Configuration Adjustment Potential of the VHTR Prismatic Cores with Advanced Actinide Fuels", Thesis, Master of Science, August 2006, Nuclear Engineering, Texas A\&M University (2006).

2. G. B. BRUNA et al., "Uncertainty Analysis and Optimization Studies on the Deep-Burner-Modular Helium Reactor (DB-MHR) for Actinide Incineration", Proc: PHYSOR2004 - The Physics of Fuel Cycle and Advanced Nuclear System: Global Developments. Chicago, USA, April 25-29, 2004, American Nuclear Society, Lagrange Park, IL. (2004).

3. "Critical Experiments and Reactor Physics Calculations for Low-Enriched HTGRs", IAEA-TECDOC-1249. Intemational Atomic Energy Agency, Viemna, Austria (2001).

4. "Evaluation of High Temperature Gas-cooled Reactor Performance; Benchmark Analysis Related to Initial Testing of the HTTR and HTR-10". IAEA-TECDOC1382, International Atomic Energy Agency, Vienna, Austria (2003).

5. "Final Environmental Impact Statement for a Geologic Repository for the Disposal of Spent Nuclear Fuel and
High-Level Radioactive Waste at Yucca Mountain, Nye County, Nevada", Vol. 2, Appendix A. "Inventory and Characteristics of Spent Nuclear Fuel, High-Level Radioactive Waste, and Other Materials", DOE/EIS-0250, U.S. DOE (2002).

6. "Generation IV Nuclear Energy Systems: Ten Year Program Plan", March 2005, Office of Advanced Nuclear Research, DOE Office of Nuclear Energy, Science, and Technology, U.S. DOE (2005)

7. T. KIM. T. TAIWO, R. IIILL, W, YANG, F. VENNERI, “A Feasibility Study of Reactor-Based Deep-Burn Concepts", ANL-AFCI-155, Argomne National Laboratory (2005).

8. K. KUNITOMI. "Development of New Type of HTGR", Proc. 73rd JSME Fall Annual Meeting, JSME, Japan, 1995.

9. T. LEWIS, D. AMES, P. TSVETKOV, "Analysis of TRUFueled VHTR Prismatic Core Performance Domains", Trans. Amer Soc, 97, 859 (2007)

10. K. MINATO, T, OGAWA, K. SAWA, A. ISHIKAWA. T. TOMITA, S. IIDA, H. SEKINO, "Irradiation Experiment on $\mathrm{ZrC}$-Coated Fuel Particles for High Temperature GasCooled Reactors", Nucl. Techn., 130, 272 (2000).

11. K. MINATO, K. SAWA, I KOYA, T. TOMITA, A. ISHIKAWA, "Fission Product Release Beliavior of Individual Coated Fuel Particles for High Temperature Gas-Cooled Reactors", Nucl. Techn., 131, 36 (2000).

12. C M. N. A PEREIRA, "Evolutionary Multicriteria Optimization in Core Design: Basic Investigations and Case Study". Annals of Nuclear Energy. 31. 1251-1264 (2004)

13. R. PLUKIENE, D. RIDIKAS, "Modeling of HTRs with Monte Carlo: from a Homogeneous to an Exact Heterogeneous Core with Microparticles", An. Nucl. En., 30, 1573, (2003)

14. "Report to Congress on Advanced Fuel Cycle Initiative: The Future Path for Advanced Spent Fuel Treatment and Transmutation Research", 03-GA50439-06, January. 2003. Office of Nuclear Energy, Science, and Technology, U.S DOE (2003).

15. C. RODRIGUEZ, A. BAXTER, D. MCEACHERN, M FIKANI, F. VENNERI, "Deep-Burn: Making Nuclear Waste Transmutation Practical", Nuol. Eng. Design, 2805 (1) (2003)

16. "SCALE: A Modular Code System for Performing Standardized Computer Analyses for Licensing Evaluation", ORNL/TM-2005/39,Ver.5.1. November 3, 2006, Oak Ridge National Laboratory, Temnessee (2006).

17. A. TALAMO, "Conceptual Design of QUADRISO Particles for High Temperature Reactors", Trans. Amer: Nucl. Soc, and Embedded Topical Meetings on Isotopes for Medicine and Industry and Nuclear Fuels and Structural Materials for the Next Generation Nuclear Reactors, 98, 727-728 (2008)

18. P. TSVETKOV, A. ALAJO, D. AMES, M. PRITCHARD, "Spectrum Shifting as a Mechanism to Improve Performance of VHTRs with Advanced Actinide Fuels", 


\section{Utilization of MAs as a Fuel Component for Ultra-Long Life} VHTR Configurations: Designs, Advantages and Limitations

Project 05-094

Final Scientific/Technical Report

Proc. 14th Intern. Conf. Nucl. Eng. (ICONE 14), Miami, Florida, July 17 - 20, 2006, ASME (2006).

19. P. V. TSVETKOV, T. G. LEWIS III, A. B. ALAJO, "Utilization of TRUs in VHTRs - Operation in a SingleBatch Mode: Front End, Back End, and Performance", Trans. Amer: Nucl. Soc. and Embedded Topical Meetings on Isotopes for Medicine and Industry and Nuclear Fuels and Structural Materials for the Next Generation Nuclear Reactors, 98, 733-735 (2008).

20. P. V. TSVETKOV, A. B. ALAJO, T, G. LEWIS III, D. E. AMES II, "Out-of-Core Fuel Cycle Characteristics of VHTRs with No On-Site Refueling", Proc. 2008 Intern. Congress on Advances in Nucl. Power Plants, Embedded Topical Meeting, June 8-12, 2008, Anaheim, California, USA, 298-302 (2008). 


\section{Conclusions and Follow-on}

Although indicating some technical limitations and challenges, studies of VHTRs with TRUs/MAs definitely suggest promising performance and possibility to utilize the core configurations with TRUs/MAs gaining prolonged operation and self-sustainability:

- There are significant differences between LEU- and TRUs-fueled VHTRs. These differences affect system performance characteristics during operation.

- A single batch-mode operation appears to be potentially possible. Core lifetimes up to 7.5 years have been observed in the present analysis.

- Fluence-related limitations (radiation damage) are the most significant constraints on achievable operation times. This constraint can be relaxed or even eliminated by reducing power density levels and using advanced radiation-tolerant materials for extended-life VHTR configurations. However, use of advanced materials will adversely impact economics characteristics.

- Acceptable safety characteristics have been observed for all configurations. However, low delayed neutron yields may result in design challenges.

Thus, the TRU-fueled VHTRs offer performance characteristics that would be difficult to achieve in analogous LEU-fueled systems - almost a decade-long batch mode operation without intermediate refueling, significant reductions of initial excess reactivity levels (smaller lifetime reactivity swings), and inherently higher achievable burnup levels.

At the same time, the results clearly illustrate that use of TRU vectors as a fuel inherently facilitates development of specially designed VHTRs with core materials withstanding performance conditions of systems optimized for fuel loading with TRUs. Limiting fast fluences lead to larger resulting radiation damage effects. There are significant core physics differences due to spectral shift effects towards harder neutron spectra with substantially reduced neutron populations at thermal energies.

The research work advanced according to the 3-years plan and has been completed in full. No scope-related changes were made from the DOE approved application. The project accomplishments are fully consistent with the project goals/objectives. The project results have been extensively reported at the ANS and ASME conferences as well as have been published in archival peer-reviewed journals. 


\title{
Utilization of MAs as a Fuel Component for Ultra-Long Life
} VHTR Configurations: Designs, Advantages and Limitations

LWR-VHTR Fuel Cycles with Dedicated Small 14MeV Waste Incineration Back-End Clean-up Facilities

\author{
Pavel V. Tsvelkov
}

Department of Nuclear Engineering, Texas -4 \& M Universily: 129 Zachry Engineering Cenler, MS 3133 TAMU Callege Station, 2 X, 77843-3133, E-mail:tsverkovâumu ediu

\section{IVTRODUCTION}

Thermal reactors. IWRs and later VHTRs. are expected to dominate the $21^{\text {th }}$ century nuclear power options. $\lfloor 1,2\rfloor$ As a result, near-lenm deployable waste management and miminuzation options have to rely on incineration strategies involving thermal spectrum systems, for example. very efficient deep burn strategies. or potential biuary configurations imvolving thermal spectrum deep-lsum systems angmented by small dedieated fast spectrum facilities. [3] Minimization or elimination (cleanup) of high level waste (HLW) inventories (transuranics (TRUs) and long-lived fission products (FP)) is an essential component of publiclyacceptable sustainable nuclear energy strategies. [1]

In the present preliminary analysis, the near-tem deployable dedicated small $14 \mathrm{MeV}$-neutron waste incineration back-end clean-up facilities are envisioned as advanced incinerators that are used in combination with themnal reactors to minimize the remaining long-lived radioactive species. These $14 \mathrm{MeV}$ transmuters are viewed as small facilities designed to reduce waste inventories prior to reprocessing stages minimizing spent fuel activity levels and repository requirements. The focus is on the back-end fuel cycle chatacteristics and system performance, especially examining envirommentally benign fuel cycle options. The preliminary study is performed within the framework of the U.S. DOE NERI project that targets design envelops of TRU- VHTRs.[4]

\section{FUEL CYCLE CONCEPT}

Figure 1 shows the envisioned LWR-VHTR fuel cycle with TRU-recycle and dedicated waste mimmization facilities that is optimized to yield substantially reduced waste inventories for disposal in the geological repository. In this scheme, PWR fuel elements with $3.75 \%$ LEU. which were burnt up to 41,200 $\mathrm{MWd} / \mathrm{mtHM}$ and cooled for up to 23 years, are being reprocessed.[5] Partitioned TRU and depleted uranium (DU) are then recycled in VHTRs (continuous lines) and potentially in PWRs (dashed line). It is assumed that both. LWR and VHTR, spent nuclear fuel streams will go through the dedicated waste minimization facilities before entering the reprocessing stages. When fast reactors (FR) become viable, the suggested fiuel cycle scheme may be transformed into a sustainable nuclear energy system that does not require a LEU-feed and offers environmentally benign performance characteristics.

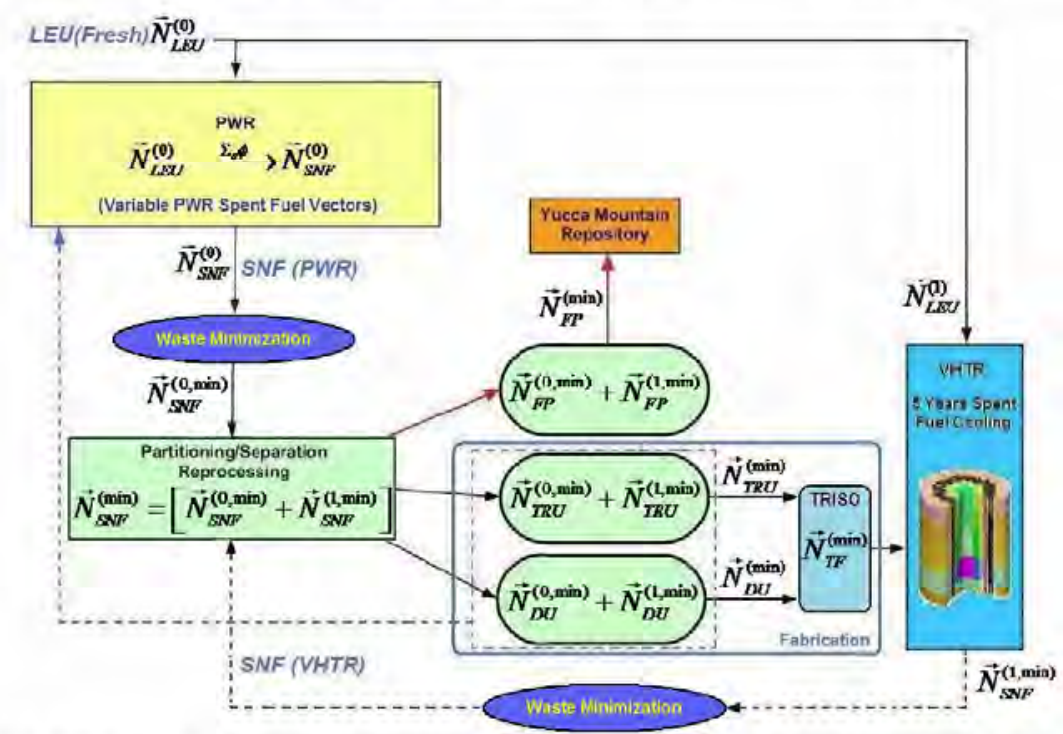

Fig. 1. Advanced fuel cycles with LWRs, VHTRs and $14 \mathrm{MeV}$ waste incineration back-end clean-up facilities.

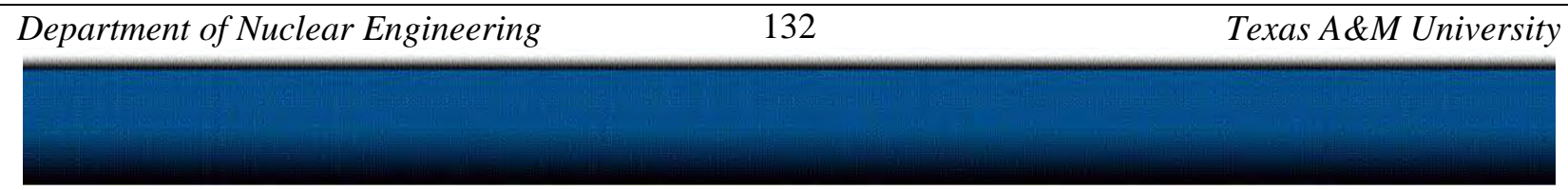




\section{Utilization of MAs as a Fuel Component for Ultra-Long Life} VHTR Configurations: Designs, Advantages and Limitations

\section{HIGH-FIDELITY REACTOR MODEL}

Detailed high-fidelity exact-geometry models were developed for representative reactor configurations. They were used in preliminary calculations with the ORNL SCALE 5.1 code system.[6] The reactor models have been benchmarked against existing experimental data and reactor design data.[7]

\section{PRELIMINARY RESULTS}

Results of initial evaluations for advanced fuel cycle scenarios with LWR TRU-fueled VHTRs operating in a single batch mode augmented by $14 \mathrm{MeV}$ neutron incineration are shown in Table I. For the TRU-fueled VHTR spent fuel, it will take approximately 10 years of cooling time to reach the BOL activity level.[4,8,9] However, addition of $14 \mathrm{MeV}$ neutron incineration is anticipated to reduce this time to $4-5$ years. The provided estimates assume the LWR-VHTR fuel cycle scenario as shown in Fig. 1 with external $14 \mathrm{MeV}$ neutron irradiation of VHTR spent fuel composition.

TABLE I. TRU-fueled VHTRs Augmented by $14 \mathrm{MeV}$ Neutron Incineration

\begin{tabular}{|l|l|l|}
\hline \multirow{2}{*}{\begin{tabular}{l} 
Waste Minimization \\
\cline { 2 - 3 }
\end{tabular}} & \multicolumn{2}{|c|}{ Advanced Fuel Cycle } \\
\hline $\begin{array}{l}\text { Time to reach same } \\
\text { volumetric energy release } \\
\text { (years)* }\end{array}$ & 71 & TRU-VHTR+14MeV \\
\hline $\begin{array}{l}\text { Time to reach same } \\
\text { specific energy release } \\
\text { (years)** }\end{array}$ & 164 & 65 \\
\hline $\begin{array}{l}\text { Time to reach same } \\
\text { volumetric activity level } \\
\text { (years)* }\end{array}$ & 10 & 4.3 \\
\hline $\begin{array}{l}\text { Time to reach same } \\
\text { specific activity level } \\
\text { (years)** }\end{array}$ & 23 & 9.0 \\
\hline
\end{tabular}

*per unit volume

**per unit mass

\section{CONCLUSIONS}

The preliminary analysis has shown promising advanced fuel cycle scenarios that include LWRs, VHTRs and dedicated $14 \mathrm{MeV}$-neutron waste incineration facilities. If deployed, these scenarios may substantially reduce nuclear waste inventories approaching environmentally benign nuclear energy system characteristics. Computational approaches, analysis metrics, and benchmark strategies have been established for future detailed studies.

\section{ACKNOWLEDGMENTS}

This paper is based upon work supported by the U.S. Department of Energy under Award Number DE-FC0705ID14655 (05-094).

\section{REFERENCES}

1. R. BROGLI, R. KRAKOWSKI, "Degree of Sustainability of Various Nuclear Fuel Cycles," LAUR-01-6939, ISSN 1019-0643, Nuclear Energy and Safety Research Department, Paul Scherrer Institute (2002).

2. "Report to Congress on Advanced Fuel Cycle Initiative: The Future Path for Advanced Spent Fuel Treatment and Transmutation Research", 03GA50439-06, U.S. DOE (2003).

3. T. KIM, T. TAIWO, R. HILL, W. YANG, F. VENNERI, "A Feasibility Study of Reactor-Based Deep-Burn Concepts", ANL-AFCI-155, Argonne National Laboratory (2005).

4. T. LEWIS, D. AMES, P. TSVETKOV, "Analysis of TRU-Fueled VHTR Prismatic Core Performance Domains", Trans. Amer. Soc., 97, 859 (2007).

5. "Final Environmental Impact Statement for a Geologic Repository for the Disposal of Spent Nuclear Fuel and High-Level Radioactive Waste at Yucca Mountain, Nye County, Nevada", 2, A, "Inventory and Characteristics of Spent Nuclear Fuel, High-Level Radioactive Waste, and Other Materials," DOE/EIS-0250, U.S. DOE (2002).

6. "SCALE: A Modular Code System for Performing Standardized Computer Analyses for Licensing Evaluation", ORNL/TM-2005/39,Ver.5.1, November 3, 2006, Oak Ridge National Laboratory, Tennessee (2006).

7. D. E. AMES, II, P. V. TSVETKOV, "Benchmark Efforts to Support Studies of VHTRs with TRUs", Proc. $4^{\text {th }}$ Intern. Meeting on High Temperature Reactor Technology (HTR 2008), Washington, D.C., September 28-October 1, 2008 (to be published).

8. P. V. TSVETKOV, T. G. LEWIS III, A. B. ALAJO, "TRU-Fueled VHTRs for Applications Requiring an Extended Operation with Minimized Control and No Refueling", ICONE16-48334, Proc. $16^{\text {th }}$ Intern. Conf. Nucl. Eng. (ICONE 16), Orlando, Florida, May 11-15, 2008, ASME (2008).

9. P. V. TSVETKOV, A. B. ALAJO, T. G. LEWIS III, D. E. AMES II, "Out-of-Core Fuel Cycle Characteristics of VHTRs with No On-Site Refueling", Proc. 2008 Intern. Congress on Adv. In Nucl. Power Plants (ICAPP 2008), Anaheim, California, June 8-12, 2008, ICAPP08-8233, ANS, pp. 298-302 (2008).

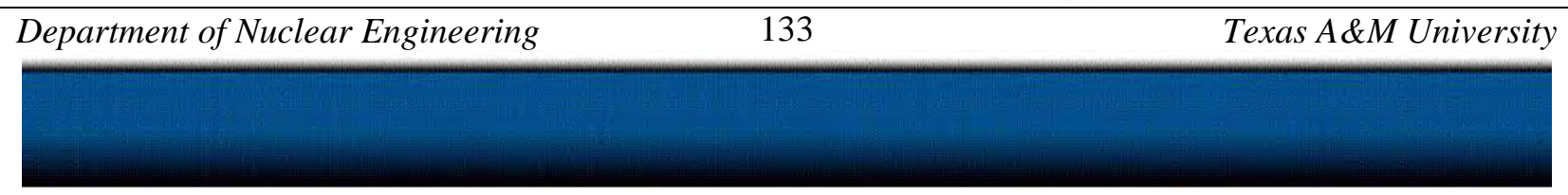




\section{BIBLIOGRAPHY}

1. F. Venneri, et al, "Deep Burn Transmutation: A Practical Approach to the Destruction of Nuclear Waste in the Context of Nuclear Power Sustainability," General Atomics, FDOE00- N-TRT-X-000132, Dec. 19, 2001

2. $\quad$ P. Peterson, Science and Global Security, Vol 6, No 1., pp 1-29 (1996).

3. Potter, and A. Shenoy, "Gas Turbine-Modular Helium Reactor (GTMHR) Conceptual Design Description Report,” GA Report 910720, Revision 1, General Atomics, July 1996.

4. J. A. Stillman, "Homogeneous Recycling Strategies in LWRs for Plutonium, Neptunium, and Americium Management,” Argonne National Laboratory AFCI Report, ANL-AFCI124, August 31, 2004.

5. T. K. Kim, W. S. Yang, T. A. Taiwo, and H. S. Khalil, "Whole-Core Depletion Studied in Support of Fuel Specification for the Next Generation Nuclear Plant (NGNP)," Argonne National Laboratory Gen IV Report, July 30, 2004.

6. T. A. Taiwo, W. S. Yang, M. A. Smith, T. K. Kim, and H. S. Khalil, “Assessment of Reactor Physics Codes for Analysis and Design of the Very High Temperature Reactor (VHTR),” Argonne National Laboratory Gen IV Report, September 30, 2004.

7. T. K. Kim, W. S. Yang, M. A. Smith, T. A. Taiwo, and H. S. Khalil, “Assessment of Monte Carlo and Deterministic Codes for Next Generation Nuclear Plant (NGNP) Core Modeling,” Argonne National Laboratory Gen IV Report, April 15, 2004.

8. "WIMS - A Modular Scheme for Neutronics Calculations,” User's Guide for Version 8, ANSWER/WIMS(99)9, The ANSWERS Software Package, AEA Technology.

9. G. Marleau, et al, “A User Guide for DRAGON,” Technical report IGE-174 Rev. 4, Ecole Polytechnique de Montréal, September 1998 (1998).

10. “MCNP - A General Monte Carlo N-Particle Transport Code,” Version 4C, Los Alamos National Laboratory, LA-13709-M (1993).

11. B. J. Toppel, “A User's Guide to the REBUS-3 Fuel Cycle Analysis Capability,” ANL83-2, Argonne National Laboratory (1983).

12. T. D. Burchell, "Fission Reactor Applications of Carbon,” In: Carbon Materials for Advanced Technologies, T. D. Burchell (Editor). Chapter 13, pp. 429-484, Pub. Elsevier Science Ltd, 1999. 
Utilization of MAs as a Fuel Component for Ultra-Long Life VHTR Configurations: Designs, Advantages and Limitations

Project 05-094

Final Scientific/Technical Report

13. E. E. Bende, A. H. Hogenbirk, J. L. Kloosterman, and H. Van Dam, "Analytical Calculations of the Average Dancoff Factor for a Fuel Kernel in a Pebble Bed HighTemperature Reactor,” Nuclear Science and Engineering, 133, 147 (1999).

14. Z. Karriem, C. Stoker, and F. Reitsma, "MCNP Modeling of HTGR Pebble-Type Fuel," Proc. of Monte Carlo 2000 Conference, Lisbon, Portugal, October 23-26, 2000 (2000).

15. R.A. Simon and P.D. Capp, "Operating Experience with the DRAGON High Temperature Reactor Experiment," HTR-2002, Proceedings of the Conference on High Temperature Reactors, Petten, NL, April 22-24, 2002, Reproduced by the IAEA Vienna, Austria, 2002.

16. J. P. H. Blake, V.E. Della Loggia, J. Reber, "Physics Experiments on the Dragon Reactor Experiment,” D. P. Report 166, May 1963. (Obtained from CD containing OECD Dragon Project Reports, NEA-1726/01.)

17. R. Cameron et al., "Measurements of Control Rod Worth and Excess Reactivity on the First Core of Dragon”, D. P. Report 359, July 1965. (Obtained from CD containing OECD Dragon Project Reports, NEA-1726/01.)

18. V. E. Della Loggia et al., "Zero Energy Experiments on the Dragon Reactor Prior to Charge IV Startup”, D. P. Report 820, Jan. 1973. (Obtained from CD containing OECD Dragon Project Reports, NEA-1726/01.)

19. DRAGON Project \& its Contribution to the Development of the High Temperature Reactor (HTR),” D. P. Report 1000, November 1978. (Obtained from CD containing OECD Dragon Project Reports, NEA-1726/01.)

20. H. Yasuda, et al., "VHTRC Temperature Coefficient Benchmark Problem,” JAERIData/ Code 94-103, Japan Atomic Energy Research Institute, October 1994.

21. "Critical Experiments and Reactor Physics Calculations for Low-Enriched High Temperature Gas-Cooled Reactors,” IAEA TECDOC 1249, Vienna, 2001.

22. J. R. Brown et al., "Physics Testing at Fort St. Vrain - A Review,” Nucl. Sci. Eng., 97, 104 (1987).

23. R. G. Bardes, et al., "Results of HTGR Critical Experiments Designed to Make Integral Checks on the Cross Sections in Use at Gulf General Atomic," GA-8468, GA Technologies, Inc., (Feb. 1968).

24. V.E. Della-Loggia, T.S. Playle, "Reactor Physics Measurements on the Zero Energy HTR Lattice HITREX-1 at CEGB Berkeley Nuclear Laboratories,” D. P. Report 925, March 1975. (Obtained from CD containing OECD Dragon Project Reports, NEA1726/01.) 
Utilization of MAs as a Fuel Component for Ultra-Long Life VHTR Configurations: Designs, Advantages and Limitations

Project 05-094

Final Scientific/Technical Report

25. D. F. Newman, “Temperature-Dependent kळ for a ThO2-PuO2 HTGR Lattice,” Nucl. Technol., 19, 66 (1973).

26. "Evaluation of High Temperature Gas Cooled Reactor Performance: Benchmark Analysis Related to the HTTR and HTR-10,” IAEA TECDOC-1382, Vienna, 2003.

27. M. Brunet, "Marius IV: A Critical Experiments Programme on GGA Type Lattices," DCPM 19/CEA-2 (obtained from OECD/NEA dataset on DRAGON Project Reports, from December 2003 IRhPE Meeting).

28. C. A. Preskitt et al., "Interpretation of Pulsed-Source Experiments in the Peach Bottom HTGR,” Nucl. Sci. Eng., 29, 283 (1967). 2. “Critical Experiments and Reactor Physics Calculations for Low-Enriched High Temperature Gas-Cooled Reactors," IAEA TECDOC-1249, Vienna, 2001.

29. R. G. Bardes et al., "High Temperature Gas Cooled Reactor Critical Experiment and Its Application,” Proc. IAEA Symp. Exponential and Critical Experiments, Amsterdam, Netherlands, September 2-6, 1963, conference paper SM-42/37, International Atomic Energy Agency (1963).

30. Y. Kaneko, "Reactor Physics Research Activities Related to the Very High Temperature Reactor in Japan,” Nucl. Sci. Eng. 97, 145 (1987).

31. Johnstone, V.E. Della Loggia, "Experimental Results from the UKAEA Reactor Physics Programme on Low Enrichment HTR Lattices at AEE, Winfrith,” D. P. Report 730, August 1970. (Obtained from CD containing OECD Dragon Project Reports, NEA1726/01.)

32. R. Bratton, NGNP Graphite Selection Strategy, INL/EXT-05-00269 (May 2005).

33. W. Windes, T. Burchell, and R. Bratton, NGNP Graphite Technology Development Plan, Idaho National Laboratory (2007).

34. T. Burchell, Carbon Materials for Advanced Technologies, Chap. 13, "Fission Reactor Applications,” Elsevier Science (1999).

35. T. Burchell, et al., Next-Generation Nuclear Plant Phenomena Identification and Ranking Tables (PIRTs)_Volume 5: Graphite PIRTs (NUREG/CR-6944, Vol. 5), ORNL/ TM2007/147, Vol. 5, Oak Ridge National Laboratory.

36. Presentation by Carbon Lorraine to General Atomics, “Carbone Graphite Grades” (2007).

37. ASTM D7219-05, "Standard Specification for Isotropic and Near-Isotropic Nuclear Graphites,” ASTM International, U.S.A. (2005). 
38. A Technology Roadmap for Generation IV Nuclear Energy Systems, GIF-002-00, Generation IV International Form, (Dec. 2002).

39. Toward a More Secure and Cleaner Energy Future for America, National Hydrogen Energy Roadmap, U.S. Department of Energy, November 2002.

40. P. E. MacDonald et al. NGNP preliminary Point Design - Results of the Initial Neutronics and Thermal-Hydraulic Assessments, INEEL/EXT-03-00870, July 2003.

41. General Atomics, Gas Turbine-Modular Helium Reactor (GT-MHR) Conceptual Design Description Report, 910720, revision 1, July 1996.

42. "Program and Project Management Policy for the Acquisition of Capital Assets", DOE O 413.1, October 13, 2000

43. Project Management for the Acquisition of Capital Assets, DOE M 413.3-1, U.S. DOE Office of Management, Budget and Evaluation, June 28, 2003.

44. Advanced Gas Reactor Team, Technical Program Plan for the Advanced Gas Reactor Fuel Development and Qualification Program, ORNL/TM-2002/262, April 2003.

45. D. Petti, J. Buongiorno, J. Maki, R. Hobbins, G. Miller, "Key Differences in the Fabrication, Irradiation and High-Temperature Accident Testing of U.S. and German TRISO-coated Particle Fuel, and Their Implications on Fuel Performance”, Nuclear Engineering and Design, Vol. 222, pp. 281-297, 2003.

46. IAEA, Hydrogen as an Energy Carrier and its Production by Nuclear Power, IAEATECDOC- 1085, May 1999.

47. L. C. Brown et al., High Efficiency Generation of Hydrogen Fuels Using Nuclear Power, Final Technical Report for the Period August 1, 1999 Through September 30, 2002, GAA24285, June 2003.

48. P. E. MACDONALD et al., "NGNP Preliminary Point Design - Results of Initial Neutronics and Thermal-Hydraulic Assessment," INEEL/EXT-03-00870 Rev. 1, Idaho National Engineering and Environmental Laboratory, September 2003.

49. G. ALIBERTI, G. PALMIOTTI, M. SALVATORES, C. G. STENBERG, “Transmutation Dedicated Systems: An assessment of Nuclear Data Uncertainty Impact," Nucl. Sci. and Eng. 146, 13-50, (2004).

50. G. PALMIOTTI, G. ALIBERTI, M. SALVATORES, J. TOMMASI, “Integral Experiment Analysis for Validation and Improvement of Minor Actinide Data for Transmutation Needs,” Proc. Int. Conf. ND-2004, Santa Fe, Sept. 2004. 
Utilization of MAs as a Fuel Component for Ultra-Long Life VHTR Configurations: Designs, Advantages and Limitations

Project 05-094

Final Scientific/Technical Report

51. G. Aliberti, G. Palmiotti, M. Salvatores, "Methods in Use for Sensitivity Analysis, Uncertainty Evaluation, and Target Accuracy Assessment”. INL/CON-07-13356. October (2007).

52. G. PALMIOTTI, M. SALVATORES, "Proposal for Nuclear Data Covariance Matrix,” JEFDOC 1063 Rev.1, January 2005.

53. KODELI, E. SARTORI, ZZ-COV-15GROUP-2005, NEA-1730 Package, OECD/NEA Data Bank (in preparation).

54. "Uncertainty Analysis”, Y. RONEN Editor, CRC Press 1988 and article by J.H. MARABLE et al. in " Advances in Nuclear Science and Technology,” Vol. 14, J. LEWINS and A. BECKER Editors, Plenum Publishing Corporation, 1982.

55. See e.g. article by A. GANDINI in "Uncertainty Analysis”, Y. RONEN Editor, CRC Press 1988 and article by E. GREENSPAN in " Advances in Nuclear Science and Technology,” Vol. 14, J. LEWINS and A. BECKER Editors, Plenum Publishing Corporation, 1982.

56. L. N. USACHEV and Y. BOBKOV, "Planning on Optimum Set of Microscopic Experiments and Evaluations to Obtain a Given Accuracy in Reactor Parameter Calculations,” INDC CCP-19U, IAEA Int. Nucl. Data Committee, 1972.

57. GANDINI, G. PALMIOTTI, M. SALVATORES, “Equivalent Generalized Perturbation Theory (EGPT),” Ann. Nucl. Energy, Vol. 13, n.3, pp. 109-114, 1986.

58. J. M. KALlFELZ, G. BRUNA, G. PALMIOTTI, M. SALVATORES, "Burn-up Calculations with Time-Dependent Generalized Perturbation Theory,” Nucl. Sci. Eng. 62, 304 (1977).

59. M. L. WILLIAMS, "Development of Time-Dependent Perturbation Theory for Coupled Neutron/Nuclide Fields,” Nucl. Sci. Eng. 70, 20-36 (1979).

60. J. R. WHITE, "The DEPTH-CHARGE Static and Time-Dependent Perturbation/Sensitivity System for Nuclear Reactor Core Analysis,” Oak Ridge National Laboratory, ORNL/CSD- 78, (1981).

61. G. PALMIOTTI, R. F. BURSTALL, E. KIEFHABER, W. GEBHARDT, J. M. RIEUNIER, "New Methods Developments and Rationalization of Tools for LMFBR Design in the Frame of the European Collaboration,” FR'91 International Conference on Fast Reactors and Related Fuel Cycles, Kyoto, Japan, October 28 - November 1,1991.

62. W. S. YANG, T. J. DOWNAR, "Depletion Perturbation Theory for the Constrained Equilibrium Cycle,” Nucl. Sci. Eng., 102, 365 (1989).

63. C. H. ADAMS, Private Communications, 2005. 
Utilization of MAs as a Fuel Component for Ultra-Long Life VHTR Configurations: Designs, Advantages and Limitations

Project 05-094

Final Scientific/Technical Report

64. G. PALMIOTTI, J.M. RIEUNIER, C. GHO, M. SALVATORES, "BISTRO Optimized Two Dimensional Sn Transport Code,” Nucl. Sci. Eng. 104, 26 (1990)

65. M. J. BELL, “The ORNL Isotope Generation and Depletion Code,” Oak Ridge National Laboratory, May 1973.

66. "WIMS - A Modular Scheme for Neutronics Calculations, User's Guide for Version 8," ANSWER/WIMS(99)9, The ANSWERS Software Package, AEA Technology.

67. T. K. KIM, W. S. YANG, T. A. TAIWO, H. S. KHALIL, "Equilibrium Cycle Analysis in Support of Fuel Specification for Next Generation Nuclear Power Plant,” Proc. of ICAPP ’05, Seoul, Korea, May 15-19, 2005.

68. R. C. POTTER et al., "Gas Turbine-Modular Helium Reactor (GT-MHR) Conceptual Design Description Report,” GA Report 910720, Revision 1, General Atomics, July 1996.

69. G. PALMIOTTI, M. SALVATORES, R.N. HILL, "Sensitivity, Uncertainty Assessment, and Target Accuracies Related to Radiotoxicity Evaluation”, Nucl. Sci. Eng. 117, 239250 (1994).

70. DEPARTMENT OF ENERGY, Report to Congress Advanced Fuel Cycle Initiative: Objectives, Approach, and Technology Summary (2005).

71. DEPARTMENT OF ENERGY, Advanced Fuel Cycle Initiative Comparison Report, FY 2006 Update (2006).

72. DEPARTMENT OF ENERGY, Advanced Fuel Cycle Initiative Comparison Report, FY 2007 Update, in final preparation.

73. W. G. HALSEY, Advanced Fuel Cycle Initiative (AFCI) Repository Impact Evaluation FY-05 Progress Report, UCRL-TR-215380 (2005).

74. W. S. YANG, Y. KIM, R. N. HILL, T. A. TAIWO, H. S. KHALIL, "Long-Lived Fission Product Transmutation Studies,” Nuclear Science and Engineering, 146, pages 291-318 (2004).

75. R. A. WIGELAND, T. H. BAUER, T. H. FANNING, and E. E. MORRIS, "Separations and Transmutation Criteria to improve Utilization of a Geologic Repository,” Nuclear Technology, 154 (2006).

76. PR\&PP WORKING GROUP, Evaluation Methodology for Proliferation Resistance and Physical Protection of Generation IV Nuclear Energy Systems, Generation IV International Forum, GIF/PRPPWG/2006/005 (2006). 


\section{Utilization of MAs as a Fuel Component for Ultra-Long Life} VHTR Configurations: Designs, Advantages and Limitations

77. $\quad$ E. A. HOFFMAN, W. S. YANG, R. N. HILL, Preliminary Core Design Studies for the Advanced Burner Reactor over a Wide Range of Conversion Ratios, ANL-AFCI-177 (2006).

78. E. A. HOFFMAN, Updated Design Studies for the Advanced Burner Reactor over a Wide Range of Conversion Ratios, ANL-AFCI-189 (2007).

79. M. ASGARI, B. FORGET, S. PIET, R. FERRER, S. BAYS, Computational Neutronics Methods and Transmutation Performance Analyses for Light Water Reactors, INL/EXT07-12472 (2007).

80. S. J. PIET, G. E. MATTHERN, J. J. JACOBSON, C. T. LAWS, L. C. CADWALLADER, A. M. YACOUT, R. N. HILL, J. D. SMITH, A. S. GOLDMANN, G. BAILEY, Fuel Cycle Scenario Definition, Evaluation, and Trade-offs, INL/EXT-0611683 (2006).

81. NGNP Point Design - "Results of the Initial Neutronics and Thermal-Hydraulic Assessments During FY-03,” Idaho National Engineering and Environmental Laboratory, INEEL/EXT-03-00870 Rev. 1, September 2003.

82. G. S. Chang and J. M. Ryskamp, "Depletion Analysis of Mixed Oxide Fuel Pins in Light Water Reactors and the Advanced Test Reactor,” Nucl. Technol., 129, No. 3, pp. 326-337 (2000).

83. J. F. Briesmeister (Editor), "MCNP-A General Monte Carlo N-Particle Transport Code, Version 4C,” LA-13709-M, Los Alamos National Laboratory (2000).

A. G. Croff, "ORIGEN-2: A Versatile Computer Code for Calculating the Nuclide Compositions and Characteristics of Nuclear Materials,” Nucl. Technol., 62, pp. 335-352 (1983).

84. G. S. Chang, "VHTR Double-Heterogeneous and Homogenous Lattice Model Burnup Characteristics Comparison," Trans. Am. Nucl. Soc., Vol. 90, 37-39 (2004).

85. National Academy of Sciences Committee on International Security and Arms, Management and Disposition of Excess Weapons Plutonium, National Academy Press, 1994.

86. Wigeland, R., et. al. "Repository Impact of LWR MOX and Fast Reactor Recycling Options”. ANS Topical Meeting. New Orleans, LA 2003.

87. Yacout, A., et. al. "Dynamic Analysis of the AFCI Scenarios". Proceedings of the PHYSOR 2004: The Physics of Fuel Cycles and Advanced Nuclear Systems - Global Developments 2004: 785-794 
Utilization of MAs as a Fuel Component for Ultra-Long Life VHTR Configurations: Designs, Advantages and Limitations

Project 05-094

Final Scientific/Technical Report

88. Bathke, C., et. al. "Report of LANL Advanced Fuel Cycle Systems Analyses for FY 2003”. Los Alamos National Laboratory. 2004.

89. J. Saling. Radioactive Waste Management, 2nd Edition. Taylor and Francis, 2001.

90. Bunn, M., et. al. "Economics of Reprocessing vs. Direct Disposal of Spent Nuclear Fuel”. Harvard University, 2003

91. Rose, P. F. and C. L. Dunford. "ENDF-102: Data Formats and Procedures for the Evaluated Nuclear Data File ENDF-6”. Brookhaven National Laboratory. 1990.

92. Turinsky, P., H. Abdel-Khalik, and T.E. Stover. "Development of Uncertainty Analysis Capabilities for the SINEMA Project, FY 2006 Report”. North Carolina State University. 2006.

93. Duderstadt, J., and L. Hamilton. Nuclear Reactor Analysis. Hoboken, NJ. John Wiley \& Sons, Inc., 1976.

94. Schneider, E., and C. Bathke, "Sensitivity of Fuel Cycle Performance Metrics to Perturbations in Cross-Sections”. Transactions of the American Nuclear Society v 92, 2005.

95. Ronen, Yigal. Uncertainty Analysis. Boca Raton, FL. CRC Press Inc., 1988.

96. Aliberti, G., et. al. "Nuclear data sensitivity, uncertainty and target accuracy assessment for future nuclear systems”. Annals of Nuclear Energy, 33(8), 2006: 700-733.

97. Aliberti, G., et al. "Impact of Nuclear Data Uncertainties on Transmutation of Actinides in Accelerator-Driven Assemblies,” Nuclear Science and Engineering v 146, 2004: 1350 .

98. H. Abdel-Khalik, “Adaptive Core Simulation”. Ph.D. Dissertation. North Carolina State University, 2004.

99. Cacuci, Dan. Sensitivity and Uncertainty Analysis, Volume I. Chapman \& Hall/CRC Press, 2005.

100. Olsson, A., and G. Sandberg, "Latin Hypercube Sampling for Stochastic Finite Element Analysis”. Journal of Engineering Mechanics January 2002.

101. Bendat, J., and A. Piersol. Random Data: Analysis and Measurement Procedures. 2nd Ed. Hoboken, NJ. John Wiley \& Sons, Inc., 1986.

102. Kawano, T., et. al. "Evaluations and Propagation of the Pu-239 Fission Cross-Section Uncertainties Using a Monte Carlo Techni1que”. Nuclear Science and Engineering v153, n 1, May 2006: 1-7. 
103. Tarantola, A., Inverse Problem Theory: Methods for Data Fitting and Model Parameter Estimation. Elsevier Science Publishers B. V., 1987.

104. Figliola, F., and D. Beasley. Theory and Design for Mechanical Measurements. 3rd Ed. Hoboken, NJ. John Wiley \& Sons, Inc., 2000.

105. Tong, Y.L. Multivariate Normal Distribution. New York, NY. Springer-Verlag, 1990.

106. Jordan, W., et. al. "SCALE Cross-Section Libraries". Oak Ridge National Laboratory. ORNL/TM-2005/39 Version 5 Vol. III, Sect. M4, 2005.

107. Rearden, B. "Sensitivity Utility Modules”. Oak Ridge National Laboratory. ORNL/TM2005/39 Version 5 Vol. III, Sect. M18, 2005.

108. Hermann, O., and M. DeHart. "Validation of SCALE (SAS2H) Isotopic Predictions for BWR Spent Fuel”. Oak Ridge National Laboratory. ORNL/TM-13315, 1998.

109. T. K. Kim, T. A. Taiwo, R. N. Hill, and J. A. Stillman, "Spent Nuclear Fuel Characterization for a VHTR”, Argonne National Laboratory, Proceedings of GLOBAL 2005, Tsukuba, Japan, Oct 9-13, 2005.

110. Emory D. Collins and John-Paul Renier, "Systems Studies of Actinide PartitioningTransmutation Recycle Methods”, Oak Ridge National Laboratory, Proceedings of GLOBAL 2005, Tsukuba, Japan, Oct 9-13, 2005.

111. G. D. Del Cul, B.B. Spencer, C.W. Forsberg, E.D. Collins, and W.S. Rickman, “TRISO Coated Fuel Processing to Support High-Temperature Gas-Cooled Reactors", ORNL/TM-2002/156, Oak Ridge National Laboratory, September, 2002.

112. "Workshop on Deep-Burn Destruction of Nuclear Waste using MHR Technology", General Atomics, November 5, 2004 San Diego, CA.

113. “Advanced Gas Reactor Fuel Development and Qualification Program”, presented at the Advanced Gas Reactor Technology Course, ANS Summer Meting, San Diego, June 10, 2005 (John Saurwein, General Atomics)

114. G. L. Bell, et al, "Technical Program Plan for the Advanced Gas Reactor Fuel Development and Qualification Program”, Oak Ridge National Laboratory Report ORNL/TM-2002/262, April 2003.

115. D. A. Petti, et al, "Technical Program Plan for the Advanced Gas Reactor Fuel Development and Qualification Program”, Idaho National Laboratory Report INL/EXT05-00465, Revision 1, August 2005.

116. J. T. Maki, “AGR-1 Irradiation Experiment Test Plan”, Idaho National Laboratory Report INL/EXT-05-00593, August 2005. 
Utilization of MAs as a Fuel Component for Ultra-Long Life VHTR Configurations: Designs, Advantages and Limitations

Project 05-094

Final Scientific/Technical Report

117. J. L. Rempe, et al, "Evaluation of Specialized Thermocouples for High-Temperature InPile Testing”, Proceedings of the International Congress on Advances in Nuclear Power Plants, Paper 6068, Reno, NV, USA, June 4-8, 2006.

118. A Technology Roadmap for Generation IV Nuclear Energy Systems, U.S. DOE Nuclear Energy Research Advisory Committtee and the Generation IV International Forum, December (2002).

119. System Design Report, Development of Generation IV Advanced Gas-Cooled Reactors with Hardened/Fast Neutron Sectrum, INERI Report GFR023, February (2005).

120. L.L. Snead, J.W. Klett, "Ceramic Composites for Structural Applications," Proc. GLOBAL 2003, American Nuclear Society (2003) 1077-1078.

121. E.A. Hofman, T.A. Taiwo, "Physics Studies of Preliminary Gas-Cooled Fast Reactor Designs,” Proc. GLOBAL 2003, American Nuclear Society (2003) 82-91.

122. H. Kishimoto, T. Hinoki, K. Ozawa, K.-H. Park, S. Kondo, A. Kohyama, "Dimension Stability Analysis of NITE SiC/SiC Composite Using Ion Bombardments for the Investigation of Reliability as Fusion Materials,” Ceramic Engineering and Science Proceedings, eds. M. Brito, P. Filip, C. Lewisohn, A. Sayir, M. Opeka, W. Mullins, vol. 26, issue 8 (2005) p. 215-222.

123. Gamma Engineering Proposal to DOE for FY-2003 SBIR Phase 1 Solicitation, "Development of a Hybrid SiC/SiC Ceramic Composite for Gas Cooled Fast Reactor Fuel Cladding and Core Structurals,” January, 2003.

124. S.J. Zinkle, “Nonfissile Ceramics for Future Nuclear Systems,” Proc. GLOBAL 2003, American Nuclear Society (2003) 1066-1067.

125. D.L. Keller, G.W. Cunningham, W.E. Murr, E.O. Fromm, D.E. Lozier, "HighTemperature Irradiation Test of UO2 Cermet Fuels,” report BMI-1608 (1963).

126. W.C. Thurber, F.R. McQuilkin, E.L. Long, M.F. Osborne, "Irradiation Testing of Fuel for Core B of the Enrico Fermi Fast Breeder Reactor,” report ORNL-3709 (1964).

127. Neeft, E.A.C., Bakker, K., Schram, R.P.C., Conrad, R., Konings, R.J.M., “The EFTTRAT3 Irradiation Experiment on Inert Matrix Fuels,” J. Nucl. Mater. 320 (2003) 106-116.

128. Georgenthum, V., Chauvin, N., Pelletier, M., Noitor, J., Berlanga, C., Brenet, D., "Influence of the Microstructure for Inert Matrix Fuel Behaviou: Experimental Results and Calculations,” Proceedings of the GLOBAL 99 International Conference on Future Nuclear Systems, Jackson Hole, WY USA August 29-September 3, (1999).

129. Nitani, N., et al., “In-pile irradiation of rock-like oxide fuels”, J. Nucl. Mater. 319 (2003) 102. 
Utilization of MAs as a Fuel Component for Ultra-Long Life VHTR Configurations: Designs, Advantages and Limitations

Project 05-094

Final Scientific/Technical Report

130. Noirot, J., Desgranges, L., Chauvin, N., Georgenthum, V., "Postirradiation Examinations of THERMHET Conmposite Fuels for Transmutation,” J. Nucl. Mater. 320 (2003) 117125.

131. R.A. Lowden, J.C. McLaughlin, J.R. Kelley, "Nitride Ceramic Coatings for Gas-Cooled Fast Reactor Particle Fuel,” Proc. GLOBAL 2003, American Nuclear Society (2003) 523-524.

132. Minato, K., Ogawa, T., Fukuda, K., Sekino, H., Kitagawa, I., Mita, Naoaki, "Fission Product Release from ZrC-coated Fuel Particles During Postirradiation Heating at 1800 and $2000^{\circ} \mathrm{C}$,” J. Nucl. Mater. 249 (1997) 142-149.

133. S.J. Zinkle, "Nonfissile Ceramics for Future Nuclear Systems,” Proc. GLOBAL 2003, American Nuclear Society (2003) 1066-1067.

134. J.V. Shennan, “Dispersed Ceramic Fuels for the Advanced Gas-Cooled Reactor,” p. 96110 in Preparation of Nuclear Fuels, Nuclear Engineering Part XVIII, eds. A.D. Tevebaugh and D.L. Keller, Chemical Engineering Progress Symposium Series No. 80, Vol. 63 (1967).

135. J. Gan, M.K. Meyer, R.C. Birtcher, T.R. Allen, "Microstructure Evolution in ZrC Irradiated with Kr Ions,” J. ASTM Int. [3] 4 (2005).

136. Advanced Accelerator Applications program, "Candidate Approaches for an Integrated Nuclear Waste Management Strategy - Scoping Evaluations,” AAA-PDOGEN- 01-0051, Revised November 2001.

137. Roger H. Bezdek and Robert M. Wendling, "A Half Century of Long-Range Energy Forecasts: Errors Made, Lessons Learned, and Implications for Forecasting,” Journal of Fusion Energy, 21, no. 3 and 4, December 2002.

138. Emory D. Collins and John-Paul Renier, "Evaluation of Actinide Partitioning and Transmutation in Light-Water Reactors,” Atalanta Conference, Nimes France, June 2004.

139. William S. Charlton, Ryan F. LeBouf, and Sukesh Aghara, "Proliferation Resistance Assessment Method for Analyzing ATW Fuel Cycles,” ANS meeting, San Diego, 2003.

140. M. Delpech, F. Carre, Ph. Brossard, J. P. Grouiller, O. Boucher, P. Bourdot, "Preliminary Analysis of Prospective Scenarios of Transition from GenII-III Towards Gen IV Systems in France During the 21st Century,” DEN/DDIN/RTDPSF/ 2004/DO 609.

141. R. D. Lawrence, "The DIF3D Nodal Neutronics Option for Two- and ThreeDimensional Diffusion Theory Calculations in Hexagonal Geometry,” ANL-83-1, Argonne National Laboratory (1983). 
Utilization of MAs as a Fuel Component for Ultra-Long Life VHTR Configurations: Designs, Advantages and Limitations

Project 05-094

Final Scientific/Technical Report

142. B. W. Dixon, S. J. Piet, "Impact of Nuclear Energy Futures on Advanced Fuel Cycle Options,” Americas Nuclear Energy Symposium, Miami, Fl, October 2004.

143. S. J. Piet, B. W. Dixon, A. Goldmann, R. N. Hill, J. J. Jacobson, G. E. Matthern, J. D. Smith, A. M. Yacout. "Current Comparison of Advanced Fuel Cycle Options". INL/CON-05-00757. March (2006).

144. Yucca Mountain Science and Engineering Report, U.S. Department of Energy, Office of Civilian Radioactive Waste Management, DOE/RW-0539, May 2001.

145. Report to Congress - Advanced Fuel Cycle Initiative: Objectives, Approach, and Technology Summary, U.S. Department of Energy, Office of Nuclear Energy, Science, and Technology, May 2005.

146. Advanced Fuel Cycle Initiative (AFCI) Comparison Report, FY 2005, U.S. Department of Energy, Office of Nuclear Energy, Science and Technology, May 2005.

147. Advanced Fuel Cycle Initiative (AFCI) Comparison Report, FY 2006 Update, U.S. Department of Energy, Office of Nuclear Energy, July 2006.

148. S. Fetter, E. T. Chang, F. M. Mann, "Long-Term Radioactive Waste from Fusion Reactors: Part II,” Fusion Engineering and Design, 13 (1990, pp. 239-246. “Generation IV Roadmap - Technology Goals for Generation IV Nuclear Energy Systems,” GIF-01900, December, 2002.

149. G. Gigerenzer, Peter Todd, and the ABC Research Group, Simple Heuristics that Make Us Smart, Oxford University Press, 1999.

150. Andrew S. Goldmann, "Inert Matrix Fuel Burnup Calculations using a Multi-Recycle Strategy in Light Water Reactors," INL/EXT-05-Xxxxx (draft), August 2005. H. A. Grunder, B. D. Shipp, M. R. Anastasio, P. Nanos, W. J. Madia, C. P. Robinson, ”Nuclear Energy: Power for the 21st Century - An Action Plan,” April 30, 2003.

151. “Hanford Site Solid Waste Acceptance Criteria,” HNF-EP-0063 Rev 11.

152. J. Stephen Herring, "Uranium and Thorium Resources,” in The Encyclopedia of Energy, Cutler J. Cleveland, editor in chief, Academic Press, 2004.

153. W. G. Halsey, Advanced Fuel Cycle Initiative (AFCI) Repository Impact Evaluation FY05 Progress Report, UCRL-TR-215380, September 15, 2005

154. R. N. Hill, et al., "Multiple Tier Fuel Cycle Studies for Waste Transmutation,” Proc. ICONE 10, April 14-18, 2002, Arlington, VA, USA.

155. R. N. Hill, “Comparison of Fast Reactor Potential,” ANL-AFCI-115, September 30, 2003. 
Utilization of MAs as a Fuel Component for Ultra-Long Life VHTR Configurations: Designs, Advantages and Limitations

Project 05-094

Final Scientific/Technical Report

156. R. N. Hill, E. A. Hoffman, W. S. Yang, "Systematic Intercomparison of Transmutaiton Concepts,” ANL-AFCI-133, September 2004.

157. E. A. Hoffman, "Preliminary Report on Blending Strategies for Inert-Matrix Recycling in LWRs”, Argonne National Laboratory, ANL-AFCI-149, August, 2004.

158. T. K. Kim, "PWR Spent Nuclear Fuel Composition and Decay Heat Values for System Scenario Studies,” July 18, 2003.

159. H. Henryson II, B. J. Toppel, and C. G. Stenberg, "MC2-2: A Code to Calculated Fast Neutron Spectra and Multigroup Cross Sections,” ANL-8144, Argonne National Laboratory (1976).

160. “The Future of Nuclear Power - An Interdisciplinary Study,” MIT, 2004.

161. Annex: Attributes of Proliferation Resistance for Civilian Nuclear Power Systems, Nuclear Energy Research Advisory Committee (NERAC), October 2000.

162. Technological Opportunities to Increase the Proliferation Resistance of Global Civilian Nuclear Power Systems (TOPS), Report by the TOPS Task Force of the Nuclear Energy Research Advisory Committee (NERAC), January 2001.

163. "Final Report of the Panel to Review the Spent Fuel Standard for Disposition of Excess Weapons Plutonium, Committee on International Security and Arms Control,” National Academy of Sciences, Washington, DC, 2000.

164. The May 2001 National Energy Policy states on pages 5-17 and 5-22 that "the United States will continue to discourage the accumulation of separated plutonium, worldwide.”

165. Draft Environmental Impact Statement on 10 CFR Part 61, "Licensing Requirements for Land Disposal of Radioactive Waste,” NUREG-782, Volume 2. September 1981.

166. Licensing Requirement for Land Disposal of Radioactive Waste, 1982.

167. L. Soffer, S. B. Burson, C. M. Ferrell, R. Y. Lee, J. N. Ridgeley, “Accident Source Terms for Light-Water Nuclear Power Plants,” NUREG-1465, February 1995.

168. Nuclear Waste Policy Act of 1982. The or ned Jan. 7, 1983 (Public Law 97-425; 96 Stat. 2201), then amended by P.L. 100-203, Title V, Subtitle A (December 22, 1987), P.L. 100-507 (October 18, 1988), and P.L. 102-486 (The Energy Policy Act of 1992, October 24, 19 nerally codified at 42 U.S.C. 10101 and following.

169. M. T. Peters, R. N. Hill, and A. M. Yacout, "Advanced Fuel Cycle Scenarios for Nuclear Waste Management," to be presented at the ANS Annual Meeting in Washington DC, November 13-17, 2005. 
Utilization of MAs as a Fuel Component for Ultra-Long Life VHTR Configurations: Designs, Advantages and Limitations

Project 05-094

Final Scientific/Technical Report

170. $\quad$ S. J. Piet, P. L. Gibson, J. C. Joe, T. A. Kerr, Robert L. Nitschke, M. E. Dakins, "Making Sustainable Decisions Using the KONVERGENCE Framework," Waste Management 2003, February 23-27, 2003,

171. S. J. Piet, B. W. Dixon, R. G. Bennett, J. D. Smith, R. N. Hill, ”Answering Key Fuel Cycle Questions,” Americas Nuclear Energy Symposium, Miami, Fl, October 2004.

172. B. J. Toppel, “ A User’s Guide to the REBUS-3 Fuel Cycle Analysis Capability,” ANL83-2, Argonne National Laboratory (Salvatores1998 M. Salvatores, et al, The Transmutation of Long-Lived Fission Products by Neutron Irradiation, Nuclear Science and Engineering, 130, pages 309-319, 1998.

173. M. Salvatores, et al, "Systematic Assessment of LWR Recycle Strategies," ANLAFCI100, September 2003.

174. M. Salvatores, et al., "The Physics of TRU Transmutation - A Systematic Approach to the Intercomparison of Systems,” PHYSOR2004, April 2004.

175. M. A. Smith, E. E. Morris and R. N. Hill, "Physics and Safety Studies of Low Conversion Ratio Sodium Cooled Fast Reactors,” Nuclear Technology: Achieving Global Economic Growth While Safeguarding the Environment, New Orleans, LA, Nov. 16-20, 2003, American Nuclear Society.

176. Julian J. Steyn, “Uranium Resources: Need For 21st Century Advanced Fuel Cycles,” Energy Resources International, Inc., NEI International Fuel Seminar 2003.

177. J. A. Stillman, Homogeneous Recycling Strategies in LWRs for Plutonium, Neptunium, and Americium Management, Argonne National Laboratory, ANLAFCI- 124, August, 2004.

178. J. A. Stillman, Private Communications, Argonne National Laboratory, 2004.

179. J. Stillman, T. H. Bauer, R. N. Hill, R. A. Wigeland, "Follow-Up Analyses for the ANTT Review,” ANL-AFCI-132, September 2004.

180. J. Stillman, "Intrinsic Proliferation Resistance Metrics for Separated Streams of ReactorGrade Transuranics” October 25, 2004.

181. T. A. Taiwo and R. N. Hill, Summary of Generation IV Transmutation Impacts, ANLAFCI-150, June 30, 2005.

182. Personal communications to system analysis colleagues, summer 2004.

183. G. F. Vandergrift, et al, Lab-Scale Demonstration of the UREX+ Process, Waste Management 2004, Tucson, AZ, February, 2004. 
Utilization of MAs as a Fuel Component for Ultra-Long Life VHTR Configurations: Designs, Advantages and Limitations

Project 05-094

Final Scientific/Technical Report

184. Matthew L. Wald, A New Vision for Nuclear Waste, Technology Review, December 2004, pp. 38-44.

185. F. William Walker, J. R. Parrington, and F. Feiner, Nuclides and Isotopes, 14th Edition, 1989.

186. Alan E. Waltar, Radiation and Modern Life: Fulfilling Marie Curie's Dream, Prometheus Books, New York, 2004

187. Alan E. Waltar and Ronald P. Omberg, editors, “An Evaluation of the Proliferation Resistant Characteristics of Light Water Reactor Fuel with the Potential for Recycle in the United States,” Blue Ribbon Committee, November 2004.

188. R. A. Wigeland, T. H. Bauer, T. H. Fanning, and E. E. Morris, "Repository Impact of LWR MOX and Fast Reactor Recycling Options,” Proceedings of Global 2003, ANS/ENS International Winter Meeting, New Orleans, LA, November 16-20, 2003.

189. R.A. Wigeland, T.H. Bauer, T.H. Fanning, and E.E. Morris, “Spent Nuclear Fuel Separations and Transmutation Criteria for Benefit to a Geologic Repository,” Waste Management ’04, Tucson, AZ, February 2004.

190. R.A. Wigeland and T.H. Bauer, "Repository Benefits of AFCI Options," ANL-AFCI129, September 3, 2004.

191. R. A. Wigeland, T. Bauer, E. Morris, "Repository Benefits,“ presentation to AFCI system analysis working group, Salt Lake City, June 2, 2005.

192. R. A. Wigeland, et al, "Separations and Transmutation Criteria to improve Utilization of a Geologic Repository,” Nuclear Technology, Vol. 154, April 2006.

193. WIMS - A Modular Scheme for Neutronics Calculations, User's Guide for Version 8, Serco Assurance.

194. System Dynamics Analysis of the AFCI Scenarios, Progress Report, ANL-AFCI- 131, September 2004.

195. W. S. Yang, Y. Kim, R. N. Hill, T. A. Taiwo, and H. S. Khalil, "Long-Lived Fission Product Transmutation Studies,” Nuclear Science and Engineering, 146, pages 291- 318, 2004.

196. D. C. WADE, R. N. HILL, “The Design Rationale of the IFR,” Progress in Nuclear Energy, 31, 1-2, 13-42 (1997).

197. R. A. WIGELAND and T. H. BAUER, "Repository Benefits of AFCI Options," Argonne National Laboratory, ANL-AFCI-129, September (2004). 
198. A. E. DUBBERLEY, K. YOSHIDA, C. E. BOARMAN, and T. WU, "SuperPRISM Oxide and Metal Fuel Core Designs,” Proc. of ICONE 8, 8th International Conference on Nuclear Engineering (2000).

199. R. N. HILL, D. C. WADE, E. K. FUJITA, and H. KHALIL, "Physics Studies of Higher Actinide Consumption in an LMR," Proc. Int. Conf. on the Physics of Reactors, Marseille, France, p.I-83, April 23-27 (1990).

200. R. N. HILL, D. C. WADE, J. R. LIAW, and E. K. FUJITA, "Physics Studies of Weapons Plutonium Disposition in the Integral Fast Reactor Closed Fuel Cycle,” Nuclear Science and Engineering, 121, 17 (1995).

201. E. A. HOFFMAN, W. S. YANG, and R. N. HILL, "Preliminary Core Design Studies for the Advanced Burner Reactor over a Wide Range of Conversion Ratios," Argonne National Laboratory, ANL-AFCI-177 (2006).

202. M. A. SMITH, E. E. MORRIS, R. N. HILL, "Physics and Safety Studies of Low Conversion Ratio Sodium Cooled Fast Reactors”, Global 2003, New Orleans, Louisiana, United States, pp. 423-433, November 16-20 (2003).

203. H. HENRYSON II, B. J. TOPPEL, and C. G. STENBERG, "MC2-2: A Code to calculate Fast Neutron Spectra and Multi-Group Cross-Sections.” ANL-8144, Argonne National Laboratory (1976).

204. B. J. Toppel, “A User's Guide to the REBUS-3 Fuel Cycle Analysis Capability,” ANL83-2, Argonne National Laboratory (1983).

205. B. E. LAHM, J. F. KOENIG, R. G. PAHL, D. L. PORTER, and D. C. CRAWFORD, "Experience with Advanced Driver Fuels in EBR-II,” Journal of Nuclear Materials, 204, 119 (1993).

206. R. B. BAKER, F. E. BARD, R. D. LEGGETT, and A. L. PINTER, "Status of Fuel, Blanket, and Absorber Testing in the Fast Flux Test Facility," Journal of Nuclear Materials, 204, 109 (1993).

207. A. L. PINTER and R. B. BAKER, "Metal Fuel Test Program in the FFTF," Journal of Nuclear Materials, 204, 124 (1993).

208. T. A. Taiwo, G. Palmiotti, G. Aliberti, M. Salvatores, T. Kim, "Uncertainty and target accuracy studies for the very high temperature reactor(VHTR) physics parameters." ANL-GENIV-051. September (2006).

209. Burchell, T., Bratton, R., Windes, W. "NGNP Graphite Selection and Acquisition Strategy”. April 2008. 
210. Bunn, M., et. al. “Economics of Reprocessing vs. Direct Disposal of Spent Nuclear Fuel”. Harvard University, 2003

211. D. Petti, et al, “Technical Program Plan for the Advanced Gas Reactor Fuel Development and Qualification Program”, INEEL/CON-04-02416, , May 2005.

212. Bays, S., "Physics Characterization of a Heterogeneous Sodium Fast Reactor Transmutation System”. DE-AC07-99ID-13727. September 2007.

213. A. Hoffman, "Preliminary Report on Blending Strategies for Inert-Matrix Recycling in LWRs”, Argonne National Laboratory, ANL-AFCI-149, August, 2005.

214. Morris, E., Bauer, T., “Modeling of the Repository Behavior of TRISO Fuel.” ANLAFCI-160. (2005).

215. Wigeland, R. A. ; Bauer, T. H.. “Repository benefits of AFCI options”. ANL-AFCI-129. (2005).

216. Taiwo, T., Fatone, M., Palmiotti G., Hill, R., "Physics Studies for a Particle Bed Gas Cooled Fast Reactor Core Design. (2002).

217. Emory D. Collins and John-Paul Renier, "Systems Studies of Actinide PartitioningTransmutation Recycle Methods”, Oak Ridge National Laboratory, Proceedings of GLOBAL 2005, Tsukuba, Japan, Oct 9-13, 2005.

218. Del Cul, G.D., "TRISO-Coated Fuel Processing to Support High Temperature GasCooled Reactors,” ORNL/TM-2002/156. (2002). 


\section{APPENDIX A. Project Publications}

\subsection{M.S Theses}

1. D. E. Ames II, "Configuration Adjustment Potential of the VHTR Prismatic Cores with Advanced Actinide Fuels”, M.S. Thesis, August 2006, Texas A\&M University (2006). Advisor - P. V. Tsvetkov

2. T. G. Lewis III, "Analysis of TRU-Fueled VHTR Prismatic Core Performance Domains”, M.S. Thesis, December 2007, Texas A\&M University (2007). Advisor - P. V. Tsvetkov

3. M. L. Pritchard, "Neutronics Analysis of Pebble Bed Cores with Transuranics", M.S. Thesis, December 2007, Texas A\&M University (2007). Advisor - P. V. Tsvetkov

4. A. B. Alajo, "Impact of PWR Spent Fuel Variations on TRU-Fueled VHTRs", M.S. Thesis, December 2007, Texas A\&M University (2007). Advisor - P. V. Tsvetkov

\subsection{Refereed Journal Papers}

5. P. V. Tsvetkov, D. E. Ames II, A. B. Alajo, T. G. Lewis III, "Spectrum Shifting as a Mechanism to Improve Performance of VHTRs with Advanced Actinide Fuels", Nucl. Eng. Des., 238 (2008), pp. 1958-1964 (2008).

6. P. V. Tsvetkov, A. B. Alajo, D. E. Ames II, “Autonomous Control Strategies for VHTR-Based Systems for Hydrogen Production”, J. Eng. for Gas Turbines and Power, July 2009, 131 (2009).

7. P. V. Tsvetkov, T. G. Lewis III, A. B. Alajo, "TRU-Fueled VHTRs for Applications Requiring an Extended Operation with Minimized Control and No Refueling”, J. Eng. for Gas Turbines and Power, July 2009, 131 (2009).

8. T. G. Lewis III, P. V. Tsvetkov, "Physics Features of TRU-Fueled VHTRs", Science and Technology of Nuclear Installations (2009). 
Utilization of MAs as a Fuel Component for Ultra-Long Life VHTR Configurations: Designs, Advantages and Limitations

Project 05-094

Final Scientific/Technical Report

9.3. Refereed Full Conference Papers

9. P. V. Tsvetkov, "Coupled Hybrid Monte Carlo - Deterministic Analysis of VHTR Configurations with Advanced Actinide Fuels", Proc. Intern. Congr. Adv. Nucl. Power Plants (ICAPP 06), Reno, Nevada, June 4 - 8, 2006, ANS Embedded Topical Meeting, 2006 ANS Annual Meeting, pp. 1984 - 1990 (2006).

10. M. L. Pritchard, P. V. Tsvetkov, "Pebble-Bed Core Design Option for VHTRs Core Configuration Flexibility and Potential Applications”, Proc. ANS Topical Meeting/Intern. Conf. on Reactor Physics (PHYSOR 2006), Vancouver, Canada, September 10 - 14, 2006, paper D072, pp. 1 - 5 (2006).

11. P. V. Tsvetkov, D. E. Ames II, A. B. Alajo, M. L. Pritchard, "Self-Sustainability of VHTR Configurations with Advanced Actinide Fuels”, Proc. ANS Topical Meeting/Intern. Conf. on Reactor Physics (PHYSOR 2006), Vancouver, Canada, September 10 - 14, 2006, paper D071, pp. 1 - 10 (2006).

12. P. V. Tsvetkov, D. E. Ames II, M. L. Pritchard, A. B. Alajo, T. G. Lewis III, "TRU-fueled VHTRs: Design, Performance and Applications", Proc. ANS Topical Meeting/Intern. Conf. on Advanced Nuclear Fuel Cycles and Systems (GLOBAL 2007), September 9-13, 2007, Boise, Idaho, USA, pp 852 - 855, ANS, Omnipress (2007).

13. P. V. Tsvetkov, A. B. Alajo, T. G. Lewis III, D. E. Ames II, "Out-of-Core Fuel Cycle Characteristics of VHTRs with No On-Site Refueling”, Proc. Intern. Congr. Adv. Nucl. Power Plants (ICAPP 08), Anaheim, California, June 8 - 12, 2008, ANS Embedded Topical Meeting, 2008 ANS Annual Meeting, paper 8233, pp. 298 - 302, ANS (2008).

14. A. B. Alajo, P. V. Tsvetkov, "Core Lifetime and Fuel Utilization in Prismatic VHTR Cores”, Proc. ANS Topical Meeting/Intern. Conf. on Reactor Physics (PHYSOR 2008), Interlaken, Switzerland, September 14 - 19, 2008, paper 569, pp 1 - 6, Paul Scherrer Institute, ISBN 978-3-9521409-5-6, PSI (2008).

15. P. V. Tsvetkov, "High-Fidelity System Modeling of Advanced Nuclear Energy Systems Approaching a Zero-Nuclear-Waste Limit”, Advances in Nuclear Fuel Management IV (ANFM 2009), Hilton Head Island, South Carolina, USA, April 12-15, 2009, on CD-ROM, American Nuclear Society, LaGrange Park, IL (2009).

16. P. V. Tsvetkov, D. E. Ames, M. L. Pritchard, A. B. Alajo, "Spectrum Shifting as a Mechanism to Improve Performance of VHTRs with Advanced Actinide Fuels”, 
Utilization of MAs as a Fuel Component for Ultra-Long Life VHTR Configurations: Designs, Advantages and Limitations

Proc. $14^{\text {th }}$ Intern. Conf. on Nucl. Eng. (ICONE 14), July 17 - 20, 2006, Miami, Florida, USA, paper ICONE14-89563, pp. 1 - 6, ASME (2006).

17. P. V. Tsvetkov, T. G. Lewis III, A. B. Alajo, "TRU-Fueled VHTRs for Applications Requiring an Extended Operation with Minimized Control and No Refueling”, Proc. $16^{\text {th }}$ Intern. Conf. on Nucl. Eng. (ICONE 16), Orlando, Florida, May 11-15, 2008, paper ICONE16-48334, pp. 1 - 11 ASME (2008).

18. D. E. Ames II, P. V. Tsvetkov, "Benchmark Efforts to Support Studies of Advanced VHTRs”, Proc. $4^{\text {th }}$ Intern. Conf. on HTR Technology (HTR2008), Sept. 28 - Oct. 1, 2008, Washington D.C., USA, paper HTR2008-58206, pp. 1 - 8, ASME (2008).

19. P. V. Tsvetkov, D. E. Ames II, A. B. Alajo, T. G. Lewis III, "VHTR-Based Systems for Autonomous Co-Generation Applications", Proc. $4^{\text {th }}$ Intern. Conf. on HTR Technology (HTR2008), Sept. 28 - Oct. 1, 2008, Washington D.C., USA, paper HTR2008-58228, pp. 1 - 9, ASME (2008).

20. P. V. Tsvetkov, A. B. Alajo, D. E. Ames II, “Autonomous Control Strategies for VHTR-based Systems for Hydrogen Production”, Proc. $4^{\text {th }}$ Intern. Conf. on HTR Technology (HTR2008), Sept. 28 - Oct. 1, 2008, Washington D.C., USA, paper HTR2008-58231, pp. 1 - 7, ASME (2008).

21. P. V. Tsvetkov, T. G. Lewis III, A. B. Alajo, D. E. Ames II, “”Used Fuel” Vectors and Waste Minimization Strategies for VHTRs Operating without Refueling”, Proc. $4^{\text {th }}$ Intern. Conf. on HTR Technology (HTR2008), Sept. 28 - Oct. 1, 2008, Washington D.C., USA, paper HTR2008-58233, pp. 1 - 10, ASME (2008).

\subsection{Refereed Conference Summaries (ANS Transactions)}

22. P. V. Tsvetkov, M. L. Pritchard, D. E. Ames II, A. B. Alajo, "Analysis of the Pebble-Bed VHTR Spectrum Shifting Capabilities for Advanced Fuel Cycles", Trans. Amer. Nucl. Soc., 94, pp. 403 - 404, USA (2006).

23. P. V. Tsvetkov, D. E. Ames II, A. B. Alajo, “Configuration Adjustment Potential of the VHTR Prismatic Cores with Advanced Actinide Fuels”, Trans. Amer. Nucl. Soc., 94, pp. 597 - 598, USA (2006).

24. P. V. Tsvetkov, A. B. Alajo, "Potential of Minor Actinides to Enhance VHTR Performance Characteristics”, Trans. Amer. Nucl. Soc., 94, pp. 93 - 94, USA (2006). 
Utilization of MAs as a Fuel Component for Ultra-Long Life VHTR Configurations: Designs, Advantages and Limitations

25. M. L. Pritchard, P. V. Tsvetkov, A. B. Alajo, "Neutronics Analysis of Pebble-Bed Cores with TRUs”, Trans. Amer. Nucl. Soc., 96, pp. 827 - 828, USA (2007).

26. P. V. Tsvetkov, D. E. Ames II, M. L. Pritchard, "Utilization of TRUs as a Fuel for VHTRs: Compositions, Neutronics Impact and Safety”, Trans. Amer. Nucl. Soc., 96, pp. 769 - 770, USA (2007).

27. T. G. Lewis III, D. E. Ames II, P. V. Tsvetkov, “Analysis of TRU-Fueled VHTR Prismatic Core Performance Domains”, Trans. Amer. Nucl. Soc., 97, pp. 859 860, USA (2007).

28. A. B. Alajo, P. V. Tsvetkov, "Reactor Physics of VHTRs with $\mathrm{UO}_{2}$, UCO \& $\mathrm{UC}_{0.5} \mathrm{O}_{1.5}$ Kernels Operating without Onsite Refueling”, Trans. Amer. Nucl. Soc., 98, pp. 736 - 737, USA (2008).

29. P. V. Tsvetkov, T. G. Lewis III, A. B. Alajo, "Utilization of TRUs in VHTRs Operation in a Single-Batch Mode: Front End, Back End, and Performance”, Trans. Amer. Nucl. Soc., 98, pp. 733 - 734, USA (2008).

30. P. V. Tsvetkov, "LWR-VHTR Fuel Cycles with Dedicated Small $14 \mathrm{MeV}$ Waste Incineration Back-End Clean-Up Facilities”, Trans. Amer. Nucl. Soc., 99, pp. 220 221 (2008). 


\section{APPENDIX B. Prototype Specifications of Small-Scale VHTRs}

\subsection{HTR-10 Configuration (Pebble-Bed Core)}

\section{Table 13. Specification of the HTR-10 Design}

\begin{tabular}{|c|c|}
\hline Reactor thermal power & $10 \mathrm{MW}$ \\
\hline Primary helium pressure & $3.0 \mathrm{MPa}$ \\
\hline Active core volume & $5 \mathrm{~m}^{3}$ \\
\hline Reactor core diameter & $180 \mathrm{~cm}$ \\
\hline Average core height & $197 \mathrm{~cm}$ \\
\hline Average helium temperature at reactor outlet & $700^{\circ} \mathrm{C}$ \\
\hline Average helium temperature at reactor inlet & $250^{\circ} \mathrm{C}$ \\
\hline Helium mass flow rate at full power & $4.3 \mathrm{~kg} / \mathrm{s}$ \\
\hline Main steam pressure at steam generator outlet & $4.0 \mathrm{MPa}$ \\
\hline Main steam temperature at steam generator & $440^{\circ} \mathrm{C}$ \\
\hline Feed water temperature & $104^{\circ} \mathrm{C}$ \\
\hline Fuel-to-graphite ball ratio & $0.57 / 0.43$ \\
\hline Number of control rods in side reflector & 10 \\
\hline Number of absorber ball units in side reflector & 7 \\
\hline Nuclear fuel & $\mathrm{UO}_{2}$ \\
\hline Heavy metal loading per fuel element & $5 \mathrm{~g}$ \\
\hline Enrichment of fresh fuel element & $17 \%$ \\
\hline Number of fuel elements in equilibrium core & 27,000 \\
\hline Fuel loading mode & multi-pass \\
\hline \multicolumn{2}{|c|}{ Fuel Element (Fuel Pebble) Characteristics, 8,300 Particles per Fuel Pebble } \\
\hline Diameter of ball & $6.0 \mathrm{~cm}$ \\
\hline Diameter of fuel zone & $5.0 \mathrm{~cm}$ \\
\hline Density of graphite in matrix and outer shell & $1.73 \mathrm{~g} / \mathrm{cm}^{3}$ \\
\hline Heavy metal (uranium) loading (weight) per ball & $5.0 \mathrm{~g}$ \\
\hline Enrichment of U-235 (weight) & $17 \%$ \\
\hline
\end{tabular}




\section{Utilization of MAs as a Fuel Component for Ultra-Long Life VHTR Configurations: Designs, Advantages and Limitations}

Project 05-094

Final Scientific/Technical Report

\begin{tabular}{|c|c|}
\hline Equivalent natural boron content of impurities in uranium & $4 \mathrm{ppm}$ \\
\hline Equivalent natural boron content of impurities in graphite & $1.3 \mathrm{ppm}$ \\
\hline Volumetric filling fraction of balls in the core & 0.61 \\
\hline \multicolumn{2}{|l|}{ Fuel kernel } \\
\hline Radius of the kernel & $0.02500 \mathrm{~cm}$ \\
\hline $\mathrm{UO}_{2}$ density & $10.4 \mathrm{~g} / \mathrm{cm}^{3}$ \\
\hline \multicolumn{2}{|l|}{ TRISO Coatings } \\
\hline Coating layer materials (starting from kernel) & $\mathrm{PyC} / \mathrm{PyC} / \mathrm{SiC} / \mathrm{PyC}$ \\
\hline Coating layer thickness (mm) & $0.090 / 0.040 / 0.035 / 0.040$ \\
\hline Coating layer density $\left(\mathrm{g} / \mathrm{cm}^{3}\right)$ & $1.10 / 1.90 / 3.18 / 1.90$ \\
\hline \multicolumn{2}{|l|}{ Dummy (no fuel) elements } \\
\hline Diameter of ball & $6.0 \mathrm{~cm}$ \\
\hline Density of graphite & $1.73 \mathrm{~g} / \mathrm{cm}^{3}$ \\
\hline Equivalent natural boron content of impurities in graphite & $1.3 \mathrm{ppm}$ \\
\hline \multicolumn{2}{|c|}{ Additional Reactor Core Parameters } \\
\hline Density of reflector graphite & $1.76 \mathrm{~g} / \mathrm{cm}^{3}$ \\
\hline Equivalent natural boron impurity in reflector graphite & $4.8366 \mathrm{ppm}$ \\
\hline Density of boronated carbon brick including $\mathrm{B}_{4} \mathrm{C}$ & $1.59 \mathrm{~g} / \mathrm{cm}^{3}$ \\
\hline Weight ratio of $\mathrm{B}_{4} \mathrm{C}$ in boronated carbon brick & $5 \%$ \\
\hline
\end{tabular}


Utilization of MAs as a Fuel Component for Ultra-Long Life

VHTR Configurations: Designs, Advantages and Limitations

Project 05-094

Final Scientific/Technical Report

\subsection{HTTR Configuration (Prismatic Block Core)}

Table 14. Specification of the HTTR Design

\begin{tabular}{|c|c|}
\hline Thermal Power & $30 \mathrm{MW}$ \\
\hline Outlet coolant temperature / Inlet coolant temperature $\left({ }^{\circ} \mathrm{C}\right)$ & $950 / 395$ \\
\hline Primary coolant pressure & $4 \mathrm{MPa}$ \\
\hline Core structure material & Graphite \\
\hline Equivalent core diameter / Effective core height (cm) & $230 / 290$ \\
\hline Average power density & $2.5 \mathrm{~W} / \mathrm{cc}$ \\
\hline \multicolumn{2}{|l|}{ Fuel } \\
\hline Material & $\mathrm{UO}_{2}$ \\
\hline Uranium enrichment & 3 to $10 \mathrm{wt} . \%$ \\
\hline Type of fuel & Pin-in-block \\
\hline Burn-up period & 660 days \\
\hline Coolant material & Helium gas \\
\hline Flow direction in core & Downward \\
\hline \multicolumn{2}{|l|}{ Reflector thickness } \\
\hline Top / Side / Bottom (cm) & $116 / 99$ / 116 \\
\hline Number of fuel assemblies & 150 \\
\hline Number of fuel columns & 30 \\
\hline \multicolumn{2}{|l|}{ Number of pairs of control rods } \\
\hline Total & 16 \\
\hline In core & 7 \\
\hline In reflector & 9 \\
\hline \multicolumn{2}{|c|}{ Fuel Element (Compact) Characteristics, 13,000 Particles per Fuel Compact } \\
\hline Type & Hollow cylinder \\
\hline Material & Graphite \\
\hline Number of particles per fuel compact & 13000 \\
\hline Number of fuel compacts per fuel rod & 14 \\
\hline Graphite matrix density & $1.690 \mathrm{~g} / \mathrm{cc}$ \\
\hline Graphite matrix Impurity & $0.82 \mathrm{ppm} \mathrm{B}$ \\
\hline
\end{tabular}




\section{Utilization of MAs as a Fuel Component for Ultra-Long Life} VHTR Configurations: Designs, Advantages and Limitations

Project 05-094

Final Scientific/Technical Report

\begin{tabular}{|c|c|c|}
\hline Diameter-inner / Diameter-outer (cm) & \multicolumn{2}{|c|}{$1.0 / 2.6$} \\
\hline Height & \multicolumn{2}{|l|}{$3.9 \mathrm{~cm}$} \\
\hline \multicolumn{3}{|l|}{ Fuel kernel } \\
\hline Radius of the kernel & \multicolumn{2}{|c|}{$0.02985 \mathrm{~cm}$} \\
\hline $\mathrm{UO}_{2}$ density & \multicolumn{2}{|c|}{$10.41 \mathrm{~g} / \mathrm{cm}^{3}$} \\
\hline \multicolumn{3}{|l|}{ TRISO Coatings } \\
\hline Coating layer materials (starting from kernel) & \multicolumn{2}{|c|}{ PyC / PyC / SiC / PyC } \\
\hline Coating layer thickness (mm) & \multicolumn{2}{|c|}{$0.060 / 0.031 / 0.029 / 0.046$} \\
\hline Coating layer density $\left(\mathrm{g} / \mathrm{cm}^{3}\right)$ & \multicolumn{2}{|c|}{1.14 / 1.89 / $3.20 / 1.87$} \\
\hline \multicolumn{3}{|l|}{ Dummy (no fuel) elements } \\
\hline Diameter of ball & \multicolumn{2}{|l|}{$6.0 \mathrm{~cm}$} \\
\hline Density of graphite & \multicolumn{2}{|c|}{$1.73 \mathrm{~g} / \mathrm{cm}^{3}$} \\
\hline Equivalent natural boron content of impurities in graphite & \multicolumn{2}{|c|}{$1.3 \mathrm{ppm}$} \\
\hline \multicolumn{3}{|c|}{ Fuel Rod Characteristics } \\
\hline Number of fuel particles & \multicolumn{2}{|c|}{182,200} \\
\hline Graphite matrix density & \multicolumn{2}{|c|}{$1.690 \mathrm{~g} / \mathrm{CC}$} \\
\hline Graphite matrix Impurity & \multicolumn{2}{|c|}{0.82 ppm Natural Boron } \\
\hline Diameter-inner & \multicolumn{2}{|l|}{$1.0 \mathrm{~cm}$} \\
\hline Diameter-outer & \multicolumn{2}{|l|}{$2.6 \mathrm{~cm}$} \\
\hline Effective height of fuel rod & \multicolumn{2}{|c|}{$54.6 \mathrm{~cm}$} \\
\hline \multicolumn{3}{|l|}{ Graphite Sleeve } \\
\hline Material & \multicolumn{2}{|c|}{ Graphite } \\
\hline Density & \multicolumn{2}{|c|}{$1.770 \mathrm{~g} / \mathrm{CC}$} \\
\hline Equivalent natural boron content of impurities & \multicolumn{2}{|c|}{0.37 ppm Natural Boron } \\
\hline Diameter-inner & \multicolumn{2}{|l|}{$2.6 \mathrm{~cm}$} \\
\hline Diameter-outer & \multicolumn{2}{|l|}{$3.4 \mathrm{~cm}$} \\
\hline Height & \multicolumn{2}{|c|}{$57.7 \mathrm{~cm}$} \\
\hline \multicolumn{3}{|c|}{ Burnable Poison Rod Characteristics } \\
\hline Type & H-I & H-II \\
\hline \multicolumn{3}{|l|}{ Absorber section } \\
\hline Material & $\mathrm{B}_{4} \mathrm{C}-\mathrm{C}$ & $\mathrm{B}_{4} \mathrm{C}-\mathrm{C}$ \\
\hline
\end{tabular}




\section{Utilization of MAs as a Fuel Component for Ultra-Long Life} VHTR Configurations: Designs, Advantages and Limitations

Project 05-094

Final Scientific/Technical Report

\begin{tabular}{|c|c|c|}
\hline "Density & $101.79 \mathrm{~g} / \mathrm{cc}$ & $1.82 \mathrm{~g} / \mathrm{cc}$ \\
\hline Natural boron concentration & 2.22 wt.\% & 2.74 wt.\% \\
\hline Diameter & $1.39 \mathrm{~cm}$ & $1.39 \mathrm{~cm}$ \\
\hline Height & $2.0 \mathrm{~cm}$ & $2.5 \mathrm{~cm}$ \\
\hline B-10 fraction & 18.7 wt.\% & 18.7 wt.\% \\
\hline \multicolumn{3}{|l|}{ Graphite section } \\
\hline Density & $1.77 \mathrm{~g} / \mathrm{cc}$ & $1.77 \mathrm{~g} / \mathrm{cc}$ \\
\hline Diameter & $1.40 \mathrm{~cm}$ & $1.40 \mathrm{~cm}$ \\
\hline Height & $10 \mathrm{~cm}$ & $10 \mathrm{~cm}$ \\
\hline \multicolumn{3}{|c|}{ Control Rod Characteristics } \\
\hline \multicolumn{3}{|l|}{ Neutron Absorber Sections (annular) } \\
\hline Material & \multicolumn{2}{|l|}{10} \\
\hline Density & \multicolumn{2}{|l|}{$\mathrm{B}_{4} \mathrm{C}$ and $\mathrm{C}$} \\
\hline Diameter-inner & \multicolumn{2}{|l|}{$1.9 \mathrm{~g} / \mathrm{cc}$} \\
\hline Diameter-outer & \multicolumn{2}{|l|}{$7.5 \mathrm{~cm}$} \\
\hline Height & \multicolumn{2}{|l|}{$10.5 \mathrm{~cm}$} \\
\hline Effective height & \multicolumn{2}{|l|}{$29.0 \mathrm{~cm}$} \\
\hline Spacing between neutron absorber sections & \multicolumn{2}{|c|}{$290 \mathrm{~cm}$ (10 absorber sections) } \\
\hline \multicolumn{3}{|l|}{ Control Rod Sleeve } \\
\hline Material & \multicolumn{2}{|l|}{ Alloy $800 \mathrm{H}$} \\
\hline Thickness & \multicolumn{2}{|l|}{$0.35 \mathrm{~cm}$} \\
\hline \multicolumn{3}{|l|}{ Control Rod } \\
\hline Number of control rods & \multicolumn{2}{|l|}{32 (16 pairs) } \\
\hline Number of control rods in active core & \multicolumn{2}{|l|}{14 (7 pairs) } \\
\hline Number of control rods in replaceable reflector region & \multicolumn{2}{|l|}{18 (9 pairs) } \\
\hline Diameter-inner & \multicolumn{2}{|l|}{$6.5 \mathrm{~cm}$} \\
\hline Diameter-outer & \multicolumn{2}{|l|}{$11.3 \mathrm{~cm}$} \\
\hline Height & \multicolumn{2}{|l|}{$310 \mathrm{~cm}$} \\
\hline
\end{tabular}




\section{Utilization of MAs as a Fuel Component for Ultra-Long Life} VHTR Configurations: Designs, Advantages and Limitations

Project 05-094

Final Scientific/Technical Report

\begin{tabular}{|c|c|}
\hline \multicolumn{2}{|c|}{ Fuel Graphite Block Characteristics } \\
\hline Type & Pin-in-block \\
\hline Configuration & Hexagonal \\
\hline Material & IG-110 Graphite \\
\hline Density & $1.770 \mathrm{~g} / \mathrm{cc}$ \\
\hline Impurity & 0.40 ppm Natural Boron \\
\hline Height & $58.0 \mathrm{~cm}$ \\
\hline Width across the flats & $36.0 \mathrm{~cm}$ \\
\hline Number of fuel holes in block & 33 or 31 \\
\hline Fuel hole diameter & $4.1 \mathrm{~cm}$ \\
\hline Fuel hole height & $58.0 \mathrm{~cm}$ \\
\hline Number of burnable poison holes & 3 \\
\hline Burnable poison hole diameter & $1.5 \mathrm{~cm}$ \\
\hline Burnable poison hole height & $50.0 \mathrm{~cm}$ \\
\hline \multicolumn{2}{|c|}{ Replaceable Reflector Block Characteristics } \\
\hline Configuration & Hexagonal \\
\hline Material & IG-110 Graphite \\
\hline Density & $1.760 \mathrm{~g} / \mathrm{cc}$ \\
\hline Impurity & 0.37 ppm Natural Boron \\
\hline Height & $58.0 \mathrm{~cm}$ \\
\hline Width across the flats & $36.0 \mathrm{~cm}$ \\
\hline Number of coolant holes if applicable & $33 / 31$ \\
\hline Coolant hole diameter & $4.1 \mathrm{~cm}$ \\
\hline Coolant hole height & $58.0 \mathrm{~cm}$ \\
\hline \multicolumn{2}{|c|}{ Control Rod Guide Block Characteristics } \\
\hline Material & IG-110 Graphite \\
\hline Density & $1.770 \mathrm{~g} / \mathrm{cc}$ \\
\hline Impurity & 0.40 ppm Natural Boron \\
\hline Height & $58.0 \mathrm{~cm}$ \\
\hline Width across the flats & $36.0 \mathrm{~cm}$ \\
\hline
\end{tabular}




\section{Utilization of MAs as a Fuel Component for Ultra-Long Life} VHTR Configurations: Designs, Advantages and Limitations

Project 05-094

Final Scientific/Technical Report

\begin{tabular}{|l|l|}
\hline \hline Number of control rod holes in block & 2 \\
\hline Control rod hole diameter & $12.3 \mathrm{~cm}$ \\
\hline Control rod hole height & $58.0 \mathrm{~cm}$ \\
\hline Number of reserve shutdown holes in block & 1 \\
\hline Reserve shutdown hole diameter & $12.3 \mathrm{~cm}$ \\
\hline Reserve shutdown hole height & $58.0 \mathrm{~cm}$ \\
\hline
\end{tabular}




\section{T/R \\ Research Initiative}

Utilization of MAs as a Fuel Component for Ultra-Long Life

VHTR Configurations: Designs, Advantages and Limitations

Project 05-094

Final Scientific/Technical Report

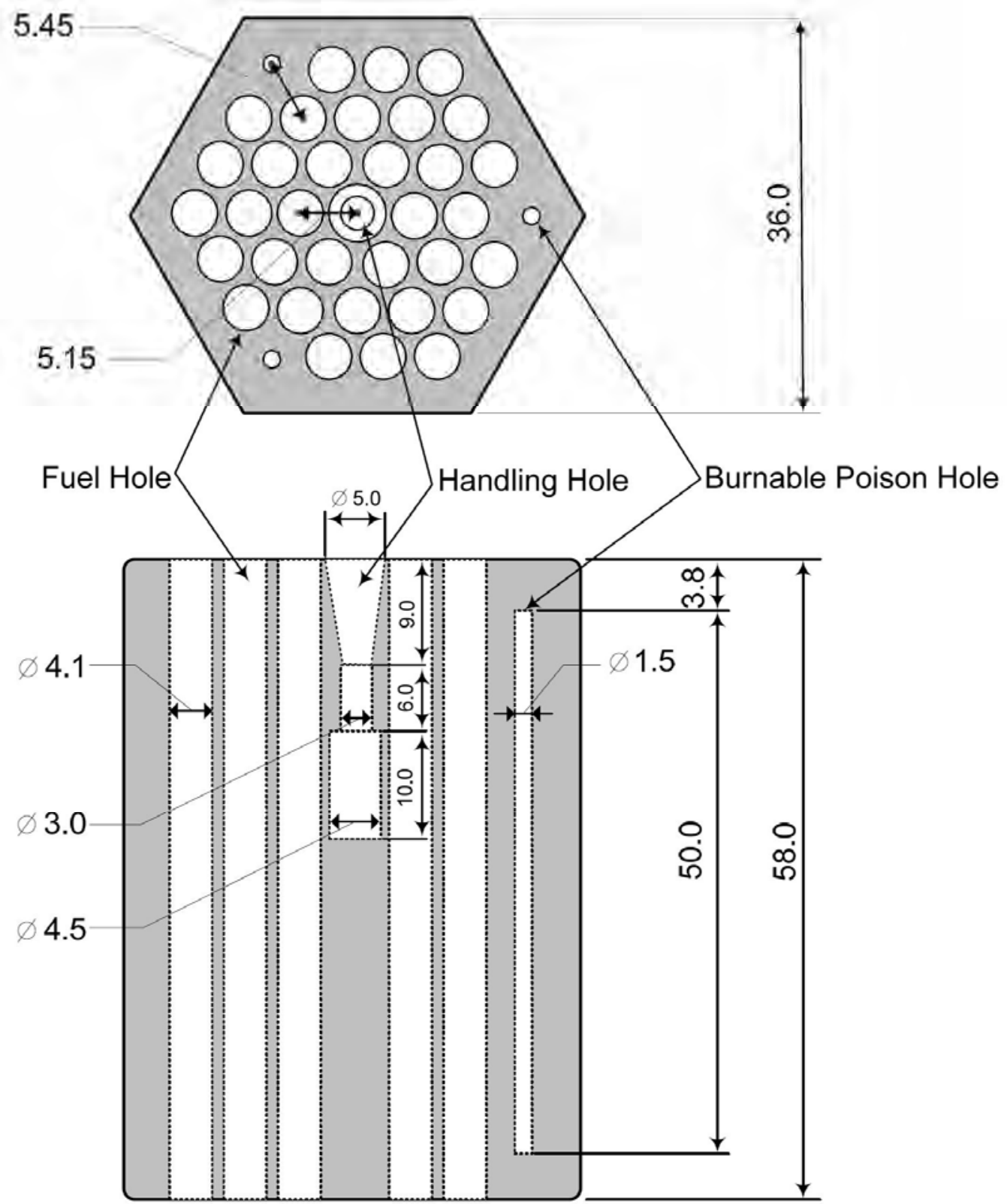

Figure 14. Fuel graphite block (measurements in cm). 
Utilization of MAs as a Fuel Component for Ultra-Long Life

VHTR Configurations: Designs, Advantages and Limitations

Project 05-094

Final Scientific/Technical Report

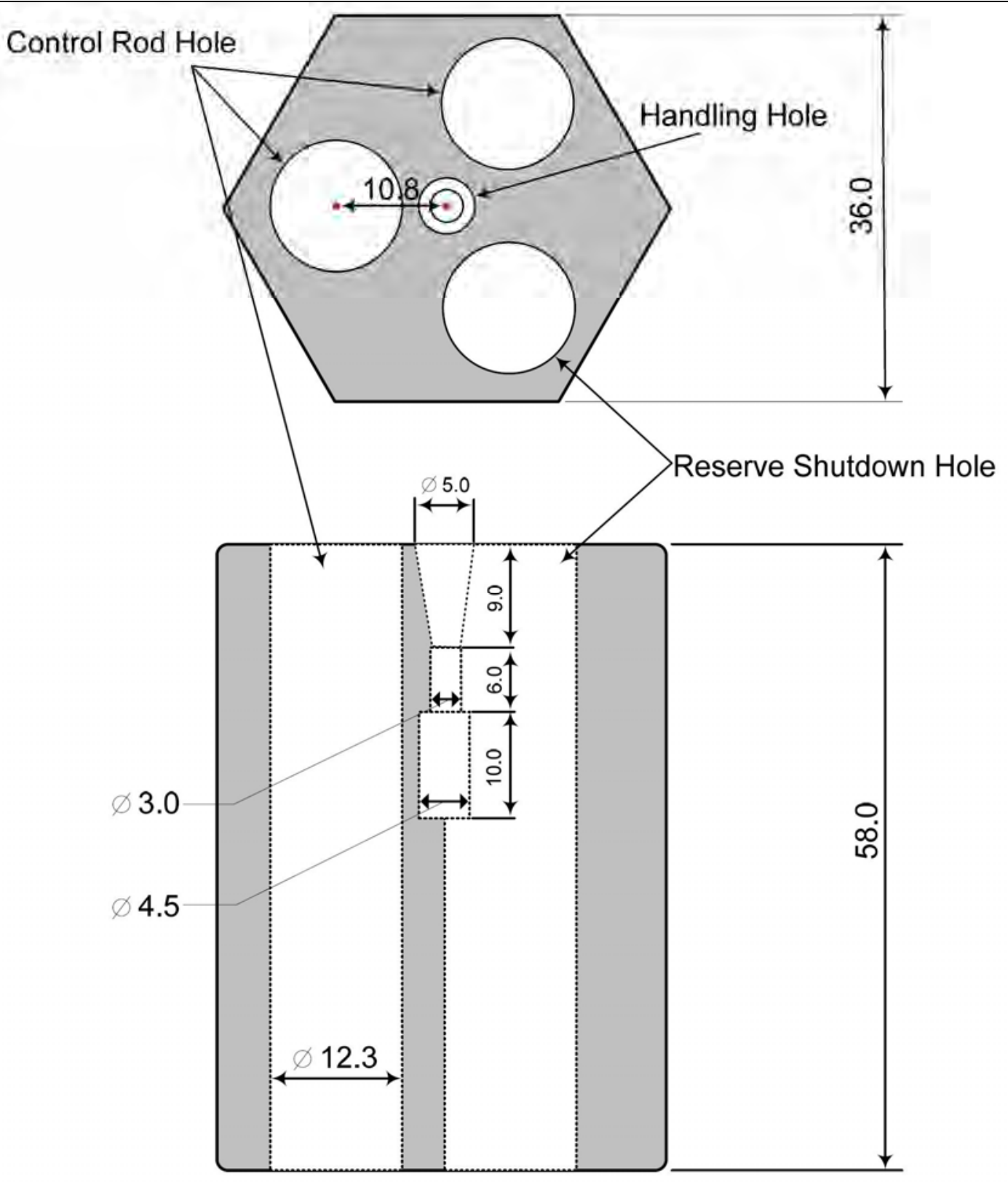

Figure 15. Control rod guide block (measurements in $\mathbf{c m}$ ). 
Utilization of MAs as a Fuel Component for Ultra-Long Life VHTR Configurations: Designs, Advantages and Limitations

Project 05-094

Final Scientific/Technical Report

\subsection{Documented Benchmark Problems for HTTR and HTR-10 Configurations}

Table 15. Benchmark Problems for HTTR Configurations

\begin{tabular}{|c|c|c|}
\hline Benchmark & Description & Benchmark Data Type \\
\hline $\begin{array}{l}\text { Critical Control Rod } \\
\text { Position } \\
(300 \mathrm{~K})\end{array}$ & 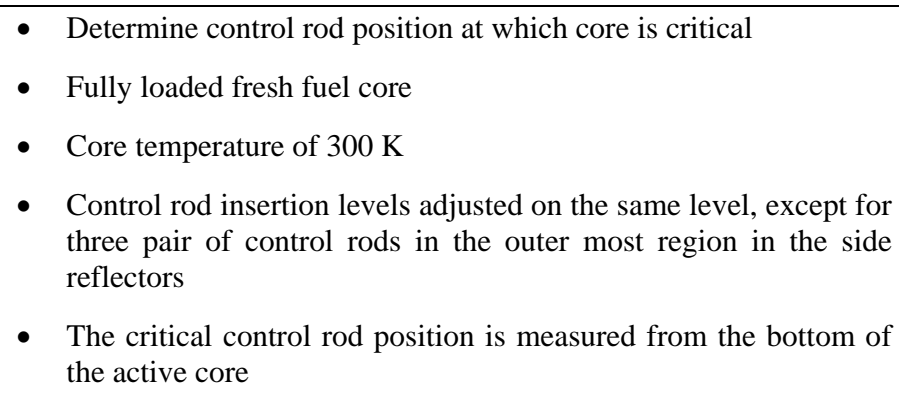 & $\begin{array}{ll} & \text { Code-to-code } \\
\text { - } & \text { Experiment-to-code }\end{array}$ \\
\hline $\begin{array}{l}\text { Critical Control Rod } \\
\text { Position } \\
(418 \mathrm{~K} \text { and } 480 \mathrm{~K})\end{array}$ & $\begin{array}{l}\text { - } \text { Determine control rod position at which core is critical } \\
\text { - } \text { Fully loaded fresh fuel core } \\
\text { - Core temperatures of } 418 \mathrm{~K} \text { and } 480 \mathrm{~K} \text {. } \\
\text { - Control rod insertion levels adjusted on the same level, except for } \\
\text { three pair of control rods in the outer most region in the side } \\
\text { reflectors } \\
\text { - The critical control rod position is measured from the bottom of } \\
\text { the active core }\end{array}$ & $\begin{array}{ll}- & \text { Code-to-code }(480 \mathrm{~K}) \\
-\quad & \text { Experiment-to-code } \\
& (418 \mathrm{~K})\end{array}$ \\
\hline$k_{\text {eff }}$ & $\begin{array}{l}\text { - Determine } k_{\text {eff }} \text { for core temperatures of 300, 340, 380, 420, } 460 \\
\text { and } 480 \mathrm{~K} \\
\text { - } \quad \text { Fully loaded fresh fuel core } \\
\text { - Control rods held at critical position for } 300 \mathrm{~K} \text { core (determined } \\
\text { in the benchmark problem } 1 \text { above) }\end{array}$ & - Code-to-code \\
\hline $\begin{array}{l}k_{\text {eff }} \text { with Control } \\
\text { Rods Fully Out }\end{array}$ & $\begin{array}{l}\text { - } \text { Determine } k_{\text {eff }} \\
\text { - } \quad \text { Fully loaded fresh fuel core } \\
\text { - } \quad \text { Core temperature of } 300 \mathrm{~K} \\
\text { - } \quad \text { Control rods removed from the core }\end{array}$ & $\begin{array}{ll}\text { - } & \text { Code-to-code } \\
\text { - } & \text { Experiment-to-code }\end{array}$ \\
\hline $\begin{array}{l}k_{\text {eff }} \text { with Control } \\
\text { Rods Fully In }\end{array}$ & $\begin{array}{l}\text { - } \text { Determine } k_{\text {eff }} \\
\text { - } \text { Fully loaded fresh fuel core } \\
\text { - } \text { Core temperature of } 300 \mathrm{~K} \\
\text { - } \quad \text { Control rods fully inserted in the core }\end{array}$ & $\begin{array}{ll}\text { - } & \text { Code-to-code } \\
\text { - } & \text { Experiment-to-code }\end{array}$ \\
\hline
\end{tabular}




\section{Utilization of MAs as a Fuel Component for Ultra-Long Life VHTR Configurations: Designs, Advantages and Limitations}

KENO6-VI Parameters:

- $\mathrm{GEN}=210$ (number of generations to be run)

- $\mathrm{NPG}=1000$ (number of neutrons per generation)

- $\mathrm{NSK}=10$ (number of generations to be)

- $\quad$ FLX=yes (collect and print fluxes)

- $\quad$ FDN=yes (collect and print fission densities)

- $\quad$ PKI=yes (print input fission spectrum)

- $\quad \mathrm{FAR}=y e s$ (generate fissions and absorptions)

- GAS=yes (print fissions and absorptions)

- Remaining parameters at default
More Data Parameters

- $\quad$ IIM=50 (max number of inner iterations to be used in the XSDRNPM calculation)

- $\mathrm{ICM}=100$ (max number of inner iterations to be used for XSDRNPM)

- $\mathrm{COF}=3$ (diffusion coefficient for transverse leakage corrections in XSDRNPM, use a flux and volume weighting across all zones)

- EPS=0.0001 (overall convergence criteria for XSDRNPM, smaller value tightens convergence)

- $\quad$ PTC=0.000001 (pointwise convergence criteria for XSDRNPM, smaller value tightens convergence)

- $\operatorname{DAN}(\mathrm{xx})=$ Dancoff factor 
Utilization of MAs as a Fuel Component for Ultra-Long Life VHTR Configurations: Designs, Advantages and Limitations

\section{Table 16. Benchmark Problems for HTR-10 Configurations}

\begin{tabular}{|c|c|c|c|}
\hline Benchmark & \multicolumn{2}{|c|}{ Description } & Benchmark Data Type \\
\hline & \multicolumn{2}{|c|}{$\begin{array}{l}\text { This benchmark problem involves calculating the amount of loading } \\
\text { (given in loading height, starting from the upper surface of the cone } \\
\text { region) for the first criticality: } k_{\text {eff }}=1.0 \text { under the atmosphere of } \\
\text { helium and core temperature of } 20^{\circ} \mathrm{C} \text {, without any control rod being } \\
\text { inserted. }\end{array}$} & $\begin{array}{ll}\text { - } & \text { Code-to-code } \\
\text { - } & \text { Experiment-to-code }\end{array}$ \\
\hline $\begin{array}{l}\text { Temperature } \\
\text { Coefficient - } \\
\text { (HTR-10: B2) }\end{array}$ & \multicolumn{2}{|c|}{$\begin{array}{l}\text { Calculation of the effective multiplication factor } k_{\text {eff }} \text { of the full core } \\
\text { ( } 5 \mathrm{~m}^{3} \text { ) under helium atmosphere and core temperatures as follows: } \\
20^{\circ} \mathrm{C} \text { (B2-1), } 120^{\circ} \mathrm{C}(\mathrm{B} 2-2) \text {, and } 250^{\circ} \mathrm{C}(\mathrm{B} 2-3) \text { respectively, } \\
\text { without any control rods being inserted. }\end{array}$} & \\
\hline $\begin{array}{l}\text { Control Rod Wo } \\
\text { for Full Core - } \\
\text { (HTR-10: B3) }\end{array}$ & \multicolumn{2}{|c|}{$\begin{array}{l}\text { This problem includes calculating the reactivity worth of the ten fully } \\
\text { inserted control rods (B3-1), and of one fully inserted control rod (B3- } \\
\text { 2, the other rods are in withdrawn position) under helium atmosphere } \\
\text { and core temperature of } 20^{\circ} \mathrm{C} \text { for full core. }\end{array}$} & - $\mathrm{C}$ \\
\hline $\begin{array}{l}\text { Control Rod Worth } \\
\text { for the Initial Core - } \\
\text { (HTR-10: B4) }\end{array}$ & \multicolumn{2}{|c|}{$\begin{array}{l}\text { Calculation of the reactivity worth of the ten fully inserted control rods } \\
\text { (B4-1) under helium atmosphere and core temperature of } 20^{\circ} \mathrm{C} \text { for a } \\
\text { loading height of } 126 \mathrm{~cm} \text {, and the differential worth of one control rod } \\
\text { (B4-2, with the other rods in the withdrawn position). The differential } \\
\text { reactivity worth is proposed to be calculated when the lower end of the } \\
\text { rod is at the following axial positions: } 394.2 \mathrm{~cm} \text {, } 383.618 \mathrm{~cm} \text {, } 334.918 \\
\mathrm{~cm}, 331.318 \mathrm{~cm}, 282.618 \mathrm{~cm}, 279.018 \mathrm{~cm} \text {, and } 230.318 \mathrm{~cm} \text {; under } \\
\text { helium atmosphere and core temperature of } 20^{\circ} \mathrm{C} \text { for a loading } \\
\text { height of } 126 \mathrm{~cm} \text {. }\end{array}$} & $\mathrm{Cc}$ \\
\hline \multicolumn{2}{|c|}{ 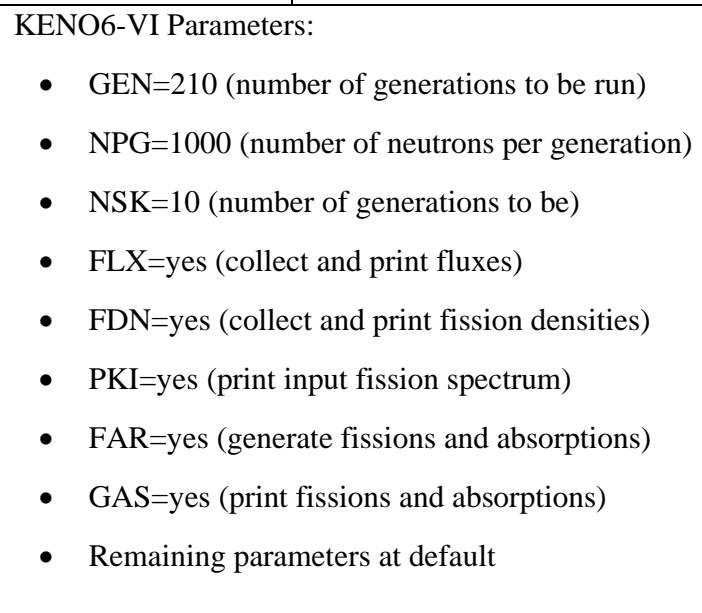 } & \multicolumn{2}{|c|}{$\begin{array}{l}\text { More Data Parameters } \\
\text { - } \quad \text { IIM=50 (max number of inner iterations to be used in the } \\
\text { XSDRNPM calculation) } \\
\text { - ICM=100 (max number of inner iterations to be used for } \\
\text { XSDRNPM) } \\
\text { - COF=3 (diffusion coefficient for transverse leakage corrections } \\
\text { in XSDRNPM, use a flux and volume weighting across all } \\
\text { zones) } \\
\text { - EPS=0.0001 (overall convergence criteria for XSDRNPM, } \\
\text { smaller value tightens convergence) } \\
\text { - PTC=0.000001 (pointwise convergence criteria for } \\
\text { XSDRNPM, smaller value tightens convergence) } \\
\text { - DAN(xx)=Dancoff factor }\end{array}$} \\
\hline
\end{tabular}




\section{Utilization of MAs as a Fuel Component for Ultra-Long Life} VHTR Configurations: Designs, Advantages and Limitations

Project 05-094

Final Scientific/Technical Report

Core temperature is defined as the temperature of the balls and all the surrounding structures included in the core physics model as described above in the HTR-10 reactor model and core configuration. Full core volume of $5 \mathrm{~m}^{3}$ is defined as the total volume of the mixed balls and the graphite balls in the cone region. Loading height is the height of the mixed balls starting from the upper surface of the cone region. 
Utilization of MAs as a Fuel Component for Ultra-Long Life

VHTR Configurations: Designs, Advantages and Limitations

Project 05-094

Final Scientific/Technical Report

\section{APPENDIX C. Selected Actinide Data}

Table 17. Actinides [ENDF/B-6.8]

\begin{tabular}{|c|c|c|}
\hline Element & Nuclide & Half-life \\
\hline \multirow[t]{4}{*}{$\mathrm{U}$} & ${ }^{234} \mathrm{U}$ & $2.457 \mathrm{E}+5$ years \\
\hline & ${ }^{235} \mathrm{U}$ & $7.037 \mathrm{E}+8$ years \\
\hline & ${ }^{236} \mathrm{U}$ & $2.342 \mathrm{E}+7$ years \\
\hline & ${ }^{238} \mathrm{U}$ & $4.468 \mathrm{E}+9$ years \\
\hline $\mathrm{Np}$ & ${ }^{237} \mathrm{~Np}$ & $2.140 \mathrm{E}+6$ years \\
\hline \multirow[t]{5}{*}{$\mathrm{Pu}$} & ${ }^{238} \mathrm{Pu}$ & 87.70 years \\
\hline & ${ }^{239} \mathrm{Pu}$ & $2.411 \mathrm{E}+4$ years \\
\hline & ${ }^{240} \mathrm{Pu}$ & $6.563 \mathrm{E}+3$ years \\
\hline & ${ }^{241} \mathrm{Pu}$ & $1.435 \mathrm{E}+1$ years \\
\hline & ${ }^{242} \mathrm{Pu}$ & $3.735 \mathrm{E}+5$ years \\
\hline \multirow[t]{4}{*}{$\mathrm{Am}$} & ${ }^{241} \mathrm{Am}$ & 432.7 years \\
\hline & ${ }^{242} \mathrm{Am}$ & 16.02 hours \\
\hline & ${ }^{242 \mathrm{~m}} \mathrm{Am}$ & 141.0 years \\
\hline & ${ }^{243} \mathrm{Am}$ & $7.370 \mathrm{E}+3$ years \\
\hline \multirow[t]{7}{*}{$\mathrm{Cm}$} & ${ }^{242} \mathrm{Cm}$ & 162 days \\
\hline & ${ }^{243} \mathrm{Cm}$ & 28.50 years \\
\hline & ${ }^{244} \mathrm{Cm}$ & 18.1 years \\
\hline & ${ }^{245} \mathrm{Cm}$ & 8.500E+3 years \\
\hline & ${ }^{246} \mathrm{Cm}$ & $4.730 \mathrm{E}+3$ years \\
\hline & ${ }^{247} \mathrm{Cm}$ & $1.600 \mathrm{E}+7$ years \\
\hline & ${ }^{248} \mathrm{Cm}$ & $3.400 \mathrm{E}+5$ years \\
\hline $\mathrm{Bk}$ & ${ }^{249} \mathrm{Bk}$ & $320 \mathrm{~d}$ \\
\hline \multirow[t]{4}{*}{$\mathrm{Cf}$} & ${ }^{249} \mathrm{Cf}$ & 350.601 years \\
\hline & ${ }^{250} \mathrm{Cf}$ & 13.08 years \\
\hline & ${ }^{251} \mathrm{Cf}$ & 897.999 years \\
\hline & ${ }^{252} \mathrm{Cf}$ & 2.645 years \\
\hline
\end{tabular}


Utilization of MAs as a Fuel Component for Ultra-Long Life VHTR Configurations: Designs, Advantages and Limitations

Final Scientific/Technical Report

\section{Table 18. Natural Uranium}

\begin{tabular}{|l|c|c|}
\hline \multirow{2}{*}{ Nuclide } & \multicolumn{2}{|c|}{ Composition } \\
\cline { 2 - 3 } & weight \% & atom \% \\
\hline${ }^{234} \mathrm{U}$ & 0.005 & 0.005 \\
\hline${ }^{235} \mathrm{U}$ & 0.711 & 0.720 \\
\hline${ }^{238} \mathrm{U}$ & 99.284 & 99.275 \\
\hline & 100.000 & 100.000 \\
\hline
\end{tabular}

Table 19. Average PWR Spent Fuel

Burnup = 41,200 MWd/MTHM, enrichment = 3.75 percent, decay time = 23 years .

(a) Reactor Grade Plutonium (RGPu) Vector

\begin{tabular}{|l|c|c|}
\hline Nuclide & Decay Heat (W/g) & Composition (atom \%) \\
\hline${ }^{238} \mathrm{Pu}$ & 0.56000 & 2.360 \\
\hline${ }^{239} \mathrm{Pu}$ & 0.00200 & 61.453 \\
\hline${ }^{240} \mathrm{Pu}$ & 0.00700 & 26.022 \\
\hline${ }^{241} \mathrm{Pu}$ & 0.00400 & 4.877 \\
\hline${ }^{242} \mathrm{Pu}$ & 0.00010 & 5.289 \\
\hline & & 100.000 \\
\hline
\end{tabular}

(b) TRU Vector

\begin{tabular}{|l|c|c|c|}
\hline Element & Nuclide & Decay Heat (W/g) & TRU Composition (atom \%) \\
\hline $\mathrm{Np}$ & ${ }^{237} \mathrm{~Np}$ & 0.00002 & 6.121 \\
\hline \multirow{5}{*}{$\mathrm{Pu}$} & ${ }^{238} \mathrm{Pu}$ & 0.56000 & 1.986 \\
\cline { 2 - 4 } & ${ }^{239} \mathrm{Pu}$ & 0.00200 & 51.718 \\
\cline { 2 - 4 } & ${ }^{240} \mathrm{Pu}$ & 0.00700 & 21.899 \\
\cline { 2 - 4 } & ${ }^{241} \mathrm{Pu}$ & 0.00400 & 4.104 \\
\cline { 2 - 4 } & ${ }^{242} \mathrm{Pu}$ & 0.00010 & 4.451 \\
\hline \multirow{3}{*}{$\mathrm{Am}$} & ${ }^{241} \mathrm{Am}$ & 0.11000 & 8.250 \\
\cline { 2 - 4 } & ${ }^{242 \mathrm{~m}} \mathrm{Am}$ & - & 0.020 \\
\cline { 2 - 4 } & ${ }^{243} \mathrm{Am}$ & 0.00700 & 1.230 \\
\hline
\end{tabular}


Utilization of MAs as a Fuel Component for Ultra-Long Life

VHTR Configurations: Designs, Advantages and Limitations

Project 05-094

Final Scientific/Technical Report

\begin{tabular}{|l|c|c|c|}
\hline \multirow{3}{*}{$\mathrm{Cm}$} & ${ }^{243} \mathrm{Cm}$ & 1.70000 & 0.003 \\
\cline { 2 - 4 } & ${ }^{244} \mathrm{Cm}$ & 2.80000 & 0.194 \\
\cline { 2 - 4 } & ${ }^{245} \mathrm{Cm}$ & - & 0.021 \\
\cline { 2 - 4 } & ${ }^{246} \mathrm{Cm}$ & - & 0.003 \\
\hline
\end{tabular}

(c) MA Vector

\begin{tabular}{|l|c|c|c|}
\hline Element & Nuclide & Decay Heat (W/g) & MA Composition (atom \%) \\
\hline $\mathrm{Np}$ & ${ }^{237} \mathrm{~Np}$ & 0.00002 & 38.635 \\
\hline \multirow{3}{*}{$\mathrm{Am}$} & ${ }^{241} \mathrm{Am}$ & 0.11000 & 52.079 \\
\cline { 2 - 4 } & ${ }^{242 \mathrm{~m}} \mathrm{Am}$ & - & 0.127 \\
\cline { 2 - 4 } & ${ }^{243} \mathrm{Am}$ & 0.00700 & 7.762 \\
\hline $\mathrm{Cm}$ & ${ }^{243} \mathrm{Cm}$ & 1.70000 & 0.021 \\
\cline { 2 - 4 } & ${ }^{244} \mathrm{Cm}$ & 2.80000 & 1.225 \\
\cline { 2 - 4 } & ${ }^{245} \mathrm{Cm}$ & - & 0.134 \\
\cline { 2 - 4 } & ${ }^{246} \mathrm{Cm}$ & - & 0.017 \\
\hline
\end{tabular}


Utilization of MAs as a Fuel Component for Ultra-Long Life

VHTR Configurations: Designs, Advantages and Limitations

Project 05-094

Final Scientific/Technical Report

\section{APPENDIX D. Project Participants and Resources}

\subsection{Contact List}

Table 20. Contact List

\begin{tabular}{|l|l|l|l|}
\hline \multicolumn{2}{|c|}{ Name } & \multicolumn{1}{c|}{ Position } & \multicolumn{1}{c|}{ E-mail } \\
\hline 1. & Pavel V. Tsvetkov & Assistant Professor & Tsvetkov@tamu.edu \\
\hline
\end{tabular}




\subsection{Resources}

This project uses existing computational capabilities of the Advanced Energy Technologies Research Group (Dr. Pavel V. Tsvetkov) at the Department of Nuclear Engineering and the Department of Nuclear Engineering Common Network:

- The Department of Nuclear Engineering computer facilities have recently been renovated and currently consist of several UNIX workstations, including computational servers and applications servers, computer lab with 30 modern PC stations; UNIX and Linux clusters for computational jobs, and etc.

- To complete tasks of the project, the Advanced Energy Technologies Research Group used five Windows workstations (two single-CPU $3.8 \mathrm{GHz} / 4 \mathrm{~GB}$ RAM and three dual CPU 3.8 GHz/4GB RAM).

The applied engineering software library of the project consists of the following code systems and software packages:

- SCALE code system (Oak Ridge National Laboratory),

- MCNP code system and auxiliary codes (Los Alamos National Laboratory),

- Dancoff-MC code system and modifications (RSICC),

- Matlab engineering software (The MathWorks, Inc.),

- HeatWave code system (Field Precision, Inc.),

- UCODE code system (U.S. Geological Survey),

- Various auxiliary software packages and codes. 


\section{Utilization of MAs as a Fuel Component for Ultra-Long Life}

VHTR Configurations: Designs, Advantages and Limitations

Project 05-094

Final Scientific/Technical Report

\section{REPORT DISTRIBUTION LIST}

Texas A\&M University:

- Project PI: P. V. Tsvetkov, tsvetkov@tamu.edu

- Participating students

- Department of Nuclear Engineering

Texas Engineering Experiment Station (TEES):

- D. Duffee, dduffee@tamu.edu

- K. Anderson, katinaanderson@tamu.edu

- Crystal Chovanec, crystalchovanec@tees.tamus.edu

- TEESdeliverables@tamu.edu

Department of Energy (DOE):

- psdrept@id.doe.gov

- M. Demott, maria.demott@nuclear.energy.gov

- K. Osborne, kenny.osborne@nuclear.energy.gov

- M. Feltus, madeline.feltus@hq.doe.gov

- C. D. Savage, buzz.savage@nuclear.energy.gov 